Université de Montréal

\title{
High-amylose carboxymethyl starch matrices for oral sustained drug-release: in vitro and in vivo evaluation
}

\author{
par \\ Maria Teresa Domingues Nabais
}

Technologie Pharmaceutique

Faculté de Pharmacie

Thèse présentée à la Faculté de Pharmacie

en vue de l'obtention du grade de $\mathrm{PhD}$

en Sciences Pharmaceutiques

option Technologie Pharmaceutique

Août 2013

(C) Maria Teresa Domingues Nabais, 2013 


\section{Université de Montréal \\ Faculté des études supérieures et postdoctorales}

Cette thèse intitulée :

High-amylose carboxymethyl starch matrices for oral sustained drug-release: in vitro and in vivo evaluation

Présentée par :

Maria Teresa Domingues Nabais

a été évaluée par un jury composé des personnes suivantes :

Prof. Jeanne Leblond Chain, président-rapporteur

Prof. Grégoire Leclair, directeur de recherche

Prof. Xavier Banquy, membre du jury

Prof. Mircea-Alexandru Mateescu, examinateur externe 


\section{Abstract}

Unmodified and modified starches represent a particularly interesting group of biodegradable and abundant excipients. They have been widely used as excipients for various purposes in tablet formulations, such as binders and/or disintegrants. Spray-dried highamylose sodium carboxymethyl starch (SD HASCA) was recently proposed as an innovating hydrophilic excipient for sustained-release (SR) in solid oral dosage forms. Amorphous highamylose sodium carboxymethyl starch (HASCA) was first produced by the etherification of high-amylose corn starch with chloroacetate. HASCA was then spray dried to obtain SD HASCA. This new excipient has shown advantageous and effective properties in the production of SR delivery systems. SR matrix tablets prepared from SD HASCA are inexpensive, simple to formulate and easy to produce by direct compression.

The main objective of the present research was to continue the development and optimization of matrix tablets using SD HASCA as the retarding excipient in view of their ultimate application as sustained drug-release delivery systems for oral administration. For this purpose, dissolution tests simulating some of the most relevant physiological conditions of the gastrointestinal tract, taking into account the nature of the polymer under investigation, were employed to evaluate the drug-release characteristics and demonstrate the performance of SD HASCA SR formulations. An exploratory clinical study was also carried out to evaluate the SR properties of this new drug delivery system in the gastrointestinal tract.

The first article presented in this thesis evaluated the drug-release characteristics and the physical integrity of formulations containing a compressed blend of drug, sodium chloride and SD HASCA in biorelevant media. The influence of different acidic $\mathrm{pH}$ values and residence times was investigated. The SR profile from an optimized SD HASCA formulation was not significantly affected by both the acidic $\mathrm{pH}$ value and the residence time in the acidic medium. These results suggest a limited influence of intra- and inter-subject variability of gastric $\mathrm{pH}$ on the release kinetics from SD HASCA matrices. In addition, the optimized formulation maintained its integrity throughout the duration of the dissolution tests. The exploratory in vivo study demonstrated extended drug absorption after oral administration of SD HASCA matrix tablets and that the matrix tablets did not disintegrate while passing through the stomach and resisted hydrolysis by $\alpha$-amylase in the intestine. 
The second article reports the development of once-daily and twice-daily SD HASCA tablets containing tramadol hydrochloride $(100 \mathrm{mg}$ and $200 \mathrm{mg})$. These SR formulations presented high crushing strengths without requiring the addition of binders, which facilitates tablet processing and handling. The compression force (CF) applied to produce the tablets did not significantly affect the drug-release profiles. The total release time from SD HASCA tablets increased significantly in function of the tablet weight and can be used to modulate the total release time from theses formulations. When exposed to a $\mathrm{pH}$ gradient and to a $40 \%$ ethanol medium, a very rigid gel formed progressively on the surface of the tablets providing controlled drug-release properties. These properties indicated that SD HASCA is a robust excipient for oral, sustained drug-release, likely to minimize the possibility of dose dumping and consequent adverse effects, even when co-administered with high doses of alcohol.

The third article investigated the effect of $\alpha$-amylase on drug-release from previously developed SD HASCA tablets containing acetaminophen and tramadol hydrochloride (Acetaminophen SR and Tramadol SR). Mathematical modeling showed that an increase in $\alpha-$ amylase concentration resulted in an increase of polymer erosion over drug diffusion as the main mechanism controlling drug-release, for both formulations and both residence times in acidic medium. However, even if the mechanism of release was affected, $\alpha$-amylase concentrations ranging from $0 \mathrm{IU} / \mathrm{L}$ to $20000 \mathrm{IU} / \mathrm{L}$ did not significantly affect the drug-release profiles from SD HASCA SR tablets, regardless of the residence time in acidic medium, the drug used, the polymer content and the different composition of each formulation.

The work presented in this thesis clearly demonstrates the value of SD HASCA as an efficient SR excipient.

Keywords: oral drug delivery; sustained-release; hydrophilic excipient; modified starch; highamylose; matrix tablet; in vitro; in vivo; $\alpha$-amylase. 


\section{Résumé}

Les amidons non modifiées et modifiés représentent un groupe d'excipients biodégradables et abondants particulièrement intéressant. Ils ont été largement utilisés en tant qu'excipients à des fins diverses dans des formulations de comprimés, tels que liants et/ou agents de délitement. Le carboxyméthylamidon sodique à haute teneur en amylose atomisé (SD HASCA) a été récemment proposé comme un excipient hydrophile à libération prolongée innovant dans les formes posologiques orales solides. Le carboxyméthylamidon sodique à haute teneur en amylose amorphe (HASCA) a d'abord été produit par l'éthérification de l'amidon de maïs à haute teneur en amylose avec le chloroacétate. HASCA a été par la suite séché par atomisation pour obtenir le SD HASCA. Ce nouvel excipient a montré des propriétés présentant certains avantages dans la production de formes galéniques à libération prolongée. Les comprimés matriciels produits à partir de SD HASCA sont peu coûteux, simples à formuler et faciles à produire par compression directe.

Le principal objectif de cette recherche était de poursuivre le développement et l'optimisation des comprimés matriciels utilisant SD HASCA comme excipient pour des formulations orales à libération prolongée. A cet effet, des tests de dissolution simulant les conditions physiologiques du tractus gastro-intestinal les plus pertinentes, en tenant compte de la nature du polymère à l'étude, ont été utilisés pour évaluer les caractéristiques à libération prolongée et démontrer la performance des formulations SD HASCA. Une étude clinique exploratoire a également été réalisée pour évaluer les propriétés de libération prolongée de cette nouvelle forme galénique dans le tractus gastro-intestinal.

Le premier article présenté dans cette thèse a évalué les propriétés de libération prolongée et l'intégrité physique de formulations contenant un mélange comprimé de principe actif, de chlorure de sodium et de SD HASCA, dans des milieux de dissolution biologiquement pertinentes. L'influence de différentes valeurs de $\mathrm{pH}$ acide et de temps de séjour dans le milieu acide a été étudiée. Le profil de libération prolongée du principe actif à partir d'une formulation de SD HASCA optimisée n'a pas été significativement affecté ni par la valeur de $\mathrm{pH}$ acide ni par le temps de séjour dans le milieu acide. Ces résultats suggèrent une influence limitée de la variabilité intra et interindividuelle du pH gastrique sur la cinétique de libération à partir de matrices de SD HASCA. De plus, la formulation optimisée a gardé 
son intégrité pendant toute la durée des tests de dissolution. L'étude in vivo exploratoire a démontré une absorption prolongée du principe actif après administration orale des comprimés matriciels de SD HASCA et a montré que les comprimés ne se sont pas désintégrés en passant par l'estomac et qu'ils ont résisté à l'hydrolyse par les $\alpha$-amylases dans l'intestin.

Le deuxième article présente le développement de comprimés SD HASCA pour une administration orale une fois par jour et deux fois par jour contenant du chlorhydrate de tramadol (100 mg et $200 \mathrm{mg})$. Ces formulations à libération prolongée ont présenté des valeurs de dureté élevées sans nécessiter l'ajout de liants, ce qui facilite la production et la manipulation des comprimés au niveau industriel. La force de compression appliquée pour produire les comprimés n'a pas d'incidence significative sur les profils de libération du principe actif. Le temps de libération totale à partir de comprimés SD HASCA a augmenté de manière significative avec le poids du comprimé et peut, de ce fait, être utilisé pour moduler le temps de libération à partir de ces formulations. Lorsque les comprimés ont été exposés à un gradient de $\mathrm{pH}$ et à un milieu à 40\% d'éthanol, un gel très rigide s'est formé progressivement sur leur surface amenant à la libération prolongée du principe actif. Ces propriétés ont indiqué que SD HASCA est un excipient robuste pour la production de formes galéniques orales à libération prolongée, pouvant réduire la probabilité d'une libération massive de principe actif et, en conséquence, des effets secondaires, même dans le cas de co-administration avec une forte dose d'alcool.

Le troisième article a étudié l'effet de $\alpha$-amylase sur la libération de principe actif à partir de comprimés SD HASCA contenant de l'acétaminophène et du chlorhydrate de tramadol qui ont été développés dans les premières étapes de cette recherche (Acetaminophen SR et Tramadol SR). La modélisation mathématique a montré qu'une augmentation de la concentration d' $\alpha$-amylase a entraîné une augmentation de l'érosion de polymère par rapport à la diffusion de principe actif comme étant le principal mécanisme contrôlant la libération de principe actif, pour les deux formulations et les deux temps de résidence en milieu acide. Cependant, même si le mécanisme de libération peut être affecté, des concentrations d' $\alpha$ amylase allant de 0 UI/L à $20000 \mathrm{UI} / \mathrm{L}$ n'ont pas eu d'incidence significative sur les profils de libération prolongée à partir de comprimés SD HASCA, indépendamment de la durée de 
séjour en milieu acide, le principe actif utilisé, la teneur en polymère et la différente composition de chaque formulation.

Le travail présenté dans cette thèse démontre clairement l'utilité de SD HASCA en tant qu'un excipient à libération prolongée efficace.

Mots-clés : administration orale de médicaments ; libération prolongée ; excipient hydrophile ; amidon modifié ; haute teneur en amylose ; comprimé matriciel ; in vitro ; in vivo ; $\alpha$-amylase. 


\section{Table of contents}

$\begin{array}{lll}\text { Abstract } & \text { i }\end{array}$

Résumé $\quad$ iii

Table of contents

List of tables $\quad$ xiii

List of figures $\quad$ XV

List of initials and abbreviations $\quad$ xxii

$\begin{array}{ll}\text { Acknowledgements } & \text { xxvi }\end{array}$

Chapter 1. Introduction 1

1.1 Pharmaceutical dosage forms intended to oral drug administration 1

1.1.1. General principles 1

1.1.2. Oral drug administration 3

1.1.3. Pharmaceutical oral dosage forms 5

1.1.4. Drug-release from oral solid dosage forms: immediate versus modified $\begin{array}{ll}\text { drug-release } & 7\end{array}$

1.1.4.1 Immediate drug-release dosage forms 7

1.1.4.2 Modified drug-release dosage forms 10

1.2 Solid dosage forms for oral sustained drug-release 15

$\begin{array}{lll}\text { 1.2.1 General principles } & 15\end{array}$

1.2.2 Multiple-unit dosage forms for sustained drug-release 15

1.2.3 Monolithic dosage forms for sustained drug-release $\quad 17$

$\begin{array}{lll}\text { 1.2.3.1 Reservoir systems } & 17\end{array}$

$\begin{array}{ll}\text { 1.2.3.2 Matrix systems } & 20\end{array}$

$\begin{array}{lll}\text { 1.2.3.3 Osmotic pumps } & 27\end{array}$

1.2.3.4 Ion exchange systems

1.2.4 The special case of hydrophilic matrix systems 30

1.2.4.1 Drug-release mechanisms from hydrophilic matrix systems 30 
1.2.4.2 Mathematical analysis of drug-release from hydrophilic matrix systems 36

1.2.5 Excipients used in hydrophilic matrix systems 40

1.2.5.1 Polymers for sustained drug-release systems $\quad 40$

1.2.5.2 Other excipients in the formulation of matrix tablets 42

1.3 Starch and starch derivatives for sustained drug-release 44

$\begin{array}{lll}\text { 1.3.1 Starch } & 44\end{array}$

1.3.1.1 Starch constituents and chemical structure $\quad 44$

1.3.1.2 Starch crystallinity and polymorphic types 47

1.3.1.3 Amylose as simple helices $\quad 48$

1.3.1.4 Starch behaviour in aqueous solution $\quad 49$

1.3.1.5 Starch applications $\quad 49$

1.3.1.6 Modified starches $\quad 50$

1.3.2 Cross-linked amylose $\quad 52$

1.3.3 Substituted amylose as a sustained drug-release agent in matrix systems $\quad 54$

1.3.3.1 Synthesis at laboratory scale and general characteristics 54

1.3.3.2 Performance of amylose substituted by 1,2-epoxypropanol (SA,G-2.7) 57

1.3.4 High-amylose sodium carboxymethyl starch 61

1.3.4.1 High-amylose sodium carboxymethyl starch produced at laboratory scale 62

1.3.4.2 High-amylose sodium carboxymethyl starch produced at pilot scale $\quad 63$

1.3.4.3 Spray-dried high-amylose sodium carboxymethyl starch 65

\subsection{Physiological factors influencing oral drug-absorption 66}

1.4.1 Physiology of the gastrointestinal tract 66

$\begin{array}{lll}\text { 1.4.1.1 Oral cavity } & 69\end{array}$

$\begin{array}{lll}\text { 1.4.1.2 Oesophagus } & 70\end{array}$

$\begin{array}{lll}\text { 1.4.1.3 Stomach } & 71\end{array}$

$\begin{array}{lll}\text { 1.4.1.4 Small intestine } & 72\end{array}$

1.4.1.5 Large intestine $\quad 75$

1.4.2 Gastrointestinal transit of pharmaceutical dosage form 76

1.4.2.1 Introduction $\quad 76$

1.4.2.2 Oesophagus 76

$\begin{array}{lll}\text { 1.4.2.3 Stomach and gastric retention and emptying } & 77\end{array}$ 
$\begin{array}{lll}\text { 1.4.2.4 Small intestine } & 80\end{array}$

1.4.2.5 Large intestine $\quad 81$

1.4.3 Barriers to drug absorption $\quad 82$

1.4.3.1 The environment within the lumen $\quad 83$

$\begin{array}{lll}\text { 1.4.3.2 } & \text { Mucus } & 83\end{array}$

1.4.3.3 Gastrointestinal membrane and drug transport mechanism $\quad 84$

$\begin{array}{lll}\text { 1.4.3.4 First-pass metabolism } & 88\end{array}$

1.5 Considerations in the selection of drug candidates for incorporation in sustained drug-release dosage forms $\quad 92$

1.5.1 Physicochemical properties of drugs influencing their viability as candidates for sustained drug-release dosage forms 92

1.5.1.1 Aqueous solubility and dissolution rate 92

1.5.1.2 Partition coefficient and molecular size 95

1.5.1.3 Biopharmaceutical Classification System 97

1.5.1.4 $p K_{a}$ (logarithmic measure of the acid dissociation constant) 98

1.5.1.5 Drug stability 98

1.5.2 Biological and pharmacological properties of drugs influencing their viability as candidates for sustained drug-release dosage forms 100

1.5.2.1 Size of dose, biological half-life and duration of action $\quad 100$

$\begin{array}{ll}\text { 1.5.2.2 Absorption properties } & 101\end{array}$

$\begin{array}{lll}\text { 1.5.2.3 Distribution } & 102\end{array}$

$\begin{array}{lll}\text { 1.5.2.4 Metabolism } & 103\end{array}$

1.5.2.5 Safety margin and side effects of the drug 104

1.5.2.6 Therapeutic goal, disease state and circadian rhythm 105

1.6 Pharmacokinetic considerations and analysis in the design of dosage forms 106

1.6.1 Importance of biopharmaceutics, pharmacokinetics and pharmacodynamics 106

1.6.2 Experimental approaches of pharmacokinetics 108

$\begin{array}{lll}\text { 1.6.2.1 Measurement of drug concentrations } & 108\end{array}$

1.6.2.2 Plasma drug concentrations as a function of time 109

1.6.3 Theoretical aspects of pharmacokinetics 113

1.6.3.1 Development of pharmacokinetic models 113 
1.6.3.2 Compartmental pharmacokinetic models 114

$\begin{array}{lll}\text { 1.6.3.3 Pharmacokinetic parameters } & 116\end{array}$

1.6.3.4 Estimation of the cumulative relative fraction of drug absorbed after oral absorption

$\begin{array}{lll}\text { 1.6.3.5 Statistics in pharmacokinetic studies } & 120\end{array}$

\subsection{In vitro-in vivo correlations}

1.7.1 Importance of in vitro-in vivo correlations in the development and optimization of dosage forms

1.7.2 Correlation levels

1.7.3 Considerations in dissolution test method development for IVIVC

1.7.3.1 Importance of dissolution testing

1.7.3.2 Dissolution apparatus selection

1.7.3.3 Dissolution test media and methods

1.7.3.4 Statistical comparison of dissolution profiles

$\begin{array}{llr}2.1 & \text { General objective } & 160\end{array}$

$\begin{array}{lll}2.2 & \text { Specific objectives } & 160\end{array}$

\section{Chapter 3. High-amylose starch matrices for oral sustained drug-release:} In vitro and in vivo evaluation 163

3.1 Presentation of the article and contribution of the authors 164

$\begin{array}{lll}3.2 & \text { Abstract } & 165\end{array}$

$\begin{array}{lll}3.3 & \text { Introduction } & 165\end{array}$

3.4 Material and methods $\quad 167$

$\begin{array}{lll}3.4 .1 & \text { Materials } & 167\end{array}$

$\begin{array}{lll}\text { 3.4.2 Preparation of HASCA tablets } & 167\end{array}$ 
$\begin{array}{lll}\text { 3.4.3 Drug-release studies } & 168\end{array}$

3.4.4 Pharmacokinetics in healthy human volunteers 169

3.4.5 High-performance liquid chromatography (HPLC) analysis of plasma samples 170

3.4.6 Pharmacokinetic data analysis $\quad 171$

$\begin{array}{lll}3.4 .7 & \text { IVIVC } & 172\end{array}$

3.5 Results and discussion 172

3.5.1 In vitro drug-release characteristics from HASCA matrix tablets 172

3.5.2 Pharmacokinetics in healthy human volunteers 176

$\begin{array}{lll}3.5 .3 & \text { IVIVC } & 180\end{array}$

$\begin{array}{lll}3.6 & \text { Conclusion } & 182\end{array}$

$\begin{array}{lll}3.7 & \text { Acknowledgements } & 182\end{array}$

$\begin{array}{llr}3.8 & \text { References } & 182\end{array}$

Chapter 4. High-amylose carboxymethyl starch matrices: development and characterization of tramadol hydrochloride sustained-release tablets for $\begin{array}{ll}\text { oral administration } & 185\end{array}$

4.1 Presentation of the article and contribution of the authors 186

$\begin{array}{llr}4.2 & \text { Abstract } & 186\end{array}$

$\begin{array}{llr}4.3 & \text { Introduction } & 187\end{array}$

4.4 Materials and methods $\quad 190$

4.4.1 Materials 190

4.4.2 Preparation of HASCA tablets 190

4.4.3 Tablets characterization and in vitro drug-release evaluation 192

4.4.4 Modifications to the United States Pharmacopeia (USP) method 194

4.4.5 Statistical analysis and formulations comparison 195

$\begin{array}{lll}4.5 & \text { Results and discussion } & 195\end{array}$

4.5.1 Measurement of tablet crushing strengths in order to guarantee the reproducibility of the spray-drying method 195

4.5.2 Formulation screening and development of twice-daily and once-daily SD HASCA formulations with $100 \mathrm{mg}$ and $200 \mathrm{mg}$ of tramadol hydrochloride 196 
4.5.3 Decrease of the tablet surface area in order to increase the in vitro release time of tramadol hydrochloride from twice-daily and once-daily SD HASCA matrix tablets

4.5.3.1 Preliminary crushing strengths measurements

4.5.3.2 Influence of CF on tramadol hydrochloride release from SD HASCA tablets

4.5.3.3 In vitro release of tramadol hydrochloride from optimal twice-daily and once-daily SD HASCA matrix tablets

4.5.4 In vitro release of tramadol hydrochloride from SD HASCA tablets under ethanolic conditions

\subsection{Conclusions}

4.7 Acknowledgements

\subsection{References}

\section{Chapter 5. Spray-dried high-amylose sodium carboxymethyl starch: impact of $\alpha$-amylase on drug-release profile}

5.1 Presentation of the article and contribution of the authors

\subsection{Abstract}

\subsection{Introduction}

\subsection{Materials and methods 223}

$\begin{array}{lll}5.4 .1 & \text { Materials } & 223\end{array}$

5.4.2 Preparation of SD HASCA formulations 224

$\begin{array}{lll}\text { 5.4.3 Drug-release evaluation } & 224\end{array}$

$\begin{array}{lll}\text { 5.4.3.1 Dissolution studies } & 224\end{array}$

5.4.3.2 Statistical analysis and release profiles comparison 225

5.4.3.3 Drug-release mechanism characterization 226

\subsection{Results and discussion

5.5.1 Influence of $\alpha$-amylase and of the residence time in SGF on the drug-release $\begin{array}{ll}\text { profiles from SD HASCA tablets } & 227\end{array}$

5.5.1.1 $\alpha$-Amylase does not significantly affect the drug-release profiles $\quad 227$ 
5.5.1.2 Residence time in SGF does not significantly affect the drug-release profiles

5.5.2 Contribution of $\alpha$-amylase-catalyzed erosion to the overall drug-release mechanism

\subsection{Conclusions}

5.7 Acknowledgments

5.8 References

Chapter 6. General discussion, conclusions and perspectives

\subsection{General discussion}

6.1.1 Advantages of SD HASCA

6.1.2 Influence of $\mathrm{pH}$, gastric residence time, $\alpha$-amylase and an alcoholic medium on the drug-release from SD HASCA matrices

6.1.3 Factors influencing the gel layer formation and drug-release

6.1.4 In vivo studies and establishment of a Level A IVIVC

6.1.5 Mechanism controlling drug-release from SD HASCA matrices: Fickian diffusion versus relaxation/erosion

\subsection{Conclusions and perspectives}

6.2.1 Characterization of the gel layer formed by SD HASCA

6.2.2 In vivo studies and the establishment of a Level A IVIVC

6.2.3 Industrial feasibility of spray drying and the compressive methods used in this study

6.2.3.1 Spray drying

6.2.3.2 Tablet compressing machines 


\section{List of tables}

Table 1.1 Diffusional exponent for drug-release $(n)$ and associated drug-release mechanism from polymeric sustained delivery systems. Table reproduced from (Peppas and Sahlin 1989) with permission of Elsevier Ltd.

Table 1.2 Examples of substances used as excipients in tablet formulation (Alderborn 2002; Kottke and Rudnic 2002).

Table 1.3 Biological and physical parameters of the human gastrointestinal tract (approximate values) (Daugherty and Mrsny 1999; Ashford 2002).

Table 1.4 Levels of approximate solubilities.

Table 1.5 Biopharmaceutical Classification System (BCS).

Table $3.1 \mathrm{pH}$ gradient conditions used to simulate various possibilities of gastric $\mathrm{pHs}$ and residence times encountered by HASCA matrices during their gastrointestinal transit.

Table 3.2 Pharmacokinetic parameters of acetaminophen (mean \pm SD and $\% \mathrm{CV}$ ) following oral administration of the HASCA SR formulation and the IR tablet.

178

Table 4.1 SD HASCA tablets formulation, press used, target CF and thickness and selected twice-daily and once-daily tablets.

Table 4.2 Time to release 50\% (T50\%) and 90\% (T90\%) of drug from SD HASCA tablets with total weights between $400 \mathrm{mg}$ and $800 \mathrm{mg}$, and \% of drug (w/w) between $12.5 \%$ and $60 \%$.

Table 4.3 Time to release $50 \%$ and $90 \%$ of tramadol hydrochloride [T50\%, T90\%] from twice-daily (every 12 hours) and once-daily (every 24 hours) commercially SR formulations and SD HASCA formulations (produced with the SSP), with $100 \mathrm{mg}$ and $200 \mathrm{mg}$ of drug. Release-times are presented in hours.

Table 4.4 Time to release $50 \%$ and $90 \%$ of tramadol hydrochloride [T50\%, T90\%] for SD HASCA tablets compressed at different CF between 1 and 2.5 tons $/ \mathrm{cm}^{2}$ and similarity factors $\left(f_{2}\right)$ between tablets weighting 700-mg tablets with $100 \mathrm{mg}$ of tramadol hydrochloride, compressed with a 30 -tons manual HP at different CF.

205

Table 4.5 Time to release $50 \%$ and $90 \%$ (hours) of tramadol hydrochloride [T50\%, T90\%] from twice-daily and once-daily SD HASCA tablets with $100 \mathrm{mg}$ and $200 \mathrm{mg}$ of drug, 
compressed using a 30-tons manual hydraulic press at 2.5 tons $/ \mathrm{cm}^{2}$ (HP) or a singlestroke press (SSP).

Table 5.1 Similarity factors $\left(f_{2}\right)$ between the release profiles from Acetaminophen SR and Tramadol SR at different $\alpha$-amylase concentrations.

Table 5.2 Similarity factors $\left(f_{2}\right)$ between the release profiles from Acetaminophen SR and Tramadol SR at different residence times in simulated gastric fluid (pH 1.2). 235

Table 5.3 Values of $2 a / l$, Aspect Ratio (where $2 a$ is the diameter and $l$ is the thickness of the tablet); $m$, the estimated Fickian diffusion exponent; $k_{2} / k_{1}$, the erosion and diffusion kinetic constants ratio) and $R^{2}$, the correlation coefficient, for Acetaminophen SR and Tramadol SR, the two residence times in the acidic medium and the three $\alpha$-amylase concentrations tested.

Table 5.4 Time at which R/F became equal or superior to 1 , for the residence times in the simulated gastric fluid ( $\mathrm{pH} 1.2$ ) and the $\alpha$-amylase concentrations tested. 


\section{List of figures}

Figure 1.1 Representation of the three phases between drug administration and the attainment of the therapeutic effect: the biopharmaceutical phase, the pharmacokinetic phase, and the pharmacodynamic phase.

Figure 1.2 Difference between the plasma drug concentrations versus time profiles for immediate-release and sustained-release dosage forms.

Figure 1.3 Diffusion of drug across the polymeric membrane of a reservoir system and across an inert matrix system.

Figure 1.4 Drug-release in function of the type of matrix system. The blue represents the initial size of the matrix with drug; the yellow represents the region of the polymeric matrix with the remaining drug; the gray represents the swollen matrix; and the arrows represent the diffusing drug.

Figure 1.5 Elementary osmotic pump with a solid osmotic core, a semipermeable membrane and, in this example, a single hole drilled through the membrane. Figure reproduced from (Simó, Cifuentes et al. 2003) with permission from Elsevier Ltd.

Figure 1.6 Osmotic pump consisting of an osmotically active bilayer core enclosed in a semipermeable tablet shell membrane, which is permeable to water, but not the drug or osmotic components, and has a laser-drilled orifice on the drug-layer side of the tablet. The bilayer core is comprised of a drug layer and a push layer. Figure reproduced from (Palangio, Northfelt et al. 2002) with permission from Elsevier Ltd.

Figure 1.7 Schematic representation of drug-release from hydrophilic polymer matrix tablets.

Figure 1.8 Fronts in the swelling process (swelling, diffusion and erosion) of hydrophilic matrices during drug-release. The behaviour of the gel layer was studied using a colorimetric technique. HPMC matrices containing soluble and coloured buflomedil pyridoxal phosphate were prepared by wet granulation. Swelling studies were performed by clamping each matrix between two transparent Plexiglas ${ }^{\circledR}$ disks and the assembled device was introduced into the vessel of a USP 23 apparatus 2 containing distilled water $\left(37^{\circ} \mathrm{C}, 200 \mathrm{rpm}\right)$. At fixed times during swelling, the device was taken out of the vessel and pictures of the disc matrix base were video-recorded. The front 
distance was then measured in pixels and these were converted into length units. Figure adapted from (Colombo, Bettini et al. 1999) with permission of Elsevier Ltd.

Figure 1.9 Gel layer thickness as a function of time for HPMC matrices containing different percentages of buflomedil pyridoxal phosphate, using the same conditions of Figure 1.8. Figure reproduced from (Colombo, Bettini et al. 1999) with permission of Elsevier Ltd.

Figure 1.10 Variation of the Fickian diffusional exponent, $m$, with the aspect ratio, $2 a / l$, where $2 a$ is the diameter and $l$ is the thickness (height) of the device. Figure reproduced from (Peppas and Sahlin 1989) with permission of Elsevier Ltd.

Figure 1.11 Schematic representation of the linear amylose macromolecule with its $\alpha-(1 \rightarrow 4)-$ linked D-glucose units.

Figure 1.12 Schematic representation of the branched amylopectin macromolecule with its $\alpha$ $(1 \rightarrow 4)$-linked D-glucose backbones, interlinked by $\alpha-(1 \rightarrow 6)$-linkages.

Figure 1.13 Comparison of units cells and helix packing in A- and B-amylose. Reproduced from (Hsien-Chih and Sarko 1978) with permission of Elsevier Ltd.

Figure 1.14 General representation of the synthesis of SA polymers at laboratory scale. 55

Figure 1.15 Release rate of acetaminophen from SA,G-2.7 matrices (compressed at CF between 0.5 and 5.0 tons) as a function of dissolution time, showing a nearly constant drug-release from approximately $1 \mathrm{~h}$ to $6 \mathrm{~h}$, and three different regions for the drugrelease rates. Figure reproduced from (Moghadam, Wang et al. 2007) with permission of Elsevier Ltd.

Figure 1.16 Reaction of substitution of amylose by sodium chloroacetate.

Figure 1.17 General anatomy of gastrointestinal tract. Figure reproduced from (MacFarlane and Stover 2008) with permission of Elsevier Ltd.

Figure 1.18 Epithelium, lamina propria and villi in the jejunal mucosa. Figure reproduced from (Moghaddami, Cummins et al. 1998) with permission of Elsevier Ltd.

Figure 1.19 Schematic representation of the interdigestive motility pattern, frequency of contraction forces during each phase and average time period for each phase. Figure reproduced from (Prajapati, Jani et al. 2013) with permission of Elsevier Ltd. 
Figure 1.20 Fluid mosaic model of cell membranes (epithelial and other cell membranes) with the integral and peripheral proteins embedded in the phospholipid bilayer. Figure reproduced from (Fry 2007) with permission of Elsevier Ltd.

Figure 1.21 Passive transport (simple diffusion or diffusion via pores) and carrier-mediated transport (facilitated diffusion and active transport) across the plasma membrane. Figure reproduced from (Baynes and Riviere 2010) with permission of Elsevier Ltd.

Figure 1.22 Pathways of epithelial permeability. Transcellular permeability is associated with solute or water movement through intestinal epithelial cells. Paracellular permeability is associated with movement in the intercellular space between epithelial cells and is regulated by tight junctions (one of the components responsible for the contact between epithelial cells and for the regulation of transport between extracellular and intracellular space). Figure reproduced from (Groschwitz and Hogan 2009) with permission of Elsevier Ltd.

Figure 1.23 Main metabolic pathways of tramadol. The main compounds responsible for tramadol analgesia are marked in bold. Figure reproduced from (Leppert 2011) with permission of Karger Publishers.

Figure 1.24 Typical plasma drug concentration versus time after oral administration of a conventional dosage form, representing the maximum concentration in plasma, $C_{\max }$, the time to reach the maximum concentration, $T_{\max }$, the therapeutic range, the MTC and the MEC, the onset time and the duration of action.

Figure 1.25 Plasma concentration versus time profile of acetaminophen in one volunteer after an intravenous single dose. Figure reproduced from (Ing-Lorenzini, Desmeules et al. 2009) with permission of Elsevier Ltd.

Figure 1.26 Schematic representations of one-, two-, and three-compartment models. 116

Figure 1.27 Graphical illustration of the application of the trapezoidal rule to numerically estimate the AUC for first-order absorption data. Figure reproduced from (Byers and Sarver 2009) with permission of Elsevier Ltd.

Figure 1.28 Example of Level A IVIVC. (A) In vitro dissolution profiles of slow ( $\mathbf{\square}$ :), medium (:), and fast ( $\mathbf{\Delta}$ :) drug formulations. (B) In vivo studies provide plasma drug concentration of each formulation (gray lines), which can be converted to fractional 
absorption profile (black lines) by deconvolution. (C) Level A IVIVC can be derived from the fractional dissolution in vitro and the fractional absorption in vivo. Figure reproduced from (Lu, Kim et al. 2011) with permission of Elsevier Ltd.

126

Figure 1.29 Example of Level B (A) and Level C (B) IVIVC. Figure reproduced from (Lu, Kim et al. 2011) with permission of Elsevier Ltd.

Figure 1.30 Schematic representation of Apparatus 1 (rotating basket) and Apparatus 2 (paddle assembly) dissolution vessels.

Figure 3.1 Cumulative percent of acetaminophen released in vitro from HASCA matrices containing $40 \%$ of drug and increasing concentrations of $\mathrm{NaCl}(\checkmark: 17.5 \%$ of $\mathrm{NaCl}, \square$ : $22.5 \%$ of $\mathrm{NaCl}, \mathbf{\square}: 27.5 \%$ of $\mathrm{NaCl})$.

Figure 3.2 Cumulative percentage of acetaminophen released in vitro from optimized HASCA matrices $(32.5 \%$ of HASCA, $40 \%$ of acetaminophen, and $27.5 \%$ of $\mathrm{NaCl}$ ) in $\mathrm{pH}$ gradients with different acidic $\mathrm{pH}$ conditions $(\bullet: 10 \mathrm{~min}$ at $\mathrm{pH} 1.2, \diamond: 1 \mathrm{hr}$ at $\mathrm{pH} 1.2$, $\square: 3 \mathrm{hrs}$ at $\mathrm{pH} 3.5, \mathbf{\Delta}: 6 \mathrm{hrs}$ at $\mathrm{pH} 4.5, \mathrm{O}$ : no acidic medium).

Figure 3.3 Acetaminophen plasma concentration (expressed in $\mu \mathrm{g} / \mathrm{mL} / \mathrm{kg}$ ) vs. time profiles (mean \pm SD) following oral administration of the HASCA SR formulation ( $\square$ ) and the commercial IR tablet $(\diamond)$.

Figure 3.4 Cumulative percentage (mean $\pm \mathrm{SD}$ ) of acetaminophen absorbed following the oral administration of HASCA SR matrix tablets to 5 healthy volunteers.

Figure 3.5 IVIVC plot for HASCA SR matrix tablets administered to 5 healthy volunteers volunteer 1, O: volunteer 2, x: volunteer 3, O: volunteer 4, $\mathbf{\square}$ : volunteer 5, average, - - - -: linear).

Figure 4.1 Plastic cage used in the dissolution experiments to avoid eventual adhesion to the vessel or floating.

Figure 4.2 Cumulative \% of tramadol hydrochloride released in vitro from once-daily commercial formulations (ם: Tridural ${ }^{\circledR} 100 \mathrm{mg}, \boldsymbol{\Delta}$ : Tridural $^{\circledR} 200 \mathrm{mg}, \diamond$ : Ralivia ${ }^{\circledR}$ $200 \mathrm{mg}, \bullet:$ Zytram $^{\circledR}$ XL $\left.200 \mathrm{mg}\right)$. 200

Figure 4.3 Cumulative percentage of tramadol hydrochloride released in vitro from once-daily SD HASCA formulations compressed using a single-stroke press machine (SSP), and once-daily commercial formulations ( $\square$ : 700-mg tablets with $100 \mathrm{mg}$ of drug, $\bullet$ : 800- 
mg tablets with $200 \mathrm{mg}$ of drug, $\boldsymbol{\Delta}$ : Tridural $^{\circledR} 100 \mathrm{mg}$, and $\diamond$ : Tridural $^{\circledR} 200 \mathrm{mg}$ ). Tests were performed with the tablets placed inside a cage. $f_{2}$ values: 70.66 between 700 -mg tablets with $100 \mathrm{mg}$ and Tridural ${ }^{\circledR} 100 \mathrm{mg}, 79.80$ between 800 -mg tablets with $200 \mathrm{mg}$ and Tridural $^{\circledR} 200 \mathrm{mg}, 93.14$ between 700-mg tablets with $100 \mathrm{mg}$ and 800-mg tablets with $200 \mathrm{mg}$.

Figure 4.4 Cumulative percentage of tramadol hydrochloride released in vitro from twice-daily SD HASCA formulations, compressed with the single-stroke press machine, and twice-daily commercial formulations $(\diamond$ : 450-mg tablets with $100 \mathrm{mg}$ of drug, $\boldsymbol{\square}$ : 500-mg tablets with $200 \mathrm{mg}$ of drug, $\mathbf{\Delta}$ : Topalgic ${ }^{\circledR}$ LP $100 \mathrm{mg}$, and ๑ : Topalgic ${ }^{\circledR}$ LP $200 \mathrm{mg}$ ). Tests were performed with the tablets placed inside a cage. $f_{2}$ values: 54.52 between 450-mg tablets with $100 \mathrm{mg}$ and Topalgic ${ }^{\circledR}$ LP $100 \mathrm{mg}, 52.36$ between 500 $\mathrm{mg}$ tablets with $200 \mathrm{mg}$ of drug and Topalgic ${ }^{\circledR}$ LP $200 \mathrm{mg}$, 88.01 between 450-mg tablets with $100 \mathrm{mg}$ and 500-mg tablets with $200 \mathrm{mg}$ of drug.

Figure 4.5 Cumulative percentage of tramadol hydrochloride released in vitro from once-daily SD HASCA tablets with $100 \mathrm{mg}$ of drug and once-daily commercial formulations with $100 \mathrm{mg}$ of drug in a $\mathrm{pH}$ gradient versus a $40 \%$ ethanol hydro-alcoholic medium SD HASCA with $100 \mathrm{mg}$ in a $\mathrm{pH}$ gradient, $\diamond$ : Tridural ${ }^{\circledR} 100 \mathrm{mg}$ in a $\mathrm{pH}$ gradient, • : SD HASCA with $100 \mathrm{mg}$ in a $40 \%$ ethanolic medium $\mathbf{\square}$ : Tridural ${ }^{\circledR} 100 \mathrm{mg}$ in a $40 \%$ ethanolic medium. $f_{2}$ values: 70.66 between 700-mg tablets with $100 \mathrm{mg}$ of drug and Tridural $^{\circledR} 100 \mathrm{mg}$ in $\mathrm{pH}$ gradient, 88.51 between $700-\mathrm{mg}$ tablets with $100 \mathrm{mg}$ of drug and Tridural $^{\circledR} 100 \mathrm{mg}$ in a $40 \%$ ethanol hydro-alcoholic medium.

Figure 4.6 700-mg tablets with $100 \mathrm{mg}$ of tramadol hydrochloride in: (a) pH gradient and (b) $40 \%$ ethanol hydro-alcoholic medium (b); Tridural ${ }^{\circledR} 100 \mathrm{mg}$ in: (c) $\mathrm{pH}$ gradient and (d) $40 \%$ ethanol hydro-alcoholic medium.

Figure 5.1 (A) Effect of $\alpha$-amylase concentration on the release of acetaminophen from Acetaminophen $\mathrm{SR}$ in a simulated gastrointestinal $\mathrm{pH}$, where the residence time in simulated gastric fluid ( $\mathrm{pH}$ 1.2) was $1 \mathrm{~h}$, and the concentrations of $\alpha$-amylase in simulated intestinal fluid ( $\mathrm{pH}$ 6.8) were: 0 IU/L (dashed line), $5000 \mathrm{IU} / \mathrm{L}$ (dotted line) and $20000 \mathrm{IU} / \mathrm{L}$ (continuous line). 
Figure 5.1 (B) Effect of $\alpha$-amylase concentration on the in vitro release of acetaminophen from Acetaminophen $\mathrm{SR}$ in a simulated gastrointestinal $\mathrm{pH}$, where the residence time in simulated gastric fluid ( $\mathrm{pH} \mathrm{1.2)} \mathrm{was} 3 \mathrm{~h}$, and the concentrations of $\alpha$-amylase in simulated intestinal fluid ( $\mathrm{pH}$ 6.8) were: 0 IU/L (dashed line), 5000 IU/L (dotted line) and $20000 \mathrm{IU} / \mathrm{L}$ (continuous line).

Figure 5.2 (A) Effect of $\alpha$-amylase concentration on the in vitro release of tramadol hydrochloride from Tramadol SR in a simulated gastrointestinal $\mathrm{pH}$, where the residence time in simulated gastric fluid ( $\mathrm{pH}$ 1.2) was $1 \mathrm{~h}$, and the concentrations of $\alpha$ amylase in simulated intestinal fluid ( $\mathrm{pH}$ 6.8) were: 0 IU/L (dashed line), 5000 IU/L (dotted line) and $20000 \mathrm{IU} / \mathrm{L}$ (continuous line).

Figure 5.2 (B) Effect of $\alpha$-amylase concentration on the in vitro release of tramadol hydrochloride from Tramadol $\mathrm{SR}$ in a simulated gastrointestinal $\mathrm{pH}$, where the residence time in simulated gastric fluid ( $\mathrm{pH} 1.2)$ was $3 \mathrm{~h}$, and the concentrations of $\alpha$ amylase in simulated intestinal fluid ( $\mathrm{pH}$ 6.8) were: 0 IU/L (dashed line), $5000 \mathrm{IU} / \mathrm{L}$ (dotted line) and $20000 \mathrm{IU} / \mathrm{L}$ (continuous line).

Figure 5.3 Acetaminophen-release rate from Acetaminophen SR tablets exposed $1 \mathrm{~h}$ to

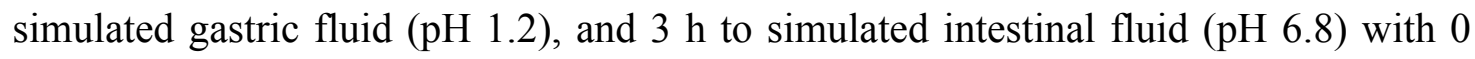
IU/L (dashed line), 5000 IU/L (dotted line) and 20000 IU/L (continuous line) of $\alpha-$ amylase. The arrows represent the time at which the tablets were placed $(1 \mathrm{~h})$ and were withdrawn (4 h) from the medium with $\alpha$-amylase.

Figure 6.1 Cumulative percent of acetaminophen released in vitro from $\square$ : SD HASCA matrices containing $40 \%$ of acetaminophen, $27.5 \%$ of $\mathrm{NaCl}$ and $32.5 \%$ of polymer; $\mathbf{\square}$ : HPMC matrices containing $40 \%$ of acetaminophen, $27.5 \%$ of $\mathrm{NaCl}$ and $32.5 \%$ of polymer; and from $\diamond$ : HPMC matrices containing $40 \%$ of acetaminophen, $0 \%$ of $\mathrm{NaCl}$ and $60 \%$ of polymer. The number of replicates was three.

Figure 6.2 600-mg SD HASCA tablet matrices (40\% acetaminophen, $27.5 \% \mathrm{NaCl}, 32.5 \% \mathrm{SD}$ HASCA) after immersion in a $\mathrm{pH}$ gradient simulation the $\mathrm{pH}$ evolution of the gastrointestinal tract $(\mathrm{pH} 1.2$ for $1 \mathrm{~h}, \mathrm{pH} 6.8$ for $3 \mathrm{~h}$, and $\mathrm{pH} 7.4$ until the end of the dissolution test): a) $16 \mathrm{~h}$ of immersion (gel layer formation), and b) $22 \mathrm{~h}$ of immersion 
(total hydration of the matrix). Figure reproduced from (Brouillet, Bataille et al. 2008) with permission of Elsevier Ltd. 


\section{List of initials and abbreviations}

ADME: absorption, distribution, metabolism and excretion

AUC: area under the plasma concentration-time curve

BCS: Biopharmaceutical Classification System

CF: compression force(s)

CL: clearance

$C_{\max }$ : maximum drug concentration in plasma

CMC: sodium carboxymethyl cellulose

CM-HAS: high-amylose sodium carboxymethyl starch with higher degree of substitution

CR: controlled-release

CYP: cytochrome P450

$\mathrm{D}_{\text {oct: }}$ octanol/water distribution coefficient at $\mathrm{pH} 7.4$

DS: degree of substitution

$\mathrm{ED}_{50}$ : minimum effective dose for $50 \%$ of the population

EMEA: European Agency for the Evaluation of Medical Products

FDA: Food and Drug Administration

FIP: International Pharmaceutical Federation

HASCA: high-amylose sodium carboxymethyl starch

$\mathrm{HCl}$ : hydrochloric acid

HEC: hydroxyethyl cellulose

HP: hydraulic press

HPC: hydroxypropyl cellulose

HPLC: high-performance liquid chromatography

HPMC: hydroxypropyl methylcellulose

IR: immediate-release

IVIVC: in vitro-in vivo correlation(s)

IVIVR: in vitro-in vivo relationship(s)

$k$ : elimination rate constant of the drug

$k_{\mathrm{a}}$ : absorption rate constant of the drug

$K_{\mathrm{a}}$ : dissociation constant 
LADME: liberation, absorption, distribution, metabolism and excretion

LC: liquid chromatography

MC: mass spectrometer

MDR1: multidrug resistance 1 gene

$\mathrm{MDT}_{\text {vitro }}$ : mean in vitro dissolution time

$\mathrm{MDT}_{\text {vivo: }}$ : mean in vivo dissolution time

MEC: minimum effective concentration

MMC: migrating motor complex

MR: modified-release

MRT: mean in vivo residence time

MTC: minimum toxic concentration

$\mathrm{NaCl}$ : sodium chloride

NMR: nuclear magnetic resonance

PD: pharmacodynamics

Pg-p: P-glycoprotein

PK: pharmacokinetics

$p K_{a}: \operatorname{logarithmic}$ measure of the acid dissociation

$\mathrm{P}_{\text {oct: }}$ octanol/water partition coefficient

SA: substituted amylose

SA,CA- $n$ : substituted amylose polymers substituted with chloroacetate

SA, G- $n$ : substituted amylose polymers substituted with glycidol

SA,R- $n$ : substituted amylose polymers, where R represents the substituent and $n$ the degree of substitution

SA/V: surface area/volume

SD: standard deviation

SD: spray-dried

SD HASCA: spray-dried high-amylose sodium carboxymethyl starch

SEM: scanning electron microscopy

SGF: simulated gastric fluid

SIF: simulated intestinal fluid

SR: sustained-release 
SSP: single-stroke press

SULT: sulfotransferase

SUPAC: scale-up and post-approval changes

$t_{1 / 2}$ : elimination half-life

$\mathrm{TD}_{50}$ : toxic dose of drug for $50 \%$ of the population

$T_{\max }$ : period of time required to achieve the maximum drug concentration in plasma

TT: tablet thickness

TW: tablet weight

UDP: uridine diphosphate

UGT: UDP-glucuronosyltransferase

USP: United States Pharmacopeia

$V_{\mathrm{d}}$ : apparent volume of distribution of a drug

XRD: X-ray diffraction 
To my parents 


\section{Acknowledgements}

First of all, I would like to express my sincere gratitude to my first director of research, Prof. Louis Cartilier, for his assistance and guidance, his confidence and his encouragement.

I would also like to thank Prof. Grégoire Leclair for having accepted to become my new director and for his guidance during the last two years, without which finishing this $\mathrm{PhD}$ would have never been possible.

I would like to express my sincere gratitude to the members of the jury.

I gratefully acknowledge the generous scholarship support of Fundação para a Ciência e Tecnologia (Portugal).

Thank you Prof. Pedro Amores da Silva for being the co-director at the begging of this $\mathrm{PhD}$, for helping me with the first contacts at University of Montréal and for your trust and support.

Furthermore, I would like to thank all the students of the laboratory of Professor Cartilier, in particular to Fabien Brouillet for all the well appreciated support, and to Salma Abdelaziz for helping me with the sampling in the early-morning hours, allowing me to avoid spending 36 hours or more with no sleep, but also to Shadi Moghadam and Hong Wei Wang for their kind patience to answer my questions.

I thank the co-authors of the papers produced during this $\mathrm{PhD}$ for their collaboration, in particular to Soula Kyriacos who was responsible for the clinical tests.

I would also like to thank all the members of the other laboratories of the faculty, in particular to Prof. Hildgen and to Jean Michel Rabanel for the endless help in the purchase of materials. 
I thank the administrative and support staff of the Faculty, especially Mrs. Andrée Mathieu, Lucie Racine and Josée Desrochers for facilitating my work both administratively and technically, and always with a smile.

Thank you so much to all my great friends in Montreal for making all these years so special and fun and for putting up with my bad temper.

Also, I would like to thank Jeanne Leblond Chain and all the students and members of the Group Meeting Axe Formulation for proofreading my papers and for their helpful suggestions.

Last but not least, I wish to express my deepest gratitude to my parents for their endless love and support. 


\section{Chapter 1. Introduction}

\subsection{Pharmaceutical dosage forms intended to oral drug administration}

\subsubsection{General principles}

The general objective of the administration of a pharmaceutical dosage form is to deliver an adequate dose of drug to a pharmacological target, so that a predictable therapeutic response to the drug (or drugs) included in the dosage form, which can be to prevent, cure or control a determined diagnosed pathological state, can be achieved. There are numerous dosage forms into which a drug can be incorporated in order to achieve a suitable and effective treatment of a disease.

The first requirement to take into consideration when formulating a new oral dosage form is a good knowledge of the pathway and the vast anatomical and physiological dissimilarities that the dosage form and the drug it contains will encounter during their passage throughout the gastrointestinal tract and until the drug reaches its target, so that an adequate pharmacokinetic and pharmacodynamic profile is attained. Moreover, the physicochemical and biological properties of the drug, the design of the dosage form, the drug-release mechanism and the particular disease factors are other factors that can influence the bioavailability of a drug and interact with one another. Dosage forms are also subject to intraand inter-patient variability. Therefore, the delivery of an adequate dose of drug to a pharmacological target remains a major challenge in the development of new pharmaceutical dosage forms.

Dosage forms are constituted of two main components: pharmaceutical drugs (or active ingredients) and excipients (also called auxiliary substances or additives). A dosage form can comprise one or several drugs (Le Hir, Chaumeil et al. 2009). A pharmaceutical drug is a substance intended to exert a pharmacological activity. In addition to being used in the treatment and prevention of a determined disease, a drug can also be used in the medical diagnosis of a disease. The excipients are the components in the dosage form, or used in its 
manufacturing process, which do not possess any pharmacological activity. Initially, the excipients were merely inactive substances used as vehicles or diluents for drugs and/or included in a pharmaceutical dosage form for varied and specialized pharmaceutical functions such as preserve, solubilise, suspend, modify dissolution, improve the compressibility, thicken, flavour and/or colour drug products (York 2002). Above all, they aim to improve the physicochemical properties of the drug in the dosage form, and ensure that the drug substance remains active and stable for a sufficiently long period of time. However, excipients have progressed and, although they are still necessary for their original functions, some are used nowadays in the control of drug action, contributing, as a result, to the improvement of drug efficacy in the human body.

Dosage forms can be designed for administration by diverse routes of administration to maximize therapeutic response. Depending on the route of administration and the type of formulation, different excipients may be used. The numerous excipients used in the formulation of dosage forms can also be explained by the large variety of physicochemical characteristics of drugs (Le Hir, Chaumeil et al. 2009).

The relationship between the drug, its dosage form and the route by which it is administered governs how much of the drug and how fast it enters the systemic circulation (Ashford 2002). Some routes, such as the parental routes (intravenous, intramuscular, intrathecal, subcutaneous...), imply the irruption of physiological barriers, and others such as the oral, the topical and the transmucosal (ocular, nasal, buccal, pulmonary, rectal, vaginal...) routes, among many others, do not imply the irruption of any physiological barriers.

The present thesis is focused on the development and evaluation of a novel excipient for oral sustained-release (SR) dosage forms. Besides the oral route, the intravenous route was also used in a clinical study using human volunteers. Therefore, only these two routes, and in particular the oral, will be described.

If a drug is given intravenously it is administered directly into the bloodstream, and thus the entire amount of drug administered reaches the systemic circulation and is available for distribution into other body tissues and the site(s) of action of the drug. On the other hand, if a drug is administered by a route other than the intravenous, there is no guarantee that the entire dose will reach the systemic circulation, because all routes of administration where a systemic action is required, apart from the intravenous, involve the absorption of the drug into 
the blood from the route of administration (Ashford 2002). Nonetheless, the intravenous route has its disadvantages, for instance, patients are not typically able to self-administer, it passes most of the natural defences of the body, and it can carry fatal air boluses.

Among the routes of administration that require an absorption step, the oral route is by far the most popular for administration of drugs intended for systemic action because it presents certain advantages (Le Hir, Chaumeil et al. 2009), which will be described in the next section.

\subsubsection{Oral drug administration}

Compared with other routes of administration, the oral route is the simplest, the most natural and convenient for the patient (non-invasive), and usually the safest and least expensive means of drug administration (Kokate, Marasanapalle et al. 2005; Le Hir, Chaumeil et al. 2009). The oral route also allows the administration of high doses of drug with only a single administration (Aiache, Aiache et al. 2001). It is estimated that currently about $90 \%$ of pharmaceutical dosage forms are administered orally (Kokate, Marasanapalle et al. 2005), being about $80 \%$ of the pharmaceutical forms administered by the oral route solid dosage forms (tablets, capsules...) (Jivraj, Martini et al. 2000). Although oral dosage forms are essentially intended for absorption into the bloodstream through the various epithelia and mucosa of the gastrointestinal tract, a few are destined to produce a local effect in the tract, such as a temporarily increase of the $\mathrm{pH}$ of the stomach.

The pharmacokinetic properties of the oral route of administration are the result of the great variability of environmental conditions and obstacles that the drug will encounter along the gastrointestinal tract, during its passage through the gastrointestinal membrane into the bloodstream as well as during its passage through the liver. In fact, the relatively slow onset of action after administration, the possibilities of reduced and irregular absorption and the biodegradation of certain drugs by the enzymes and secretions of the gastrointestinal tract constitute some of the disadvantages of this route of administration (York 2002). Other disadvantages are a potential altered bioavailability caused by drug and food interactions, the modification of the contents and microflora of the gastrointestinal tract by unabsorbed drug 
and the occurrence of eventual nausea or gastrointestinal tract discomfort due to drugs that cause local irritation (Shargel, Wu-Pong et al. 2004).

The progression of a drug in the body is a dynamic process that leads to the therapeutic effect of the same. After oral ingestion, the dosage form moves along the oesophagus towards the stomach. Then, it remains in the stomach until it can pass the pylorus and be transferred to the small intestine, where most of drugs are absorbed. The distinct phenomena that take place in the gastrointestinal tract after drug administration and may influence the bioavailability of drugs administered orally must be taken into consideration when designing a new oral dosage form. Indeed, in order to have the desired pharmacotherapeutic effect, it is necessary that the dosage form be able to deliver the drug to the section(s) of the gastrointestinal tract where it can be absorbed and assure that the drug is available in the body in a form that permits its absorption and passage into the systemic circulation. However, many factors can contribute to a decrease of the bioavailability of an orally administered drug. These factors include anything that adversely affects the release of the drug from the dosage form, its dissolution into the gastrointestinal tract fluids, its stability in solution in the same fluids, its passage through and stability in the gastrointestinal tract membrane, and its passage through the liver into the systemic circulation (Ashford 2002). After crossing the gastrointestinal tract membrane, drugs pass into the hepatic portal system and through the liver before they are transported via the bloodstream to its target site. Consequently, the amount of an oral dose of drug that reaches the systemic circulation can also be decreased by first-pass or presystemic metabolism by the gastrointestinal tract membrane and/or the liver. The factors contributing to a decrease of the amount of an orally administered drug that reaches the systemic circulation will be detailed in a following section.

The different stages between drug administration and the attainment of the therapeutic effect are generally described as a series of three phases: the biopharmaceutical phase, the pharmacokinetic phase, and the pharmacodynamic phase (Figure 1.1). 


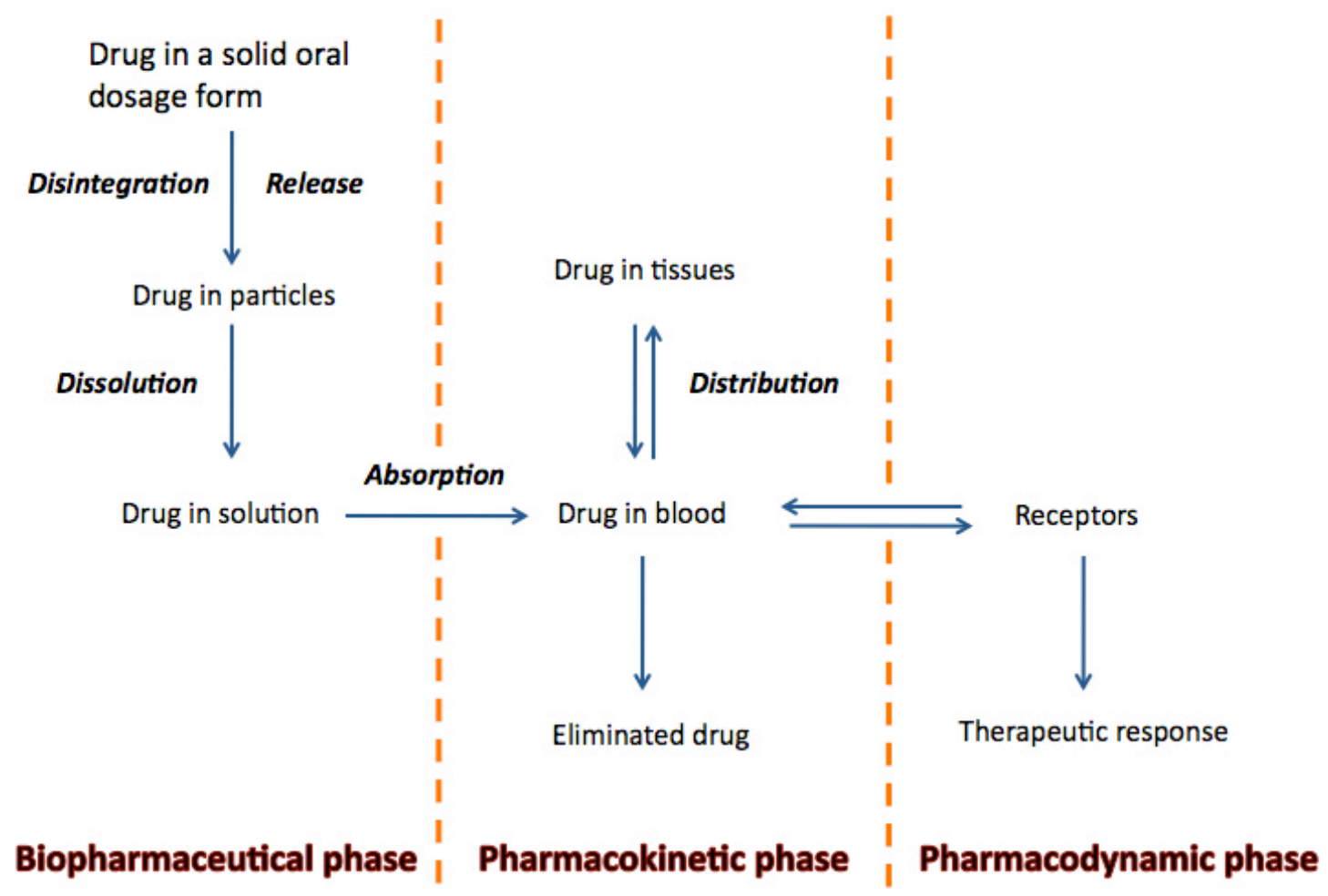

Figure 1.1 Representation of the three phases between drug administration and the attainment of the therapeutic effect: the biopharmaceutical phase, the pharmacokinetic phase, and the pharmacodynamic phase.

\subsubsection{Pharmaceutical oral dosage forms}

The dosage forms administered orally can be classified into two major categories (Aiache, Aiache et al. 2001):

1. Liquid forms, which include solutions, syrups, suspensions and emulsions.

2. Solid forms, which include tablets, powders, granules, pills, capsules (hard and soft gelatine capsules) and suppositories (suppositories may be also classified as semi-solid dosage forms).

The most popular oral dosage forms are tablets, capsules, suspensions, solutions and emulsions (York 2002). Although tablets and capsules are more widely used than liquid preparations for oral administration, the latter possess some advantages relatively to solid 
dosage forms. These advantages include, among others, the immediate availability for absorption, as the drug must be in solution to be absorbed, leading to a faster therapeutic effect, if required. Liquids are easier to swallow than solids and are, therefore, particularly suitable for paediatric and geriatric use. In addition, the administration of a solution of a drug is less likely to cause irritation and damage of the gastric mucosa by certain drugs because of the immediate dilution by the gastric contents (Billany 2002). However, these dosage forms also present some disadvantages, compared with solid dosage forms, which are related, mostly, to the storage, transport and stability of the ingredients in aqueous solution (both drugs and excipients), which are more susceptible to hydrolysis and to growth of microorganisms in solution, and to the administration of solutions, i.e., taste and lower accuracy of each dosage (Billany 2002).

Tablets are the most common solid dosage forms, and contain each a single dose of one or more drugs as well as one or more excipients. They are obtained by compression of uniform volumes of a mixture of powders held within a confined space. Depending on the type of tablet, they can be swallowed in one piece or split, chewed, dissolved or dispersed in water before administration, or retained in the mouth, where the drug is released (Alderborn 2002; Kottke and Rudnic 2002). Tablets present several advantages, including (Jivraj, Martini et al. 2000; Aiache, Aiache et al. 2001; Alderborn 2002; Ashford 2002):

1. It represents a convenient and safe way of drug administration, leading to an improvement of the patient compliance.

2. It is relatively easy to manufacture and transport solid oral dosage forms, as they do not need to be sterilized, are compact, and can be produced in large quantities by automated machines, allowing robust and quality-controlled production procedures at a relatively low price. Tablets can also be prepared in a versatile way with respect to their use and to the delivery of the drug.

3. Oral solid dosage forms, especially the ones in the dry and condensed form, are generally physically and chemically more stable, allowing a longer stability of the comprised ingredients, including drugs.

4. The preparation procedure enables accurate dosing of the drug per single dose and administration of larger amounts of drug in a restricted volume.

5. They are suitable for SR preparation. 
When a solid dosage form is administered orally, the first step to make the drug available is its release from the dosage form. This release can be immediate or modified. The drug-release mechanism states the dissolution of the drug and, consequently, has an effect on its absorption and pharmacokinetics.

\subsubsection{Drug-release from oral solid dosage forms: immediate versus modified drug-release}

The drug-release characteristics of a dosage form generally have a major effect on the therapeutic efficacy of the delivered drug. Based on their drug-release characteristics, tablets can be classified into two main types, immediate-release (IR) and modified-release (MR).

\subsubsection{Immediate drug-release dosage forms}

IR or 'conventional' tablets are designed to release the complete dose of drug contained within the dosage form, immediately following administration, i.e., the goal of this formulation is fast and complete drug-release in vivo, providing only a single and transient burst of drug. The released drug is assumed to be in a form that is therapeutically active and immediately available for absorption into the systemic circulation (Alderborn 2002; Collett and Moreton 2002). The fast release of drugs from IR tablets is achieved by fragmentation of the whole structure of the dosage form. When the tablet comes into contact with the fluids of the gastrointestinal tract, the liquid wets the solid and penetrates its pores. Consequently, the tablet disintegrates into granules or aggregates of primary particles, which subsequently deaggregate into their primarily drug particles. A deaggregation directly into fine particles will set up conditions for the fastest dissolution of the drug (Leblanc, Aiache et al. 1997; Alderborn 2002; Hoener and Benet 2002). However, drug dissolution can start before the complete fragmentation of the tablet. After the drug is in solution in the gastrointestinal tract, absorption may take place. The rate-limiting step in the dissolution process can be either the rate of disintegration of the dosage form and the size of the resulting aggregates or the deaggregation 
process (Hoener and Benet 2002). A disintegrant is usually included in the formulation of IR tablets to ensure that the tablet, when in contact with a liquid, breaks up into small fragments, promoting rapid drug dissolution. The two main mechanisms of action of disintegrants are (Alderborn 2002; Kottke and Rudnic 2002):

1. The promotion of liquid penetration into the pores of the tablet, resulting in the tablet breaking into fragments.

2. The induction of the rupture of the tablet by swelling of the disintegrating particles when in contact with liquids, by formation of gas or by enzymatic action. The smaller the primary particles obtained are, i.e., the larger the effective surface area, the faster will be the dissolution of the drug.

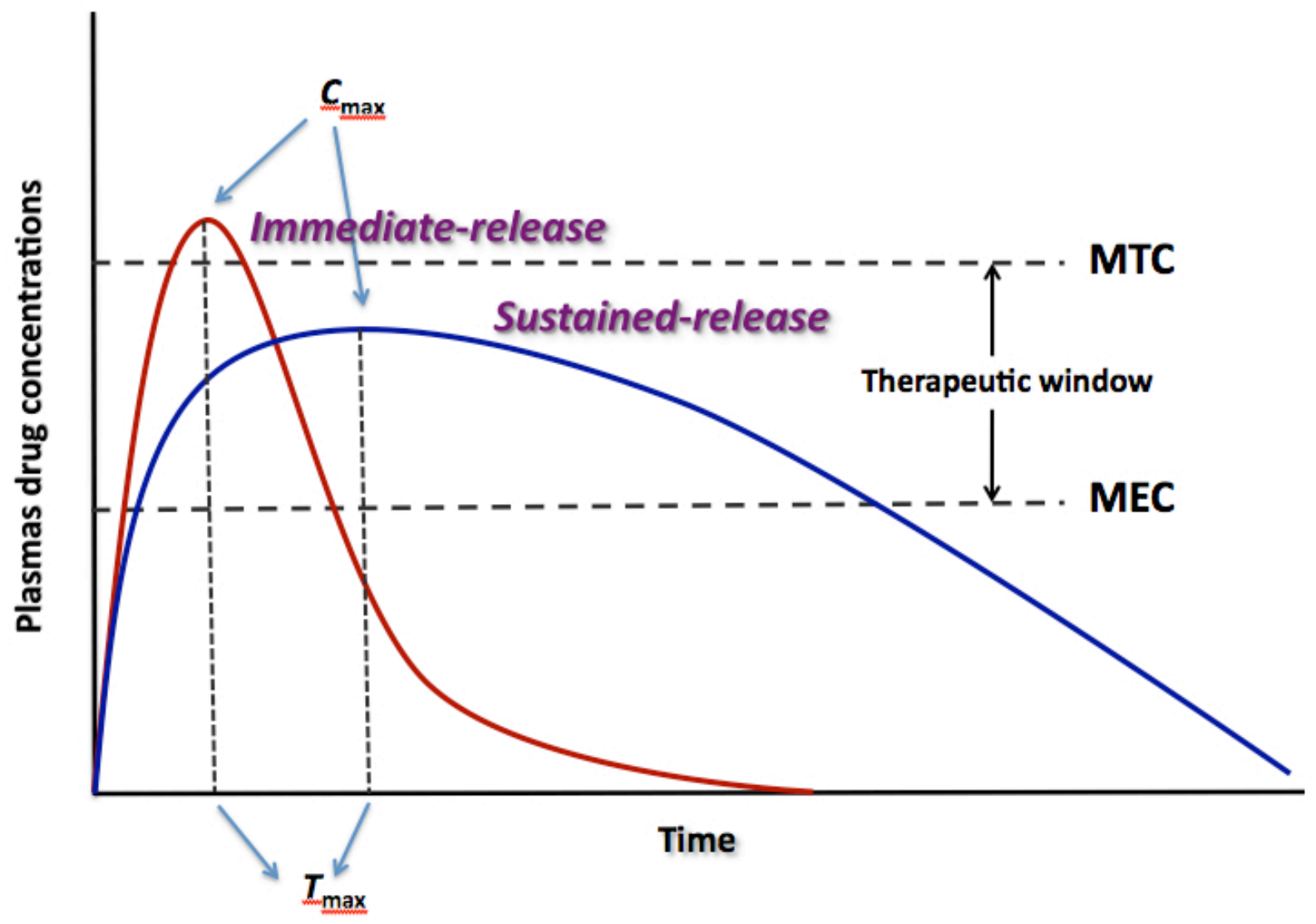

Figure 1.2 Difference between the plasma drug concentrations versus time profiles for immediate-release and sustained-release dosage forms. 
The pharmacokinetic behaviour of IR dosage forms consists generally of a fast increase of the plasma drug concentration until a maximum is attained (high maximum concentration, $C_{\max }$, and short time to reach the maximum concentration, $T_{\max }$ ) followed by the decaying of the plasma concentration corresponding to the elimination phase.

For most drugs there is an optimum plasma concentration range, usually known as therapeutic window or therapeutic range within which desired therapeutic effects are achieved. The therapeutic window lies between the MEC and the MTC (Figure 1.2), which represent the minimum effective (or therapeutic) concentration and minimum toxic concentration of drug, respectively. The minimum effective concentration is the minimum concentration of drug that has to be achieved in the plasma for the desired therapeutic or pharmacological effect to be elicited. The minimum toxic concentration is the concentration of drug in the plasma above which side effects or toxic effects occur.

For many diseases, the ideal dosage regimen is that by which an optimum therapeutic concentration of drug in the blood plasma, and hence at the site(s) of action of the drug, is attained immediately and then maintained as stable as possible within the therapeutic window for the desired duration of the treatment. Provided dose size and frequency of administration are correct, plasma concentrations of a certain drug lying within its therapeutic window can be achieved promptly and maintained by means of multiple administrations of IR oral dosage forms (Collett and Moreton 2002). Nonetheless, this dosage regimen has a few limitations. When IR dosage forms are administered, the plasma concentration-time profile of a drug fluctuates over successive dosing intervals, even when the 'steady-state' condition (when the overall rate of drug supply equals the overall rate of drug elimination from the body) is achieved. As long as the amount of drug is above the minimum effective concentration, a pharmacological response is observed. However, it is not possible to maintain a stable drug concentration within the therapeutic window for the duration of treatment. In addition, these fluctuations can lead to a patient being undermedicated or overmedicated for periods of time. The first situation happens if, following the oral administration of equal doses of a drug, the interval between each administered dose is longer than the time required for complete elimination of the previous dose. In this case, the plasma concentration-time profile corresponds to a series of isolated single-dose profiles and the plasma concentration only lies within the therapeutic window of the drug for a relatively short period following the 
administration of each dose. On the other hand, if the time interval between successive equal doses is less than that required for complete elimination of the previous absorbed dose, overmedication leading to eventual toxicity may occur due to accumulation of the drug in the body and plasma following successively administered doses (Proudfoot 2002). These limitations are even more significant for drugs with short biological half-lives, because of their more frequent dosing constraint, which makes it more difficult to keep plasma concentrations within the therapeutic window, especially when the therapeutic window is very narrow. An example of such a drug is theophylline, which has a narrow therapeutic window and a relatively short half-life in children (Kadlec, Ha et al. 1978). Furthermore, poor patient compliance is more likely to happen in the case of regimens requiring frequent administration, and is often an important reason for therapeutic inefficiency or failure ( $\mathrm{Li}$, Robinson et al. 1987; Collett and Moreton 2002).

\subsubsection{Modified drug-release dosage forms}

The limitations and requirements regarding the administration of IR dosage forms led pharmaceutical scientists to put a vast amount of work into designing MR dosage forms, which are intended to provide the drug at more precise temporal and spatial placement within the body. The United States Pharmacopeia definition of MR system is: "the one for which drug-release characteristics of time course and/or location are chosen to accomplish therapeutic or convenience objectives not offered by conventional dosage forms..."

Some authors have suggested two main distinct classifications within the MR dosage forms: sustained-release (referred herein as SR) dosage forms and controlled-released (referred herein as CR) dosage forms. According to this terminology, SR denotes any dosage form designed to maintain therapeutic plasma or tissue concentrations of the drug for an extended period of time by providing drug at a decreased release rate and, thus, slowing down the absorption rate. The $C_{\max }$ attained after a single administration of a SR dosage form will be usually lower than that attained after the administration of a single IR dosage form containing the same amount of drug, and the $T_{\max }$ will be longer compared to the IR dosage form (Figure 1.2). $\mathrm{CR}$, on the other hand, indicates that the delivery system is able to provide some actual 
therapeutic control, whether this be of a temporal nature, spatial nature, or both; that is, it attempts to control drug concentrations in the target tissue or cells. These definitions suggest that SR systems, which only prolong therapeutic blood or tissue concentrations of the drug for an extended period of time, cannot be considered as CR systems ( $\mathrm{Li}$, Robinson et al. 1987; Jantzen and Robinson 2002). However, there has been considerable confusion regarding the terminology of these dosage forms (Li, Robinson et al. 1987; Buri and Doelker 1997; Jantzen and Robinson 2002).

Thus, CR may be considered to include a broad number of objectives such as, among others, 1) to increase drug solubility and dissolution rates in order to improve absorption and, as a result, bioavailability; 2) to prolong retention in the stomach in order to control not only the rate of release but also the site of release; 3) to protect drugs and bioactive agents, such as peptides, proteins and enzymes, from degradation within the gastrointestinal tract; 4) to protect the stomach from the irritating effects of some drug by means of, for example, enteric coatings; and 5) to deliver drugs to their absorption window or to specific targets within the gastrointestinal tract, often the colon (site-specific) (Li, Robinson et al. 1987; Ranade 1991).

The main goals of oral SR products, on the other hand, can be described as follows ( $\mathrm{Li}$, Robinson et al. 1987; Ranade 1991; Gupta and Robinson 1992; Collett and Moreton 2002; Das and Das 2003; Rajabi-Siahboomi 2003; Kumar, Bhowmik et al. 2010):

1. To attain a therapeutic concentration of drug in the plasma (within the therapeutic window of the drug) quickly, which provides a fast onset of the desired therapeutic response in the patient, and, then, to maintain the concentrations within the therapeutic window, with a maximum reduction of fluctuations, for a longer period of time after the administration of a single dose. The decreased fluctuations of plasma concentrations result in more uniform and prolonged clinical effects with lower incidence and/or severity of systemic and local side-effects, better treatment of diseases where symptoms exacerbate if the plasma concentration of drug drops below the minimum effective concentration, and better overnight maintenance of the therapeutic action.

2. To reduce dosage frequency, which generally improves patient comfort and compliance and may reduce the total amount of drug administered over the duration of treatment.

3. To possibly hinder the drug degradation rate by delaying disintegration or dissolution of the dosage form. 
However, some potential limitations have been described for oral SR dosage forms, specifically (Li, Robinson et al. 1987; Ranade 1991; Gupta and Robinson 1992; Collett and Moreton 2002; Das and Das 2003; Kumar, Bhowmik et al. 2010):

1. The time to attain therapeutic blood concentrations is longer when the dosage form is not designed to provide a first loading dose.

2. There may be possible increased variation in bioavailability after oral administration.

3. Unpredictable and poor in vitro-in vivo correlation (IVIVC) are often observed with such formulations, especially when the drug-release rate is very low.

4. Enhanced first-pass effect may take place for certain drugs.

5. There are constraints on the types of drugs that are suitable candidates for incorporation into oral SR formulations. These restrictions will be discussed in a following section.

6. These dosage forms usually contain a higher total amount of drug than the single dose administered in a conventional dosage form. Therefore, there is a significant risk of overdose if the form happens to be defective or misused, which may cause a "dose dumping", i.e., a release of a potentially toxic dose of drug as a bolus, resulting in undesired high plasma drug concentrations and potential toxicity.

7. Variable physiological factors, such as gastrointestinal $\mathrm{pH}$, enzyme activities, gastric and intestinal transit rates, food and severity of disease, which often influence drug bioavailability from conventional oral dosage forms, may also interfere with the precision of control of release and absorption of drugs from oral SR dosage forms. The achievement and maintenance of prolonged drug action depends on such control.

8. SR products, which tend to remain intact, may become lodged at some site along the gastrointestinal tract. If this occurs, slow release of the drug may produce a high-localized concentration that causes local irritation to the gastrointestinal mucosa.

9. There is reduced potential for dosage change or withdrawal in the event of toxicity, allergy, or poisoning.

10. There is limited dosage flexibility.

11. The gastrointestinal transit time of the SR dosage form along the gastrointestinal tract represents a physiological constraint to these pharmaceutical products because it limits the residence time at the absorption site and, consequently, the maximum period for which a therapeutic response can be maintained following administration of a single dose. 
12. SR dosage forms usually imply superior production costs than conventional dosage forms, mostly associated with the more complex production methodology used. However, the total cost of the treatment with SR products may be comparable to or even lower than the one with IR products. With a decrease in side effects, the overall expense in disease management may be reduced. Besides, therapies with SR products require less 'unit doses'.

13. Variable bioavailability is often observed. However, improved bioavailability has been described (Raber, Schulz et al. 1999; Rajabi-Siahboomi 2003).

For the purposes of this thesis, SR and CR will be considered as separate delivery processes as suggested.

A zero-order release from the dosage form, i.e., drug-release rate that is independent of the amount of drug remaining in the dosage form at any given time (constant release rate), has often been considered as ideal for SR forms. However, in practice, it is difficult to obtain a zero-order release. Thus, these dosage forms usually try to mimic zero-order release by providing drug at a slow first-order rate (i.e. concentration-dependent) (Jantzen and Robinson 2002).

The maintenance of constant plasma drug concentrations is also hindered by the presence of all the variable physiological conditions associated with the gastrointestinal tract. Consequently, even if a zero-order release from the dosage form is achieved, the drug absorption kinetics does not necessarily follow the same order of the release from the dosage form. Besides, to maintain a constant drug concentration in the plasma and the target tissue, the release rate from the dosage form and, thus, the absorption rate should be equal to the rate of elimination from the body ( $\mathrm{Li}$, Robinson et al. 1987). The rate of drug elimination from a tissue or from the body is, however, considered to be a first-order process (both metabolism and renal excretion) as well as the absorption and distribution of drug (Ranade 1991; Shargel, Wu-Pong et al. 2004; Shargel, Wu-Pong et al. 2004). Furthermore, the elimination kinetics of a given drug is also subject to inter and intra-patient variability depending on such factors as genetic differences, age differences and differences in the severity of disease, which further complicates the achievement of a constant drug concentration. 
SR dosage forms are often designed so as to release a first amount of drug (loading dose) into the gastrointestinal fluids immediately following administration of the dosage form, in order to attain the desired therapeutic response promptly. This first dose is followed by a slow release of the remaining dose of drug (the main one), thereby resulting in therapeutic drug concentrations that, although not remaining constant, decline at such a slow first-order rate that they remain within the therapeutic window for an adequately extended period of time (Li, Robinson et al. 1987; Ranade 1991; Collett and Moreton 2002).

For IR dosage forms, the rate-limiting step in drug bioavailability is usually the absorption of drug across the gastrointestinal wall, which has been described as a first-order kinetic process ( $\mathrm{Li}$, Robinson et al. 1987). When designing a SR or CR product, one aims for release of drug from the dosage form as the rate-limiting step instead. Thus, drug availability is controlled by the kinetics of drug-release from the dosage form rather than absorption. As a result, the associated rate constant(s) for drug-release are smaller than the absorption rate constant (Li, Robinson et al. 1987).

For the successful formulation of a SR dosage form it is necessary to take account of the physiology of the gastrointestinal tract, the physicochemical and the biological properties of the drug, the design of the dosage form, the mechanisms of drug-release (from the macroscopic effects of size, shape and structure to chemistry and molecular interactions), and the particular disease factors. All of these can influence or interact with each other (Collett and Moreton 2002). For example, the interaction between the physicochemical properties of a drug and the characteristics of the dosage form in which it is delivered determines the temporal release pattern that is observed ( $\mathrm{Li}$, Robinson et al. 1987). In addition, the rational design of SR dosage forms, where biological, physicochemical and physicomechanical considerations have been taken into account during formulation has decreased the risk of dose dumping in vivo.

The efficacy of SR compared to conventional formulations has been demonstrated for tramadol, the second model drug used in this study (Grond and Sablotzki 2004; Keating 2006). 


\subsection{Solid dosage forms for oral sustained drug-release}

\subsubsection{General principles}

The two most common formulation strategies employed to provide sustained drugrelease are to (1) embed the drug in an insoluble or swellable matrix with release-retardant materials or (2) enclose the drug or the dosage form in a SR coating.

The majority of SR systems rely on diffusion, dissolution (bioerosion), or a combination of both mechanisms, to generate slow release of drug to the biological environment (diffusion and/or erosion systems) (Hui, Robinson et al. 1987; Ranade 1991). Both formulation strategies described above provide a diffusion and/or erosion barrier to drugrelease. However, other approaches, such as osmotic pressure (osmotically controlled systems) and, more rarely, ion exchange (ion exchange systems), have also been used (Doelker 1985; Hui, Robinson et al. 1987; Jantzen and Robinson 2002; Kumar, Bhowmik et al. 2010). Indeed, one of the classifications proposed for the SR systems is based on the mechanisms controlling drug-release (Doelker 1985; Ranade 1991). All these type of systems are made of polymers (Kumar, Bhowmik et al. 2010).

Furthermore, oral SR drug delivery systems can be classified into two major groups: single-unit or monolithic dosage forms (e.g., large tablets or capsules) and multiple-unit dosage forms (e.g., pellets) (Bodmeier 1997).

\subsubsection{Multiple-unit dosage forms for sustained drug-release}

Multiple-unit dosage forms are those in which a single dose of a drug is administered subdivided into many small dosage units. For oral administration, sufficient units to comprise a single therapeutic dose are usually given enclosed in a hard gelatine capsule, a disintegrating tablet or in a sachet. These dosage forms are generally used as delayed-release or SR systems, though they may provide IR products, (for example, for reduced localized gastric irritation) (Melia, Washington et al. 1994). 
Multiple-unit dosage forms include preparation technologies such as drug-loaded pellets, often as coated spheroids, granules, mini-tablets and drug crystals (Rajabi-Siahboomi 2003). The two strategies used to provide SR mentioned above, i.e. insoluble or swellable matrices and SR coatings, have been combined within a single type of multiple-unit dosage forms (Melia, Washington et al. 1994; Collett and Moreton 2002).

These dosage forms offer some advantages in sustained drug-release. For example, multiple-unit dosage forms are considered to be less susceptible to the effects of food than the monolithic dosage forms (Rajabi-Siahboomi 2003). They can lead to decreased variability produced by food intake in the bioavailability of certain drugs due to more reproducible gastric emptying patterns when compared to single SR units above a certain size. The small size of each unit allows the passage through the pylorus independently of the motility cycles in the stomach, contrarily to the large monolithic forms, which are often retained, leading to variability in the gastric emptying patterns that are displayed both in fasted and fed subjects (Melia, Washington et al. 1994). Another relevant advantage of multiple-unit dosage forms is associated with a lower risk of toxicity caused by overdose. When a single monolithic dosage form happens to be defective or misused and, consequently, disintegrates within the gastrointestinal, it can potentially lead to dose dumping. Failure of a multiple-unit dosage form is unlikely to cause disruption of all the particulates, and thus the dosage released will be minimal. Multiple-unit dosage forms also reduce the likelihood of localized mucosal damage to the gastrointestinal tract, as the probability of many multiple-units becoming entrapped within, for example, a single mucosal fold in the stomach or intestine, is small.

However, these dosage forms also present disadvantages, which are mainly related to their manufacturing process. First of all, if the technology chosen is new to a pharmaceutical company, a high investment and significant time is generally required in their development and introduction. Unforeseen difficulties may occur in stability testing and quality control of the new product as a result of lack of a knowledge support and experience in the new technology. In addition, filling of the multiple-unit spheroids into capsules can be a problem owing to build-up of static charge. But, even if the technology and knowhow is well established, production costs and time for manufacture are often higher for multiple-unit dosage forms than for tablets. Indeed, monolithic tablets are associated to greatly efficient manufacturing technologies and much lower costs (Collett and Moreton 2002). 


\subsubsection{Monolithic dosage forms for sustained drug-release}

Oral single-unit or monolithic dosage forms include tablets of different types as, for example, coated tablets and matrix tablets, and some capsules. To facilitate their description, solid monolithic dosage forms for SR can be divided into three main groups: reservoir or membrane-controlled systems, matrix systems and osmotic pump systems (Collett and Moreton 2002). All these systems allow water penetration and, when they come into contact with a dissolution medium, drug diffusion will occur from a region of high concentration to a region of low concentration, i.e., down its concentration gradient. Initially, the interior of the system usually has lower water content than the surrounding medium and the concentration difference will be the driving force for drug diffusion out of any of these systems (Collett and Moreton 2002).

Among the many monolithic dosage forms that can be used for sustained drug-release, matrix tablets are of major interest in the pharmaceutical industry because of their highly efficient manufacturing technology.

\subsubsection{Reservoir systems}

Reservoir delivery systems, also called membrane-controlled systems, are constituted by a core of drug, the reservoir, surrounded by a polymeric membrane, usually water-insoluble (Figure 1.3). This membrane, through which the drug must diffuse, is the rate-controlling element of the system. The nature of the chosen polymer that forms the membrane determines the diffusion of drug and, hence, the rate of drug-release from the system (Collett and Moreton 2002; Jantzen and Robinson 2002). When a dissolution medium diffuses into the reservoir system, the membrane hydrates and becomes permeable. After hydration of the membrane, dissolution of drug at the interface core-membrane initiates drug diffusion and release. The drug diffuses then through the membrane down its concentration gradient, and is subsequently released at the interface membrane-external medium (Heller 1987). Unlike hydrophilic matrix systems, which will be discussed subsequently in this section, the membrane polymer does not usually swell and erode on hydration (Collett and Moreton 2002). However, SR systems with 
erodible rate-controlling membranes have also been described (Heller 1987). An initial loading dose can be achieved by formulating the system with a determined fraction of the dose in the membrane (Collett and Moreton 2002).

\section{Reservoir system}

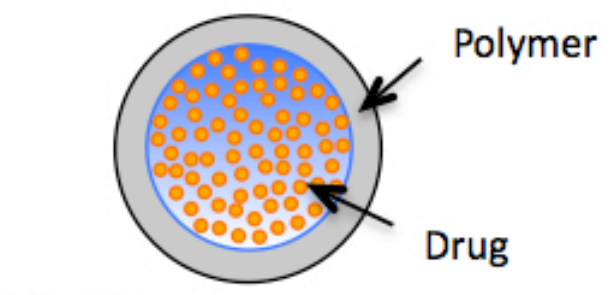

Time 0

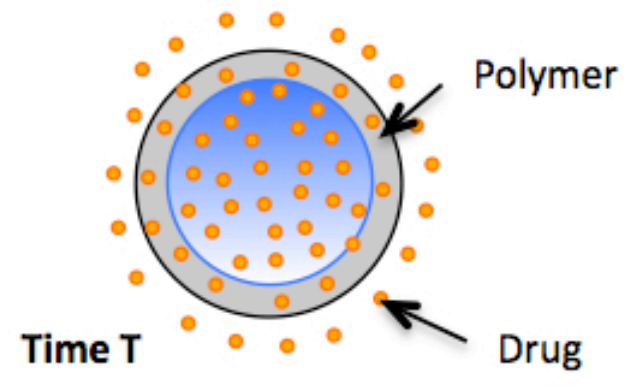

\section{Inert matrix}

Drug dispersed in polymer

Time 0

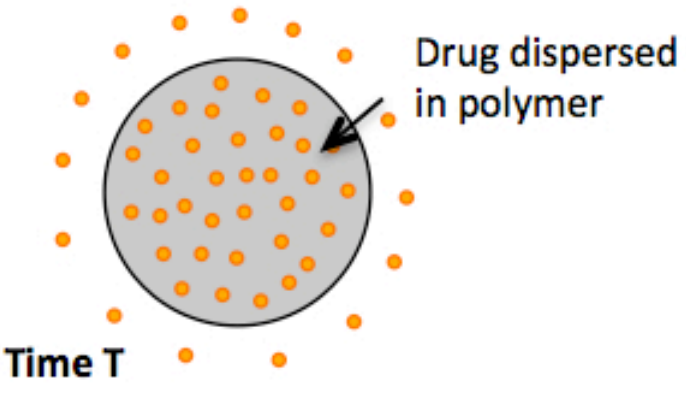

Figure 1.3 Diffusion of drug across the polymeric membrane of a reservoir system and across an inert matrix system.

The drug-release rate at time $t$ from reservoir systems with a non-erodible membrane, $d M_{t} / d t$, can be described by the following equation (Jantzen and Robinson 2002):

$$
\frac{d M_{t}}{d t}=\frac{A \cdot D \cdot K \cdot \Delta C}{d}
$$


where $M_{t}$ is the mass of drug released after time $t, A$ the surface area of the device (effective area of diffusion), $D$ the diffusion coefficient of the drug in the membrane in units of area/time, $K$ is the partition coefficient (the ratio of drug concentration in the membrane to that in the bathing medium at equilibrium), $\Delta C$ is the concentration difference across the membrane, and $d$ the thickness of the diffusion layer (the diffusional path length).

In theory, these systems should offer the possibility of zero-order delivery. However, zero-order release rate can be feasible only if all the variables on the right side of the equation remain constant. Consequently, these systems often fail to release drug at a constant rate, since it is particularly difficult to maintain all these parameters constant. However, it is possible to modulate the kinetics of release from these systems by changing one or more of these parameters, in particular, by changing the characteristics of the polymeric membrane, like its nature and/or its thickness. These modifications allow, ideally, the adjustment of the kinetics of release in order to improve the therapeutic efficacy of the drug (Jantzen and Robinson 2002).

Reservoir systems have some disadvantages inherent to their own structure. For example, unless the polymer used is soluble, the system must somehow be removed from the body after the drug has been released. Another critical aspect to consider is the fact that, usually, the reservoir contains a large amount of drug. Therefore, any error in production or any accidental damage to the dosage form, like a membrane rupture, which would directly expose the reservoir core, could expose the patient to a potentially toxic dose of drug. This issue becomes especially important when designing reservoir systems for drugs with narrow therapeutic ranges or high toxicity (Jantzen and Robinson 2002).

Although, for convenience of description the reservoir systems were included as part of the monolithic dosage forms for SR, some multiple-unit reservoir systems have been developed where the coated drug reservoir is, in this case, a multiparticulate pellet instead of a tablet (Collett and Moreton 2002). 


\subsubsection{Matrix systems}

A matrix system consists of drug dispersed or dissolved homogenously throughout a polymer matrix. This matrix is resistant to its immediate disintegration and controls the release of drug. Therefore, whereas in the case of a reservoir system the polymeric membrane that controls the release of drug is only at the surface of the system, in a matrix system the ratecontrolling polymer is throughout the whole system. In both cases, it is the hydration of the polymer and the consequent dissolution of the drug that allow the drug to diffuse out of the system (Collett and Moreton 2002).

Compared with the reservoir systems, the matrix systems present several advantages, which are the reason why they are the most employed systems in the control of drug-release. Some of the main advantages are the following (Jantzen and Robinson 2002):

1. Since the total drug constituent is dispersed in a continuous and unique matrix, the risk of dose dumping is less likely to occur and, hence, these systems offer a higher safety level.

2. Matrix systems are much easier and cheaper to produce, usually by direct compression.

Like the reservoir systems, unless the polymer used is soluble, the remaining matrix 'ghost' must be removed from the body.

The classification of the different types of matrix systems (Figure 1.4) usually takes into account several criteria, such as the structure of the matrix, the release kinetics, the mechanisms controlling drug-release, and the chemical nature and properties of the materials forming the matrix (Buri 1987). Drug diffusion, erosion and/or swelling of matrix are the main mechanisms by which this type of systems can control the release of drug. The predominance of one mechanism over the other will depend on the properties of the polymer used to produce the system. 

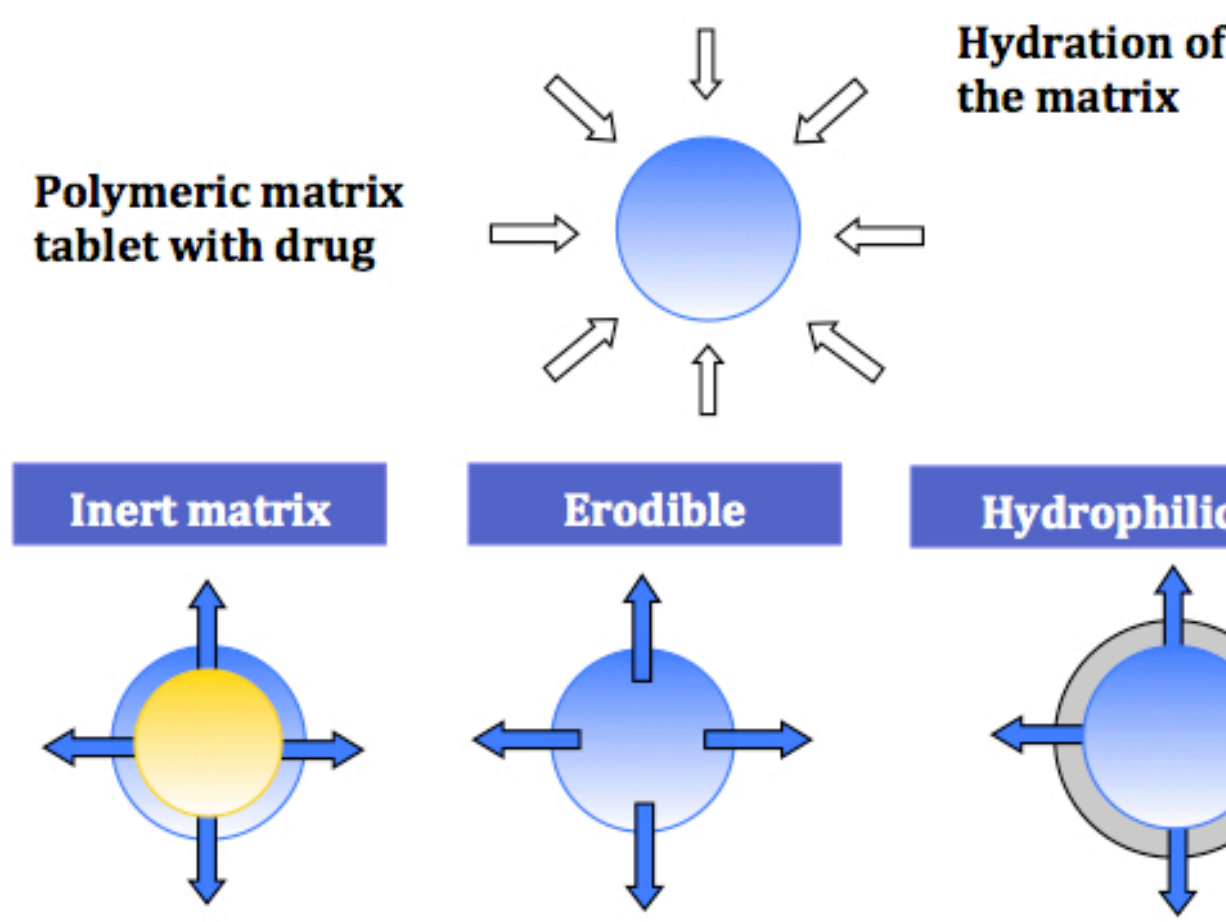

\section{Hydrophilic matrix}

\section{Drug release}
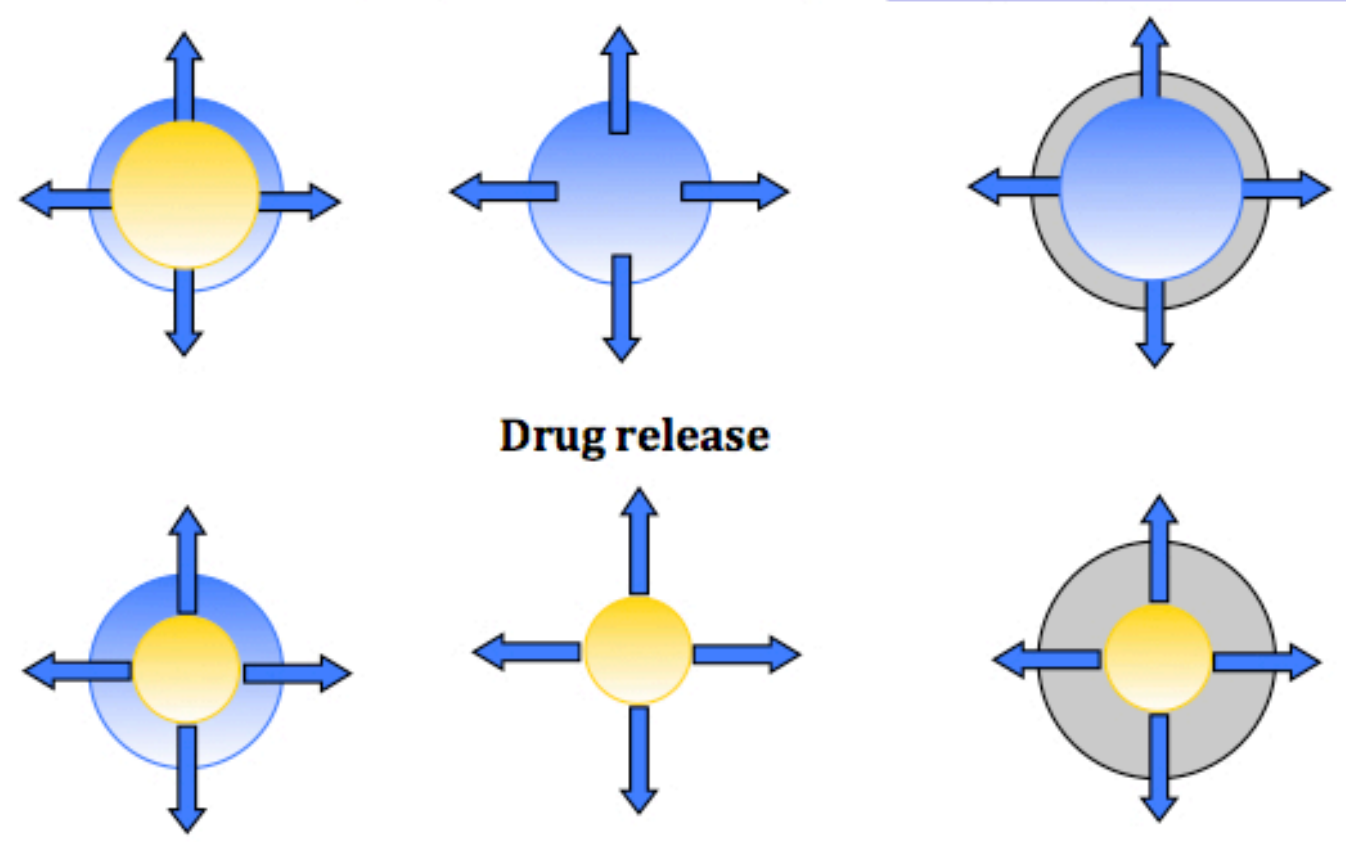

Figure 1.4 Drug-release in function of the type of matrix system. The blue represents the initial size of the matrix with drug; the yellow represents the region of the polymeric matrix with the remaining drug; the gray represents the swollen matrix; and the arrows represent the diffusing drug.

\section{Erodible polymer matrices}

A method used to decrease the release rate of a drug with high water-solubility and, therefore, fast dissolution rate is to incorporating it into a matrix tablet whose main excipient 
will erode slowly when in contact with the fluids of the gastrointestinal tract. In this case, the rate of drug diffusion in the polymer is slow compared to the polymer dissolution (Katzhendler, Hoffman et al. 1997) and, therefore, dissolution of the polymer is the ratelimiting step. In actual fact, therapeutic systems are never dependent on dissolution only or diffusion only. However, in practice, SR systems have been classified as either dissolutioncontrolled or diffusion-controlled when the predominant release mechanism overshadows other mechanisms (Jantzen and Robinson 2002).

In SR matrix tablets employing dissolution as the rate-limiting step, drug-release is controlled by the gradual dissolution (bioerosion) of the polymeric matrix containing the drug or by hydrolysis or enzymatic reaction of a bond that attaches the drug directly to the polymer (Ranade 1991; Jantzen and Robinson 2002). Moreover, two different modes of erosion have been described: surface (heterogeneous) and bulk (homogeneous) erosion. In bulk-eroding systems, erosion occurs homogeneously throughout the bulk of the system. In surface-eroding systems, on the other hand, erosion is confined to the outer surface of the system (Heller, Baker et al. 1978; Heller 1980). The rate of drug-release from a surface-eroding system is determined by the relative contribution of the drug diffusion and the erosion of the matrix (Katzhendler, Hoffman et al. 1997).

The mathematical description of the release characteristics from erodible systems is complex. One of the difficulties relates to the fact that erodible systems combine dissolution of both the polymeric matrix and the drug, and diffusion of drug out of the system after dissolution. As the polymer dissolves, the diffusional path length for the drug may change. Zero-order release can occur only if surface erosion occurs and surface area does not change with time (Jantzen and Robinson 2002). In addition, in the case of a drug compressed with a slowly dissolving polymer into a matrix tablet, the rate of drug delivery is controlled by the rate of penetration of the dissolution medium into the matrix, which can be, in turn, controlled by the porosity of the tablet matrix, the eventual presence of hydrophobic additives, the wettability of the tablet and the particle surface (Heller 1987). Nevertheless, a model was developed to describe drug-release from matrices of various geometries showing only surface erosion and for which the erosion process is the rate-limiting step (Katzhendler, Hoffman et al. 1997): 


$$
\frac{M_{t}}{M_{\infty}}=1-\left(1-\frac{k_{0} \cdot t}{C_{0} \cdot a_{0}}\right)^{n}
$$

Equation 2

where $M_{t}$ is the amount of drug released from the system at time $t, M_{\infty}$ is the total amount of drug released when the system is exhausted, and $k_{0}$ is the erosion rate constant. $C_{0}$ is the uniform initial concentration of drug in the matrix, and $a_{0}$ is the initial radius for a sphere or cylinder or the half-thickness for a slab (geometry of a tablet). The exponent $n$ depends on the geometry of the system with $n=1$ for a slab, $n=2$ for a cylinder, and $n=3$ for a sphere.

A major advantage of these systems compared with non-erodible matrices is that, as the matrix erodes entirely, the problem related to the removal of the dosage form from the body does not apply in this case. However, a particular attention must be given to the degradation products, as it is essential that they are not toxic, carcinogenic nor teratogenic.

\section{Inert matrix systems}

Inert matrix systems, also called insoluble matrices, are constituted of drug particles dissolved or dispersed in a matrix constituted mainly of a polymer insoluble in the gastrointestinal fluids (Figure 1.3). When an aqueous medium penetrates the insoluble matrix, its structure remains unaffected. The release takes place by simple diffusion of dissolved drug from the system along the network of fluid-filled pores, cracks and intergranular spaces of the system (Higuchi 1963; Collett and Moreton 2002).

The release rate of a drug from an inert matrix will be mainly dependent on the structure of the matrix, in particular, its total porosity and its tortuosity, i.e., its pore structure, but also on the properties of the drug such as its solubility.

The amount of drug released from an inert system with connecting capillaries can be determined by the following equation proposed by Huguchi (Higuchi 1963): 


$$
M=\left[\frac{D \varepsilon}{\tau}\left(2 \cdot A-\varepsilon \cdot C_{s}\right) \cdot C_{S} \cdot t\right]^{1 / 2} \quad \text { Equation } 3
$$

where $M$ is the amount of drug released at time $t$ per unit exposed area, $D$ is the coefficient of diffusion of the drug in the permeating fluid, $\tau$ is the tortuosity factor of the capillary system, $A$ is the total amount of drug present in the matrix per unit volume, $C_{S}$ is the solubility of the drug in the permeating fluid, and $\varepsilon$ is the porosity of the matrix.

If the release is ruled only by pure diffusion, the quantity of drug released from matrix dosage forms is usually directly proportional (linear correlation) to the square root of time of exposure to the dissolution medium (Collett and Moreton 2002), as given by the equation:

$$
M_{t}=k \cdot t^{1 / 2}
$$

Equation 4

where $M_{t}$ is the amount of drug released at time $t$, and $k$ is the kinetics constant.

A zero-order release is not possible with this type of matrices, as the rate of drugrelease decreases with time of exposure to the dissolution medium. Initially, the diffusion pathway that the drug molecules at the surface of the matrix have to traverse is very short. As the dissolution process progresses towards the interior of the matrix, the depletion area increases and, as a result, the diffusion pathway increases in length. In addition, the area of drug exposed to dissolution medium decreases.

Most of the factors that affect the release of drug from an inert matrix are, somehow, related to the capacity of the dissolution medium to penetrate the matrix. The main factors are the following (Buri and Doelker 1997; Sajeev and Saha 2001; Collett and Moreton 2002):

1. The compression force $(\mathrm{CF})$ - an increase of the $\mathrm{CF}$ is normally correlated with a decrease of the porosity of the matrix. A more rigid and less porous matrix will, generally, release drug more slowly than a more porous matrix.

2. The particle size of the insoluble matrix components - smaller particles usually lead to a less porous and more tortuous matrix and, thus, a decreased release rate. 
3. The concentration of the inert matrix components - when the concentration is high, the total porosity (addition of the initial porosity to the porosity created by the dissolution of the drug) decreases, which results in a decrease of the release rate. The initial porosity is an important factor that can have an influence in the penetration of the dissolution medium and, consequently, in the dissolution of the drug in the matrix.

4. Drug loading - an increase in drug loading tends to augment the release rate, but the relationship between the two is not clearly defined (Collett and Moreton 2002), although release rate is, normally, related to drug solubility.

5. The affinity of the inert support for the dissolution medium - the more wettable the inert polymer used is, the higher will be the penetration rate of the medium and the higher will be the release rate.

6. The nature of the other excipients in the formulation - usually, water-soluble excipients will enhance the release rate as a result of an increase in the wettability of the matrix and in its porosity after the dissolution of the excipient. Insoluble substances, on the other hand, will tend to decrease the penetration of the dissolution medium and, thus, the release rate (Collett and Moreton 2002).

This type of system is not suitable for the release of drugs that are insoluble or that have a low aqueous solubility, given that this a totally diffusional system and that the use of this type of drugs will result in a weak driving force of diffusion generated by a too low concentration of dissolved drug. If the quantity of water that can penetrate the matrix is not enough to solubilise the entire amount of drug, the total dose will not be available ( $\mathrm{Li}$, Robinson et al. 1987; Collett and Moreton 2002).

\section{Lipophilic matrix systems}

Like the inert matrices, the lipophilic matrix systems contain drug particles dispersed in an insoluble matrix, with drug becoming available as an aqueous medium penetrates the matrix and dissolves the particles. These systems are compact wax matrices prepared from blends of powdered components using standard direct compression, roller compaction or hot- 
melt granulation. After entering the matrix, the medium dissolves a channelling agent, which was added to the formulation. The dissolved channelling agent leaches out of the system, leading to the formation of a porous matrix of tortuous capillaries. The drug dissolves in the aqueous medium and diffuses out of the matrix through the water-filled capillaries. The hydrophobic matrix remains intact during drug-release.

The main advantage of this type of system is related to their easy manufacturing with no need of a lubricant as the waxes act themselves as a liquid-film lubricant during compression. Nevertheless, they offer a rather coarse control of rate and extent of drug-release (Collett and Moreton 2002).

\section{Hydrophilic matrix systems}

In recent years the value of hydrophilic polymer-based matrix tablets as vehicles for SR delivery has been increasingly demonstrated with the publication of numerous patents and research papers and their utilization in new products in the pharmaceutical market. The widespread and successful use of such polymeric systems can be attributed to the fact that they are simple to formulate, relatively inexpensive, easy to produce and generally regarded as safe, they have shown favourable in vivo performance with a more precise control of drug delivery in the fluids of the gastrointestinal tract, resulting in reproducible profiles and better IVIVC, they are versatile in controlling the release of drugs with a wide range of physicochemical properties, and they have superior patient acceptability (Collett and Moreton 2002; Durig and Fassihi 2002).

The hydrophilic matrix systems are monolithic systems prepared by compression of a powdered mixture of a hydrophilic polymer (swelling agent), one or several drugs, and other excipients, if necessary. Those matrices can be also denominated swellable matrix systems.

As opposed to the inert matrices, the structure of a hydrophilic matrix responds to the presence of water or biological fluids, which change the polymer structure with the time of exposure to the aqueous medium, enabling the drug to be released from a dosage form.

Since the dosage forms developed in the work of this dissertation are hydrophilic matrix systems, these will be discussed in more detail in a following section. 


\subsubsection{Osmotic pumps}

Although osmotic pump systems are described in the present work as a distinct type from reservoir systems, osmotic pumps are, in a sense, another form of reservoir delivery systems. The essential difference between the two types of systems is that in the case of osmotic pumps only one diffusion process is required, which is the passage of aqueous medium into the core through the membrane, whereas in the classic reservoir system two processes are involved: the diffusion of aqueous medium into the system and the diffusion of drug out of the system. Therefore, in the case of osmotic pumps the diffusing species is only water.

The elementary osmotic pump (Figure 1.5) consists of a tablet containing a solid core of drug and an agent that is soluble enough to provide the high osmotic pressure difference, such as an electrolyte, surrounded by a semipermeable membrane. The membrane is permeable to water but not to drug, and has single or multiple holes drilled through it to allow drug-release. When this device is exposed to water or any body fluid, water will flow into the core owing to the osmotic pressure difference, and will dissolve it. As the core dissolves, a hydrostatic pressure builds up and pumps drug solution (or suspension) out of the system through the hole (Collett and Moreton 2002; Jantzen and Robinson 2002).

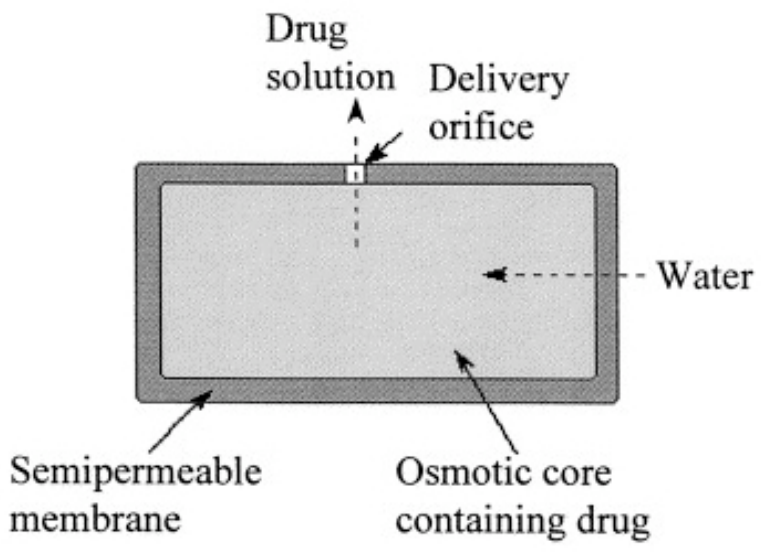

Figure 1.5 Elementary osmotic pump with a solid osmotic core, a semipermeable membrane and, in this example, a single hole drilled through the membrane. Figure reproduced from (Simó, Cifuentes et al. 2003) with permission of Elsevier Ltd. 
Other types of osmotic pumps have been described as, for instance, a system consisting of a drug layer containing the drug and excipients and a push layer containing osmotically active components (Figure 1.6). This osmotically active bilayer core is enclosed in a semipermeable tablet shell membrane that is permeable to water but not the drug or osmotic components. As water is absorbed from the gastrointestinal tract at a rate determined by membrane properties and the osmolality of the core constituents, the push layer expands and presses on the drug layer, slowly releasing drug solution/suspension through a laser-drilled orifice in the tablet shell (Palangio, Northfelt et al. 2002).

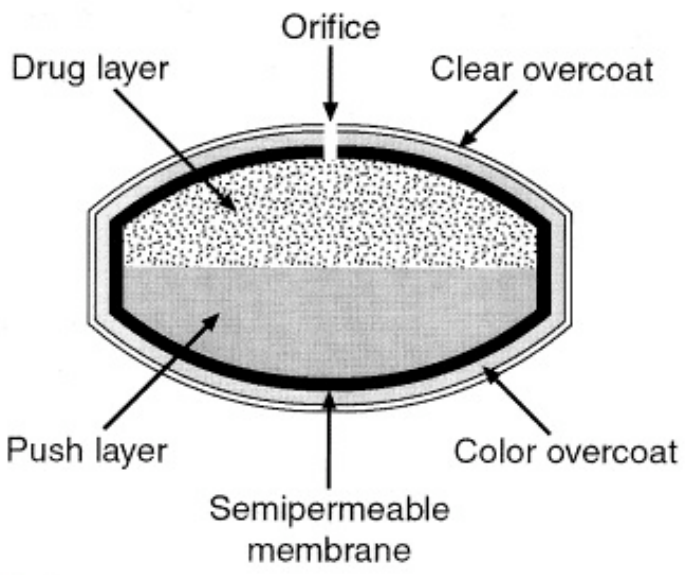

Figure 1.6 Osmotic pump consisting of an osmotically active bilayer core enclosed in a semipermeable tablet shell membrane, which is permeable to water, but not the drug or osmotic components, and has a laser-drilled orifice on the drug-layer side of the tablet. The bilayer core is comprised of a drug layer and a push layer. Figure reproduced from (Palangio, Northfelt et al. 2002) with permission of Elsevier Ltd.

Another important factor governing the drug-release rate from osmotic systems, besides the rate at which water is able to pass in through the membrane, is how quickly the drug solution/suspension can pass through the hole. Therefore, and the size of the hole(s) is critical (Collett and Moreton 2002). 
The release of drug from these systems is in theory not dependent on the drug (Bayne, Place et al. 1982 ) and, therefore, they are suitable for a wide range of drugs, which are normally difficult to deliver by the solution-diffusion mechanism, such as poorly watersoluble drugs. Since the osmotic gradient remains constant, osmotic pumps can give a theoretically zero-order release profile after an initial lag time, which then gradually falls to zero (Collett and Moreton 2002; Palangio, Northfelt et al. 2002). With systems such as the osmotic pump, dissolution rate may be rendered independent of gastrointestinal variables, such as pH and viscosity (Gupta and Robinson 1992). However, these systems can lead to dose dumping if there are any defects in the coating membrane. Besides, they are relatively expensive and require rigorous quality control (Collett and Moreton 2002).

\subsubsection{Ion exchange systems}

Ion exchange systems consist of resins composed of water-insoluble cross-linked polymers. These polymers contain salt-forming functional groups in repeating positions on the polymer chain. The drug is bound to the resin and released by exchanging with the ions in the gastrointestinal tract, when these are in contact with the ion-exchange groups. The rate of drug diffusing out of the resin is controlled by the area of diffusion, diffusional path length, and the rigidity of the resin, which is a function of the amount of cross-linking agent used to prepare the resin. These systems offer protection of the drug to the enzymes in the gastrointestinal tract by temporarily altering the substrate. However, the release of drug is proportional to the concentration of ions present in the area where the drug is ought to be released, which is affect by variability in diet, water intake, and intestinal content (Jantzen and Robinson 2002). 


\subsubsection{The special case of hydrophilic matrix systems}

Hydrophilic matrix systems are monolithic dosage forms prepared by compression and constituted by a homogenous dispersion of drug molecules within a frame in which one or several of the excipients incorporated are a hydrophilic polymer. Some examples of these polymers will be given in section 1.2.5.1. These systems are currently the preferred strategy for producing sustained release solid oral dosage forms (Melia 1991; Mikac, Kristl et al. 2011).

The most important factors to be taken into account when developing a formulation based on hydrophilic matrices are the percentage, solubility and drug particle size, the type of polymer, the percentage incorporated, its degree of viscosity and the polymer particle size, the drug/polymer ratio and the amount of water entering the matrix. The CF used to produce the matrices can be important because it determines matrix porosity. Other factors have shown to be involved in the release of drugs, such as the dimensions of the matrix and, in the case of mixtures of polymers, their percentages (Maderuelo, Zarzuelo et al. 2011).

\subsubsection{Drug-release mechanisms from hydrophilic matrix systems}

The most important aspect of the release mechanisms from matrix systems constituted by hydrophilic polymers is the formation of a gel layer around the matrix, owing to a change of state of the polymer in response to water penetration (Figure 1.7). When a matrix containing a swellable glassy polymer comes into contact with an aqueous medium, such as the gastrointestinal fluids, there is an abrupt change of state, which corresponds to a transition from the glassy, a rigid and compact state, to the rubbery state, an expanded and flexible state. The individual polymer chains absorb water and the molecules of water insert themselves between the polymeric chains. The presence of water reduces the glass transition temperature $\mathrm{T}_{\mathrm{g}}$ of the polymer (which is controlled by the concentration and the type of swelling agent, and depends on the temperature and the thermodynamic interactions of the polymer-water system) to the system temperature T (Colombo, Bettini et al. 2000; Mikac, Kristl et al. 2011). This process induces a reduction of the polymer-polymer intermolecular attraction forces and, 
consequently, an increase in the distance between the chains and in their mobility, i.e., a polymer-chain relaxation. The higher chain mobility favours the transport of dissolved drug and permits chain reorganization in the form a three-dimensional gel. Therefore, as a result of the glassy-rubbery polymer transition, an outer gel layer forms on the matrix surface and the matrix increases in volume because of swelling. The gel layer acts as a barrier against the fast release of drug while controlling, at the same time, the penetration of aqueous medium and the diffusion of drug.

The formation and the characteristics of the gel layer are crucial parameters regarding the control of drug-release from gel-forming matrices. If the polymer gels slowly, the dissolution medium can penetrate deep and quick into the glassy matrix and disintegrate the matrix before gel layer formation occurs. Therefore, it is essential that the polymer used in the manufacture of these matrices is able to form the gel layer quickly and that the 'protective' layer maintains its stability during the release process (Colombo, Bettini et al. 2000).

Initially, when the aqueous medium penetrates into the matrix through its pores, the drug present on the surface of the matrix is released quickly while the polymeric chains rearrange to form the viscous gel layer surrounding the dry core of the tablet. Here, the polymer chains are strongly entangled and the gel layer is highly resistant. The penetration of water continues then towards the centre of the matrix, but it is now slowed down by the progressively forming gel barrier. The formation of this gel layer also leads to a progressive reduction of the release rate of dissolved drug due to an increase of the diffusion path length (Chien 1982). To certain polymers, as the gel layer becomes progressively hydrated, the quantity of absorbed water can be such that the interactions water-polymer can become superior to the interactions polymer-polymer. When sufficient water has accumulated, the chains disentangle and the polymer dissolves (Lee and Peppas 1987; Narasimhan and Peppas 1997). This causes the appearance of progressive erosion on the surface of the matrix. 
Solid matrix tablet containing the drug

\section{In an aqueous medium}
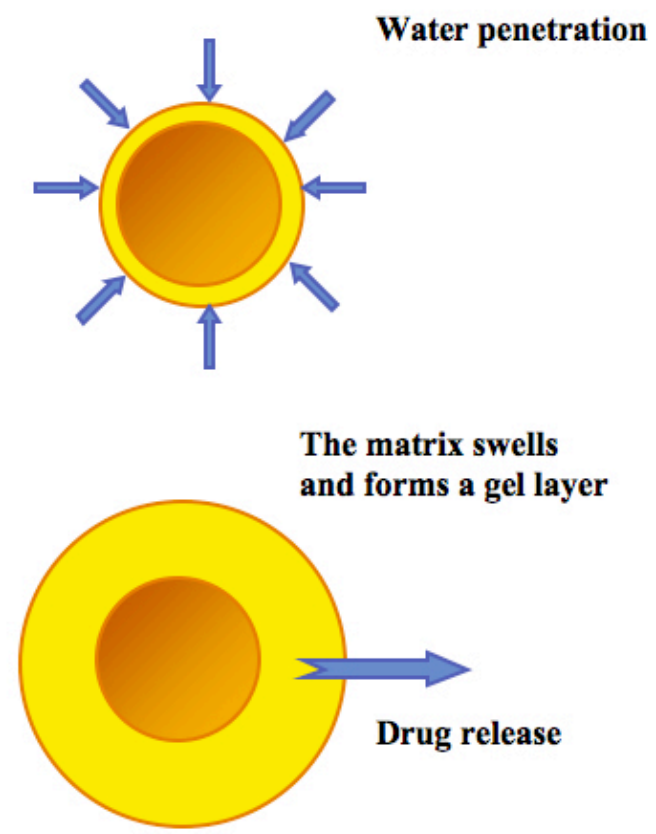

Figure 1.7 Schematic representation of drug-release from hydrophilic polymer matrix tablets.

The mechanical behaviour of the swelling process, which is a result of the relaxation of polymeric chains, is characterized by the presence of different fronts, where 'front' indicates the position in the matrix where the physical conditions change abruptly (Figure 1.8) (Colombo, Bettini et al. 1995). 


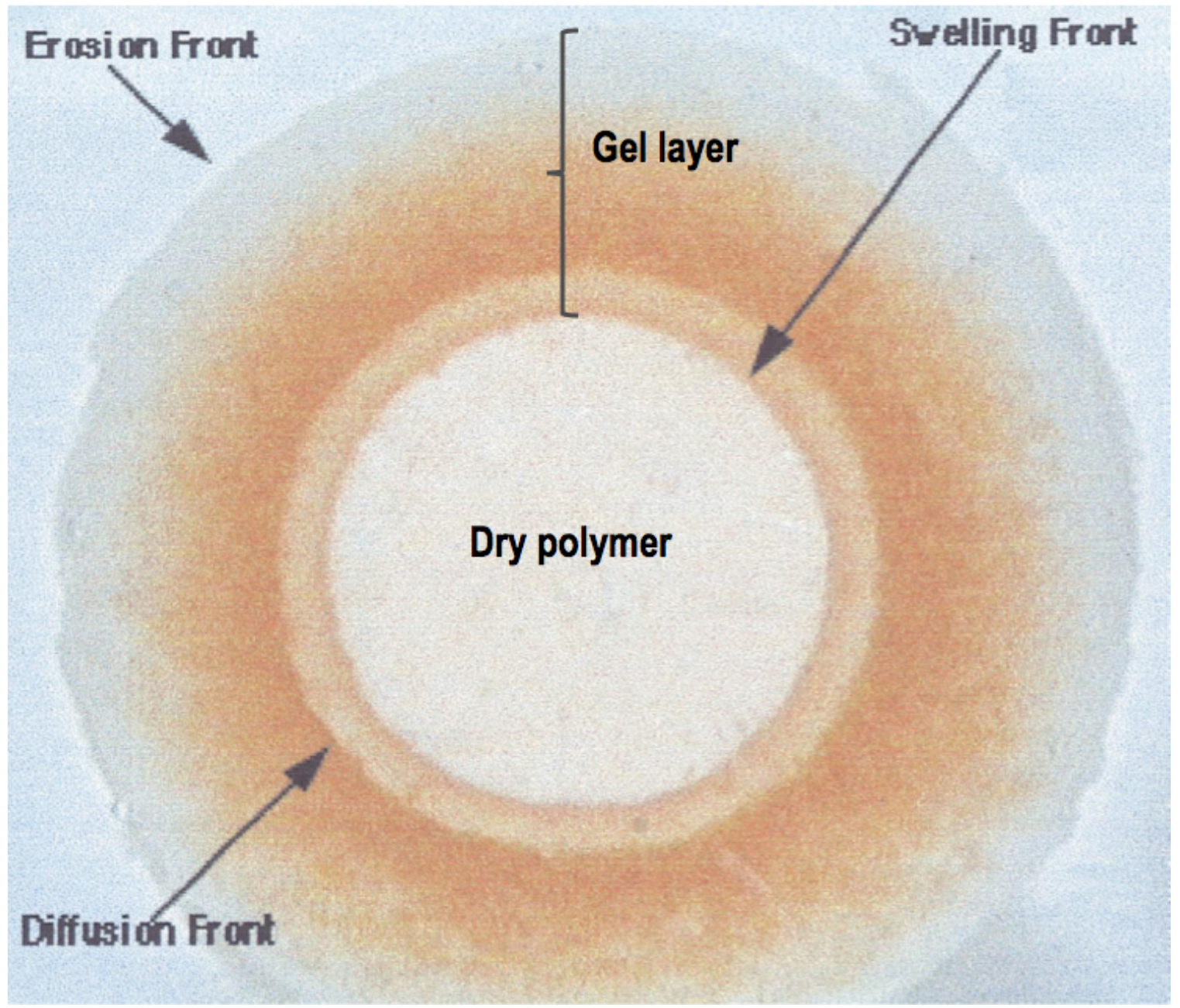

Figure 1.8 Fronts in the swelling process (swelling, diffusion and erosion) of hydrophilic matrices during drug-release. The behaviour of the gel layer was studied using a colorimetric technique. HPMC matrices containing soluble and coloured buflomedil pyridoxal phosphate were prepared by wet granulation. Swelling studies were performed by clamping each matrix between two transparent Plexiglas ${ }^{\circledR}$ disks and the assembled device was introduced into the vessel of a USP 23 apparatus 2 containing distilled water $\left(37^{\circ} \mathrm{C}, 200 \mathrm{rpm}\right)$. At fixed times during swelling, the device was taken out of the vessel and pictures of the disc matrix base were video-recorded. The front distance was then measured in pixels and these were converted into length units. Figure adapted from (Colombo, Bettini et al. 1999) with permission of Elsevier Ltd. 
From the periphery to the centre of the matrix, the first observed front is the 'erosion front', which corresponds to the boundary between the surface of the matrix and the dissolution medium. It is in this region of the matrix, where the maximum separation and solubilisation of chains occur, that the drug is released. A second front, the 'diffusion front' has been identified (Lee and Kim 1991). This front constitutes an interface that separates undissolved from dissolved drug. During drug-release, the diffusion front position in the gel phase is dependent on drug solubility and loading and its movement related to drug dissolution rate in the gel (Colombo, Bettini et al. 1995). The diffusion front is located between the erosion front and the boundary where the glassy polymer transforms into the rubbery polymer, called the 'swelling front'. Some authors described a forth front, referred to as the 'penetration front', which separates the dry glassy polymer from the hydrated glassy polymer, that is, the position of the penetration of the medium into the dry glassy polymer (Brazel and Peppas 2000; Colombo, Santi et al. 2000; Ferrero, Bruneau et al. 2000). The positions of the different fronts within the gel are related to three important parameters of the swelling/dissolution process: the rate of water penetration, the rate of drug dissolution and the rate of erosion of the polymeric matrix (Colombo, Bettini et al. 1995).

Besides being exposed to continuous changes in its structure, the gel layer is also subject to changes in its thickness. The gel layer thickness as a function of time (Figure 1.9) is described as the distance between the relative position of the swelling and erosion moving fronts (Colombo, Bettini et al. 2000). In addition, the gel layer thickness dynamics exhibits, generally, three different phases during drug delivery. Initially, the thickness increases when solvent penetration is the fastest mechanism. Then, it remains constant when the water penetration and the disentanglement occur at a similar rate, leading to synchronization between the phenomena of swelling and erosion of the polymeric chains. Finally, the gel layer thickness decreases when the entire polymer has undergone the glassy-rubbery transition and the only process still active is the erosion (disintegration) of the polymer network (Lee and Peppas 1987). 


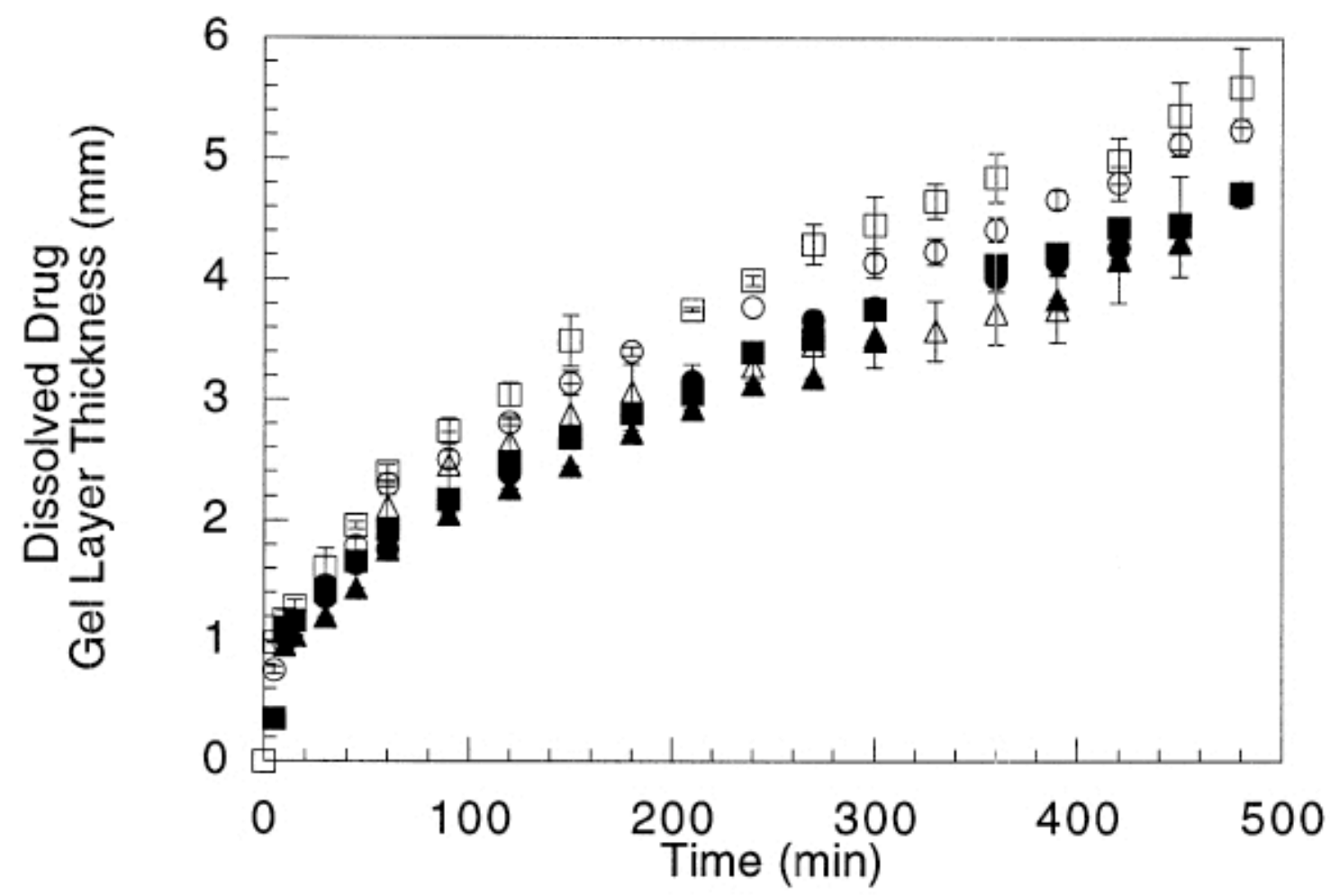

Figure 1.9 Gel layer thickness as a function of time for HPMC matrices containing different percentages of buflomedil pyridoxal phosphate, using the same conditions of Figure 1.8. Figure reproduced from (Colombo, Bettini et al. 1999) with permission of Elsevier Ltd.

Drug-release depends on the dynamics of gel layer thickness in the sense that an increase of thickness is inversely related to the release rate, owing to the increase of the diffusion path length.

A dissolved drug gel layer thickness, which corresponds to the distance between diffusion and erosion fronts, has also been described. It was shown that, though the kinetics of drug-release depends on the relative position of the three fronts, this section of the gel is the reference element for drug-release, instead of the whole gel layer thickness (Colombo, Santi et al. 2000). 
Several authors have proposed different analytical techniques, such as Calorimetry, Light Microscopy and Nuclear Magnetic Resonance (NMR), to characterize the matrix swelling process and the effects involved in drug-release (Gladden 1994; Gao, Skoug et al. 1996; Colombo, Bettini et al. 1999; Baille, Malveau et al. 2002; Richardson, Bowtell et al. 2005; Thérien-Aubin, Baille et al. 2005; Mikac, Kristl et al. 2011; Wang, Assaad et al. 2011).

In summary, the drug-release mechanisms and kinetics from hydrophilic matrices depend on phenomena that govern gel layer formation and dynamics, that is, solvent penetration into the matrix, polymer hydration and swelling (relaxation process), drug dissolution and diffusion through the gel layer, and matrix erosion. The control of drug-release is obtained by diffusion of molecules through the gel layer that can erode. The rate of drug diffusion depends on drug dissolution and matrix erosion, both affecting the drug concentration gradient in the gel layer (Colombo, Santi et al. 2000). A convective contribution to drug transport in the gel layer due to the relaxation of polymer chains was also described (Colombo, Bettini et al. 1999).

\subsubsection{Mathematical analysis of drug-release from hydrophilic matrix systems}

Even though the control of drug-release is usually mainly obtained by drug diffusion, the release kinetics do not necessarily follow the square root of the time as in the case of inert matrices, where the release is exclusively controlled by pure diffusion. A large spectrum of mathematical models describing drug-release from the hydrophilic matrix tablets has been developed. These models try to include many parameters that affect drug-release and kinetics, such as the thickness of the gel layer and the position of the different fronts (Colombo, Bettini et al. 1999; Kazarian and van der Weerd 2008), the presence of different excipients (Pillay and Fassihi 2000; Baumgartner, Pavli et al. 2008), the properties of the selected polymer and drug (Talukdar, Michoel et al. 1996; Saša, Odon et al. 2006; Ferrero, Massuelle et al. 2010), the polymer concentration across the gel, the mesh size of the polymeric network (Baumgartner, Kristl et al. 2002), and so on. However, in many cases, the use of a simple empirical or semiempirical model is sufficient (Siepmann and Peppas 2001). 
A semi-empirical equation, proposed by Peppas et al. (Peppas 1985) has been extensively used to describe drug-release from these systems:

$$
\frac{M_{t}}{M_{\infty}}=k \cdot t^{n}
$$

Equation 5

Where $M_{t}$ and $M_{\infty}$ are the absolute cumulative amount of drug released at time $t$ and infinite time, $M_{t} / M_{\infty}$ is the fraction of drug released, $k$ is a constant incorporating structural and geometric characteristics of the device (with units of $T^{n}$ ), $t$ is the release time, and $n$ is the release exponent, indicative of the mechanism of drug-release. The values of $n$ can vary between 0.43 and 1.00 , according to the geometry of the delivery system and the prevalence of diffusion (Fickian transport) or erosion (Case-II transport). Table 1.1 resumes the different $n$ values for three distinct geometric forms and the associated types of drug-release mechanism.

Table 1.1 Diffusional exponent for drug-release $(n)$ and associated drug-release mechanism from polymeric sustained delivery systems. Table reproduced from (Peppas and Sahlin 1989) with permission of Elsevier Ltd.

\begin{tabular}{|cccc|}
\hline \multicolumn{3}{|c|}{ Diffusional exponent $(n)$} & Drug-release mechanism \\
\hline Film & Cylinder & Sphere & \\
0.5 & 0.45 & 0.43 & Fickian diffusion \\
$0.5<\mathrm{n}<1.00$ & $0.45<\mathrm{n}<0.89$ & $0.43<\mathrm{n}<0.85$ & Anomalous transport \\
1.0 & 0.89 & 0.85 & Case-II transport \\
\hline
\end{tabular}

Fickian diffusional release corresponds to the molecular diffusion of drug through the hydrated outer gel layer due to a concentration gradient, which was described previously, and Case-II relaxational release is the drug transport mechanism associated with the relaxation/erosion of the polymer as a result of state-transition and swelling upon contact with water or biological fluids. The anomalous transport corresponds to the superposition of both phenomena, i.e., Fickian diffusion and Case-II transport. 
However, drug-release from swellable water-soluble polymer systems is typically described as an addition of these two simultaneously operating mechanisms. Therefore, a model that can identify the contributions of both phenomena to overall kinetics is preferable when analysing drug-release from hydrophilic/swellable matrices. Indeed, another empirical equation that considers both Fickian diffusional release and relaxation/erosion as additive was proposed (Peppas and Sahlin 1989):

$$
\frac{M_{t}}{M_{\infty}}=k_{1} \cdot t^{m}+k_{2} \cdot t^{2 m}
$$

Equation 6

where $k_{1}$ is the Fickian kinetic constant and $k_{2}$ is the relaxation/erosion kinetic constant (with units of $T^{n}$ ), and the first term of the right hand side represents the Fickian diffusional contribution (F), whereas the second term represents the Case-II relaxational contribution (R). The coefficient $m$ is the purely Fickian diffusion exponent for a system of any geometrical shape which exhibits sustained drug-release. Regardless of the geometry of the system used, the value of the exponent for Case-II transport mechanism is twice that of pure Fickian diffusional mechanism.

Both Eqns. 5 and 6 can be used to analyze the first $60 \%$ of a release curve, regardless of the geometric shape (Peppas 1985; Peppas and Sahlin 1989). 
The exponent $m$ can be determined by interpolation using a diagram also proposed by Peppas et al. (Peppas and Sahlin 1989), shown in Figure 1.10, which represents the values of $m$ in function of the Aspect Ratio, $2 a / l$ (where $2 a$ is the diameter and $l$ is the thickness of the tablet).

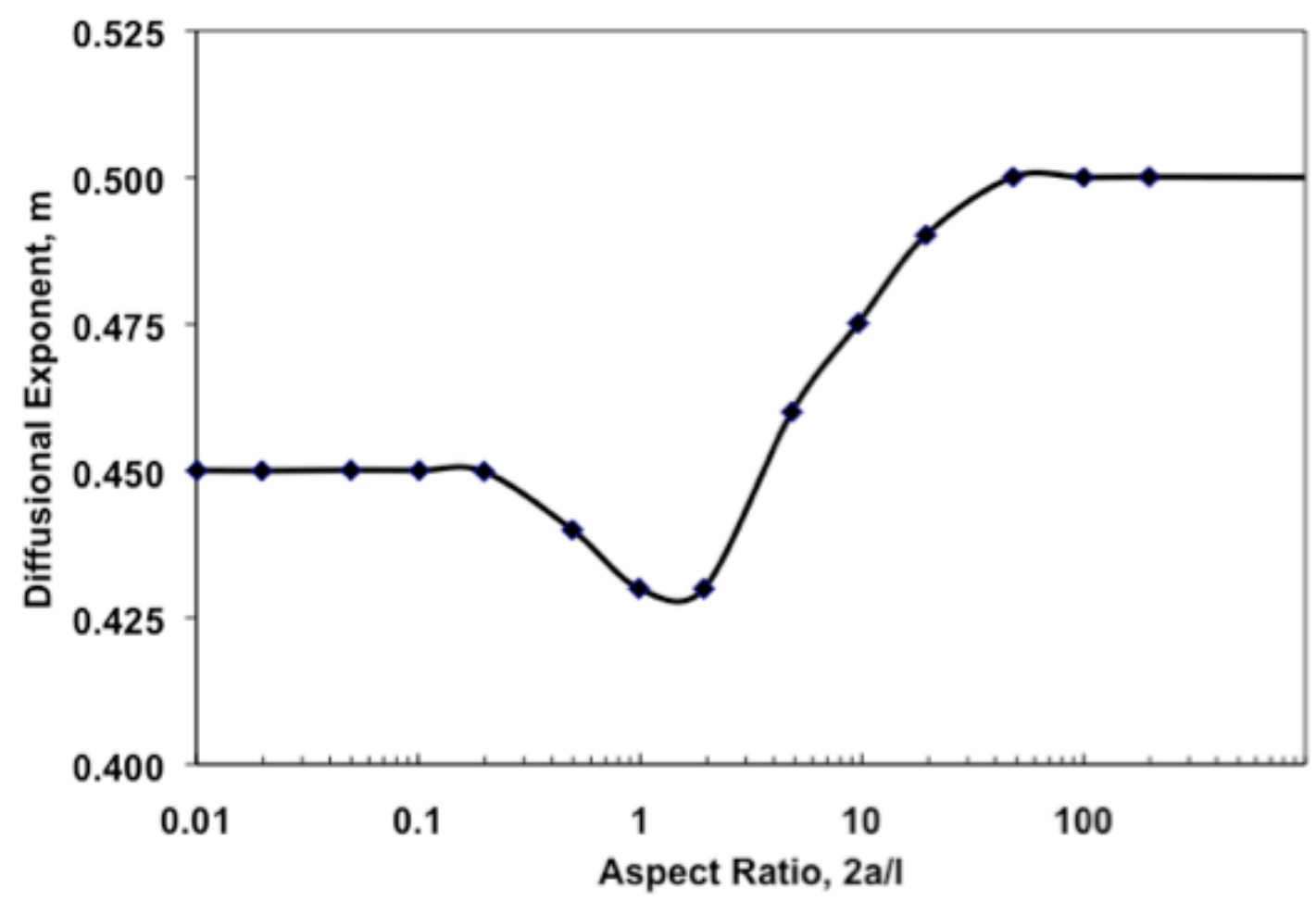

Figure 1.10 Variation of the Fickian diffusional exponent, $m$, with the aspect ratio, $2 a / l$, where $2 a$ is the diameter and $l$ is the thickness (height) of the device. Figure reproduced from (Peppas and Sahlin 1989) with permission of Elsevier Ltd.

Equation 6 can be written as (Peppas and Sahlin 1989):

$$
\frac{M_{t}}{M_{\infty}}=k_{1} \cdot t^{m}\left[1+\frac{k_{2}}{k_{1}} \cdot t^{m}\right]
$$

By comparison of Eqns. 6 and 7, it is concluded that $m=n$ when $\mathrm{F}$ is much superior to $\mathrm{R}$. The percentage of drug released due to the Fickian mechanism, F, is, in that case, calculated as: 


$$
F=\left[1+\frac{k_{2}}{k_{1}} \cdot t^{m}\right]^{-1}
$$

Equation 8

which leads to the ratio of the relaxational over Fickian contribution as follows:

$$
\frac{R}{F}=\frac{k_{2} \cdot t^{m}}{k_{1}}
$$

Equation 9

\subsubsection{Excipients used in hydrophilic matrix systems}

\subsubsection{Polymers for sustained drug-release systems}

The use of polymers for biomedical applications, especially in the pharmaceutical field, has been widespread for many years. These materials constitute the most important category of excipients employed in SR. They possess a large range of properties required for a precise control of drug-release and are easily adaptable to the usual manufacturing techniques of matrix tablets.

For a polymer to be effective in a pharmaceutical dosage form several of its properties must be taken into consideration. These properties include the solubility of the polymer in the biological fluids, the porosity, permeability, crystallinity and swellability of the polymer structure, its surface characteristics, its toxicity, and the diffusivity of drug molecules through the polymer material.

Among the polymers used in pharmaceutical applications, polysaccharides, like starch and cellulose, and their derivatives, are especially interesting for the production of hydrophilic matrices for oral SR dosage forms. Polysaccharides are abundant and inexpensive natural biomaterials, and are biocompatible and biodegradable. If the uses of these materials in the native state are varied, the possibility to produce derivatives has multiplied the possibilities. These modifications are made in order to obtain new properties or improve the initial properties of these polysaccharides. Unlike the expensive systems based on synthetic 
polymers, these semi-synthetic compounds have the advantage of being a base material easily obtainable. These advantages have made polysaccharides and their derivatives the materials of choice for the control of drug-release from matrix systems (Melia 1991).

Numerous polysaccharides have been studied in the development of hydrophilic matrix tablets. The studied polysaccharides include natural gums and materials, such as xanthan (Talukdar and Plaizier-Vercammen 1993; Talukdar, Michoel et al. 1996; Varshosaz, Tavakoli et al. 2006) guar (Khullar, Khar et al. 1998; Durig and Fassihi 2002; Varshosaz, Tavakoli et al. 2006) karaya, carrageenan (Raghavendra Rao, Sagar et al. 2009), dextran (Hennink, De Jong et al. 2004), sodium alginate (Giunchedi, Gavini et al. 2000 ), chitosan (Tu, Shen et al. 2011), and tamarind seeds (Sumathi and Ray 2002). However, cellulose and starch derivatives are the most commonly used compounds. Starch and its derivatives will be introduced in the next section, since the polymer used to produce the matrix tablets of the research of the present thesis was a modified starch.

The use of cellulose, an abundant naturally occurring biopolymer, and its derivatives as a binder or as agent for the MR of drugs has been well documented (Alderman 1984; Blaschek 1990; Doelker 1990; Doelker 1993; Kamel 2008). Typical modifications of cellulose are esterification and etherification of its hydroxyl groups. Most water-soluble and organic solvent-soluble cellulose derivatives are prepared by these substitution reactions, which can lead to radical changes in the original properties of cellulose (Kamel 2008). Some chemically modified cellulose derivatives, including water-soluble cellulose ether derivatives, specifically, sodium carboxymethyl cellulose (CMC), hydroxypropyl cellulose (HPC), hydroxyethyl cellulose (HEC) and hydroxypropyl methylcellulose (HPMC), have been used in solid SR formulations because of their swelling and gel forming capacity when in contact with an aqueous medium (Nakano, Ohmori et al. 1983; Rencher, Babu et al. 1995; Dabbagh, Ford et al. 1999). In fact, among a large number of natural and synthetic polymers that were tested as hydrophilic excipients for the preparation of SR matrix tablets, HPMC has been the most extensively studied. This significant use is due to the fast formation of a gel layer on the surface of HPMC matrices, which controls initial release, and to the high swellability and strong viscosity of the formed gel, which has a significant effect on the release kinetics of an incorporated drug. Besides, HPMC is compatible with numerous drugs. However, the gels formed show a continued expansion accompanied by erosion, which makes the transport 
mechanism relatively complex. Drug-release from HPMC matrices follows the mechanisms already described for hydrophilic matrices, i.e., drug diffusion through the swelling gel layer and release by diffusion out of the system, and matrix erosion of the swollen layer (Siepmann and Peppas 2001).

\subsubsection{Other excipients in the formulation of matrix tablets}

In the formulation of matrix tablets it is usually necessary to add excipients that improve the flow and compressibility properties of the powdered mixture formed by the drug and the polymer. The usual excipients used in solid dosage forms are listed in the Table 1.2.

Table 1.2 Examples of substances used as excipients in tablet formulation (Alderborn 2002; Kottke and Rudnic 2002).

\begin{tabular}{|l|l|}
\hline Diluents (or fillers) & Surfactants \\
Binders & Antiadherents \\
Disintegrants & Antioxidants \\
Lubricants & Preservatives \\
Glidants & Colorants \\
Sorbents & Flavouring agents \\
\hline
\end{tabular}

The behaviour of the hydrophilic polymer in the presence of these substances in the formulation is important because the physicochemical characteristics of the co-excipients may alter the release mechanisms of the drug and the release rate from the matrix. For instance, an increase in the amount of diluents in matrix formulations generally involves an increase in the drug-release rate (Lotfipour, Nokhodchi et al. 2004). This increase in the release rate has been demonstrated in the case of matrices formulated with Carbopol $^{\mathrm{TM}}$ as the retardant agent, ibuprofen and three diluents, i.e., lactose, microcrystalline cellulose and starch (each diluent was tested separately) (Khan and Jiabi 1998; Majid Khan and Zhu 1999). Studies on the 
influence of the presence of magnesium stearate, a well-known lubricant, in the formulation of hydrophilic matrices on the drug-release profiles have been contradictory (Lee, Ryu et al. 1999; Rekhi, Nellore et al. 1999).

The inclusion of various additives, ranging from sugars to salts and other polymers, in SR matrix systems can be employed to affect matrix swelling, erosion and/or solubility of the drug and, thus, to modulate release kinetics. Examples of this strategy include the use of soluble diluents such as lactose to serve as erosion promoters in combination with swelling matrix formers such as HPMC (Gao, Skoug et al. 1996).

The incorporation of certain excipients within the formulation so as to improve the solubility of a weak acid or a weak base drug is one of the main approaches that have been used to obtain pH-independent release (Internal addition) (Bassi and Kaur 2010). These $\mathrm{pH}$ modifiers alter the $\mathrm{pH}$ in the microenvironment, i.e., the $\mathrm{pH}$ of the saturated solution in the immediate vicinity surrounding drug particles (Siepe, Lueckel et al. 2006; Tran, Tran et al. 2008), and thus bring the $\mathrm{pH}$ inside the formulation to a value where drug solubility is higher. This increases the drug dissolution and release irrespective of the $\mathrm{pH}$ of the external dissolution medium. Examples of these excipients are organic acids such as adipic, fumaric, tartaric and succinic acid, which can be used to increase the solubility of weak bases by decreasing the $\mathrm{pH}$ of the microenvironment (Gabr 1992; Siepe, Lueckel et al. 2008), and basic excipients such as dicalcium phosphate (DCP), magnesium oxide $(\mathrm{MgO})$ and magnesium hydroxide $\left(\mathrm{Mg}(\mathrm{OH})_{2}\right)$, used to increase the solubility of weak acids by increasing the $\mathrm{pH}$ of the microenvironment (Doherty and York 1989).

The incorporation of surfactants, such as sodium dodecyl sulfate, sodium lauryl sulfate, cetyltrimethylammonium bromide and sorbitan monostearate has also been studied as a possible strategy to modify the release profile of drugs (Ford, Mitchell et al. 1991; Nokhodchi, Hassan-Zadeh et al. 2008). 


\subsection{Starch and starch derivatives for sustained drug-release}

\subsubsection{Starch}

Starch, a naturally occurring homopolysaccharide of $\alpha$-D-glucose, has been one of the most widely studied pharmaceutical excipients, partially because it is biodegradable and metabolized by the human body. Besides safe, starch is an abundant, cost-effective and renewable material (Kost and Shefer 1990). Biodegradable synthetic polymers have the disadvantage of a possible toxicity following absorption of the degraded products, whereas native starch and starch derivatives are a safer alternative. Therefore, unmodified and modified starches have been used as excipients for various purposes in tablet formulation such as diluents, binders, disintegrants (Biliaderis 1991). Modified starches have also been proposed as agents for oral CR (Herman, Remon et al. 1989,; Lenaerts, Dumoulin et al. 1991). These starch and starch-derived polymers are subject to biodegradation by $\alpha$-amylase enzymes naturally present in the gastrointestinal tract. Starch is relatively resistant to enzymes other than $\alpha$-amylase and amyloglucosidase (Newman, Mueller et al. 2006).

Starch is the major storage carbohydrate of all higher plants (Biliaderis 1991). Starch granules are mainly found in seeds, roots and tubers, as well as in stems, leaves, fruits and even pollen (Pérez and Bertoft 2010). It can be extracted from several economically important cereals such as wheat, corn, rye or rice. Thus, it has always been a major source of food energy, in the form of carbohydrates, for humans and animals.

Since a derivatized starch was the base material of the present study, it will be described at greater length in this section of this thesis.

\subsubsection{Starch constituents and chemical structure}

Starch appears in the form of granules that have a heterogeneous content. The granules occur in all shapes and sizes (spheres, ellipsoids, polygon, platelets and irregular tubules) and their diameters vary from submicrons to $100 \mu \mathrm{m}$ (Pérez and Bertoft 2010). 
Starch granules consist of two major components: amylose, the non-branched fraction (Figure 1.11), and amylopectin, the branched fraction (Figure 1.12). Amylose is primarily a linear polysaccharide with $\alpha-(1 \rightarrow 4)$-linked D-glucose units. Some amylose molecules, particularly those of large molecular weight may have up to ten or more branches (Susumu Hizukuri 1981). Most starches contain a mixture of linear and branched amyloses (Hizukuri, Takeda et al. 1981; Takeda, Maruta et al. 1992). Amylopectin is a highly branched molecule and is composed of hundreds of short $\alpha-(1 \rightarrow 4)$-linked D-glucose backbones, interlinked by $\alpha$ $(1 \rightarrow 6$ )-linkages (about 5-6\% of total branches) (Buléon, Colonna et al. 1998; Pérez and Bertoft 2010). Amylopectin is made up of three classes of glucose chains, A, B and C. The A chains bind in clusters only to B chains, $\mathrm{B}$ chains bind to other $\mathrm{B}$ chains or to a $\mathrm{C}$ chain, which has a reducing end and of which there is one per molecule (Wang, Bogracheva et al. 1998). These two polysaccharides constitute $98-99 \%$ of the dry weight (Tester, Karkalas et al. 2004).

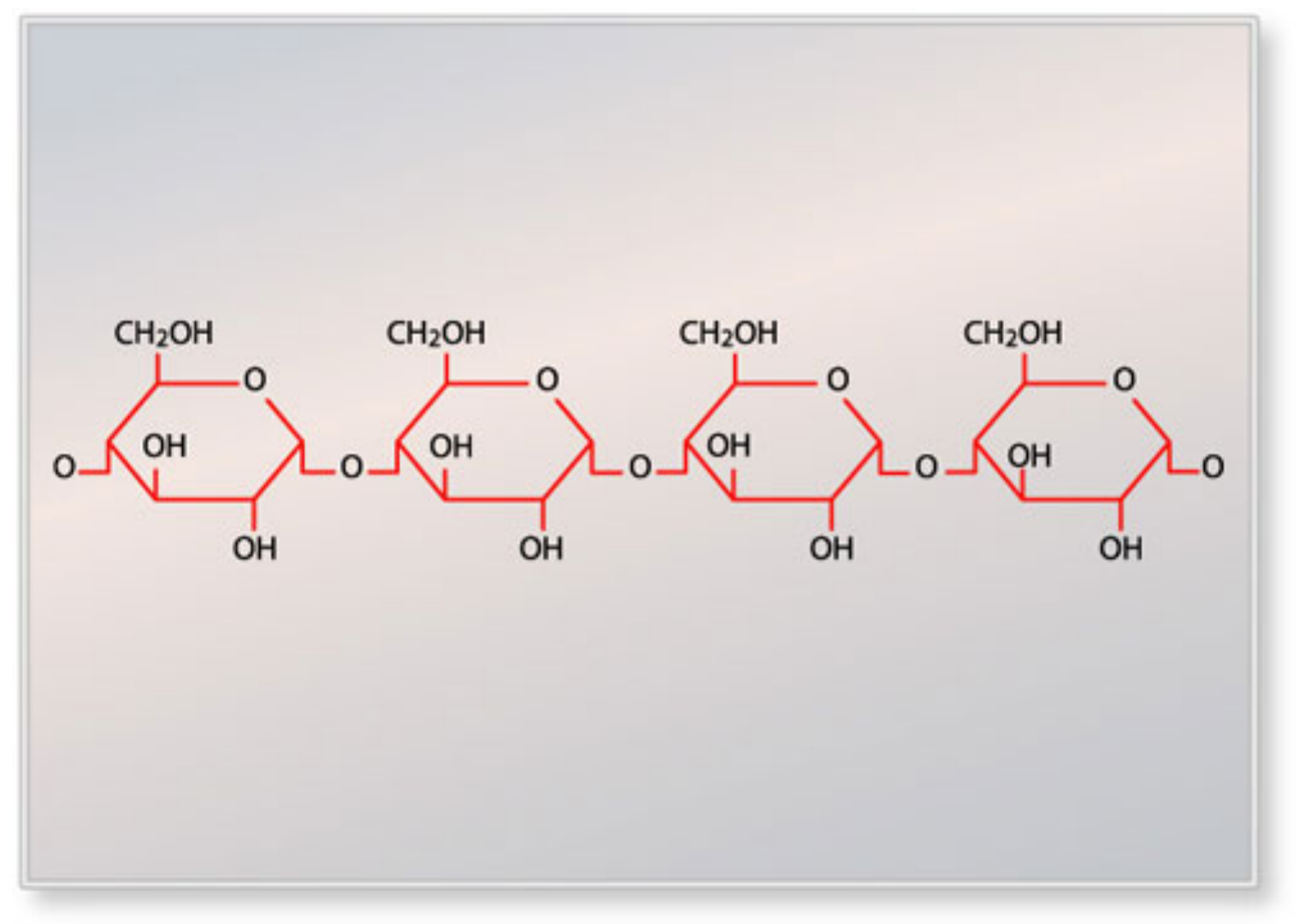

Figure 1.11 Schematic representation of the linear amylose macromolecule with its $\alpha-(1 \rightarrow 4)-$ linked D-glucose units. 


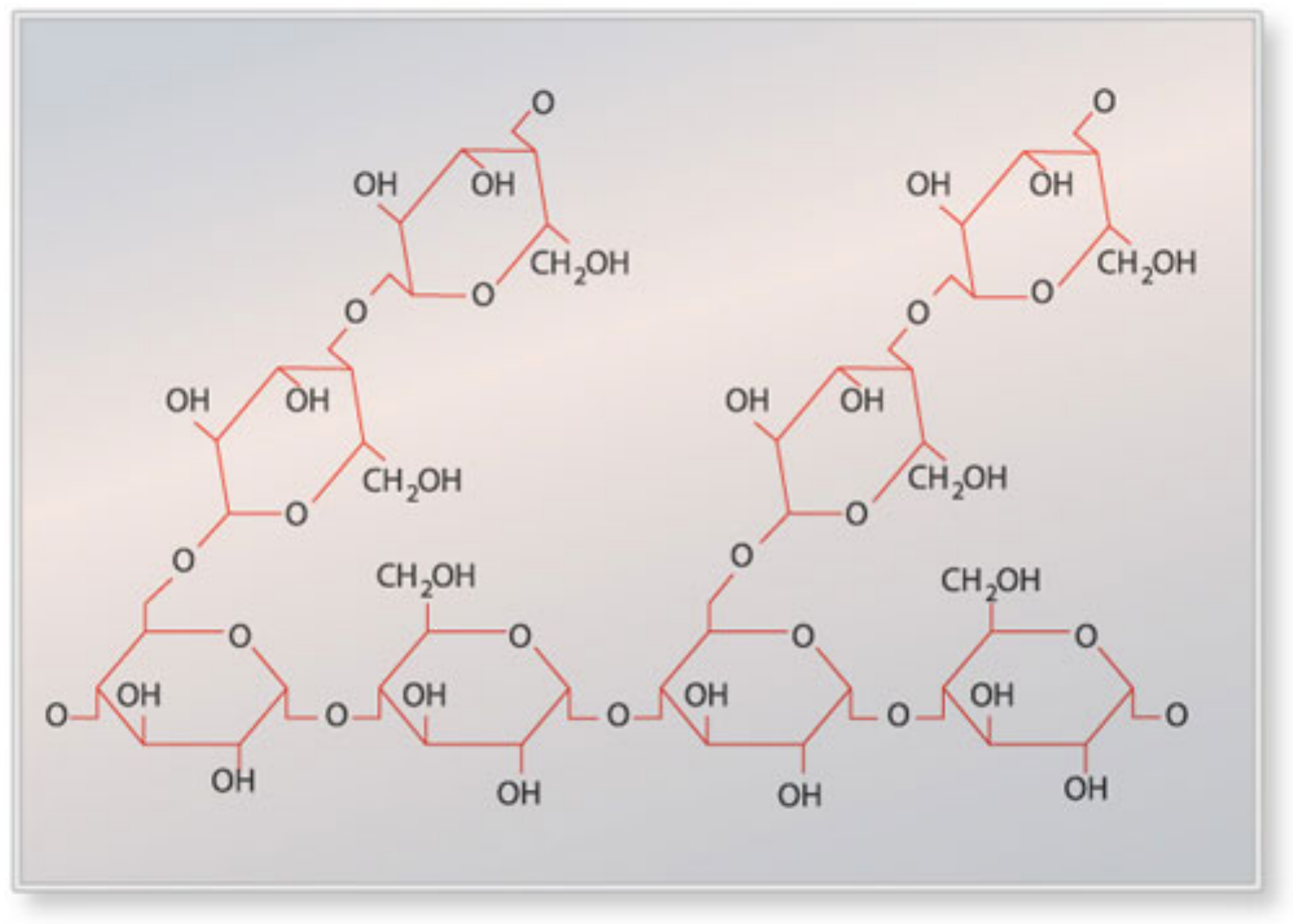

Figure 1.12 Schematic representation of the branched amylopectin macromolecule with its $\alpha$ $(1 \rightarrow 4)$-linked D-glucose backbones, interlinked by $\alpha-(1 \rightarrow 6)$-linkages.

Besides the two major polysaccharides, starch granules also contain minor components such as lipids, proteins, and phosphorus, which content varies according to the botanical origin of the starch, just as the main components (Pérez and Bertoft 2010). Normal starch, i.e., nongenetically modified, such as normal maize, rice, wheat and potato, contains about $70-80 \%$ amylopectin and 20-30\% amylose. However, it is possible to obtain starches with low-amylose content, known as waxy starches. Waxy starches contain less than 15\% amylose (Tester, Karkalas et al. 2004), or even less than 1\% in the case of waxy maize starch (Pérez and Bertoft 2010). Contrariwise, it also possible to produce high-amylose starch, which contains up to 80\% amylose (Pérez and Bertoft 2010). Typical molecular weights of extracted amylose are in the range of $10^{5}$ to $10^{6} \mathrm{~g} / \mathrm{mol}$. Amylopectin is one of the largest biopolymers known and its multiple branched structure has a molecular weight of about $10^{7}$ to $10^{9} \mathrm{~g} / \mathrm{mol}$, mostly in excess of $10^{8} \mathrm{~g} / \mathrm{mol}$ (Buléon, Colonna et al. 1998; Parker and Ring 2001). 


\subsubsection{Starch crystallinity and polymorphic types}

Native starches are biosynthesized as semi-crystalline granules with different polymorphic forms and degrees of crystallinity (Buléon, Colonna et al. 1998). These granules are complex structures consisting of crystalline and amorphous areas (Wang, Bogracheva et al. 1998). The crystallinity varies from 15 to $45 \%$ depending on the source of starch (Zobel 1988).

It is a common standpoint that short chains in the amylopectin molecule are organized into double helices, some of which then form crystalline lamellae (or crystallites) (French 1984; Blanshard 1987). The remaining double helices and the crystallites form the ordered part of the semi-crystalline starch granule, the remaining part being called the disordered or amorphous part (Wang, Bogracheva et al. 1998). The amorphous part of the starch granule is believed to consist of amylose and longer chains from the amylopectin (French 1984). Most starch granules are made up of alternating amorphous and semi-crystalline layers. These structures are termed 'growth rings' (French 1984; Pérez and Bertoft 2010). The semicrystalline layers are in turn constituted by periodic crystalline and amorphous lamellae (Pérez and Bertoft 2010).

In native starches, the double helices forming the lamellae are packed in different polymorphic types. Generally, most cereal starches give the so-called A-type X-ray diffraction pattern. Some starches of tubers (such as potato and lesser yam), rhizomes (e.g. canna) and cereal starches rich in amylose yield the B-pattern. C-type starches are found typically in the seeds of grain legumes, for example, peas (Wang, Bogracheva et al. 1998; Pérez and Bertoft 2010), and their diffraction patterns seem to represent a combination of A- and B-type polymorphs (Gernat, Radosta et al. 1990; Cairns, Bogracheva et al. 1997). The double helices in both A- and B-type are left handed and parallel stranded (Pérez and Bertoft 2010). These two types of polymorphs differ in their geometry, the packing density of their double helices and in the amount of water within the crystal structure, being the B-type a more highly hydrated and open structure (Figure 1.13) (Wang, Bogracheva et al. 1998; Parker and Ring 2001). 


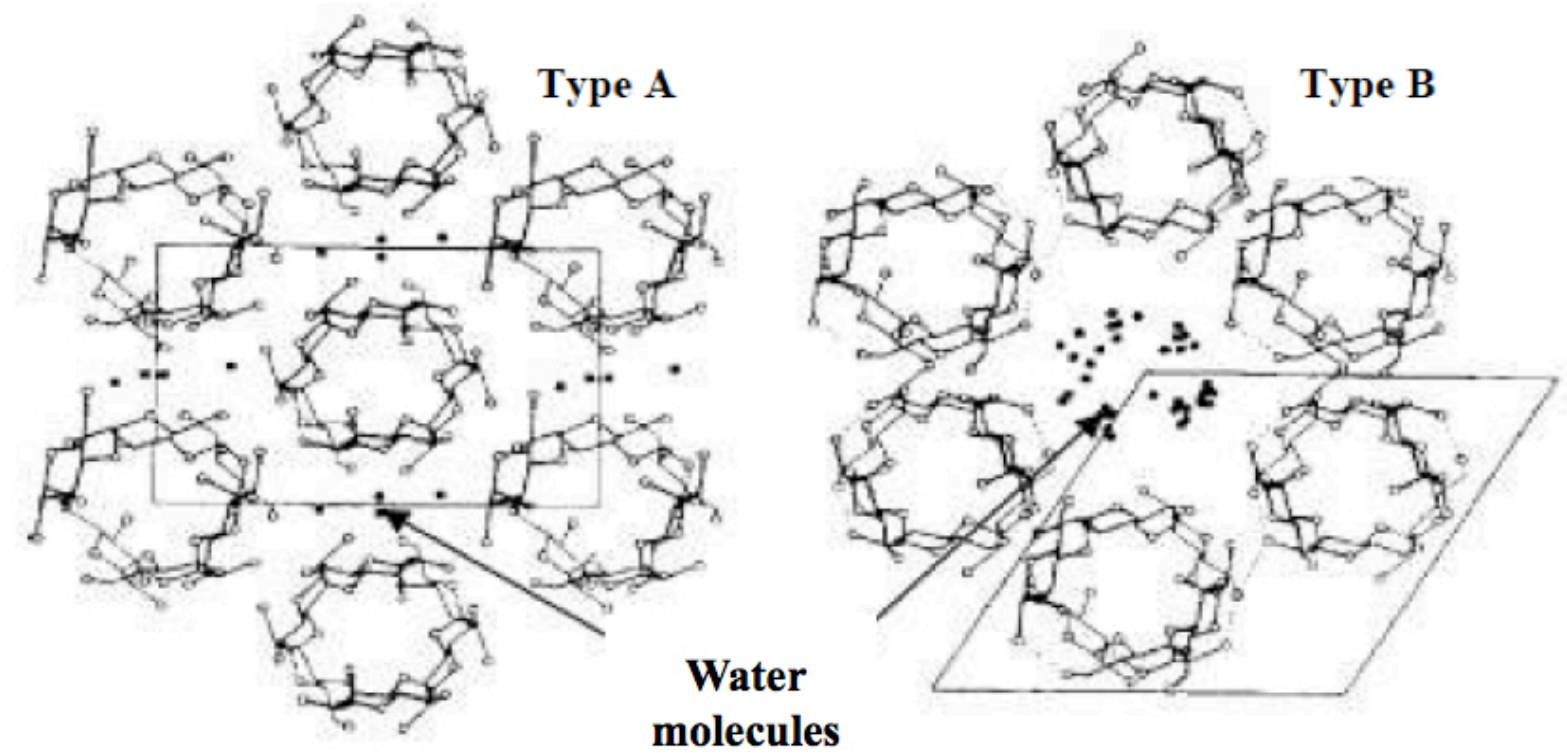

Figure 1.13 Comparison of units cells and helix packing in A- and B-amylose. Reproduced from (Hsien-Chih and Sarko 1978) with permission of Elsevier Ltd.

\subsubsection{Amylose as simple helices}

Single amylose chains have a unique ability to form complexes with a variety of complexing agents. Indeed, when amylose is precipitated from aqueous solution with suitable complexing agents such as dimethyl sulfoxide (DMSO) (Germino and Valletta 1964), acetone (Buléon, Delage et al. 1990) or iodine (Bluhm and Zugenmaier 1981), it crystallizes as "V" amylose complexes, which are left-handed amylose single helices and display the V-type diffraction pattern (from the German 'Verkleisterter stärke', meaning gelatinized starch, because it was first believed that the pattern represented pasted or gelled starch) (Pérez and Bertoft 2010). But, the most studied V-amylose complex is the form known as Vh (hydrated), which is normally induced by the presence of linear molecules such as linear alcohols (Buleon, Duprat et al. 1984; Whittam, Orford et al. 1989; Buléon, Delage et al. 1990; Brisson, Chanzy et al. 1991; Helbert and Chanzy 1994) or fatty acids (Godet, Buléon et al. 1993). 


\subsubsection{Starch behaviour in aqueous solution}

Native starches are insoluble in cold water, only swelling slightly (10-20\%), owing to penetration of water into the amorphous regions of the granule. However, this swelling is reversible upon drying. Contrarily, when starch is subject to gradual heating in aqueous medium, a point is reached where granule swelling becomes irreversible and structural order disappears, leading to loss of its crystalline properties. As the granules continue to expand, amylose leaches out into the aqueous intergranular phase. These processes result in substantial increase in viscosity (Biliaderis 1991). Overall, the disruption of granular structure, swelling and hydration, and solubilization of starch molecules are collectively described by the term gelatinization (Atwell, Hood et al. 1988).

Although the phenomenon of gelatinization is irreversible, starch gels are nonequilibrium states and, consequently, undergo structure transformation, i.e., further chain aggregation and recrystallization, during cooling or prolonged storage of the paste. This process is accompanied by gradual increases in rigidity and phase separation between polymer and solvent, and is described by the term retrogradation (Biliaderis 1991). NMR and X-ray diffraction experiments show that amylose precipitated from dilute aqueous solution contain only B-type aggregated double helices (Gidley 1989).

\subsubsection{Starch applications}

Starches and modified starches are widely and safely used, with the approval by the Food and Drug Administration (FDA), in the food industry as thickeners, enhancers of organoleptic properties and texture modifiers, and in the pharmaceutical industry as fillers, binders, disintegrants and glidants (Biliaderis 1991; Newman, Mueller et al. 2006), because, among of its other properties, starch is readily available, inexpensive, white, and inert, showing compatibility with numerous drugs.

The function of starch may depend on how it is incorporated into the formulation. Starch will function as a disintegrant when it is added in the dry state prior to adding a lubricant. It may exhibit both binding and disintegrating properties when it is incorporated 
either as a paste or dry before granulation with other agents (Newman, Mueller et al. 2006). It has been reported that starches deform mainly by plastic flow during compression, depending this phenomenon on the particle size, size distribution, and particle shape (McKenna and McCafferty 1982). A few mechanisms for the disintegration properties of starch have been proposed. The most common explanation is that disintegration is caused by the swelling of starch granules when exposed to water, and it has been proposed that amylose is the component responsible for the disintegration properties of starch due to swelling. A second theory suggests that the disintegration action of starch in tablets is due to capillarity. A third proposed mechanism is based on the particle-particle repulsion forces between the tablet constituents when in contact with water and the hydrophilic nature of starch (Guyot-Hermann and Ringard 1981).

\subsubsection{Modified starches}

In recent years, among polysaccharides, starch has received utmost attention because of its usefulness in different industrial products in modified form. Starch can be subjected to various physical (i.e. gelatinization) (Svensson and Eliasson 1995), chemical (Fang, Fowler et al. 2004), and enzymatic (Rajan, Prasad et al. 2006) modifications, or a combination thereof. These modifications are employed to create new starch products with specific or improved properties, such as control over drug-release. Indeed, native starches are not suitable for SR drug delivery systems because of their poor flow and compressibility and, especially, their fast release properties, which are a result of substantial swelling in aqueous media along with rapid enzymatic degradation by $\alpha$-amylase in physiological fluids (Kost and Shefer 1990; Dumoulin, Cartilier et al. 1999).

The main purpose of physical modification of starch is to obtain a completely or partially pregelatinized starch through heating in aqueous solution, followed by a rapid thermal drying (van Aerde and Remon 1988; Herman, Remon et al. 1989,). This modified starch, contrary to native starch, is soluble in cold water and is capable of forming a gel barrier. 
A number of pharmaceutical applications have been reported for physically modified starches. The most common use is as binder-disintegrants in immediate release tablet formulations. Some examples are a binder-disintegrant constituted of starches physically modified by compaction (Short and Verbanac 1971) and modified starches which demonstrated low swelling in cold water and are suitable for use as disintegrants in compressed tablets (Trubiano 1983). Moreover, physically modified starches have been tested as an excipient for SR (Nakano, Nakazono et al. 1987; van Aerde and Remon 1988; Herman and Remon 1989; Herman, Remon et al. 1989,; Herman and Remon 1990; A. Odeku and M. Picker-Freyer 2010). However, the results vary according to the authors, the types of drying methods applied and the origin of the starch. For example, studies (Herman and Remon 1989; Herman, Remon et al. 1989,; Herman and Remon 1990) on different types of pregelatinized starches found that pregelatinized starches containing a low amount of amylose $(25 \%$ and lower) could produce a strong enough gel layer to ensure a sustained drug-release in vitro, but their matrices were not able to preserve their integrity when administered in vivo. These authors attributed a negative role to amylose present in thermally modified starches used in sustained drug-release tablets.

Starches can undergo many reactions characteristic of alcohols because of the many hydroxyl groups present in their main constituents, amylose and amylopectin. Oxidation, ethoxylation, esterification, etherification and carboxymethylation are some of the chemical modifications commonly deployed to prepare starch derivatives. The properties of the resulting starch derivatives depend on many factors, such as plant source, prior treatment (acid-catalyzed hydrolysis or dextrinization), amylose/amylopectin ratio or content, molecular weight distribution or degree of polymerization, type of derivative (ester, ether, oxidized), nature of the substituted group, and physical form (granular, pregelatinized) (Wurzburg 1972; French 1984). Starch, a traditional disintegrant has been replaced extensively by a new class of disintegrants, commonly known as "super-disintegrants." An example of such superdisintegrant is sodium starch glycolate. It is manufactured by chemical modification of starch, i.e., carboxymethylation to enhance hydrophilicity and cross-linking to reduce the solubility of the carboxymethylated starch, thereby preventing the formation of a gel (Shah and Augsburger 2002). The starch used to produce this derivative contains a low content in amylose. 
Enzymatic modification usually corresponds to degradation by enzymes of starch chains. Depending on the enzyme used, specific cleavage of the link $\alpha(1-6)$ or of the link $\alpha(1-$ 4) will be observed. Disentanglement of chains results in a decrease in viscosity of the starch solution. The use of enzymatically hydrolyzed starches as excipients for sustained drug-release was studied by Te Wierik et al. (Te Wierik, Eissens et al. 1993; Te Wierik, Bergsma et al. 1996; Te Wierik, Eissens et al. 1997). These derivatives are presented as an alternative to conventional pregelatinized starches. A combination of physical modification with enzymatic modification was also used to produce SR excipients (Te Wierik, Eissens et al. 1997). The same authors studied the potential of debranched starches as suitable candidates for the application as filler-binder in tablets for direct compression (Te Wierik, Van der Veen et al. 1993; Te Wierik, Eissens et al. 1994; Te Wierik, Bergsma et al. 1996).

Over the last two decades, the chemical modification of high-amylose starch has been used to produce modified starches which posses interesting properties as excipients for sustained drug-release. One of main advantages of using high-amylose starch derivatives for the preparation of hydrophilic matrix tablets is related to the limited swelling and the high strength of the gels formed (Jane and Chen 1992 ). Two main chemical modifications have been used: cross-linking and substitution. Starches modified using these two methods will be described in the following sections.

\subsubsection{Cross-linked amylose}

Cross-linked amylose, commercialized as Contramid ${ }^{\circledR}$, was introduced in the early 1990s as an excipient for monolithic dosage forms able to control drug-release over an extended period of time, ranging between 18 and 24 hours (Lenaerts, Dumoulin et al. 1991). This semi-synthetic polymer is produced by the reaction of starch with high-amylose content with a cross-linking agent in an alkaline medium, forming a three dimensional structure. The agent usually used is epichlorohydrin (Lenaerts, Dumoulin et al. 1991; Moussa and Cartilier 1996; Moussa and Cartilier 1997; Dumoulin, Alex et al. 1998; Lenaerts, Moussa et al. 1998; Moussa, Lenaerts et al. 1998; Dumoulin, Cartilier et al. 1999), though trisodium trimetaphosphate, adipic-acetic anhydride and phosphorus oxychloride have also been 
mentioned (Dumoulin, Carriere et al. 1998).

Different degrees of cross-linking can be obtained by varying the ratio of epichlorohydrin to amylose in the reaction vessel. It was also found that water absorption, degree of swelling and drug-release rate from cross-linked amylose tablets increase with increasing cross-linking degree of the polymer (Lenaerts, Dumoulin et al. 1991; Moussa and Cartilier 1996). At high degrees of cross-linking, amylose is used as a tablet binder and/or disintegrant (Cartilier, Mateescu et al. 1997). However, effective control of the release of 1224 hours can be obtained for moderate degrees of cross-linking of the amylose (Lenaerts, Dumoulin et al. 1991).

It was demonstrated that in the dry state, there was a predominance of the V-type single helix arrangement of amylose irrespective of the cross-linking degree. However, upon hydration, the polymer with the lower cross-linking degree showed a transition from the $\mathrm{V}$ to the B-type double helix arrangement whereas the polymer with the higher cross-linking degree remains largely in the $\mathrm{V}$ form. The $\mathrm{V}$ to $\mathrm{B}$ transition generated a huge three-dimensional network of both chemical and physical links and a relatively tighter network than at a higher cross-linking degree. It was hypothesized that at higher cross-linking degrees, the ability of amorphous amylose chains to rearrange in double helices is probably hindered by the increased density in cross-links and hence a reduced mobility of the amylose chains. Therefore, the lower water uptake and drug-release rates with decreasing cross-linking degree were attributed to the tighter network obtained with a lower cross-linking degree (Dumoulin, Alex et al. 1998; Dumoulin, Carriere et al. 1998; Lenaerts, Moussa et al. 1998). Moreover, cross-linking as well as spray drying technique usually applied to dry the polymer, are believed to have a negative influence on the development of double helices and, thus, of crystalline organization (Guilbault and Mercier 1988).

Although Contramid ${ }^{\circledR}$ forms a gel membrane at the surface of the tablets within the first minutes of hydration (Moussa and Cartilier 1996; Lenaerts, Moussa et al. 1998), this gel layer thickens very slowly over time, giving the hydrated matrices of Contramid ${ }^{\circledR}$ a reservoirtype structure. The control of the release has been attributed only to diffusion of dissolved drug through the membrane (Moussa and Cartilier 1996). Therefore, an optimum level of chemical cross-linking ensures the integrity of the swollen tablet whose sponge-like structure enclosed by the membranous surface is responsible for SR (Le Bail, Morin et al. 1999). 


\subsubsection{Substituted amylose as a sustained drug-release agent in matrix systems}

Substituted amylose was introduced in 1999 as a pharmaceutical excipient for sustained drug-release tablets prepared by direct compression (Cartilier, Moussa et al. 1999; Chebli, Moussa et al. 1999). The SR properties of this starch derivative are a result of a substitution reaction by etherification of hydroxyl groups available in the chains of highamylose starch (containing $70 \%$ or $60 \%$ of amylose).

Several substitution agents have been proposed and most of the resulting excipients showed ability to slow down the release of drug. This family of starch derivatives is referred to as SA, R- $n$ where SA refers to Substituted Amylose, $\mathrm{R}$ is a code defining the substituent used and $n$ is the degree of substitution (DS), expressed as the ratio of moles of substituent per kilogram of amylose (Cartilier, Moussa et al. 1999; Chebli, Moussa et al. 1999).

This SR vehicle presents advantages at an economic level, since the production of matrix tablets with this new excipients is simple and easy, consisting of dry mixing of drug and SA polymers followed by compression, which is the easiest way to manufacture an oral dosage form.

\subsubsection{Synthesis at laboratory scale and general characteristics}

The process to synthesize substituted amylose at laboratory scale can be summarized as follows (Cartilier, Moussa et al. 1999; Chebli, Moussa et al. 1999):

1) Reaction between high-amylose corn starch and a substituting agent in alkaline media $(\mathrm{NaOH}, 1 \mathrm{~N})$ at $50^{\circ} \mathrm{C}$, in a Hobart planetary mixer at its slowest speed. The alkaline $\mathrm{pH}$ assures the activation of the reactive sites (positioned at C-6, C-2 and C-3) of the amylose chains. Different DS can be obtained by varying the substituent/amylose ratio (mole of substituent $/ \mathrm{kg}$ of amylose).

2) Neutralization of the well-mixed mass by adding, first, distilled water (heated to $50^{\circ} \mathrm{C}$ ), followed by the necessary volume of acetic anhydride to obtain a $\mathrm{pH}$ of 7.0. The homogenization was continued for an additional time at the same speed. 
3) Filtration of the resultant gel through a Büchner funnel, and its dehydration by successive washings with water and acetone or ethanol to recover the excipient in the form of a nearly dry powder. The powder product was exposed overnight to air at room temperature, which was sufficient to produce a powder ready to be compressed.
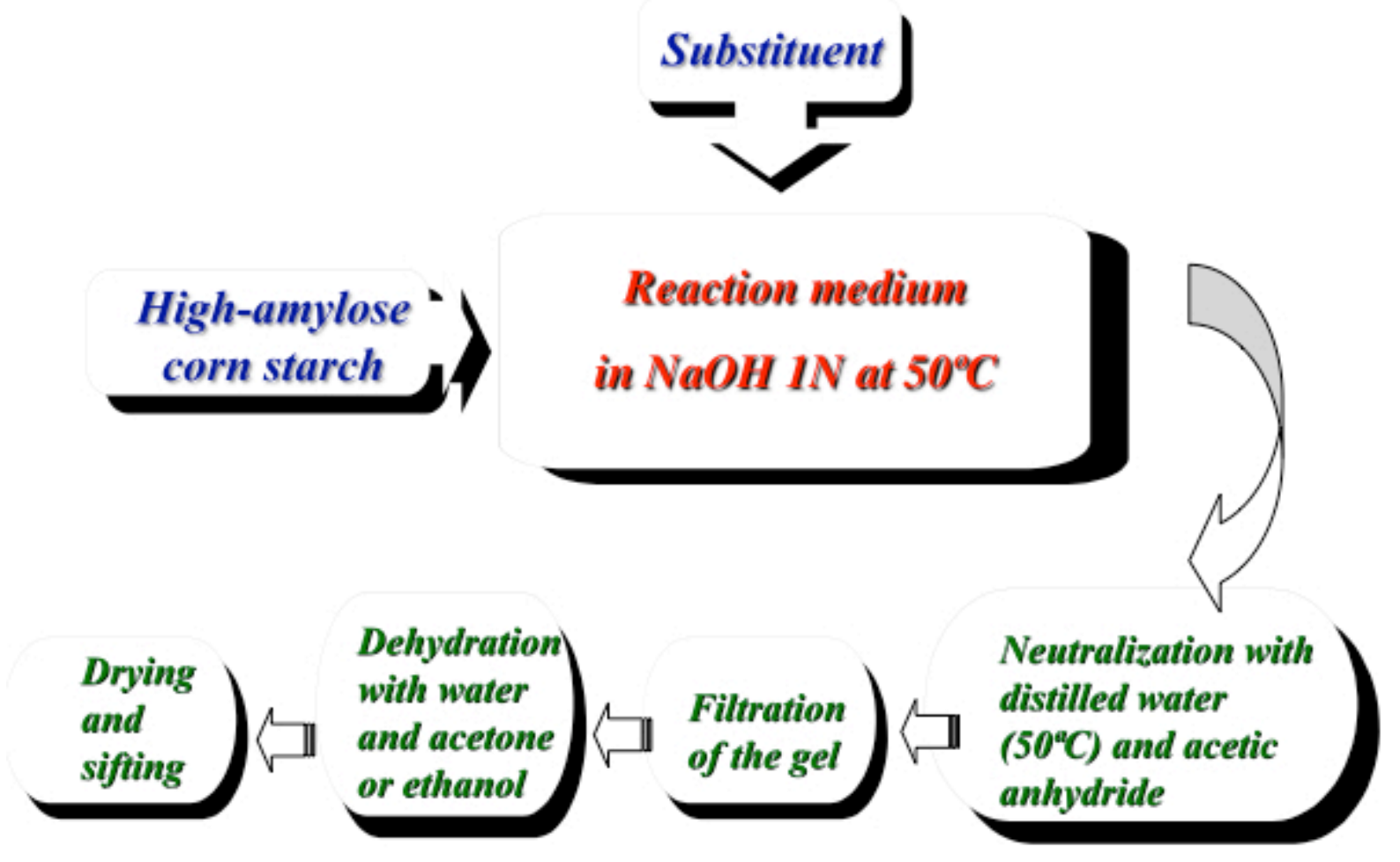

Figure 1.14 General representation of the synthesis of SA polymers at laboratory scale.

Since amylose contain reactive hydroxyl groups, being the one positioned on C-6 the most reactive, followed by hydroxyl groups on $\mathrm{C}-3$ and finally $\mathrm{C}-2$, it is possible to use a substituting agent and chemically modify these hydroxyl groups by an etherification process, resulting in substituted amylose (SA). A range of substituents such as 1,2-epoxypropanol (or glycidol = G), 1,2-epoxybutane, 1,2-epoxydecane and 1-chlorobutane, were investigated. However, the SA,G- $n$ polymers showed the most interesting properties in terms of good 
compression behaviour and sustained drug-release (Cartilier, Moussa et al. 1999; Chebli, Moussa et al. 1999).

Sustained drug-release matrix systems based on SA,G- $n$ technology presented large ranges for drug loading, drug solubility and tablet weight (TW) (Cartilier, Moussa et al. 1999; Chebli and Cartilier 2000). In vitro dissolution release times of $95 \%$ of the drug ranged from 9 to 20 hours for all DSs studied for $400-\mathrm{mg}$ matrices containing $10 \%$ of acetaminophen (Cartilier, Moussa et al. 1999; Chebli, Moussa et al. 1999). Moreover, DSs ranging from 0.4 to 3.4 had no significant effect on the release of acetaminophen but exerted a significant influence on the water uptake of SA,G- $n$ polymers, i.e., increasing the DS resulted in increased in hydrophilicity. However, beyond these values, the release properties decreased because of excessive water absorption, which led to the erosion of the system (Cartilier, Moussa et al. 1999; Chebli, Moussa et al. 1999). In contrast to pregelatinized starches known for their poor binding properties (Hancock, Carlson et al. 2001; Rahmouni, Lenaerts et al. 2002), the tablets produced with SA,G- $n$ polymers presented very high crushing strength values, comparable to those of microcrystalline cellulose tablets, a reference among binders/fillers (Cartilier, Moussa et al. 1999; Chebli, Moussa et al. 1999). In addition, a study showed no significant influence of CF ranging from 0.5 to 5.0 tons $/ \mathrm{cm}^{2}$ on the release properties of SA,G- $n$ polymers, with a DS greater than 1.5 (Chebli and Cartilier 2000). These features help ensure high reproducibility of batch performance and versatility despite possible variations during industrial production. It was also demonstrated that the importance of the relaxation of chains and erosion in the mechanism of drug-release increases with the DS, being diffusion the dominant drug transport mechanism for low DSs (Chebli, Moussa et al. 1999). Finally, a study on the release of rhenium (VII) oxide from SA,G- $n$ matrix tablets in the presence or the absence of $\alpha$-amylase enzymes revealed only a slightly faster SR of the drug from SA,G-2.7 matrix systems in the presence of the $\alpha$-amylase enzyme in the dissolution medium and no significant influence on the release properties when DS was increased to 4.0, showing that increasing the DS made the matrix less accessible to the enzymes (Chebli, Cartilier et al. 2001). This finding confirmed the value of this polymer for SR applications, since systems based on starch are normally susceptible to biodegradation by $\alpha$-amylase. 
1.3.3.2 Performance of amylose substituted by 1,2-epoxypropanol (SA,G-2.7)

Of all SA, G- $n$ polymers, SA,G-2.7 was the one which demonstrated the best performance as an excipient for sustained drug-release systems. SA,G-2.7 polymeric matrices obtained by direct compression allowed nearly constant drug-release (Figure 1.15) (Cartilier, Moussa et al. 1999; Chebli, Moussa et al. 1999; Chebli and Cartilier 2000). Such a release profile is unusual for a hydrophilic matrix system where a Fickian release, i.e. a first-order kinetics, is expected. Moreover, a study revealed that release time was directly proportional to TW for tablets containing 10\% of acetaminophen (Chebli and Cartilier 2000), showing that TW can be used as a formulation tool, independently of CF. It was also demonstrated that SA,G-2.7 matrices could be employed for a wide range of model drugs, independently of their nature, solubility, and concentration (Chebli and Cartilier 2000). Indeed, their release properties were maintained for both slightly soluble drugs (theophylline) and freely soluble drugs (chlorpheniramine maleate). Ninety percent of chlorpheniramine maleate was released in 11 hours from 500-mg SA,G-2.7 matrices with $10 \%$ of drug, whilst concentrations of theophylline ranging from 3 to $50 \%$ had released times between 8 and 18 hours from $600-\mathrm{mg}$ tablets, with a maximum obtained with a concentration of 10\% (Chebli and Cartilier 2000).

The most important aspect of the release mechanism from tablets constituted by SA,G2.7 is believed to be related to the ability of this high-amylose polymer to hydrate and form a rigid and long-lasting gel on the surface of the tablets in response to water penetration. This gel layer acts like a barrier against the fast release of drugs whilst controlling at the same time the penetration of aqueous medium and the diffusion of drug. 


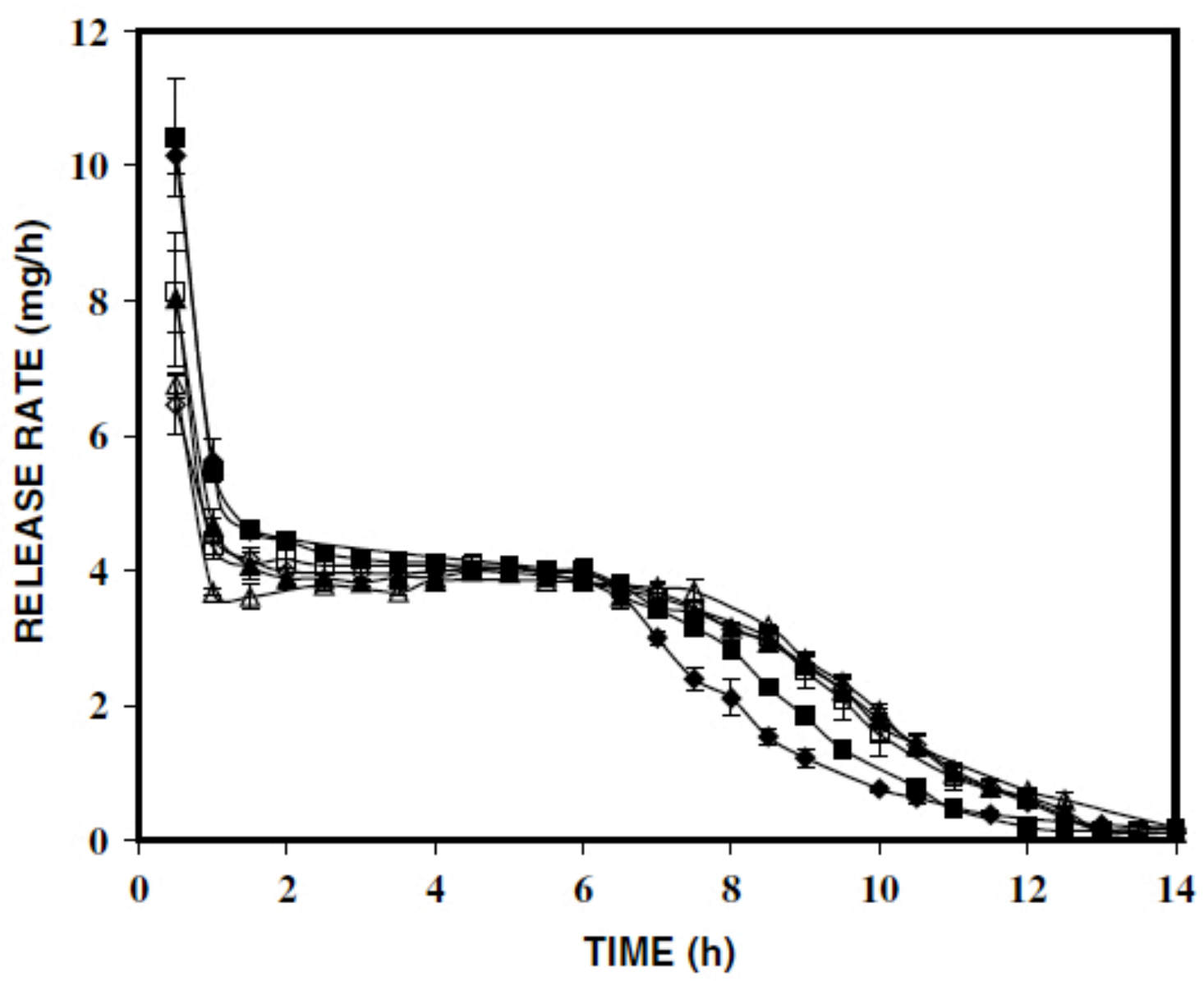

Figure 1.15 Release rate of acetaminophen from SA,G-2.7 matrices (compressed at CF between 0.5 and 5.0 tons) as a function of dissolution time, showing a nearly constant drugrelease from approximately $1 \mathrm{~h}$ to $6 \mathrm{~h}$, and three different regions for the drug-release rates. Figure reproduced from (Moghadam, Wang et al. 2007) with permission of Elsevier Ltd.

The drug-release rates from SA,G-2.7 matrix tablets in function of time showed three clearly distinguishable regions (Figure 1.15) (Chebli and Cartilier 2000; Moghadam, Wang et al. 2007). The first region presented high release rates for all CF, although the initial release rate seemed to depend on the CF. This phenomenon, known as "burst effect", is characteristic of hydrophilic matrix systems and is due to the drug dissolution occurring at the surface of the tablet, before the polymeric gel membrane forms and hinders drug diffusion outside of the matrix (Huang and Brazel 2001). In addition, the burst effect is influenced by the tablet 
porosity, and thus by the CF applied. The second region, however, was not representative of a hydrophilic matrix system as it showed an almost constant release rate, maintained for a few hours and independent of the CF, while the third region showed a first-order-like decline of the release rate for all tablets. These two regions are more typical of a reservoir system, where the drug is released constantly through a membrane until the inner compartment no longer contains a drug-saturated solution. This hybrid behaviour, typical of a hydrophilic matrix and a reservoir system, can be explained by the sequence of events that follow the immersion of a SA,G-2.7 tablet in an aqueous medium (Chebli and Cartilier 2000; Moghadam, Wang et al. 2007): during the burst effect, the polymer at the surface of the tablet hydrates and the hydrated amylose chains associate through hydrogen bonds created between hydroxyl groups present on the glucose units (Biliaderis 1991) and form a rigid gel. This external gel membrane controls the penetration of aqueous medium towards the core of the matrix and the release of drug by diffusion. The slow medium penetration allows the maintenance of a drugsaturated solution in a core surrounded by a gel membrane that swells gradually, resulting in an almost constant drug-release until the internal hydration and the concentration of drug do not allow the existence of a drug-saturated solution in the interior of the matrix.

It was observed that for drug loadings lower than $30 \%$ of acetaminophen, the $90 \%$ release time increased as a function of drug loading (Moghadam, Wang et al. 2007). This behaviour was explained considering the continuous membrane-like structure created around the tablet and that augmenting the drug concentration resulted in an increase of the drug content in the core of the tablet, and thus in an increase of the $90 \%$ release time. However, in order for this phenomenon to happen, the structure of the external gel membrane has to be maintained intact. Thus, for drug loadings larger than $30 \%$, the physical erosion of the membrane led to an acceleration of drug-release and to a continuous decrease of the $90 \%$ release time as a function of the drug loading, which is the typical behaviour of hydrophilic matrices.

High-amylose content was referred to be an essential feature to obtain sustained drugrelease properties from SA,G-2.7 matrices and other starch derivatives (Cartilier, Moussa et al. 1999). This occurrence can be explained by the creation of a gel network due to the transition from an essentially amorphous character in the dry state into A or B crystalline form when the tablet is immersed in an aqueous environment. However, this phenomenon will only take 
place if a critical concentration of amylose is present in the starch material. Common starches, i.e. starches presenting high concentrations of amylopectin and low concentrations of amylose, do not form a rigid gel allowing drug-sustained release.

An evaluation of the combined effect of drug loading and TW on the SR properties of SA,G-2.7 showed that a minimum weight of $300 \mathrm{mg}$ was required to obtain drug-sustained release (Moghadam, Wang et al. 2007). Tablets with lower weights were too thin to be able to create an external membrane controlling drug diffusion, because the matrix was fully hydrated too quickly.

The excellent compression behaviour of this polymer was demonstrated by measurements of the crushing strengths and the thickness of tablets for different drug concentrations and CF (Chebli and Cartilier 2000; Moghadam, Wang et al. 2007). A study (Moghadam, Wang et al. 2007) revealed that the relationship between tablet thickness (TT) and TW was linear and independent of the CF applied, except for a slight deviation observed in the case of the lowest CF $\left(1.5\right.$ tons $\left./ \mathrm{cm}^{2}\right)$, and that the densification was the same for all $\mathrm{CF}$, indicating that an optimal particle rearrangement occurred and that some peculiar phenomenon took place, even at low compressions. Image analysis by scanning electron microscopy was used to observe the structure of SA,G-2.7 tablets. The core of these tablets was completely melted while the external layer went through densification, deformation and partial melting. This melting phenomenon, which is not observable amidst pharmaceutical excipients such as HPMC or pregelatinized starches, offered an explanation for the high crushing strength values observed for SA,G-2.7 tablets. In addition, a measure of the porosity of these tablets showed that the total porosity was very weak, since pores were present in a very low and narrow range, i.e., around $12 \mathrm{~nm}$, while HPMC tablets presented an important porosity with large pores. This porosity study confirmed that SA,G-2.7 tablets went through an important densification process. It was also found that the water uptake values were not affected by CF in the range 1.5-5.0 tons $/ \mathrm{cm}^{2}$ (Moghadam, Wang et al. 2007), which reinforces the theory of an absence of effect of tablet porosity on the drug-release rate after the burst-effect. 


\subsubsection{High-amylose sodium carboxymethyl starch}

Although SA,G- $n$ polymers presented good properties as sustained drug-release excipients, the reaction of high-amylose starch with sodium chloroacetate or chloroacetic acid (Figure 1.16) instead of nonionic substituents such as 1,2-epoxypropanol (or glycidol) was later proposed to obtain a new substituted amylose derivative (Cartilier, Ungur et al. 2005; Ungur, Yonis et al. 2005).

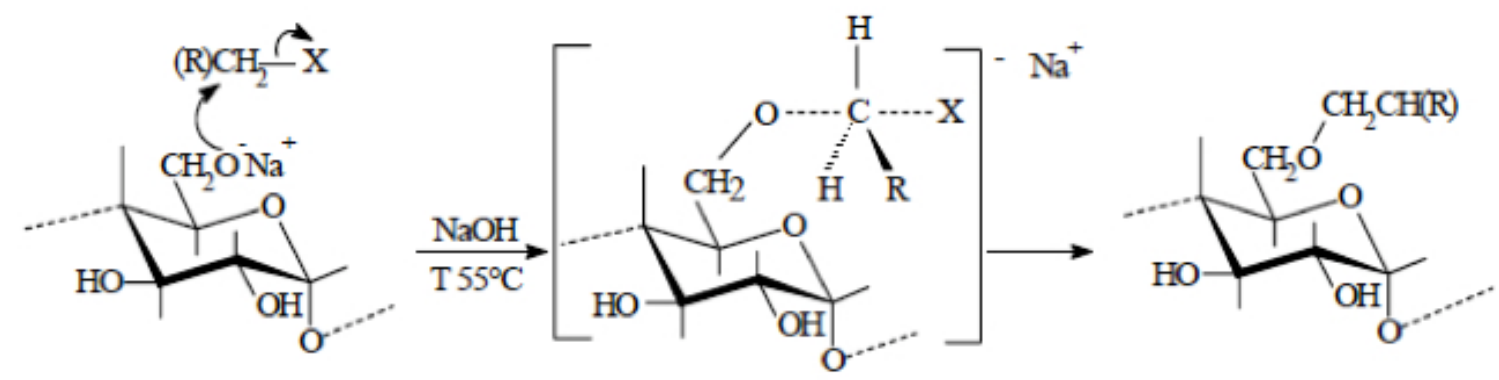

Figure 1.16 Reaction of substitution of amylose by sodium chloroacetate.

Sodium carboxymethyl starch produced from starch with low-amylose content and substituted with sodium chloroacetate has been used as a well-known disintegrant agent in IR tablets (Bolhuis, Van Kamp et al. 1986; Edge and Miller 2005) as well as in the food industry for decades, and has thereby proven its safety. Therefore, it is more readily acceptable by regulatory agencies such as the FDA. This high-amylose sodium carboxymethyl starch was first referred to as SA,CA- $n$, where "CA" stands for chloroacetate and $n$ the DS.

As the polymer is ionic, the tablets can be formulated with the addition of an electrolyte, which allows the integrity of the swollen matrices to be maintained when they are immersed in media undergoing $\mathrm{pH}$ changes simulating the $\mathrm{pH}$ environment along the gastrointestinal tract, while allowing controlled and sustained drug-release. This electrolyte can be another excipient, a drug or mixture thereof (Cartilier, Ungur et al. 2005; Nabais, Brouillet et al. 2007). 
1.3.4.1 High-amylose sodium carboxymethyl starch produced at laboratory scale

SA,CA- $n$ was first synthesized at laboratory scale following the same methodology used to prepare SA,G- $n$ polymers. SA,CA- $n$ polymers with DSs (also expressed as the number of moles of substituent added per kilogram of amylose) ranging from 1.55 to 2.7 were produced and the SA,CA- $n$ which presented the closest characteristics to SA,G-2.7 was selected. Scanning electron microscopy was used to compare the structures of the granules of both polymers. SA,CA- $n$ granules presented the same main characteristics, i.e., an amorphous character and a high porosity (Cartilier, Ungur et al. 2005).

Dissolution studies demonstrated SR of acetaminophen from tablets containing SA,CA- $n$ produced at laboratory scale independently of the DS, within the range of DSs used to produce these polymers. Indeed, DSs ranging from 1.55 to 2.7 did not significantly influence the drug-release profiles in spite of a significant influence on the absorption of water, which increased with the number of carboxyl groups. The drug-release rates of SA,CA$n$ were, however, slightly faster than for SA,G- $n$ and the release profiles less linear (Cartilier, Ungur et al. 2005).

Initially, for comparison with the SA,G- $n$ polymer, dissolution tests were carried out only in a phosphate buffer at $\mathrm{pH}$ 7.4. However, compared to glycidol, the new substituting agent confers an ionic nature to the SA,CA- $n$ polymer. This had to be taken into account when assessing the behaviour of this polymer, given that the $\mathrm{pH}$ gradient throughout the digestive system can have a significant influence on the behaviour of ionic polymers. For this reason, SA,CA- $n$ tablets started being tested in a $\mathrm{pH}$ gradient $(\mathrm{pH}=1.2$ and $\mathrm{pH}=7.4)$ in place of an unique phosphate buffer. When using a $\mathrm{pH}$ gradient, the same type of sustained drug-release profiles was observed (Cartilier, Ungur et al. 2005).

The release profiles from SA,CA-1.55 matrices with $10 \%$ of acetaminophen in a $\mathrm{pH}$ gradient bore the closest resemblance to the release profiles from SA,G-2.7 matrices. In addition, as the residence time in the stomach can vary, studies on the influence of the residence time in the acidic medium on the in vitro drug-release from SA,CA-1.55 matrices were performed. These studies showed no significant influence of residence times ranging from 0.5 to 2.0 hours on the release of acetaminophen and led to the selection of SA,CA-1.55 as the prototype SA,CA polymer produced at laboratory scale. Although cracks on the surface 
of the tablets produced with this polymer appeared during the dissolution tests, they did not significantly influence drug-release. In addition, the use of a $\mathrm{pH}$ gradient instead of a sole phosphate buffer delayed the development of cracks (Cartilier, Ungur et al. 2005).

The performances of this prototype allowed considering a transition to a pilot-scale development.

\subsubsection{High-amylose sodium carboxymethyl starch produced at pilot scale}

The first pilot scale batch was obtained from Roquette Frères S.A. (Lestrem, France) and consisted of a sodium salt of high-amylose carboxymethyl starch with a DS of 0.05 , thus referred to as SA,CA-0.05. It is important to point out that the expression of DS employed by the company in the fabrication of the polymer is different from the definition used to produce SA, G- $n$ and SA,CA- $n$ at laboratory scale. In this case, the DS was expressed as number of moles of substituent per number of moles of anhydroglucose (dry weight of starch divided by 162 , the molecular weight of anhydroglucose). For convenience of description, pilot scale SA,CA-0.05 was later renamed HASCA in this work, where "HAS" means high-amylose starch and "CA" defines chloroacetate. This term will be used hereafter.

Pilot scale HASCA was produced via an industrial process different from the laboratory process, using a drying method without organic solvents. However, this excipient was not suitable for tableting by direct compression and sustained drug-release. Therefore, the dry powder of pilot scale HASCA was dispersed in hot water, and then precipitated with ethanol using the laboratory process, as previously described, though the original process used acetone to precipitate SA polymers. The recovered dry powder possessed the required binding and SR properties (Cartilier, Ungur et al. 2005; Ungur, Yonis et al. 2005).

However, as it happened for lab scale SA,CA-1.55, the tablets produced only with the new polymer and the drug presented cracks on their surface. To solve this problem several associations with additives of different nature, i.e., gelling ionic or nonionic polymers, pregelatinized starch, dextrin, soluble and non-ionic diluents or insoluble diluents, were tested without success (Ungur, Cartilier et al. 2004; Cartilier, Ungur et al. 2005). Surprisingly, ionic soluble compounds, i.e., organic acids and organic bases of low molecular weight, buffering 
agents and electrolytes, were the substances that added to the tablet formulation led to the best results regarding the maintenance of the tablet integrity during the dissolution tests in a $\mathrm{pH}$ gradient. It was expected that these hydrophilic substances, and in particular the electrolytes, would exacerbate the occurrence of cracks by increasing significantly the penetration of water into the matrix and, as a consequence, the osmotic pressure inside the matrix. Instead, the addition of sodium chloride $(\mathrm{NaCl})$ helped maintain the integrity of the swollen matrix tablets without affecting the SR properties of HASCA (Cartilier, Ungur et al. 2005).

Like the SA,G- $n$ polymers, HASCA revealed to possess numerous qualities as a pharmaceutical excipient for SR of drugs (Ungur, Cartilier et al. 2004; Cartilier, Ungur et al. 2005). Despite their ionic nature, the SR properties of HASCA-based matrix tablets were not influenced by residence times in acidic medium ranging from 0.5 to 2.0 hours when the tablets were tested in a $\mathrm{pH}$ gradient. Besides, even in the presence of $\mathrm{NaCl}$, the release rate of acetaminophen from HASCA tablets was not significantly influenced by the CF applied or by the addition of magnesium stearate, a well-known table lubricant, to the formulation. HASCA tablets were capable of delaying the release of pseudoephedrine hydrochloride, an ionic and highly soluble drug, from tablets containing $60 \%$ of drug, when they were evaluated in a $\mathrm{pH}$ gradient, which support the excellent properties of this new excipient. The release time of pseudoephedrine hydrochloride increased with an increase in TW, but decreased with an increase in drug loading, indicating that both the TW and the drug concentration can be used to optimize a formulation containing HASCA as the SR excipient. Moreover, HASCA tablets are characterized by the same unusually high crushing strength values observed for SA,G- $n$.

The characteristics summarized above illustrate the qualities of high-amylose sodium carboxymethyl starch as a functional and performing hydrophilic excipient for the formulation of SR systems. However, the proposed manufacturing process required very high volumes of ethanol to precipitate and recover the final product, which represented a drawback for a change in scale from laboratory to industrial production because it limited the economic viability of this excipient. In order to prepare the scale-up for easier and more economical industrial production of HASCA, Brouillet et al. (Brouillet, Baylac et al. 2010) designed a process to transform by spray drying totally amorphous HASCA into an efficient sustained drug-release excipient for matrix tablets while decreasing ethanol quantities. The spray drying 
method has the advantage of being able to produce a fine powder while drying the solid. The same methodology was used to prepare the HASCA polymer used in the experiments of the present work and will be briefly described in the next section.

\subsubsection{Spray-dried high-amylose sodium carboxymethyl starch}

The process used in this work to produce spray-dried high-amylose sodium carboxymethyl starch (SD HASCA) with the required binding and sustained drug-release properties was previously developed by Brouillet et al. (Brouillet, Bataille et al. 2010) and included the following steps:

1) Dispersion of pilot-scale HASCA in a solution composed of water and at least one pharmaceutically acceptable organic solvent miscible with water and suitable for spray drying; In this case ethanol was the chosen organic solvent.

2) Heating of the dispersion obtained in the first step;

3) Addition of a second volume of the same organic solvent used in the first solution to the heated dispersion to decrease its viscosity to easily process the suspension through the spray dryer; and

4) Spray drying of the final dispersion in order to obtain a dry powder of HASCA comprising a major fraction of amorphous form and a minor fraction of crystalline Vh-form.

Because of the presence of its hydroxyl groups, amylose in aqueous solution forms a viscous gel through strong hydrogen bonding. The high viscosity of the dispersion of HASCA will difficult its process through the spray dryer. In order to limit the formation of a viscous starch gel a water/organic solution was used to disperse HASCA (Tijsen, Scherpenkate et al. 1999; Tijsen, Kolk et al. 2001; Tijsen, Voncken et al. 2001). The organic solvent chosen in this process was ethanol, because it is pharmaceutically acceptable, miscible with water and compatible with spray drying methods, and it has been tested before for this purpose (Sloan, Mehltretter et al. 1962; Thewlis and Albans 1969). It is believed that alcohol disrupts the amylose gel structure by bonding to hydroxyl groups on starch molecules. Unlike hydrogen bonding between adjacent amylose chains through water molecules, this binding is terminal 
and produces no connection between amylose molecules, reducing the apparent viscosity of the solution and resulting in amylose precipitation at high alcohol concentrations (McGrane, Mainwaring et al. 2004).

After optimization of the process, tablets produced with SD HASCA presented high crushing strengths. It was hypothesized that these high values were in part due to the decreased particle size of the powder obtained after spray drying. Second, the combination of water and ethanol may have a plasticizer effect, causing partial melting of the excipient and particle re-arrangement under compression (Brouillet, Baylac et al. 2010) This atypical melting process was demonstrated using scanning electron microscope and porosimetry in the case of SA,G-2.7 (Moghadam, Wang et al. 2007) as described in a previous section.

\subsection{Physiological factors influencing oral drug-absorption}

As previously described, the oral route of administration requires an absorption step before the drug reaches the systemic circulation. The factors affecting drug absorption depend on the physiology of the gastrointestinal tract and on the passage of the drug through the gastrointestinal membrane towards the blood circulatory system.

\subsubsection{Physiology of the gastrointestinal tract}

The gastrointestinal tract is a highly specialized region of the body, which includes organs and glands whose primary functions involve: 1) processes of secretion of fluids (secreted by the salivary glands, pancreas, liver and the gastrointestinal epithelial cells) composed of mainly digestive enzymes, ions, mucus and bile; 2) guiding food as it passes through and its digestion, which involves the breakdown of food into smaller components in order to permit the extraction and absorption of its nutrients into the bloodstream; 3) passing out remaining wastes for elimination. 
The gastrointestinal tract consists of a muscular tube that stretches from the mouth to the anus and consists of four main anatomical areas: the oesophagus, the stomach, the small intestine and the large intestine or colon (Figure 1.17) (Ashford 2002; Marieb and Hoehn 2010). The luminal surface of the tube is not smooth but very rough, thereby increasing the surface area for absorption. The different areas of the gastrointestinal tract present their own anatomy, physiology and environmental characteristics concerning, for example, the composition of gastrointestinal fluids, the type of secretion, the $\mathrm{pH}$ value, the nature of enzymes and the transit time (Table 1.3). These particularities define the role of each anatomical area in the digestive process. However, a certain structural similarity can be observed along the length of the wall of the gastrointestinal tract except for the mouth, consisting of four principal histological layers, which form the apical surface (lumen) of the gastrointestinal tract to the basolateral surface (bloodstream) are (Washington, Washington et al. 2001; Ashford 2002; Marieb and Hoehn 2010):

1. The mucosa, which is essentially composed of three layers. The first layer is the epithelium. The epithelium lines the gastrointestinal lumen and is implied in the absorption of nutrients and drugs, in the secretion of enzymes and mucus, and in the immune defence of the organism and protection of the tissues that lie beneath it. The epithelium lies on a layer of mainly connective tissue known as the lamina propria (Figure 1.18), which provides structural support and assures the blood supply and lymphatic drainage of the epithelium. The lamina propria contains many types of cells, such as lymphocytes, macrophages and plasma cells, and it is believed that it has an important role in preventing the entry of microorganisms and foreign substances. This layer is followed by the muscularis mucosa, a thin layer of smooth muscle that can change the local conformation of the mucosa and separates it from the submucosa.

2. The submucosa, which is a layer of connective tissue containing some secretory tissue. The submucosa is richly supplied with blood and lymphatic vessels. A network of nerve cells, known as the submucosal plexus (or "Meissner's plexus"), is also located in this layer;

3. The muscularis externa, which consists of an inner circular layer and an outer longitudinal layer of smooth muscle, being the outer layer thinner than the inner one. Contractions of these muscles provide the forces for movement of gastrointestinal contents; 
4. The serosa, also called serous membrane is a membrane formed by a wet thin layer of epithelial cells overlying some loose connective tissue.

The majority of the gastrointestinal epithelium is covered by a layer of mucus, a viscoelastic translucent gel composed mostly of water $(\sim 95 \%)$ and large glycoproteins called mucins, which are responsible for its physical and functional properties. Mucus is secreted throughout the gastrointestinal tract and acts as a mechanical barrier and a protective layer against the proteolytic action of digestive enzymes (Khanvilkar, Donovan et al. 2001; Ashford 2002).

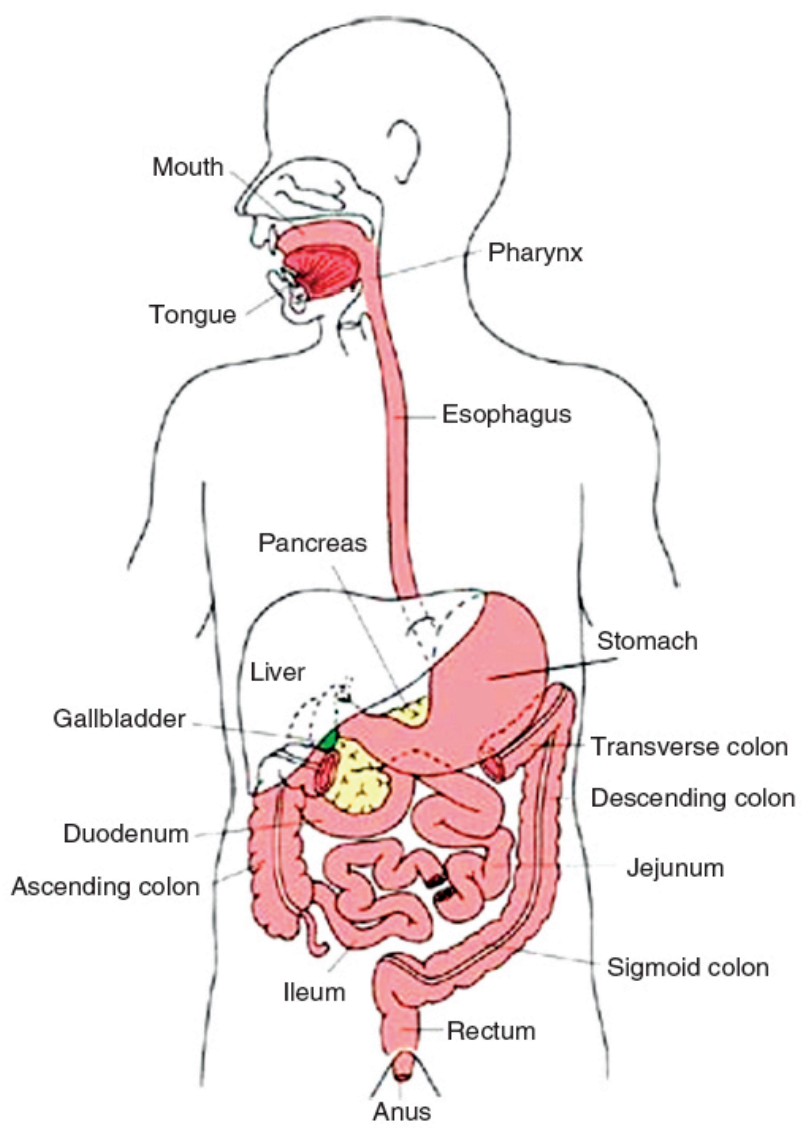

Figure 1.17 General anatomy of gastrointestinal tract. Figure reproduced from (MacFarlane and Stover 2008) with permission of Elsevier Ltd. 


\subsubsection{Oral cavity}

The oral cavity or mouth is the point of entry of food into the digestive systems as well as of a large number of drugs. The primary function of the mouth is mechanical (mastication). The oral mucosa has a protective role during the process of mastication, which exposes the mucosa to compression and shear forces. The oral cavity contains a great variety of microorganisms, whose entry into the body as well as of any potential toxic waste product is limited by the oral epithelium (Washington, Washington et al. 2001).

The mucus and saliva present in the mouth lubricate its surface and constitute a further protection barrier (Marieb and Hoehn 2010). Saliva is composed mainly of water, but also contains mucus, proteins, mineral salts and enzymes (Washington, Washington et al. 2001), including amylase, which initiates the digestive process of polysaccharides such as starch. Following mastication, food mixed with saliva form the food bolus, which is directed to the

oesophagus. In the case of dosage forms that are not meant to dissolve in the mouth, the contact between the dosage form and the oral mucosa is usually brief. 
Table 1.3 Biological and physical parameters of the human gastrointestinal tract (approximate values) (Daugherty and Mrsny 1999; Ashford 2002).

\begin{tabular}{|c|c|c|c|c|c|}
\hline Segment & Surface area & Length & $\begin{array}{c}\text { Residence } \\
\text { time }\end{array}$ & pH & $\begin{array}{c}\text { Major catabolic } \\
\text { activities }\end{array}$ \\
\hline Oral cavity & $100 \mathrm{~cm}^{2}$ & & $\begin{array}{l}\text { Seconds to } \\
\text { minutes }\end{array}$ & 6.5 & Polysaccharidases \\
\hline Oesophagus & $200 \mathrm{~cm}^{2}$ & $23-25 \mathrm{~cm}$ & Seconds & $5-6$ & \\
\hline Stomach & $\begin{array}{c}3.5 \mathrm{~m}^{2} \\
\text { (variable) }\end{array}$ & $\begin{array}{c}0.25 \mathrm{~m} \\
\text { (variable) }\end{array}$ & $5 \mathrm{~min}-2 \mathrm{~h}$ & $\begin{array}{c}1-3.5 \text { (fasted } \\
\text { state) }\end{array}$ & Proteases, lipases \\
\hline Duodenum & $1.9 \mathrm{~m}^{2}$ & $0.20-0.35 \mathrm{~m}$ & $0.5-0.75 \mathrm{~h}$ & $4-5.5$ & $\begin{array}{c}\text { Polysaccharidases; } \\
\text { oligosaccharidases; } \\
\text { proteases; } \\
\text { peptidases, lipases, } \\
\text { nucleases }\end{array}$ \\
\hline Jejunum & $184 \mathrm{~m}^{2}$ & $2.0-2.8 \mathrm{~m}$ & $1.5-2.0 \mathrm{~h}$ & $5.5-7.0$ & $\begin{array}{l}\text { Oligosaccharidases; } \\
\text { peptidases; lipases }\end{array}$ \\
\hline Ileum & $276 \mathrm{~m}^{2}$ & $3.0-4.2 \mathrm{~m}$ & $5-7 \mathrm{~h}$ & $7.0-7.5$ & $\begin{array}{c}\text { Oligosaccharidases; } \\
\text { peptidases; lipases; } \\
\text { nucleases; } \\
\text { nucleotidases }\end{array}$ \\
\hline $\begin{array}{l}\text { Colon and } \\
\text { rectum }\end{array}$ & $1.3 \mathrm{~m}^{2}$ & $1.5 \mathrm{~m}$ & $\begin{array}{c}1-60 \mathrm{~h}(35 \\
\text { hours average })\end{array}$ & $7.0-7.5$ & $\begin{array}{l}\text { Broad spectrum of } \\
\text { bacterial enzymes }\end{array}$ \\
\hline
\end{tabular}

\subsubsection{Oesophagus}

Once food or dosage forms leave the oral cavity, they are transported via the oesophagus to the stomach by a simple or multiple peristaltic waves. The oesophagus is composed of a thick muscular layer whose inner surface is covered with a well-differentiated squamous epithelium of non-proliferative cells. The epithelial cells provide a tough impermeable lining which resists the abrasive nature of food boluses and its mucus protects the lower part of the oesophagus from gastric acid (Ashford 2002). The function of the oesophagus is purely mechanical and no activity related to digestion or absorption occurs at this point of the gastrointestinal tract (Marieb and Hoehn 2010). In the upright position the transit of materials through the oesophagus is assisted by gravity. 


\subsubsection{Stomach}

The stomach is the most dilated part of the gastrointestinal tract and is composed of a thick muscular layer. The main functions of the stomach are to act as a temporary reservoir for ingested food, to mix and reduce ingested solids to a semifluid mass, known as chyme, by the action of acid and enzymatic digestion, and then emptying these contents at a controlled rate into the upper small intestine (duodenum). This enables better contact of the ingested material with the mucous membrane of the intestines and thereby facilitates absorption (Ashford 2002). The stomach can be divided into three anatomical regions: the fundus, the corpus or body, and the antrum. The proximal region, made up of the fundus and corpus, serves as a reservoir for ingested material, which gradually presses the gastric contents forward to the distal stomach and the duodenum, whereas the distal region (antrum) acts as a gastric homogenizer and grinder and it is coordinated with the corpus in the propulsion of gastric contents towards the pylorus (Mayersohn 2002). The opening of the stomach to the to the duodenum is controlled by the pyloric sphincter, which allows liquids and fractions of chyme to empty while other material is retropulsed into the antrum of the stomach and caught up by the next peristaltic wave (contraction of the distal stomach) for further size reduction before emptying (Ashford 2002).

Each section of the stomach is covered with a mucous membrane with a variety of secretory glands located beneath the epithelium and whose distribution varies from one region to another (Washington, Washington et al. 2001). The gastric secretions include (Washington, Washington et al. 2001; Ashford 2002):

- acid $(\mathrm{HCl})$ secreted by the parietal cells, which maintains the $\mathrm{pH}$ of the stomach between 1 and 3.5 in the fasted state;

- gastrin, a hormone that stimulates gastric acid production and aids in gastric motility. The release of gastrin is stimulated by peptides, amino acids and distension of the stomach;

- pepsins, which are proteases whose precursor, pepsinogen, is released by the gastric chief cells (or peptic cells). Pepsins break down proteins into peptides at low $\mathrm{pH}$. The low gastric $\mathrm{pH}$ produced by the $\mathrm{HCl}$ leads to the hydrolysis of pepsinogen into pepsin; Pepsins are more efficient in cleaving peptide bonds between hydrophobic and preferably aromatic amino acids. 
- and mucus, which is secreted by the surface mucosal cells and lines the gastric mucosa. The mucus protects the gastric mucosa from the combination of acid and the proteolytic action of pepsin.

Very little nutrient and drug absorption occurs in the stomach due to its small surface area compared to the small intestine. The rate of gastric emptying can be a controlling factor in the onset of drug absorption from the major absorptive site, which is usually the small intestine (Ashford 2002).

\subsubsection{Small intestine}

The small intestine is the longest and most complex organ of the gastrointestinal tract, extending from the pyloric sphincter of the stomach to the ileocaecal junction, where it joins the large intestine. Its main functions are to mix food with enzymes and other intestinal secretions to facilitate digestion and absorption, and to propel the unabsorbed materials in an aboral direction. Although drugs may be absorbed by passive diffusion or by other absorption mechanisms from all parts of the gastrointestinal tract, for most drugs, the optimum site for drug absorption after oral administration is the small intestine. It is the region of the digestive system that presents the vastest surface area for drugs to diffuse passively (Table 1.3). In addition, the wall of the small intestine has a rich network of both blood and lymphatic vessels. The lymphatic system is important in the absorption of fats from the gastrointestinal tract. Its surface area is increased immensely, by about 600 times that of a simple cylinder, to approximately $200 \mathrm{~m}^{2}$ in an adult, by the ensemble of three distinctive structures, which render the small intestine such a good absorption site: the folds of Kerckring, the villi and the microvilli (Ashford 2002). The folds of Kerckring increase the surface of absorption by 3 times and consist of submucosal coarse folds. These folds are several millimetres in depth and extend circularly most of the way around the intestine, being particularly well developed in the duodenum and jejunum. The villi (Figure 1.18) amplify the surface of absorption by 30 times and are finger-like longitudinal projections into the lumen (approximately $0.5-1.5 \mathrm{~mm}$ in length and $0.1 \mathrm{~mm}$ in diameter). They are covered with an epithelium consisting of a mixture 
of absorptive cells (enterocytes), mucous cells (caliciform cells) and endocrine cells and are well supplied with blood vessels. About 600-1000 of brush-like structures called microvilli ( $\sim 1 \mu \mathrm{m}$ in length and $0.1 \mu \mathrm{m}$ in width) cover each villus, providing the largest increase in surface area. The microvilli are covered with the glycocalyx, a fibrous substance consisting of a mixture of proteins, cholesterol and glycoproteins, and together they form the so-called "brush border", which constitutes an important physical barrier with a powerful enzymatic activity (Washington, Washington et al. 2001; Ashford 2002).

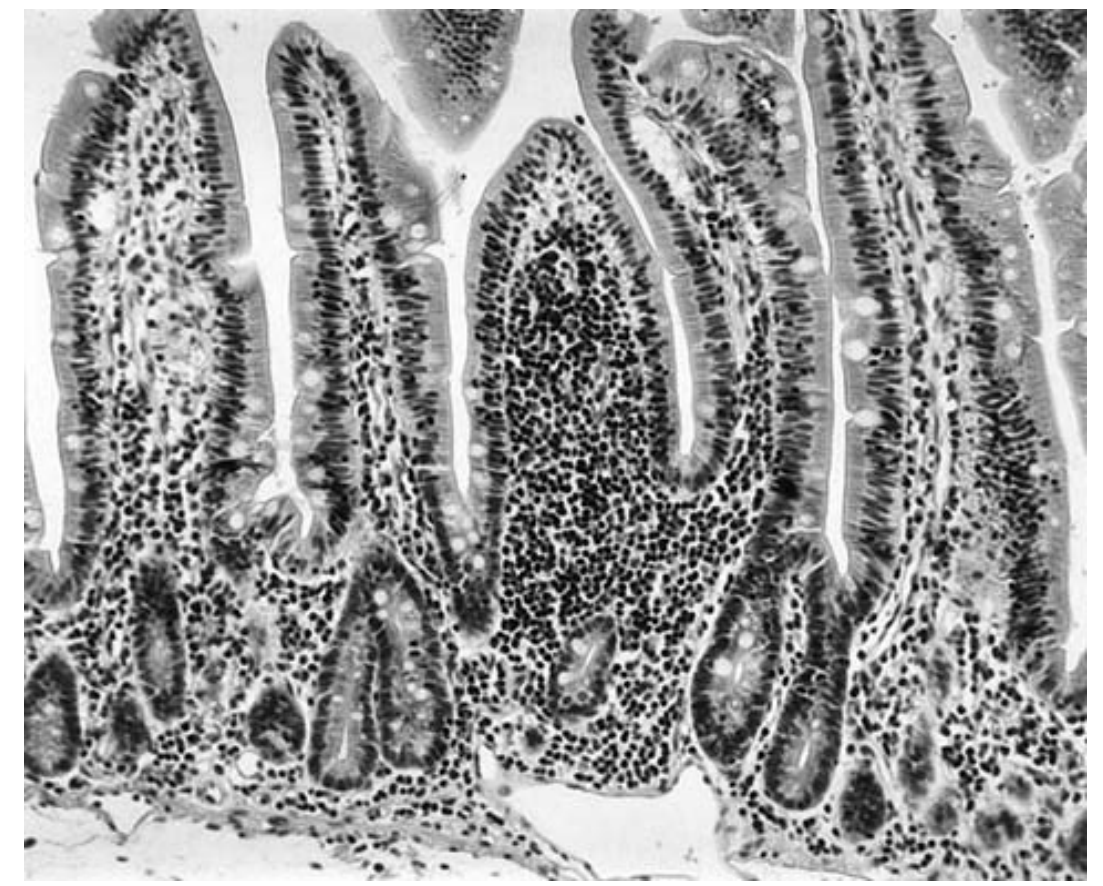

Figure 1.18 Epithelium, lamina propria and villi in the jejunal mucosa. Figure reproduced from (Moghaddami, Cummins et al. 1998) with permission of Elsevier Ltd.

The small intestine is divided into the duodenum, the jejunum, and the ileum (Figure 1.17). These regions are not anatomically distinct, although there are differences in villus height, absorptive capability, specificity and secretion. The duodenum constitutes the first 20$35 \mathrm{~cm}$ and receives the chyme from the stomach. The duodenum wall contains duodenal digestive glands and Brunner's glands. Brunner's glands are only found in the submucosa of 
the duodenum and they produce a protective, bicarbonate rich, alkaline secretion that neutralizes the acidic content from the stomach and provide an alkaline environment for the intestinal enzymes to be active. The jejunum is the main area for the absorption of nutrients and drugs from the gastrointestinal tract into the systemic circulation. It is thicker walled and more vascular than the duodenum and has larger and more numerous villi than the ileum (Washington, Washington et al. 2001; Ashford 2002). In the ileum, the Peyer's patches are larger and more numerous than elsewhere in the intestine. The Peyer's patches are areas of lymphoid tissue close to the epithelial surface, which play a key role in the immune response as they transport macromolecules and are involved in antigen uptake (Ashford 2002).

The ileocaecal junction divides the terminal small intestine from the caecum. Its function seems to be to retain chyme in the small intestine until digestion is largely complete and then to empty its contents into the large intestine. The ileocaecal junction also serves to prevent the spread of the colonic bacteria into the small intestine (Melia, Washington et al. 1994).

The process of digestion depends on the proper functioning of other organs that release their secretions into the small intestine, i.e., the liver and the pancreas. These two organs produce the elements necessary to the degradation and absorption of lipids, carbohydrates and proteins. The role of the liver in the digestion is the production of bile, a complex aqueous mixture of organic solutes (bile acids, phospholipids, cholesterol and bilirubin) and inorganic compounds (plasma electrolytes, sodium and potassium). Bile is stored in the gallbladder and when a meal is ingested, the gallbladder contracts and bile is secreted into the duodenum where it emulsifies dietary fat by the formation of micelles, promoting its efficient absorption (Ashford 2002; Marieb and Hoehn 2010). In the case of the pancreas, this large gland produces and secretes pancreatic juice, which major components are sodium bicarbonate and enzymes. The enzymes consist of proteases, mainly trypsin, chymotrypsin and carboxypeptidases, which are secreted as inactive precursors or zymogens and converted to their active forms in the lumen by the enzyme enterokinase. The pancreatic juice also contains amylase, lipase, esterases, and nucleases. The bicarbonate component is largely regulated by the $\mathrm{pH}$ of chyme delivered into the small intestine from the stomach (Washington, Washington et al. 2001; Ashford 2002). 


\subsubsection{Large intestine}

The large intestine (or large bowel) is the last section of the digestive system and it is about $1.5 \mathrm{~m}$ long. It extends from the ileocaecal junction to the anus and consists of the caecum, colon, rectum, and anal canal. The colon is, in turn, composed of the ascending colon, the hepatic flexure, the transverse colon, the splenic flexure, the descending colon, and the sigmoid colon (Washington, Washington et al. 2001; Ashford 2002). The ascending and descending colons are relatively fixed, since they are attached via the flexures and the caecum. The transverse and sigmoid colons, however, are much more flexible (Ashford 2002).

The main difference between the structure of the mucosa in the large and small intestine is that the first one is devoid of villi. However, the irregularly folded mucosa, the microvilli of the absorptive epithelial cells and the presence of crypts, termed crypts of Lieberkühn, increase the surface area of the colon by 10-15 times that of a simple cylinder of similar dimensions (Washington, Washington et al. 2001; Ashford 2002). Nonetheless, the surface area remains approximately 1/30th that of the small intestine (Ashford 2002). An important number of mucous cells are dispersed within the colonic mucosa, which is thicker and tighter than the mucosa of the small intestine (Marieb and Hoehn 2010).

One of main functions of the colon is the absorption of sodium ions, chloride ions and water from the lumen in exchange for bicarbonate and potassium ions. Thus, the colon has a significant homeostatic role in the body (Caspary 1992; Ashford 2002). The metabolic activity in the colon is carried out by the bacterial microflora and is limited to the fermentation of polysaccharides and proteins which have escaped digestion (Caspary 1992). The large intestine is also responsible for the formation of a solid stool and the storage of faecal matter until it can be discharged. 


\subsubsection{Gastrointestinal transit of pharmaceutical dosage form}

\subsubsection{Introduction}

The transit time of SR dosage forms through certain regions of the gastrointestinal tract may represent a physiological constraint on the use of these pharmaceutical products, given that it limits the residence time at the absorption site and, consequently, the maximum period of time during which a therapeutic response can be maintained following administration of a single dose. If SR drug delivery systems are being designed, it is, thus, important to consider their transit times along each area of the digestive system, amid other factors that will affect their behaviour, such as the gastrointestinal fluids composition and $\mathrm{pH}$ differences. It is also important to take into account that certain situations, such as certain disease conditions or the presence of some drugs can affect gastrointestinal transit.

\subsubsection{Oesophagus}

Normally, transit through the oesophagus is extremely rapid and dependent upon the size, shape and density of the dosage form and the position of the subject. In general, most dosage forms, when taken in an upright position, transit through the oesophagus in about 10 to 15 seconds (Washington, Washington et al. 2001; Ashford 2002). Large oval tablets seem to have a shorter oesophageal transit than large round tablets. However, the size and shape of formulations have little influence on oesophageal transit compared to the posture of the subject. Generally, oesophageal transit is slower in supine patients than upright ones (Washington, Washington et al. 2001). Tablets/capsules taken in the supine position, especially if taken without water, are likely to lodge in the oesophagus. Adhesion to the oesophageal wall can occur as a result of partial dehydration at the site of contact and the formation of a gel between the formulation and the oesophagus. The chances of adhesion will depend on the size, shape and type of formulation. A delay in reaching the stomach may delay the onset of action of the drug or cause damage or irritation to the oesophageal wall, depending that damage on the drug (Ashford 2002). 


\subsubsection{Stomach and gastric retention and emptying}

The time a non-disintegrating dosage form takes to traverse the stomach is usually termed the gastric residence time, gastric emptying time or gastric emptying rate. Gastric emptying of pharmaceuticals is highly unpredictable and depends greatly on the type of dosage form (size and shape) and the presence (fed state) or absence (fasting state) of food in the stomach. Other factors that can influence gastric emptying include the postural position, the composition of the ingested food, the effects of the drugs present in the formulation, such as possible irritancy of the drug on the mucosa, and disease state.

Gastric emptying: interdigestive and digestive phases

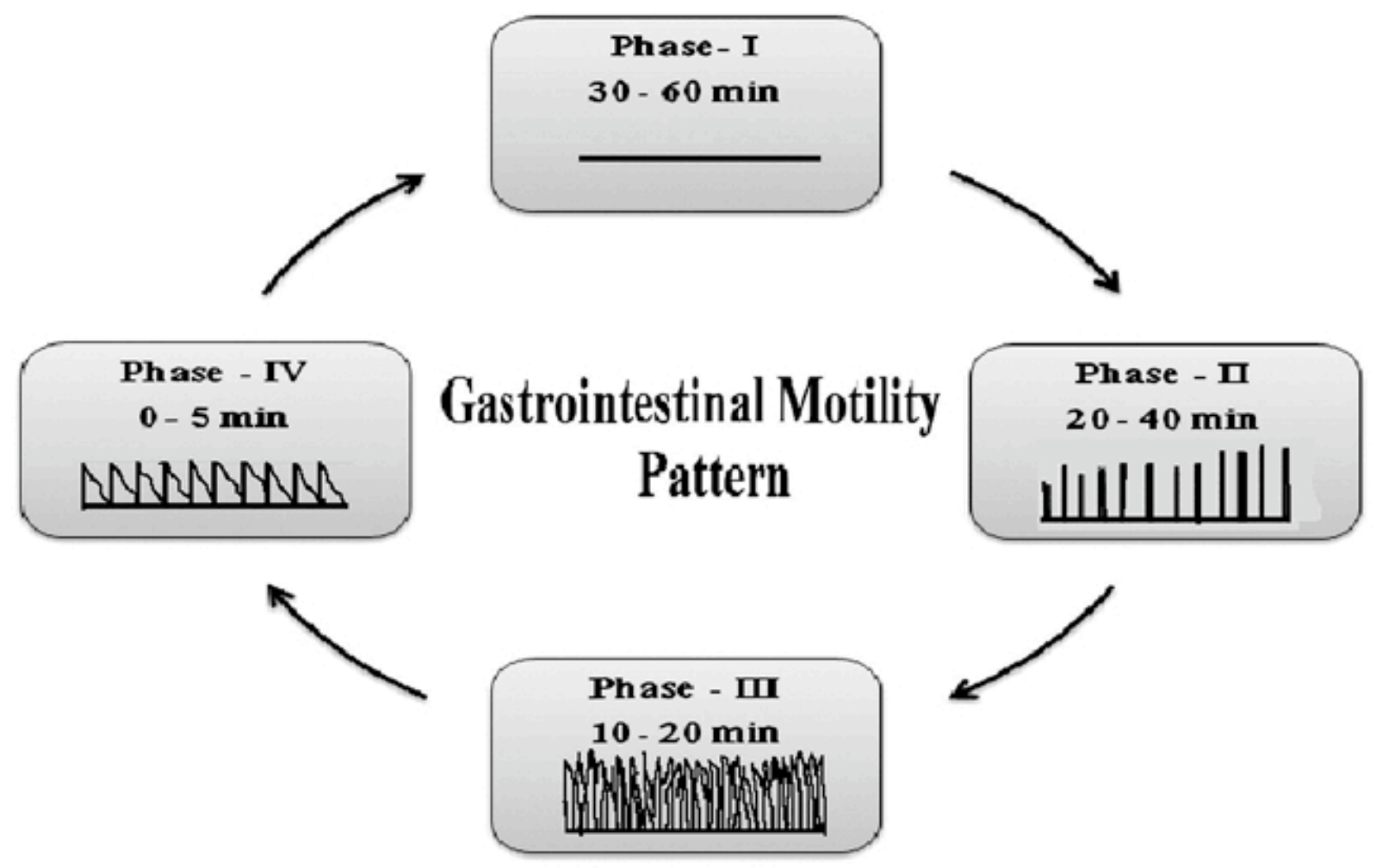

Figure 1.19 Schematic representation of the interdigestive motility pattern, frequency of contraction forces during each phase and average time period for each phase. Figure reproduced from (Prajapati, Jani et al. 2013) with permission of Elsevier Ltd. 
During the interdigestive phase, the fasted stomach exhibits a cyclic activity called the migrating motor complex (MMC) (Figure 1.19), which governs the transit of dosage forms. Each cycle can be divided into four phases. Phase I, the most quiescent, develops few or no contractions for 30 to 60 minutes. Phase II, which lasts 20 to 40 minutes, is characterized by the gradual increase of the incidence of irregular and intermittent sweeping contractions simultaneously in the antrum and duodenum, culminating in the onset of phase III. During phase III intense bursts of circular peristaltic waves start in the stomach and sweep the residual material and bacteria rapidly down the entire small intestine into the colon. These powerful peristaltic contractions, which last about 10 to 20 minutes and are often called the housekeeper waves, open the pylorus and clear the stomach of any residual material, including existing large non-disintegrated tablets and capsules. These waves then subside to phase IV, a short transitional period of decreasing activity until next cycle (Kelly 1980; Sarna 1985; Ashford 2002; Prajapati, Jani et al. 2013). The different phases of the cycle migrate distally from the stomach to the duodenum and further proceed from the small intestine through the colon (Sarna 1985; Kellow, Borody et al. 1986). The whole cycle repeats itself every 1.5 to 2 hours until a meal is ingested, at which stage the MMC cycle is immediately interrupted (Code and Marlett 1975; Thompson, Wingate et al. 1980) and the digestive phase initiated (Malmud, Fisher et al. 1982). Therefore, the emptying of individual units from the fasted stomach is extremely erratic, occurring any time between 10 minutes and 3 hours after administration, depending on the occurrence of the next MMC relative to the time of dosing (Melia, Washington et al. 1994).

When food is ingested, the proximal stomach relaxes to receive it and gradual contractions of this region move the contents distally. The distal part of the stomach develops peristaltic contractions moving as a ring from the mid-stomach toward the antrum to the pylorus, which mix and break down food particles and move them towards the pyloric sphincter. The pyloric sphincter allows liquids and small food particles to empty, while the solids unable to pass forward are retropulsed back into the antrum of the stomach and caught up by the next peristaltic wave for further size reduction before emptying (Moes 1993; Ashford 2002). Food intake also induces changes in the gastrointestinal environment, including lumen content, $\mathrm{pH}$, viscosity, ionic strength, osmolality, secretions (bile salts, digestive enzymes, etc.), metabolic enzymes, and transporters (Qiu 2009). 
Close parallels are found between the gastric transit of the different food components and their pharmaceutical formulation counterparts. In the fed state, liquids, pellets and disintegrating tablets will tend to empty with food, whereas large SR dosage forms can be retained in the stomach for long periods of time (Melia, Washington et al. 1994; Ashford 2002). However, there is no certitude regarding the exact size of a dosage form that would not be expelled until the MMC arrival. It has been reported that non-disintegrating dosage forms up to $7 \mathrm{~mm}$ in diameter can still be emptied from the human stomach during the postprandial period (Park, Chernish et al. 1984; Khosla, Feely et al. 1989). A non-disintegration dosage form greater than $10 \mathrm{~mm}$, on the other hand, will have to wait until the end of digestion before it can be cleared from the stomach into the small intestine in association with the phase III cleansing contractions of the MMC activity (Smith and Feldman 1986). The cut-off size of solids that are retained in a fed stomach is also influenced by the inter-individual variations in the diametrical opening of the pylorus and in the pressure force of the propelling waves. In addition, gastric emptying is proportional to meal size and composition. Emptying of a large single-unit dosage form after a light meal has been reported to happen at 2 to 3 hours after administration (Melia, Washington et al. 1994), whilst if the same dosage form is given with a large meal and the subject is fed at regular intervals, the dosage form can remain in the stomach for over 16 hours (Mojaverian, Ferguson et al. 1985) owing to prolonged suppression of the MMC. The longer gastric residence time will delay drug absorption.

The gastric residence time of non-disintegrating monolithic dosage forms in the fasted state is, thus, mainly dependent on the coincidence between dosing time and phase III occurrence. The gastric emptying is, however, rather unpredictable and the residence time is usually short and highly variable (Moes 1993). Consequently, the fasting mode of administration of large SR dosage forms has the disadvantage of offering only a limited period of time for effective drug-release in the upper tract. As a result, reduced drug bioavailability is likely to occur, particularly if the optimum absorption region is in the small intestine (Welling and Dobrinska 1987). 


\subsubsection{Small intestine}

The small intestine is the main site of absorption in the gastrointestinal tract for most drugs. Therefore, small intestinal transit time is an important parameter as the extent of drug absorption from the small intestine is directly related to the length of time that the drug remains in contact with the absorptive epithelium.

The average small intestine transit duration is approximately three hours (Davis, Hardy et al. 1986; Davis, Hardy et al. 1986; Khosla, Feely et al. 1989; Ashford 2002). Studies on whether the presence of food influences intestinal transit times have been contradictory, with some authors reporting variations depending on whether the subject has eaten and the size of the meal (Read and Sugden 1988; Melia, Washington et al. 1994) and others stating that, in contrast to the stomach, the small intestine does not discriminate between solids and liquids, and hence between dosage forms (e.g. solutions, pellets and single units) or between the fed and the fasted state (Davis, Hardy et al. 1986; Khosla, Feely et al. 1989; Moes 1993; Ashford 2002).

The discrimination between the fed and the fasted state and dosage forms can be explained based on the fed and fasted patterns of motility in the small intestine. The fasting motility pattern is a continuation of the MMC initiated in the stomach. For most of the fasting period the majority of the small intestine is at rest. At approximately 90-120 minute intervals, an intense contraction sweeps from the duodenum to the ileum, taking approximately 90 minutes to complete its journey. As one contraction ends, another begins. These contractions serve to sweep the stomach and small intestine of material that has no nutritional value, quickly moving it to the colon. The fed pattern of motility is random and consists of segmental and peristaltic contractions, with the segmental contractions being the most frequent. The segmental motility is characterized by reversible contractions of adjacent segments of the bowel, less than $2 \mathrm{~cm}$ in length. This type of motility mixes chyme by continually moving it in the lumen and increasing the contact with the absorbing surface. Since there are less frequent segmental contractions aborally than orally, there is a net movement of chyme towards the large intestine. This movement is enhanced by peristaltic contractions that occur less frequently than segmental contractions and each of them move the chyme a few centimetres (Melia, Washington et al. 1994). Therefore, there are two main types of intestinal movement, 
propulsive and mixing. The propulsive movements primarily determine the intestinal transit rate and, consequently, the residence time of the drug or dosage form in the small intestine (Melia, Washington et al. 1994; Ashford 2002).

The physical and chemical nature of the food determines the number of small intestinal contractions and, thus, the transit through the small intestine. For example, large amounts of unabsorbable carbohydrate or a large amount of fluid accelerate transit and could, therefore, reduce the degree of absorption from a SR formulation taken with the meal (Read, Miles et al. 1980). The presence of fat in the distal small intestine, on the other hand, prolongs transit time and may, therefore, increase the degree of absorption from SR dosage forms by increasing their residence time in the upper gastrointestinal tract. However, unlike the stomach, the intestine does not appear to discriminate between liquids and small solid particles (Melia, Washington et al. 1994). Small intestinal residence time is particularly important for SR dosage forms, because when administered after a light breakfast, for example, these dosage forms can reach the large intestine after 4-6 hours. The motor activity of the upper small intestine also differs from that in the ileum. The luminal contents travel rapidly through the jejunum to the ileum, where they may remain until transported gradually into the colon (Read, Al-Janabi et al. 1986).

Non-disintegrating matrices may remain at the ileocaecal junction for some time. Times ranging from 2-20 hours in the ileocaecal region have been reported (Marvola, Aito et al. 1987).

\subsubsection{Large intestine}

The transit time of dosage forms through the large intestine, in particular through the colon is long and variable, ranging from anything between 2 and 48 hours, and depends on the type of dosage form, diet, eating pattern and disease state (Ashford 2002). The colonic transit is mainly aboral and is characterized by short bursts of activity followed by long periods of stasis. The contractile activity in the colon can be divided into propulsive contractions or mass movements, which are associated with the aboral movement of contents, and segmental contractions, which serve to mix the luminal contents and result in only small aboral 
movements. Segmental contractions are caused by contraction of the circular muscle and prevail, whereas the propulsive contractions are due to contractions of the longitudinal muscle and occur only 3-4 times daily in normal individuals (Ashford 2002).

As a result of the usually long transit time through the colon, a SR dosage form is likely to spend a long time, if not most of its time, within the large intestine. Therefore, transit through the colon is an important parameter in optimizing delivery of drugs by SR dosage forms for prolonging drug absorption.

It is expected that absorption of most drugs from the colon, by comparison with that in the small intestine, will be slower, because the colonic mucosa has a much lower and more flat surface area, with fewer folds and without villi. In addition, the lumen of the colon is wider, the movement more sluggish and the volume of dissolution fluid available is low. Apart from transit constraints, drugs also encounter transverse resistance to absorption in the colon (Melia, Washington et al. 1994). However, many drugs appear to be well absorbed from the colon as the low surface area is balanced by the prolonged residence time in this region. In fact, studies have demonstrated that the model drugs used in the studies of the present thesis, acetaminophen (Ishibashi, Ikegami et al. 1999) and tramadol hydrochloride (Lintz, Barth et al.

1998) are quickly and well absorbed from the colon. This is important because it enhances the likelihood of a complete absorption of these drugs from the developed dosage form.

\subsubsection{Barriers to drug absorption}

The mechanisms necessary for the digestion of food and the absorption of nutrients, and for the protection of the intestinal membrane represent potential barriers to the passage of exogenous molecules into the systemic circulation. Next, a description of some physiologic parameters that may influence intestinal drug absorption is given. 


\subsubsection{The environment within the lumen}

In general, a drug substance must be in solution form before it can be absorbed through the absorptive epithelia of the gastrointestinal tract into the body fluids. Besides, it cannot become bound to food or other material within the gastrointestinal tract, and it needs to be chemically stable in the $\mathrm{pH}$ gradient and resistant to enzymatic degradation along the gastrointestinal tract (Ashford 2002). The gastrointestinal pH may influence the chemical stability of the drug in the lumen, its dissolution or its absorption, if the drug is a weak electrolyte. The digestive enzymes found in the gastrointestinal tract may degrade drugs or dosage forms that resemble nutrients (Ashford 2002), as it is the case of the degradation of peptide drugs by pepsin and proteases, or of starch-based dosage forms by amylases. In addition, the intestinal microflora can metabolize a wide variety of pharmacological agents resulting in production of metabolites required for the physiological activity of these agents or conversely in the inactivation of these agents (Goldin 1990). The presence of food in the gastrointestinal tract can also influence the rate and extend of absorption via a variety of mechanisms, some of which, such as the modification of the gastric $\mathrm{pH}$ and emptying time and the stimulation of gastrointestinal secretions, were described in previous sections. Other mechanisms include competition between food nutrients and drugs with a similar chemical structure for specialized absorption mechanisms, or the irreversible complexation of drugs with food components as, for example, tetracycline and calcium (Ashford 2002).

\subsubsection{Mucus}

The gastrointestinal epithelium is covered by a continuous layer of protective mucus gel that a drug must first diffuse through before it can be absorbed across the epithelium (Thomson and Dietschy 1977). In performing its functions, mucus may adversely affect the absorption of drugs administered by the oral route. The diffusion coefficient of a drug through

mucus depends on the relative size of the drug molecule, the effective mesh size of the mucous gel formed by the association of the mucin macromolecules, and any interactions between the drug and the mucous components. For example, an inverse relationship between 
molecular weight and diffusion coefficient of the drug has been observed. In addition, the permeability of small, uncharged molecules appears to not be as significantly affected by a mucous barrier as larger or charged (cationic) molecules, which can form ionic bonds with negatively charged mucins (Khanvilkar, Donovan et al. 2001).

\subsubsection{Gastrointestinal membrane and drug transport mechanism}

The gastrointestinal membrane separates the lumen of the stomach and the intestines from the systemic circulation, and is the main cellular barrier to the drug absorption. As for all other cell membranes, the plasma membrane in the intestinal epithelial cells consists of lipids, proteins, lipoproteins and polysaccharides. The elemental structure of the membrane, usually known as the fluid mosaic model (Figure 1.20), is the phospholipid bilayer, which forms a stable barrier between two aqueous compartments, i.e., the inside and the outside of the cell. The barrier has the characteristics of a semipermeable membrane, allowing the rapid transit of some materials and restraining the passage of others. Proteins embedded within the phospholipid bilayer carry out the specific functions of the plasma membrane, including selective transport of molecules (Cooper 2000; Ashford 2002).

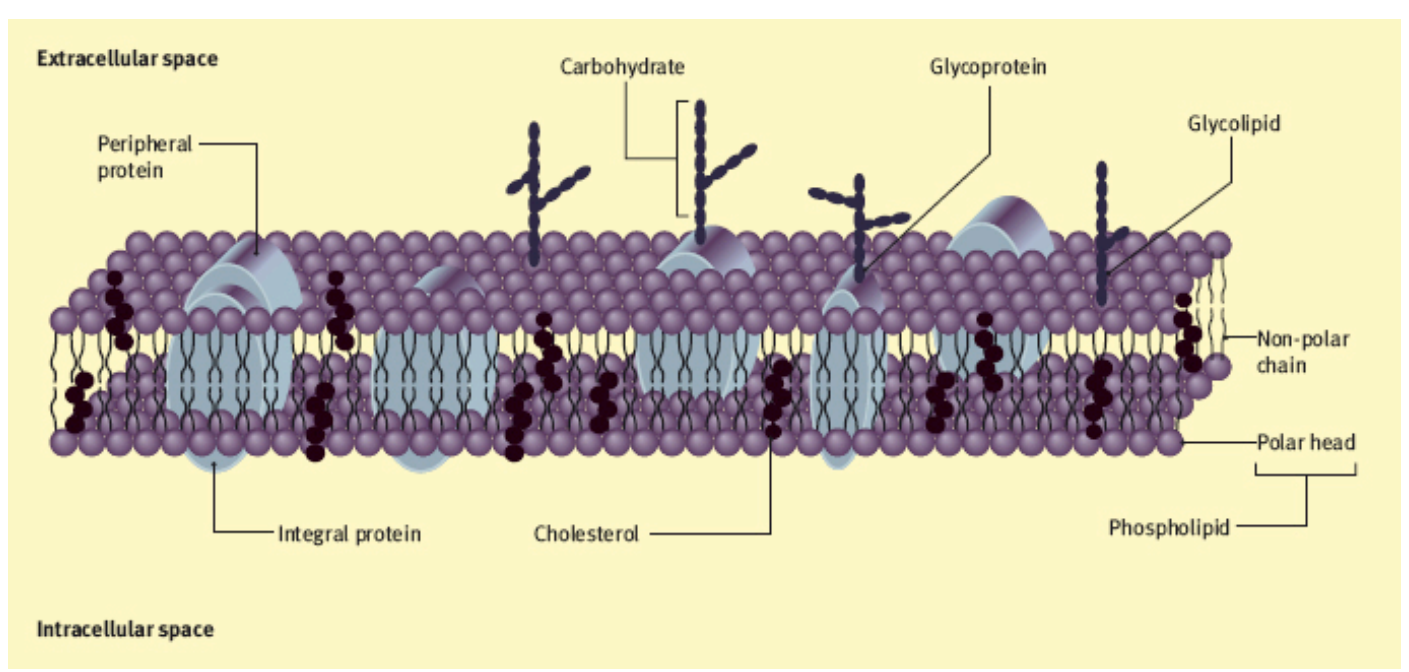

Figure 1.20 Fluid mosaic model of cell membranes (epithelial and other cell membranes) with the integral and peripheral proteins embedded in the phospholipid bilayer. Figure reproduced from (Fry 2007) with permission of Elsevier Ltd. 
Absorption across the gastrointestinal epithelium is mediated by transcellular (across the cells) and paracellular (through the intercellular spaces) mechanisms. The transcellular pathway (Figure 1.21) involves passive or active movement across cell membranes and is divided into passive transport (simple diffusion and diffusion via pores in the plasma), carriermediated transport (facilitated diffusion and active transport), and endocytosis (Ashford 2002). Paracellular transport (Figure 1.22) involves the movement of drugs between cells also by passive diffusion (Thummel, Kunze et al. 1997). Endocytosis is a process by which cells absorb molecules by incorporating them into the interior of vesicles resulting from the invagination of the cellular membrane. After detachment from the membrane, the vesicles fuse with the lysosomes, which, more often than not, lead to the degradation of the drug (Hillery, Lloyd et al. 2001).

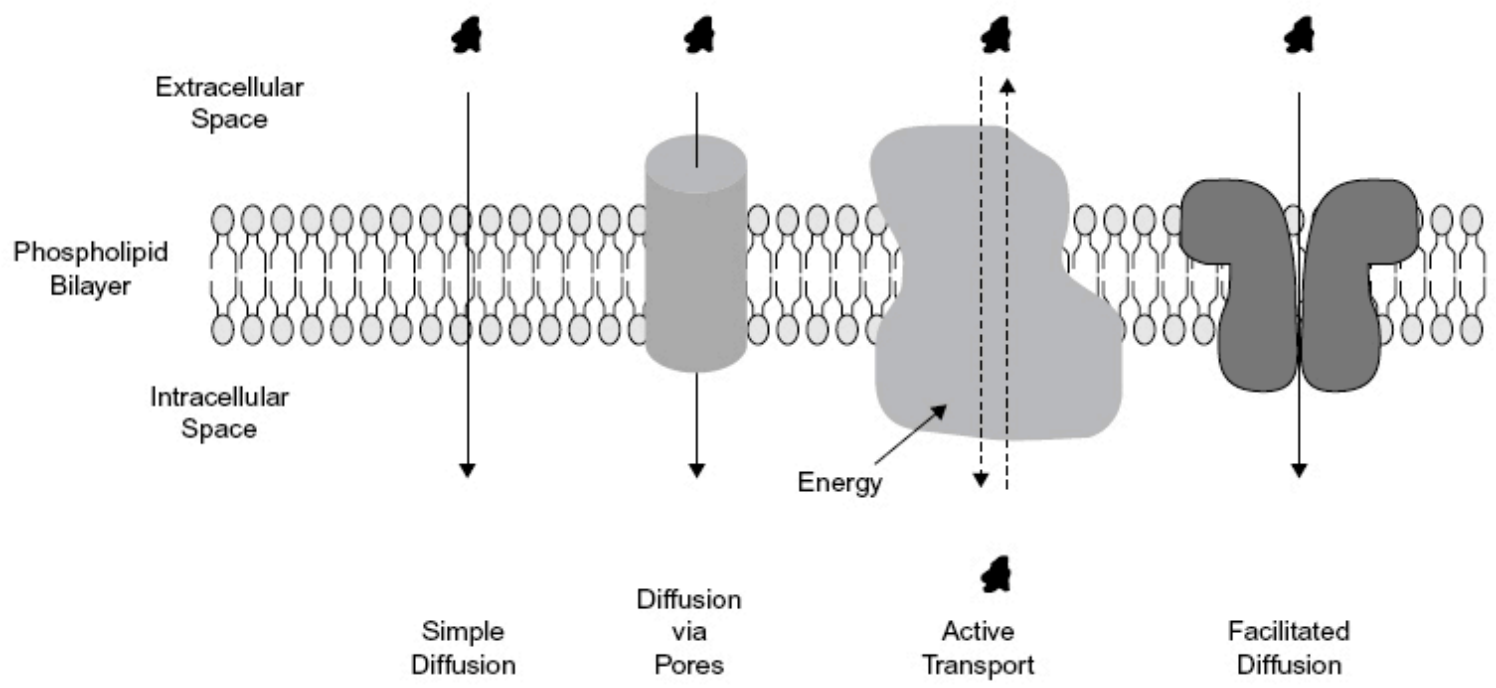

Figure 1.21 Passive transport (simple diffusion or diffusion via pores) and carrier-mediated transport (facilitated diffusion and active transport) across the plasma membrane. Figure reproduced from (Baynes and Riviere 2010) with permission of Elsevier Ltd. 


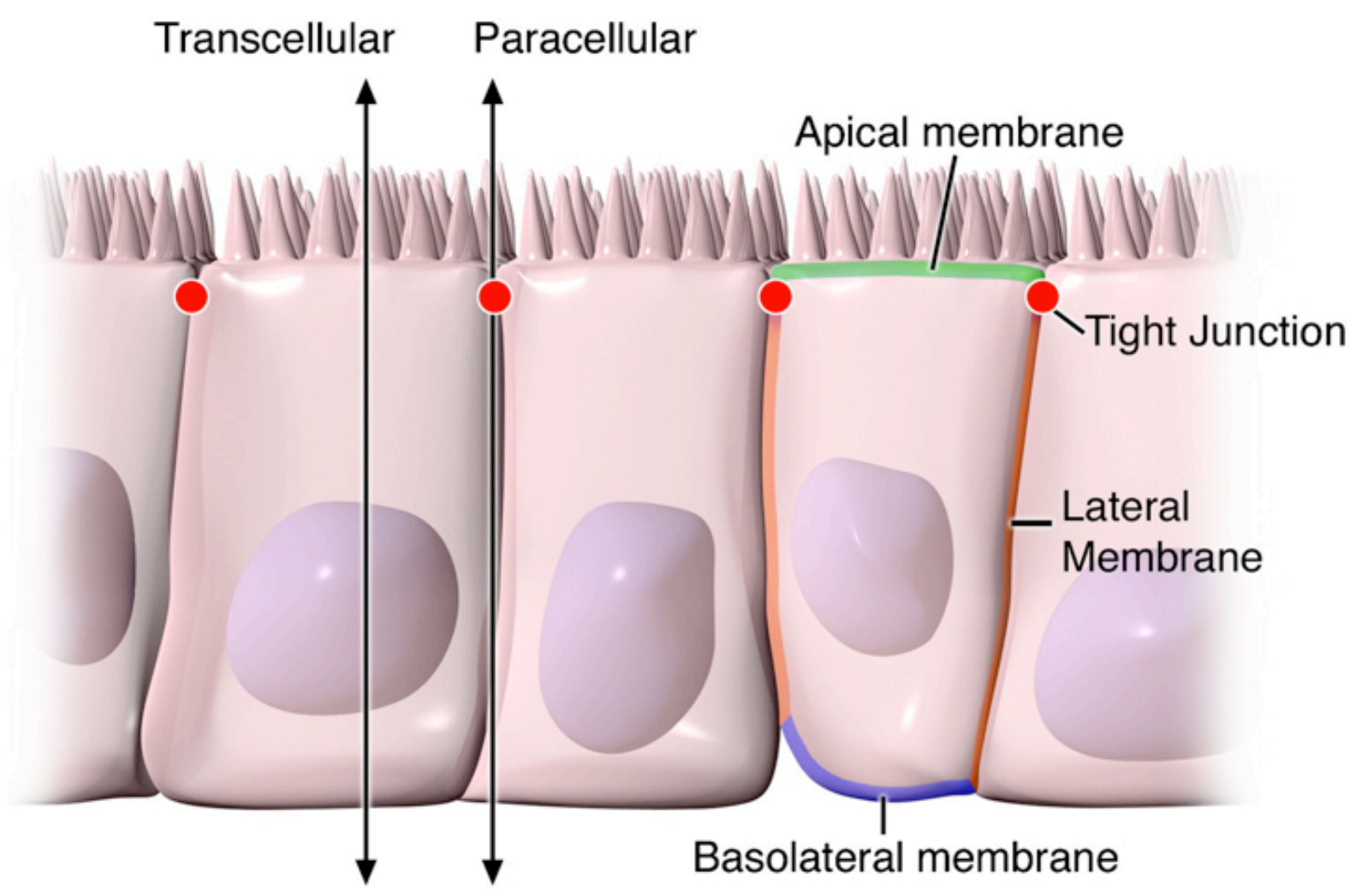

Figure 1.22 Pathways of epithelial permeability. Transcellular permeability is associated with solute or water movement through intestinal epithelial cells. Paracellular permeability is associated with movement in the intercellular space between epithelial cells and is regulated by tight junctions (one of the components responsible for the contact between epithelial cells and for the regulation of transport between extracellular and intracellular space). Figure reproduced from (Groschwitz and Hogan 2009) with permission of Elsevier Ltd.

Passive diffusion is the free diffusion of relatively small lipophilic molecules, and thus many drugs, across the lipoidal membrane, from a region of high concentration in the lumen (apical side) to a region of lower concentration in the blood (basolateral side). Blood flow and a rapid distribution of the drug maintain a lower concentration at the basolateral side than at the absorption site. The rate of transport is determined by the physicochemical properties of the drug, the nature of the membrane and the concentration gradient of drug across the membrane (Ashford 2002). The rate of diffusion can be determined mathematically by Fick's law of diffusion (Hillery, Lloyd et al. 2001): 


$$
\frac{d Q}{d t}=\frac{D k \cdot A \cdot \Delta C}{h}
$$

Equation 10

where $d Q / d t$ represents the rate of drug diffusion across a membrane; $D$, the diffusion coefficient and $k$, the partition coefficient of the drug in the membrane; $h$, the membrane thickness; $A$, the surface area available for absorption; and $\Delta C$, the concentration gradient between the two sides of the membrane.

Facilitated diffusion is as well a passive mechanism. However, it implies the link between the drug and a membrane transport protein, and thus it is reserved to the molecules that present a strong structural homology with the receptor substrate (Hillery, Lloyd et al. 2001). The movement occurs down a concentration gradient and, just as passive diffusion, it does not require an input of chemical energy. In contrast, active transport moves subtracts against a concentration gradient and, therefore, uses cellular energy, such as from adenosine triphosphate (ATP) hydrolysis.

Drug transporters can be generally separated into two major classes - uptake and efflux transporters. Uptake transporters act by facilitating the transfer of drugs into cells. By contrast, efflux transporters transport drugs from the intracellular to the extracellular milieu, often against high concentration gradients (Ho and Kim 2005).

Targeted localization of transporter proteins is involved in the intestinal absorption process as well as in the urinary or biliary excretion of many drugs. These drug transporters tend to be multifunctional and often have normal physiologic roles in terms of transporting endogenous substances such as sugars, lipids, and hormones. They are an important class of proteins for regulating cellular and physiologic solute and fluid balance (Ho and Kim 2005). Both carrier-mediated transport processes are saturable, and can be inhibited and induced. Induction as well as inhibition of carriers involved in drug transport may lead to diminished or enhanced absorption of drugs with affinity for these carriers (Wagner, Spahn-Langguth et al. 2001). 
In the small intestine, enterocytes possess a number of transporters essential for absorption of dietary constituents and drugs. However, among these proteins there is an efflux transporter which can actively pump drugs back into the intestinal lumen and which has been determined to be particularly important in effectively limiting the extent of drug absorption (bioavailability). This multidrug resistance 1 gene $(M D R l)$ product, known as P-glycoprotein (Pg-p), is a transmembrane efflux protein which was first described in tumour cell lines displaying cross-resistance to various anticancer agents (Juliano and Ling 1976). It acts as an ATP-dependent pump that expels drugs out of cells (Chin, Pastan et al. 1992). In addition to tumour cells, Pg-p is present in normal tissues (Thiebaut, Tsuruo et al. 1987; Cordon-Cardo, O'Brien et al. 1990), such as on the apical (luminal) side of the epithelial cells in the intestine, liver, kidney and pancreas (Thiebaut, Tsuruo et al. 1987). Therefore, it plays a significant role in regulating absorption, excretion, and tissue distribution of many drugs, as well as in detoxification processes (Wacher, Salphati et al. 2001).

Substrates for Pg-p cover a broad range of structures with diverse therapeutic indications (Benet, Izumi et al. 1999). For example, studies have indicated that acetaminophen, one of the model drugs used in the present study, is a Pg-p substrate and, therefore, Pg-p is involved in acetaminophen transport (Manov, Bashenko et al. 2006). However, reports on the functional interaction of Pg-p and tramadol, the second model drug used in the study, have been contradictory, with some authors suggesting that tramadol is not a Pg-p subtract (Kanaan, Daali et al. 2009; Hamidi, Sheikholeslami et al. 2012) and others pointing out that tramadol is one of the Pg-p substrates (Slanar, Nobilis et al.). However, both drugs are mainly absorbed by passive diffusion and, therefore, the physicochemical aspects related to drug bioavailability are the most important to take into account.

\subsubsection{First-pass metabolism}

In addition to the physical barrier consisting of mucus and the cellular membrane, a myriad of enzymes also represent a barrier to drug absorption. Orally administered drugs and other xenobiotics can be metabolized by different enzymes in the enterocytes or during their passage through the liver. In general, drug-metabolizing enzymes protect the body against the 
harmful exposure to xenobiotics as well as certain endobiotics (Niwa, Murayama et al. 2009). Together, these metabolic processes that the drug undergoes before reaching the systemic circulation constitute the first-pass metabolism, also known as first-pass effect (Thummel, Kunze et al. 1997).

First-pass metabolism of drugs and other exogenous compounds involves sequential reactions occurring in two phases. Phase I reactions are broadly grouped into three categories, oxidation, reduction, and hydrolysis, though oxidation by the cytochrome P450 (CYP) superfamily of microsomal enzymes is the most common phase I reaction. These reactions aim to increase the polarity of the compound. They are followed by phase II reactions in which the metabolite resulting from phase I is conjugated to a polar ligand. In general, conjugation with phase II enzymes further increases hydrophilicity, and thereby enhances excretion in the bile and/or the urine and, as a result, a detoxification effect (Schuetz, Beck et al. 1996; Xu, Li et al. 2005).

The CYP superfamily of enzymes can be classed in families (e.g., CYP2) and subfamilies (e.g., CYP2D), which include numerous isoenzymes or isotypes (e.g., CYP2B6, CYP3A4) (Thummel, Kunze et al. 1997; Xu, Li et al. 2005). In the digestive system, CYP enzymes are found mostly in the hepatocytes (Wacher, Wu et al. 1995; Wacher, Salphati et al. 2001). However, it was demonstrated that enzymes of the CYP3A subfamily - considered the major phase I drug-metabolizing enzymes in humans (Wacher, Wu et al. 1995) - are expressed at high levels in the mature villus enterocytes of the small intestine (Kolars, Lown et al. 1994; McKinnon, Burgess et al. 1995), and thus have an essential role in the absorption process. CYP3A comprises 30\% (Shimada, Yamazaki et al. 1994) and 70\% (Watkins, Wrighton et al. 1987; Kolars, Lown et al. 1994) of total CYPs in the human liver and intestine, respectively. Among the CYP3A subfamily, CYP3A4 is the most abundant and most important enzyme in human drug metabolism (Benet, Izumi et al. 1999; Wacher, Salphati et al. 2001; Zhang and Benet 2001). Indeed, immunohistochemical studies suggest that the major CYP3A protein present in the liver, jejunum, colon and pancreas is CYP3A4 (Zhang and Benet 2001).

CYP3A4 substrates include a large number of structurally diverse drugs, covering a wide therapeutic range (Smith and Jones 1992). Two examples of CYP3A4 substrates are the model drugs used in this study, acetaminophen and tramadol. 


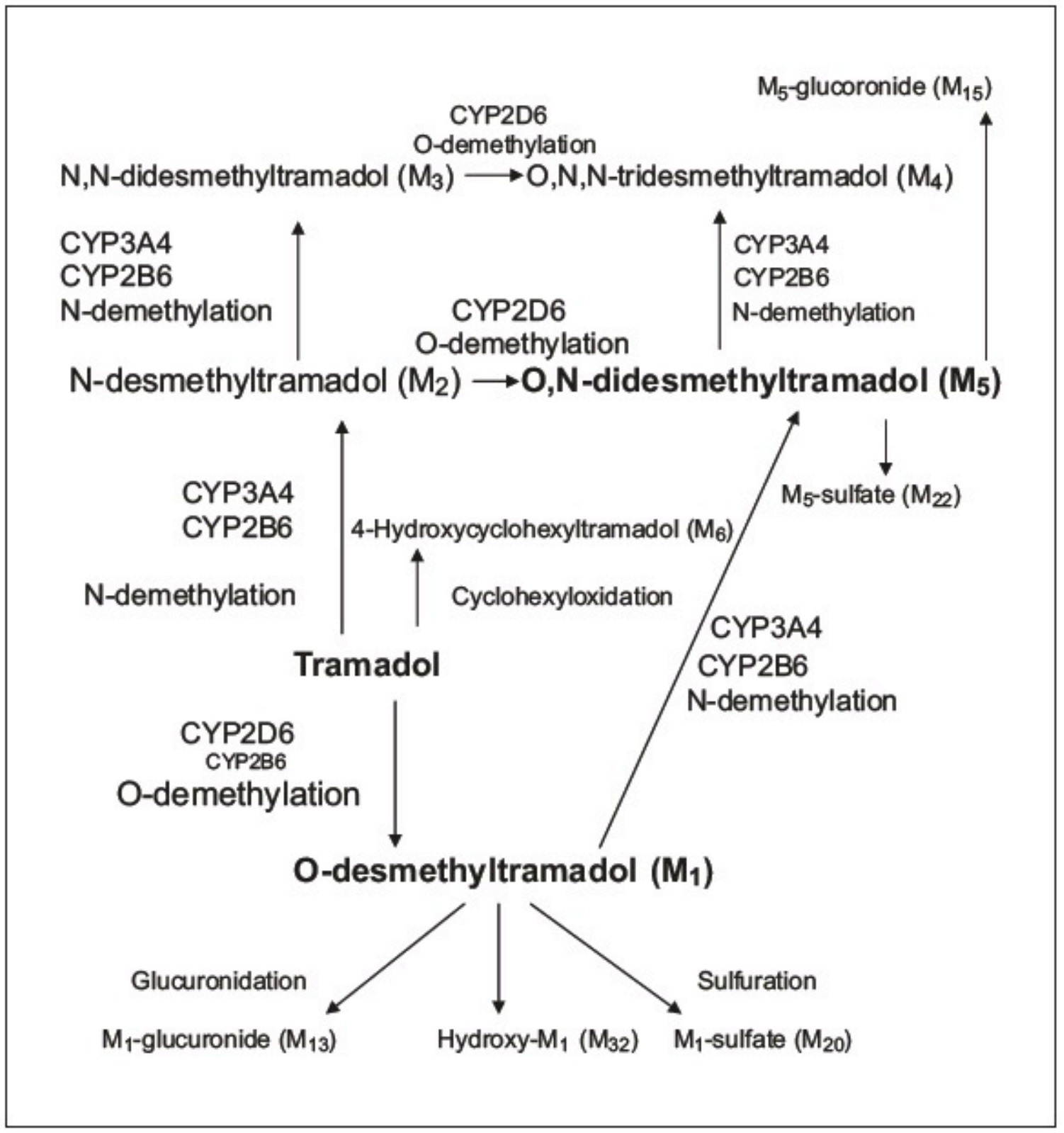

Figure 1.23 Main metabolic pathways of tramadol. The main compounds responsible for tramadol analgesia are marked in bold. Figure reproduced from (Leppert 2011) with permission of Karger Publishers. 
Tramadol undergoes biotransformation in the liver, first by the phase I reactions (mainly O- and N-demethylation), followed by the phase II reactions (mainly conjugation of O- and N-demethylated compounds) to form glucuronides and sulfates (Figure 1.23) (Lintz, Erlacin et al. 1981). The O-demethylation of tramadol leads to the formation of its main metabolite, O-desmethyltramadol (M1), which shows potent analgesic activity, and the Ndemethylation to N-desmethyltramadol (M2). Multiple CYP enzymes are involved in the metabolism of tramadol to both M1 and M2. CYP2D6 appears to be primarily responsible for M1 formation, whereas M2 formation is catalyzed by CYP2B6 and CYP3A4 (Subrahmanyam, Renwick et al. 2001). M1 and M2 may then be further metabolized to secondary metabolites, including O,N-didesmethyltramadol (M5), which exhibits analgesic activity but weaker than M1. All other metabolites are pharmacologically inactive (Leppert 2011).

Regarding the metabolism of acetaminophen, the principle routes of elimination are glucuronidation and sulfation in the liver although oxidation also occurs (Bessems and Vermeulen 2001). Glucuronidation accounts for about $45-55 \%$ of acetaminophen dose (Bessems and Vermeulen 2001; Kiang, Ensom et al. 2005). Glucuronidation is an important conjugation reaction that increases the water solubility of endogenous and exogenous compounds (drugs and their metabolites) and enhances their excretion via urine or bile. This step can be catalyzed by a series of UDP-glucuronosyltransferases (UGTs), which are responsible for the process of glucuronidation, a major part of phase II metabolism. Examples of UGTs implied in the metabolism of acetaminophen are UGT1A1 and UGT1A6 in the liver (Court, Duan et al. 2001; Kiang, Ensom et al. 2005) and UGT1A10 in the intestine (Kiang, Ensom et al. 2005). About 30-35\% of metabolism of acetaminophen occurs via sulfation, another phase II enzyme reaction. In adult human liver, sulfation of acetaminophen is catalyzed by sulfotransferases (SULT), such as SULT1A1 and SULT1A3 (Kiang, Ensom et al. 2005). 


\subsection{Considerations in the selection of drug candidates for incorporation in sustained drug-release dosage forms}

Both the drug-release pattern from a dosage form and the performance of the dosage form in the body are a function of the physicochemical and the biological properties of the drug. Therefore, a number of drug characteristics have to be considered when evaluating drug candidates for oral SR dosage forms. Although, for the purpose of this discussion, the properties of a drug will be described as being either physicochemical or biological, it is important to keep in mind that the biological properties of a drug are a function of its physicochemical properties. For example, the physicochemical properties of drug substances influence greatly their passage into solution and their transport across biological membranes, and thus have a major impact on their bioavailability. Therefore, it is often necessary to consider the various properties as combined factors in order to determine the viability of a candidate drug for a SR product.

\subsubsection{Physicochemical properties of drugs influencing their viability as candidates for sustained drug-release dosage forms}

\subsubsection{Aqueous solubility and dissolution rate}

As a drug must be in solution to be absorbed, drugs that possess very low aqueous solubility usually experience oral bioavailability problems, even from IR systems. They may suffer inconsistent drug absorption caused by difficulty in achieving adequate solubility at or above their main absorption sites along the gastrointestinal tract. A second issue that these drugs create in the formulation of oral SR dosage forms where the release mechanism is diffusion is that, in this case, the concentration in aqueous solution is the driving force for gradual dissolution and release of the drug from the dosage form along the gastrointestinal tract (Langer and Wise 1984; Li, Robinson et al. 1987). The choice of release mechanism for a SR dosage form is, therefore, conditioned by the aqueous solubility of the drug. In addition, drugs with limited aqueous solubility, which have their absorption limited by their dissolution 
rate, do not usually require any additional control over their dissolution rate. These drugs may be good candidates for $\mathrm{CR}$ systems designed to make their dissolution rate more uniform rather than reducing it (Langer and Wise 1984; Gupta and Robinson 1992).

The most widespread and effective strategy used to increase solubility and dissolution rates of acidic and basic drugs is salt formation (Morris, Fakes et al. 1994; Serajuddin 2007). Another technique is the design of bioreversible, more water-soluble derivatives of drugs with low solubility, generally called prodrugs (Stella and Nti-Addae 2007; Jana, Mandlekar et al. 2010). For some drugs, solubility and dissolution may be increased in the fed state because of the presence of micellar solubilizers (Luner, Babu et al. 1994).

Effective SR of drugs with very high aqueous solubility, such as greater than $1 \mathrm{~g} / 10$ $\mathrm{mL}$, may also be very difficult to achieve. Efficient control of the release of such drugs over an extended period of time will depend on the dosage form approach chosen. For example, when such drugs are incorporated in matrix systems, a high proportion of polymeric matrix to drug may be required to produce the SR effect (Langer and Wise 1984). Accordingly, the best candidates for SR dosage forms are considered to be drugs that have good but not too great aqueous solubility.

When formulating SR dosage forms, it is also important to take into consideration that some drugs which have reasonably good to excellent aqueous solubility may present very low dissolution rates, and thus lead to dissolution-limited drug absorption (Langer and Wise 1984; Li, Robinson et al. 1987).

Another very important factor related to aqueous solubility, which is essential to consider when formulating new SR dosage forms, is that of $\mathrm{pH}$-dependent solubility. For example, drugs that are highly soluble in the acidic $\mathrm{pH}$ of the stomach but have a considerably reduced solubility at $\mathrm{pH} 5$ or higher may have a limited period of time to dissolve before reaching their main absorption site (Langer and Wise 1984). The bioavailability of such drugs may be improved by dosage form design approaches such as gastro-retention or formulation of the drug together with an acid (if the drug is a weak base) or with a base (if the drug is a weak acid) in order to make solubility higher and independent of the external environment $(\mathrm{Li}$, Robinson et al. 1987). 
Other factors affecting the solubility of a drug include crystal polymorphism and the capacity of the compound to form hydrogen bonds (Higuchi, Lau et al. 1963; Dresse, Gerard et al. 1978).

In dissolution theory, it is assumed that an aqueous diffusion layer or stagnant liquid film of thickness $h$ exists at the surface of a solid undergoing dissolution. This thickness $h$ represents a stationary layer of solvent in which the solute molecules exist in concentrations from $C_{s}$ to $C$. Beyond the static diffusion layer, at $x$ greater than $h$, mixing occurs in the solution and the drug is found at a uniform concentration, $C$, throughout the bulk phase. The dissolution of the drug can be described by the Noyes-Whitney equation:

$$
\frac{d M}{d t}=\frac{D \cdot A\left(C_{s}-C\right)}{h}
$$

where, $d M / d t$ is the rate of dissolution of the drug particles; $A$, the effective surface area of the drug particles in contact with the gastrointestinal fluids; $D$, the diffusion coefficient of the drug in solution in the gastrointestinal fluids; $h$, the thickness of the diffusion layer around each drug particle; $C_{s}$, the saturation solubility of the drug in solution; and $C$, the concentration of the drug in the gastrointestinal fluids.

The dissolution rate of a drug under sink conditions, according to the Noyes-Whitney equation, is directly proportional to its intrinsic solubility in the diffusion layer surrounding each dissolving drug particle, $C_{S}$.

Different levels of approximate solubilities have been described, and are shown in Table 1.4 (Aulton 2002). 
Table 1.4 Levels of approximate solubilities.

\begin{tabular}{|ll|}
\hline Solubility description & $\begin{array}{l}\text { Approximate weight of solvent }(\mathrm{g}) \\
\text { necessary to dissolve } 1 \mathrm{~g} \text { of solute }\end{array}$ \\
\hline Very soluble & $<1$ \\
Freely soluble & Between 1 and 10 \\
Soluble & Between 10 and 30 \\
Sparingly soluble & Between 30 and 100 \\
Slightly soluble & Between 100 and 1000 \\
Very slightly soluble & Between 1000 and 10000 \\
Practically insoluble & $>10000$ \\
\hline
\end{tabular}

Regarding the drugs used in the present study, acetaminophen solubility in water varies from sparingly soluble to soluble at $37^{\circ} \mathrm{C}$, with $\mathrm{pH}$-independent solubility at physiological values (from 1 to 8.5 ) (Forrest, Clements et al. 1982), and tramadol hydrochloride is freely soluble in water and in ethanol (http://chemicalland21.com/lifescience/phar/TRAMADOL HCl.htm, last visited on 20th December 2013).

\subsubsection{Partition coefficient and molecular size}

Following administration, the drug must penetrate a variety of membranes to gain access to the site of action. The partition coefficient and the molecular size of a drug are essential in determining its feasibility as a candidate for SR products because these properties influence both the penetration of the drug across biological membranes and its diffusion across a rate-controlling membrane or matrix in the SR system itself (Langer and Wise 1984; Li, Robinson et al. 1987).

The partition coefficient of a drug describes the ratio of the concentrations of that drug distributed between two immiscible solvents at equilibrium, and is usually expressed as concentration in oil or water-immiscible non-polar solvent/concentration in water (Langer and 
Wise 1984). Therefore, this coefficient is a measure of the lipid solubility of a drug and influences greatly its absorption characteristics. Drugs with very high partition coefficient, i.e., very oil-soluble, promptly penetrate the membranes, but will preferably remain in the lipid phase rather than pass into the blood circulation. Drugs with extreme aqueous solubility, i.e., low oil/water partition coefficients, on the other hand, can hardly penetrate the membranes. Therefore, a balanced partition coefficient is mandatory to attain optimum drug diffusion across both biological membranes and rate-controlling polymeric membranes or matrices (Langer and Wise 1984; Li, Robinson et al. 1987).

One of the water-immiscible solvents most extensively used in the prediction of drug absorption is the fatty alcohol, octanol. In this case, octanol represents the lipophilic phase because it is assumed to have a degree of lipophilicity comparable to that of a cell membrane (Artursson, Palm et al. 2001). According to octanol/water partitioning coefficient $\left(\mathrm{P}_{\text {oct }}\right)$, the permeability in the intestinal epithelium increases approximately with the lipophilicity of the drug molecule until a $\log \mathrm{P}_{\text {oct }}$ value of about two is reached, when the absorption is considered maximal in humans. A $\log \mathrm{P}_{\text {oct }}$ value $>3-4$ is associated with a decrease in permeability, given that very hydrophobic drugs usually have low aqueous solubility and will tend to pass at a slower rate from the lipophilic cell membranes to the aqueous extracellular fluids (Varma, Khandavilli et al. 2004). In the case of ionizable drugs, the apparent distribution coefficient at pH $7.4\left(D_{\text {oct }}\right)$ is often used instead of $P_{\text {oct }}$ (Artursson, Palm et al. 2001).

Drugs with large molecular sizes also produce erratic drug absorption across the intestinal membrane, which will make the design of an oral SR dosage form of that drug difficult. Therefore, in order to consider drug diffusion through a polymeric membrane or matrix as the rate controlling mechanism for a SR dosage form, the candidate drug must have a molecular size and a partition coefficient which will allow it to rapidly penetrate and diffuse through membranes (Langer and Wise 1984). 


\subsubsection{Biopharmaceutical Classification System}

For many oral dosage forms, the main parameters controlling the extent and rate of drug absorption are its aqueous solubility and gastrointestinal permeability (Amidon, Lennernas et al. 1995). Therefore, a classification system categorizing drug molecules into four classes according to their solubility along the gastrointestinal $\mathrm{pH}$ range and their permeability across the gastrointestinal membrane was proposed. This classification is presented in Table 1.5 (Amidon, Lennernas et al. 1995):

Table 1.5 Biopharmaceutical Classification System (BCS).

\begin{tabular}{|lll|}
\hline & High permeability & Low permeability \\
\hline High water solubility & Class I & Class III \\
Low water solubility & Class II & Class IV \\
\hline
\end{tabular}

A drug is considered to be highly soluble when the maximal dose strength available is soluble in $250 \mathrm{~mL}$ or less of water or a buffer over a large $\mathrm{pH}$ range (1-8), and highly permeable when the fraction of absorbed dose in humans is expected to be greater than $90 \%$ (Ashford 2002).

The BCS was introduced as a useful tool for the replacement of certain in vivo bioequivalence studies with accurate in vitro dissolution and permeability studies. These in vitro studies are expected to be capable of predicting in vivo performance of a drug (Galia, Nicolaides et al. 1998). According to this model, all drugs except the ones belonging to the Class I will lead to unreliable intestinal absorption and low bioavailability. It is desirable that candidates to SR dosage forms belong to Class I drugs, so that drug-release from the dosage form is the rate-limiting step and the drug is rapidly and reliably transported across the gastrointestinal wall. 


\subsubsection{4 $p K_{a}$ (logarithmic measure of the acid dissociation constant)}

Once in solution, the absorption of a drug is also influenced by its dissociation constant, $K_{a}$, and the $\mathrm{pH}$ at the absorption site. The $p K_{a}$ of a drug is an important physicochemical consideration in the formulation of SR dosage forms, because it indicates the fraction of the drug that will be ionized and/or unionized at different $\mathrm{pH}$ values. Acidic drugs with low $p K_{a}$ values will have a higher fraction of uncharged species in the low $\mathrm{pH}$ of the stomach and first section of the small intestine. However, they may be nearly completely ionized in the mid to lower small intestine where the $\mathrm{pH}$ increases to 7 and above. Basic drugs, on the other hand, have higher $p K_{a}$ values and will be more soluble in the acidic $\mathrm{pH}$ of the stomach, where they are mostly ionized, but may be almost entirely in their uncharged form under the conditions of the higher $\mathrm{pH}$ in the mid to lower small intestine. Most drugs are preferentially absorbed in their unionized form, as only this form can diffuse readily across the

cell membranes. Therefore, the $p K_{a}$ of a drug provides a good indication of the main site of absorption of the drug along the gastrointestinal tract as well as indicate possible problems with the local absorbability (Langer and Wise 1984).

The extent to which a weakly acidic or basic drug ionizes in solution in the gastrointestinal fluid can be calculated using the appropriate form of the HendersonHasselbalch equation. For example, for a weakly basic drug possessing a single ionizable group the equation takes the form of:

$$
\log \frac{\left[B H^{+}\right]}{[B]}=p K_{a}-p H
$$

Equation 12

\subsubsection{Drug stability}

As described before, regardless of the type of dosage form employed, an orally administered drug will be exposed to the luminal contents of the gastrointestinal tract $(\mathrm{pH}$ range, ionic environment, enzymes and flora) before it is absorbed. For this reason, the 
stability of a drug in the gastrointestinal environment is another decisive physicochemical factor to be considered when determining the viability of designing a SR dosage form containing that drug, or when considering dosage forms design approaches (Langer and Wise 1984; Li, Robinson et al. 1987). By gradually releasing the drug to solution in an environment in which it is unstable, a SR dosage form will cause the destruction of just about the entire drug amount. For example, drugs that undergo extensive metabolism along the intestinal tract and in the gastrointestinal membrane may be poor candidates for SR dosage forms. Releasing such drugs in low concentrations as they transit along the small intestine will increase the fraction of the drug that is metabolized (Langer and Wise 1984). In addition, if some degree of colonic absorption is expected, stability to the metabolizing effect of the colonic bacterial population is also required (Gupta and Robinson 1992). Typically, the drug must be stable in the $\mathrm{pH}$ range of 1 to 8 to be considered viable in SR systems (Gupta and Robinson 1992).

Regarding the drugs used in the present study, the first drug, acetaminophen, has $\mathrm{pH}$ independent solubility at physiological values (from 1 to 8.5). Hence, this drug is not considered to ionize in the physiological pH range (Forrest, Clements et al. 1982). Tramadol hydrochloride, the second model drug, has been found to be stable under extreme conditions of $\mathrm{pH}(1 \mathrm{~N}-\mathrm{HCl}$ and $1 \mathrm{~N}-\mathrm{NaOH})$ for at least 4 weeks (Zaghloul and Radwan 1997).

Acetaminophen is not subject to any significant enzymatic or bacterial degradation in the gastrointestinal lumen (Mattok and McGilveray 1973). The fact that after oral administration, nearly all tramadol hydrochloride is absorbed from the gastrointestinal tract (Grond and Sablotzki 2004), suggests that this drug is also stable in the gastrointestinal tract fluids. Although, the bioavailability of tramadol after a single administration is $70 \%$ due to extensive first-pass metabolism (Malonne, Sonet et al. 2004), the bioavailability of a SR dosage form at steady state is $100 \%$ relative to an IR formulation (Raber, Schulz et al. 1999). 


\subsubsection{Biological and pharmacological properties of drugs influencing their viability as candidates for sustained drug-release dosage forms}

\subsubsection{Size of dose, biological half-life and duration of action}

The size of a drug dose is a major factor determining the feasibility of a candidate drug for oral SR. When considering the dose of drug to be incorporated into a SR dosage form, it is as well necessary to take into consideration the biologic or elimination half-life of that drug (generally referred to as $t_{1 / 2}$ ), as these two aspects are related. The $t_{1 / 2}$ is the period of time required for any specific concentration of a drug in the body to decrease by one-half following completion of the absorption phase. It gives an index of the residence time of that drug in the body as well as of its duration of action. Therefore, $t_{1 / 2}$ also plays a key role in the process of deliberating if a drug is suitable for oral SR dosage forms. Factors influencing the $t_{1 / 2}$ of a drug include its elimination, metabolism, and distribution patterns (Langer and Wise 1984; Li, Robinson et al. 1987; Gupta and Robinson 1992).

Drugs with very short $t_{1 / 2}$ require frequent dosing in order to minimize fluctuations in blood levels accompanying conventional oral dosage regimens. Therefore, it would appear that SR is an advantageous approach for delivering such drugs. However, the formulation of SR dosage forms containing these drugs poses some difficulties. First, their elimination rate is quite high, and for the required period of time during which the drug is to be released in order to achieve the desired duration and intensity of effect, this high elimination rate may, in turn, lead to a prohibitively large total dosage for the SR product, especially when the usual conventional single dose is already relatively big. This large quantity of drug added to the quantity of excipients required to retard its release may exceed that which can be easily swallowed in a single solid dosage form. This situation applies particularly to drugs with an $t_{1 / 2}$ of less than 2 hours, as well as those that are administered in large doses (Gupta and Robinson 1992; Kumar, Bhowmik et al. 2010). Besides, the quantity of polymer necessary to efficiently retard the release of drug from matrix dosage forms often equals or surpasses the quantity of drug being retarded. Moreover, it is mandatory to develop a "fail-safe" dosage form that will not allow dumping of all the dose in the product at one time caused by failure of 
the dosage form or by exposure to a combination of physiological factors (Langer and Wise 1984), in particular for high dosages and/or drugs with a narrow therapeutic window.

Drugs with long elimination half-lives of eight hours or more are, on the other hand, commonly sufficiently sustained in the body when administered in conventional dosage forms. Hence, SR dosage forms are generally not necessary in such cases (Gupta and Robinson 1992; Kumar, Bhowmik et al. 2010).

Therefore, the best candidates for SR systems are usually drugs with relatively short, but not very short, biological half-lives, which require frequent administration of a conventional dosage form to provide therapeutic drug levels (Langer and Wise 1984).

Relatively to the drugs used in the present work, the reported values for the plasma mean $t_{1 / 2}$ of acetaminophen, the first model drugs used, are about 1 to 4 hours (Cao, Choi et al. 2005). Therefore, this drug was replaced by tramadol hydrochloride, which has a mean $t_{1 / 2}$ of about 5 to 6 hours (Raffa, Nayak et al. 1995).

\subsubsection{Absorption properties}

As a rule, for SR formulations, the rate-limiting step is the rate of drug-release from the dosage form. Therefore, it is desirable that drug absorption from the gastrointestinal tract be faster than drug-release from the dosage form. For this reason, efficient drug absorption is a requirement for a drug to be considered as candidate for oral SR applications (Gupta and Robinson 1992).

Although, it would be preferable to have a complete absorption of the released drug, variation in both the extent and rate of drug absorption can take place due to the multiplicity of factors already described in a previous section, such as hydrolytic degradation by digestive enzymes, physical loss, metabolism by the intestinal flora or during transit across the gastrointestinal membrane. In addition, the diverse absorptive capacity of the different segments of the gastrointestinal tract can also influence the amount and rate of absorption for some drugs. However, as long as the variations in drug absorption are not very significant, a successful SR dosage form can be produced (Li, Robinson et al. 1987). 
For drugs that are absorbed by passive diffusion, as it is the case of the model drugs used in the present study, the permeation across the gastrointestinal membrane usually shows a consistent pattern, even though the rate of drug absorption may decline progressively. But, for drugs that are absorbed by means of specialized transport mechanisms, such as active transport, and/or at restricted areas along the gastrointestinal tract, absorption from the gastrointestinal lumen may not be consistent, which limits their suitability for SR applications (Langer and Wise 1984; Li, Robinson et al. 1987; Gupta and Robinson 1992).

Another important aspect of the absorption process via the oral route is the magnitude of the absorption rate constant. Drugs with very low absorption rate constants are poor candidates for SR products because the desired release rate constant from the dosage form would have to be even lower. Besides, as the gastrointestinal transit time is restricted, a SR dosage form containing a drug with a very low absorption rate constant will generally have poor drug bioavailability (Li, Robinson et al. 1987).

In literature, it is stated that following oral administration tramadol is rapidly and almost completely absorbed by passive diffusion. Its bioavailability of $70 \%$ after a single administration can be attributed entirely to extensive first pass metabolism (Grond and Sablotzki 2004; Malonne, Sonet et al. 2004; Mattia and Coluzzi 2005). Acetaminophen is also rapidly absorbed by passive diffusion, its systemic bioavailability being dose-dependent and ranging from 70 to $90 \%$. Its rate of oral absorption is predominantly dependent on the rate of gastric emptying and on the factors and drugs that influence it. Acetaminophen is also well absorbed from the rectum (Forrest, Clements et al. 1982).

\subsubsection{Distribution}

Although having as much information as possible on the disposition of drugs within the body would be advantageous for the development of new SR products, usually, decisions are made using only a few pharmacokinetic parameters as a guide (Li, Robinson et al. 1987). One of these pharmacokinetic parameters is the apparent volume of distribution of a drug, $V_{\mathrm{d}}$, which is frequently used to describe the magnitude of distribution, including binding to various tissues and plasma proteins in the body. However, this parameter does not have a 
precise physiological meaning. Conceptually, $V_{\mathrm{d}}$ is rather considered a proportionality constant relating the drug concentration in the plasma to the total amount of drug in the body, either circulating in the blood or in target tissues (Li, Robinson et al. 1987; Shargel, Wu-Pong et al. 2004).

The distribution of a drug to the diverse tissues in the body and its binding to plasma proteins can influence the duration of action of that drug. This is because the fraction of drug and/or active metabolite(s) that is bound can be considered to be inactive and unable to cross further membranes to reach sites of action and elimination organs (Langer and Wise 1984; Li, Robinson et al. 1987). Accordingly, the extent and rate at which the drug is bound, and the extent and rate at which is subsequently unbound will affect the duration of drug action, even when the rate of elimination of the unbound fraction is relatively rapid (Langer and Wise 1984). At high binding, prolonged drug action may take place (Langer and Wise 1984; Li, Robinson et al. 1987).

Tramadol is rapidly distributed in the body and has a plasma protein binding of about $20 \%$ (Grond and Sablotzki 2004). The volume of distribution of tramadol has been reported to be $2.7 \mathrm{~L} / \mathrm{kg}$ in humans (Lewis and Han 1997). Acetaminophen also distributes rapidly and evenly throughout most tissues and fluids and has a volume of distribution of approximately $0.9 \mathrm{~L} / \mathrm{kg}$. The fraction of the drug bound to red blood cells is 10 to $20 \%$ (Forrest, Clements et al. 1982; Morris and Levy 1984).

\subsubsection{Metabolism}

Metabolism can either transform active drugs into inactive metabolites or inactive drugs into active metabolites.

Some drugs are significantly restricted from SR product design because of their metabolism. First, when the drug is metabolized during its passage through the intestinal membrane or through the liver, dose-dependent bioavailability generally occurs. Since both intestinal and hepatic metabolism are saturable processes, the fraction of drug lost due to metabolism is dose-dependant and a significant reduction in bioavailability would be predictable if a drug is gradually released over a prolonged period of time. However, the 
design of oral SR dosage forms for drugs that are widely metabolized is possible, even though difficult, as long as the rate and extent of metabolism are predictable and the rate constants for the process are not too large (Li, Robinson et al. 1987).

Tramadol is also widely metabolized in the liver with a first-pass effect of up to $30 \%$, its main metabolite being O-desmethyltramadol (Grond and Sablotzki 2004). However, both tramadol enantiomers and their metabolites contribute to the analgesic activity by means of different mechanisms (Raffa, Friderichs et al. 1992). Acetaminophen is extensively metabolised, predominantly in the liver, the major metabolites being the sulphate and glucuronide conjugates (Forrest, Clements et al. 1982). The metabolism of both drugs was detailed in section 1.4.3.4.

\subsubsection{Safety margin and side effects of the drug}

A key aspect of the choice of drug candidates for SR products is their safety and side effects in the administered doses. For some drugs, the incidence of side effects can be minimized by controlling the plasma drug concentration for the duration of the therapy, and thus SR dosage forms can offer a solution to this problem.

Tramadol, one of the drugs used in the present work, is an example of a drug whose toxic effects can be reduced by the use of SR dosage forms. Indeed, the lower incidence of adverse effects with SR tramadol has been demonstrated, presumably because high peak concentrations did not occur (Hummel, Roscher et al. 1996; Radbruch, Grond et al. 1996).

The therapeutic index, as defined by the following equation, has been widely used as a crude estimation of the relative margin of safety of a drug and its balance safety-efficacy:

Therapeutic index $=\mathrm{TD}_{50} / \mathrm{ED}_{50}$

Equation 13

where $\mathrm{TD}_{50}$ is the toxic dose of drug for $50 \%$ of the population and $\mathrm{ED}_{50}$ is the minimum effective dose for $50 \%$ of the population. 
SR dosage forms containing drugs with narrow therapeutic indices must generate a precise and reproducible drug-release pattern in order to attain plasma concentrations within the therapeutically safe and effective window. Besides, it is necessary to take into consideration other factors which can potentially influence the plasma drug concentrations attained, such as patient physiological variability, drug accumulation upon multiple dosing, concurrent disease states or eventual drug interactions (Langer and Wise 1984; Li, Robinson et al. 1987). All these factors can make the design of SR products for drugs with narrow therapeutic indices difficult. Yet, when properly prepared, oral SR formulations are valuable for maintaining plasma levels within a narrow therapeutic window, even for drugs with a narrow therapeutic index such as tramadol (Overholser and Foster 2011), the second model drug of this study, or theophylline (Glynn-Barnhart, Hill et al. 1988; Devarajan, Sule et al. 1999).

\subsubsection{Therapeutic goal, disease state and circadian rhythm}

When considering the design of a new SR dosage form for a particular drug, the desired outcome of the therapy should be defined, as in certain health conditions this type of products may not be the desirable or rational choice. For example, oral SR dosage forms are contraindicated for antibiotics whose effectiveness is related to high spike blood levels on repeated administration, or in situations where a rapid onset of action is expected, such as a cardiac emergency, an asthmatic attack or an angina attack. However, SR products may be useful in the prevention of some of these situations as, for example, angina pectoris (Langer and Wise 1984).

While disease state and circadian rhythm are not drug properties, in some cases, they are just as important as drug properties when considering a drug for SR (Li, Robinson et al. 1987). Indeed, some physiological processes and disease states follow circadian rhythms, reaching peak or valley effects at specific times of the day. Examples are blood pressure, which is characterized by a nocturnal fall and a diurnal rise (Imai, Abe et al. 1990) and asthma episodes, which occur most often at night-time or early-morning for most asthmatics (Goldenheim, Conrad et al. 1987). SR products may be the best treatment approach for these 
conditions, since they can better assure maintenance of prophylactic blood or tissue concentrations, and thus be a better choice in their prevention or control.

Maintenance of some prophylactic blood or tissue levels may be also required to control symptoms of a disease, such as pain caused by certain forms of cancer or rheumatoid arthritis, which can be controlled by oral administration of tramadol SR formulations.

\subsection{Pharmacokinetic considerations and analysis in the design of dosage forms}

\subsubsection{Importance of biopharmaceutics, pharmacokinetics and pharmacodynamics}

When considering strategies for the design of a new oral dosage form for a particular drug, it is essential to take into account the sequence of events that precedes or concurs the induction of a pharmacologic response upon reaching minimum effective drug concentrations at the site(s) of action. These events include administration, drug-release from the dosage form, absorption of the drug molecules from the absorption site to the blood, distribution of drug molecules from blood to tissues, and elimination via metabolism and/or via the passage of drug molecules from the blood to the exterior of the body through urine, bile or other routes. The physicochemical properties (and interrelated biological properties) of a drug, the dosage form used to administer it and the route of administration significantly influence this sequence of events, and are thus decisive determinants of the in vivo performance, safety and therapeutic efficacy of that drug.

Biopharmaceutical, pharmacokinetic and pharmacodynamic studies of drugs and drug products are usually carried out to understand this interrelationship between the physicochemical properties of the drug, the route of administration, the dosage form in which the drug is administrated and the pharmacological effect produced by the drug. 
Biopharmaceutics is essentially related to all the factors that are fundamental to ensure that the drug is delivered to the correct part of the body, in the right concentration and at the right rate, i.e., the aspects that determine its bioavailability. Therefore, biopharmaceutics includes the study of the factors that influence the stability of the drug in the dosage form and in the gastrointestinal environment, the release of the drug from the dosage form, the rate of dissolution of the drug, and its absorption into the systemic circulation.

Pharmacokinetics, usually abbreviated as $P K$, is the study of the time course of drug absorption, distribution, metabolism and excretion (Shargel, Wu-Pong et al. 2004). It describes the drug concentration-time courses in the body fluids, resulting from administration of a specific dose of the drug being studied (Meibohm and Derendorf 1997). Pharmacokinetics allows the description in quantitative terms of not only the kinetics of the active drug but also of its metabolites. The processes of drug absorption, distribution, metabolism and excretion are often abbreviated in pharmacokinetics as $A D M E$, or $\angle A D M E$ if liberation from the dosage form is also to consider (Shargel, Wu-Pong et al. 2004). In addition, the description of drug distribution and elimination is often termed drug disposition.

Another important element for understanding the factors inducing a pharmacological or toxicological response is pharmacodynamics. This discipline, which can be referred to as $P D$, studies the relationship between a certain drug concentration and the response obtained. As the same dose of drug can result in different concentrations, it is important to study this link between drug concentrations at the site of action (receptor), if possible, or in the blood plasma or serum and the pharmacological or toxicological effects.

Finally, pharmacokinetic/pharmacodynamic ( $P K / P D)$ modeling builds the bridge between the concentration-time profile resulting from the administration of a certain dose, as assessed by pharmacokinetics, and the intensity of the observed response, as quantified by pharmacodynamics (Derendorf, Lesko et al. 2000). Thus, the resulting so-called integrated $P K / P D$-models allow the establishment and evaluation of dose-concentration-response relationships, and describe the effect-time courses resulting from a drug dose (Meibohm and Derendorf 1997; Derendorf, Lesko et al. 2000). 


\subsubsection{Experimental approaches of pharmacokinetics}

\subsubsection{Measurement of drug concentrations}

The measurement of the amounts or concentrations of drugs and metabolites in body fluids or tissues at different times after the administration of a dosage form gives much information on drug absorption, distribution and elimination and is, therefore, a fundamental element in the determination of individual or population pharmacokinetics. Although, concentrations can be measured from different body fluids, e.g., plasma, urine, saliva and milk, or even from biological tissues, the biological samples most frequently used for assessing the pharmacokinetics of a drug and its metabolite(s) in the body are concentration (levels) in blood plasma or serum. Urine may also be sampled to assess the drug amounts excreted unchanged.

The main components of whole blood are red blood cells, white blood cells, and platelets (which release the clothing factors), suspended in plasma (which contains proteins, such as albumin and fibrinogen, hormones, minerals and vitamins, among other constituents). Serum is prepared by obtaining a blood sample, followed by blood clot formation and removal of the clot using a centrifuge to obtain the supernatant. To obtain plasma, an anticoagulant, such as heparin, is added to the blood sample to prevent the clot formation, and then the plasma is obtained from the supernatant after centrifugation. Therefore, the main difference between the two lies in their protein content. Plasma still contains clotting factors, whereas serum has had the clothing factors removed.

The number of blood samples should be large enough and the timing appropriate to allow an adequate determination of the absorption, distribution and elimination phases.

Ideally, the target tissue should be sampled and the concentrations of drug that reach it measured instead of plasma concentrations. However, this is impractical in most situations. Therefore, as plasma perfuses all the tissues of the body and assuming that there is a dynamic equilibrium between drug in the plasma and drug in the tissues, then, changes in plasma drug concentrations are considered to reflect changes in tissue drug concentrations (Shargel, WuPong et al. 2004). 
Since very important decisions in drug development and dosage form development are based on diverse studies, including bioavailability, pharmacokinetic and pharmacodynamic studies using data obtained from analytical results, it is imperative that the analytical methods for the measurement of drugs and metabolites be selective, accurate, and sensitive (Braggio, Barnaby et al. 1996). As a rule, these measurements in biological materials are validated so that accurate and reliable information is generated. Among the various methods that permit drugs and their metabolites to be separated, indentified and quantitatively assayed, chromatography is the most frequently employed (Hirtz 1986), in particular liquid chromatography (LC) coupled to different detectors such as mass spectrometer (MS) or UV detector. Indeed, the LC-MS methods are considered as most appropriate for determination of drugs and their metabolites and are also best suited for high throughput analysis (Roškar and Lušin 2012).

In the first article of this research (Nabais, Brouillet et al. 2007), acetaminophen plasma concentrations were analyzed by high-performance liquid chromatography (HPLC) coupled to UV-Vis spectrophotometric detector. The development and validation of LC/MS methods for quantification of tramadol, the second drug used in this study, and for its active metabolite O-desmethyltramadol in human plasma have also been described and successfully applied to both pharmacokinetic and bioequivalence studies (Vlase, Leucuta et al. 2008; Patel, Sharma et al. 2009).

\subsubsection{Plasma drug concentrations as a function of time}

The most important source of information in pharmacokinetics is the plasma drug concentration versus time curve. The pharmacokinetic parameters, which pharmacokinetic studies are based on, are derived from these curves.

A description of the plasma concentration versus time curves obtained after administration of an oral dosage form and after administration of an intravenous bolus, the two forms of administration used in the in vivo studies performed in the scope of the present thesis, is given next. 
Plasma concentration-time curve following a single oral dose

The typical plasma concentration-time curve following a single oral dose of a drug administered in a IR dosage form is characterized by a gradual rise up to a maximum as the drug reaches the systemic circulation, usually called absorption phase, followed by a gradual decline due to the removal of drug by distribution and elimination, usually called elimination phase.

Initially, the rate of drug absorption exceeds the rate at which the drug is being removed from the systemic circulation. As a result, the segment of the curve corresponding to the absorption phase is more steep that the segment corresponding to the elimination phase. However, the elimination of a drug initiates as soon as it appears in the plasma. At the peak (the highest concentration of drug achieved in plasma), the rate of drug absorption equals the rate of drug removal. During the first segment of the elimination phase, the rate of drug removal from blood becomes more rapid than the rate of absorption because the amount of solubilized drug existing at the absorption site declines progressively. However, here, the processes of absorption into the systemic circulation, distribution into all the tissues within the body, and elimination by excretion (through urine, bile or other routes), metabolism or a combination of both, happen simultaneously. After the end of absorption, the rate of drug elimination continues to decline until complete elimination from the body. 


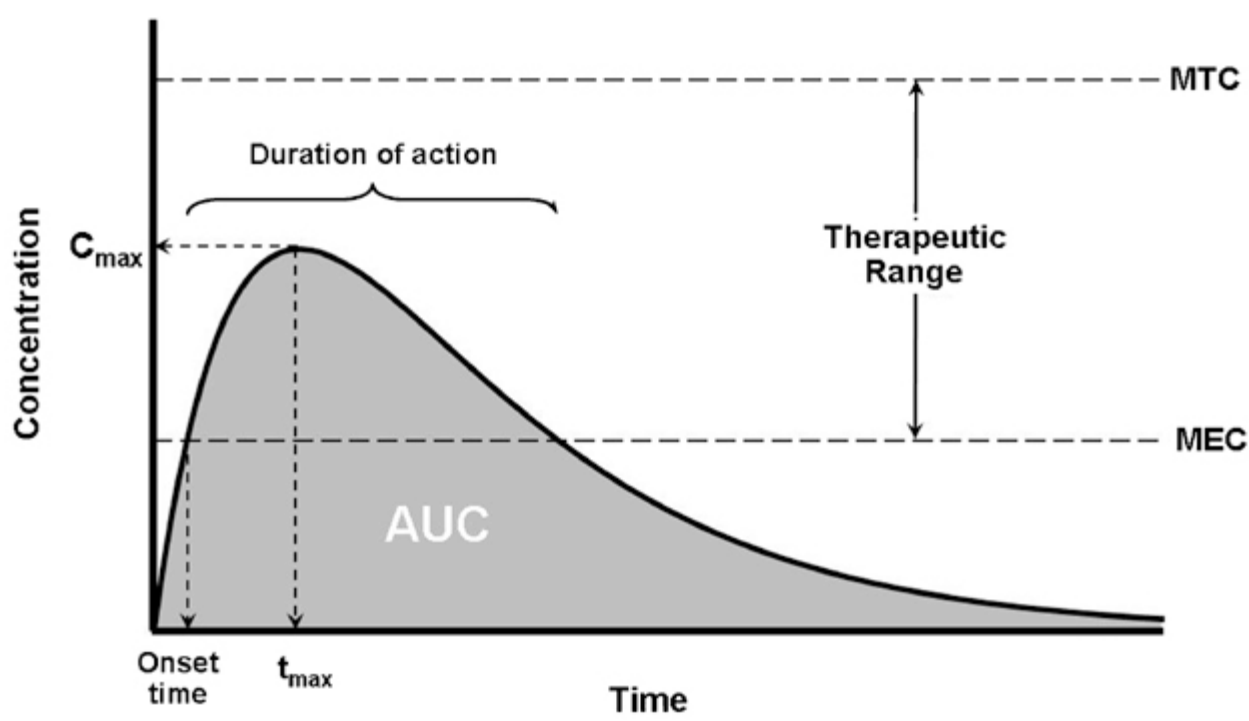

Figure 1.24 Typical plasma drug concentration versus time after oral administration of a conventional dosage form, representing the maximum concentration in plasma, $C_{\max }$, the time to reach the maximum concentration, $T_{\max }$, the therapeutic range, the MTC and the MEC, the onset time and the duration of action.

The already mentioned maximum drug concentration in the plasma or peak concentration $\left(C_{\max }\right)$ and the period of time required to achieve that same peak concentration of drug after administration of a single dose $\left(T_{\max }\right)$ (Figure 1.24) are two pharmacokinetic parameters that can be directly obtained from the curve. The area under the plasma concentration-time curve, usually known as AUC (Figure 1.24), a parameter calculated using drug concentration-time data from the curve is also shown. These pharmacokinetic parameters will be described in a following section. Other important information that can be obtained directly from the curve is the onset time, that is, the time required to achieve the minimum concentration following administration, and the duration of drug action (Figure 1.24), which represents the difference between the onset time and the time for the drug to decline back to the minimum effective concentration; in other words, the period of time during which the concentration of drug in the plasma surpasses the minimum effective concentration. 


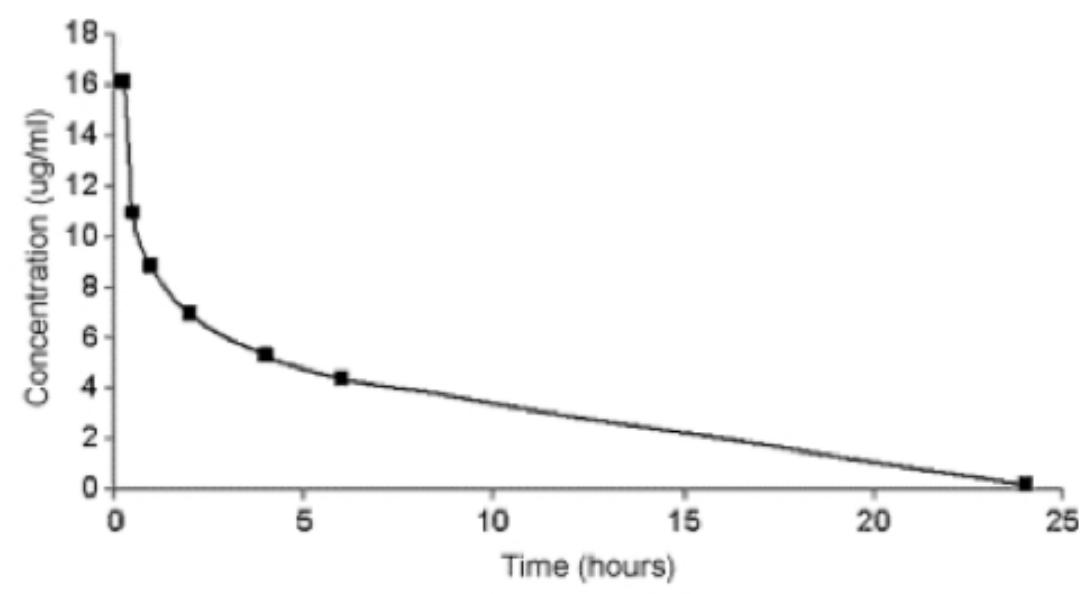

Figure 1.25 Plasma concentration versus time profile of acetaminophen in one volunteer after an intravenous single dose. Figure reproduced from (Ing-Lorenzini, Desmeules et al. 2009) with permission of Elsevier Ltd.

As explained before, when a drug is delivered by an intravenous bolus, the entire administered dose is introduced directly into the systemic circulation, and thus it does not have to pass any absorption barriers. Therefore, the dose is considered to be totally bioavailable. The maximum plasma concentration is achieved at time zero and is followed only by the gradual decline attributed to the removal of drug from the plasma by distribution and elimination (Figure 1.25). 


\subsubsection{Theoretical aspects of pharmacokinetics}

\subsubsection{Development of pharmacokinetic models}

Living organisms are complex biologic systems in which it is difficult to establish quantitative relationships between a specific drug dose, the route of administration used to give it, the concentration of drug in different anatomical locations, the pharmacological effect achieved and the elapsed time. With the aim of obtaining an adequate description of the time course of drug concentrations within the body, different mathematical methods were proposed. These methods include compartmental pharmacokinetic analysis, physiological models and non-compartmental models based on statistical moment theory. Within the scope of the research performed in this work, only pharmacokinetic compartmental analysis was used and, therefore, it will be the only methodology described herein.

In order to develop a pharmacokinetic model, simplifying hypotheses regarding the movement of the drugs within the body are made. The hypotheses or 'models' are conceived using mathematical terms, which describe quantitative relationships concisely. These mathematical models make it possible to develop differential equations that express the rates of the processes of absorption, distribution and elimination, and allow obtaining equations that describe and predict the quantity and concentration of drug in the body in function of time (Shargel, Wu-Pong et al. 2004). To develop these equations, a set of independent and dependent variables, known as experimental data, are needed. Usually, the independent variable is time and the dependent variable is the plasma drug concentration described above.

A mathematical equation relates an independent variable to a dependent variable often through the use of pharmacokinetic parameters. Thereby, the concentration-time relationship is defined by pharmacokinetic parameters. A pharmacokinetic parameter is a constant for the drug, which is estimated by fitting the equation to the experimental data. In other words, a mathematical procedure called 'fitting', is used to find the best curve fitting to a given set of experimental concentration-time data through an equation that describes that curve and which incorporates the pharmacokinetic parameters. The best fitting of that equation to the data will allow the estimation of the parameters, which will define the model. Computer programs are commonly used to facilitate curve fitting and parameter estimation. The model equation is 
then used to predict plasma drug concentrations versus time. Various mathematical models can be created to simulate the rate processes of drug absorption, distribution, and elimination.

Pharmacokinetic models can be used to evaluate differences in bioavailability between equivalent dosage forms (relative bioavailability and bioequivalence), to compare the bioavailability resulting from different routes of administration (absolute bioavailability), or from different dosage forms. In the in vivo studies carried out in this research, pharmacokinetics was used to compare a SR dosage form and an IR dosage form. Besides, pharmacokinetic models are used to predict plasma, tissue, and urine drug concentrations with any dosage regimen, correlate drug concentrations with pharmacologic or toxicologic activity, describe how changes in physiology or disease affect the absorption, distribution, or elimination of the drug, explain drugs interactions, evaluate food effects in single or multiple dose administration, among other applications (Urso, Blardi et al. 2002; Shargel, Wu-Pong et al. 2004).

\subsubsection{Compartmental pharmacokinetic models}

Compartmental models provide a very simplified kinetic approach to describe drug absorption, distribution, and elimination. These models group by affinity different anatomical and physiological areas of the body into one or more compartments. These open compartments communicate reversibly with each other, i.e., a particular amount or concentration of drug is exchanged between two compartments per unit time, including to and from the plasma compartment, also called central compartment.

A compartment is not a real physiologic or anatomic region but is considered as a tissue or group of tissues or organs with similar kinetic properties, i.e., with similar blood flow and drug affinity. Within each compartment it is assumed that the drug is rapidly and homogeneously distributed. Thereby, the drug concentration represents an average concentration, and each drug molecule has an equal probability of leaving the compartment. Rate constants are used to represent the overall rate processes of drug entry into and exit from the compartment. Compartment models are based on linear assumptions using linear differential equations (Shargel, Wu-Pong et al. 2004). 
Although compartment models are usually regarded as somewhat empirical and lacking physiologic relevance, they present the advantage of permitting a description of the time course of drug within the body with a limited amount of data (Shargel, Wu-Pong et al. 2004).

The simplest compartment model is the one-compartment model (Figure 1.26), which considers the drug to be distributed instantaneously and uniformly into a unique compartment in the body, usually called the central compartment or plasma compartment, from where the drug is eliminated. The central compartment represents the blood, extracellular fluid and highly perfused tissues that rapidly equilibrate with drug, such as the organs involved in drug elimination. However, drugs that exhibit a slow equilibration with peripheral tissues are best described with two-, three- (Figure 1.26) or multi-compartment models. In a two-compartment model, drug can move between the central to and from the so-called tissue or peripheral compartment, which represents the tissues in which the drug equilibrates more slowly. The more compartments the model has, the more different equilibrium rates between tissues are considered. Drug tissue concentration is assumed to be uniform within a given compartment. The compartmental models are particularly useful when little information is known about the tissues (Shargel, Wu-Pong et al. 2004). 


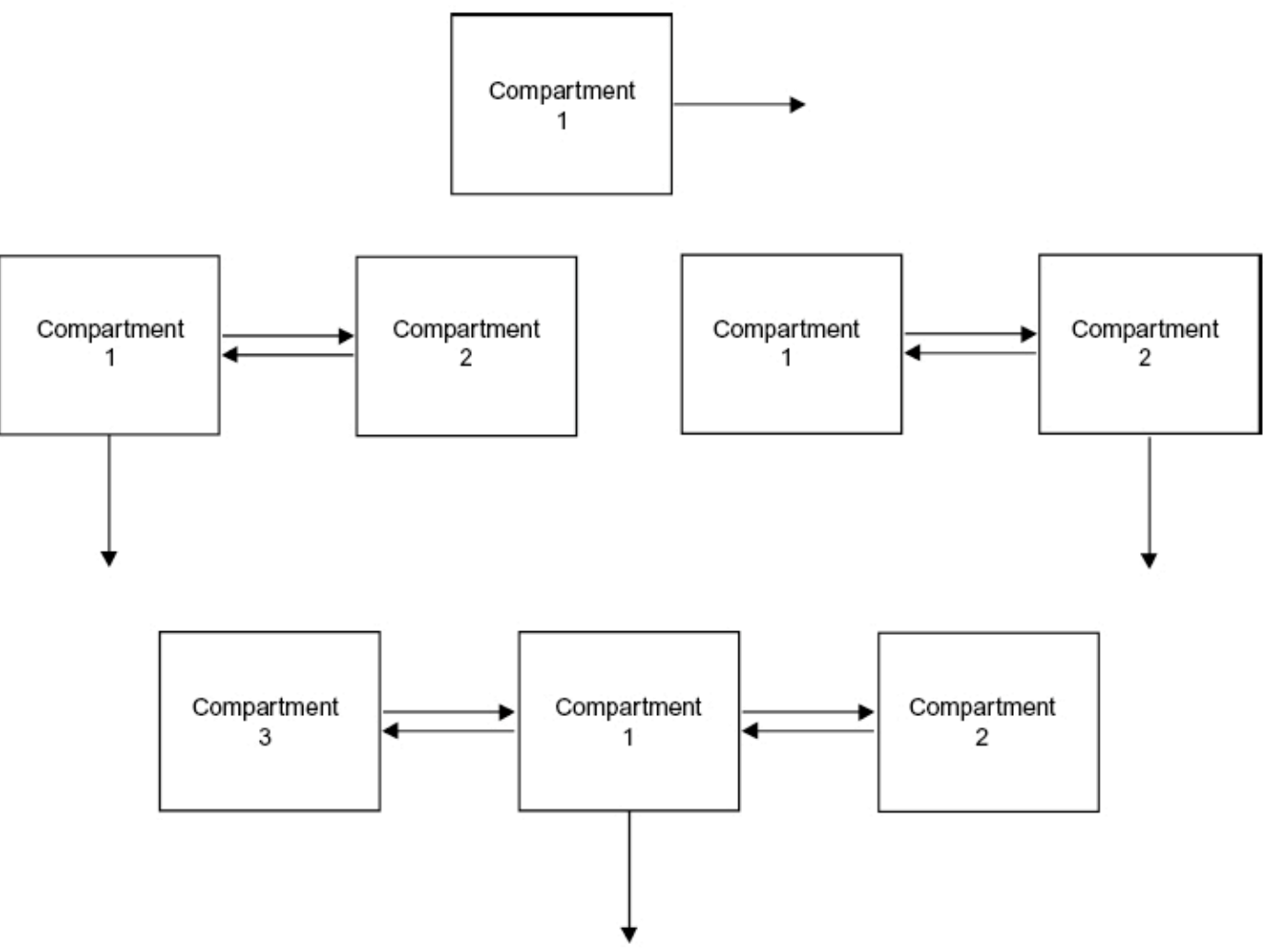

Figure 1.26 Schematic representations of one-, two-, and three-compartment models.

\subsubsection{Pharmacokinetic parameters}

As mentioned above, only through pharmacokinetic models it is possible to define a set of pharmacokinetic parameters that give a simplified description of drug absorption, distribution and elimination.

The first step in the analysis of pharmacokinetic data is usually the estimation of $C_{\max }$, $T_{\max }$, and AUC, which were described above. The $t_{1 / 2}$ may also be estimated. These four parameters can well represent the data without the need of any complex mathematical model (Urso, Blardi et al. 2002). As described before, the $t_{1 / 2}$ is the period of time required for the amount or concentration of a drug to decrease by one-half. This parameter is used to describe the decay of the drug concentration in the terminal phase and may be determined by means of the following equation: 


$$
t_{1 / 2}=\frac{\ln 2}{k}
$$

Equation 14

The $C_{\max }$ is related to the dose, the absorption rate constant $\left(k_{\mathrm{a}}\right)$, and the elimination rate constant of the drug $(k) . C_{\max }$ provides indications that the systemic drug absorption was sufficient to provide a therapeutic response or that toxic concentration may have been achieved. Although $C_{\max }$ is not a unit for rate, it can be used as a surrogate measure for the rate of drug bioavailability (Shargel, Wu-Pong et al. 2004). The $T_{\max }$ is a rough indicator of the average rate of drug absorption and can also be used to assess that rate (Ashford 2002; Shargel, Wu-Pong et al. 2004). The AUC is a parameter related to the total amount of drug absorbed into the systemic circulation following the administration of a single dose and to the ability that the system has to eliminate the drug (clearance). Changes in the AUC reflect changes in the total amount of drug absorbed and/or modifications in the kinetics of distribution, metabolism and excretion (Ashford 2002). Therefore, this parameter can be used to measure the drug amount absorbed and the efficiency of physiological processes that characterize the drug elimination (Urso, Blardi et al. 2002).

The AUC can be calculated using the trapezoidal rule (Figure 1.27). This simple numerical method considers the area between time intervals as the area of a trapezoid and uses the following equation to calculate it:

$$
[A U C]_{t_{n}}^{t_{n+1}}=\frac{C_{n}+C_{n+1}}{2}\left(t_{n+1}-t_{n}\right)
$$

Equation 15

where $t_{n}$ represents the time of observation of drug concentration, $C_{n}$, and $t_{n+1}$ is the time corresponding to the following observed drug concentration, $C_{n+1}$.

To obtain the total AUC all individual areas between two consecutive time intervals are summed. The residual AUC between the last measurable concentration and the complete removal of drug from the plasma can be calculated by extrapolation to time equal to infinity $(\infty)$, as follows: 


$$
[A U C]_{t_{n+1}}^{\infty}=\frac{C_{f}}{k}
$$

where $C_{f}$ is the last observed plasma concentration at $t_{\mathrm{n}+1}$ and $k$ is the slope obtained from the terminal portion of the curve and represents the overall drug elimination first-order rate constant.

Therefore, the full trapezoidal rule to calculate the AUC from time 0 to complete removal of drug can be expressed as follows:

$$
[A U C]_{0}^{\infty}=\sum[A U C]_{t_{n}}^{t_{n+1}}+\frac{C_{f}}{k}
$$

Equation 17

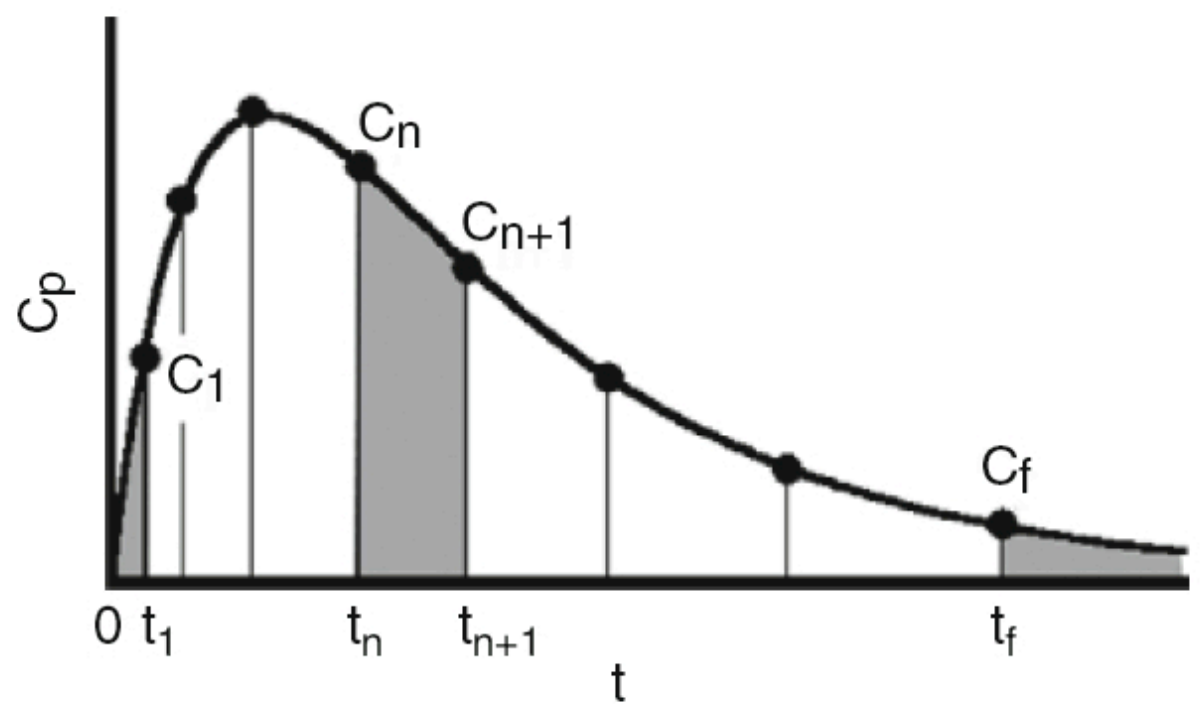

Figure 1.27 Graphical illustration of the application of the trapezoidal rule to numerically estimate the AUC for first-order absorption data. Figure reproduced from (Byers and Sarver 2009) with permission of Elsevier Ltd. 
After oral administration, $C_{\max }$ and $T_{\max }$ are dependent on the extent and rate of drug absorption and on the disposition profile of the drug. Consequently, they may characterize the properties of different formulations in the same subject (Urso and Aarons 1983).

The $C_{\max }, T_{\max }$, and AUC were determined in the in vivo studies performed in this research and used to compare a HASCA SD SR formulation and a commercialized IR one.

There are other pharmacokinetic parameters which may be important in pharmacokinetic studies, such as the apparent volume of distribution $\left(V_{\mathrm{d}}\right)$, a parameter essential to estimate the amount of drug in the body from a certain concentration of drug, and the clearance of drug elimination from the body (CL), which can be easily defined using compartmental models. However, these parameters are out of the scope of this work.

1.6.3.4 Estimation of the cumulative relative fraction of drug absorbed after oral absorption

Pharmacokinetic models also provide an easy way to obtain an estimate of the cumulative relative fraction of drug absorbed versus time after a single oral dose of a drug. This estimation was part of the treatment of the in vivo data made in the present research, and was obtained using an equation proposed by Wagner-Nelson (Wagner and Nelson 1963; Wagner and Nelson 1964):

$$
\frac{A b}{A b^{\infty}}=\frac{C_{p}+k[A U C]_{0}^{t}}{k[A U C]_{0}^{\infty}}
$$

Equation 18

where $C_{p}$ is the plasma concentration at time $t ; A b$ is the percentage of drug absorbed at time $t$ and $A b^{\infty}$ is the total amount of drug absorbed. 


\subsubsection{Statistics in pharmacokinetic studies}

Statistics are used to obtain a valid analysis of experimental data. The objective of data analysis is to obtain as much information about the population as possible based on the sample data collected.

All measurements have some degree of error. An error is the difference between the true value and the observed value. Errors in measurements may be determinate (systematic) or indeterminate (random, accidental). Determinate errors are known and controllable errors, such as instrumental errors or personal errors. These errors may be minimized in analytical procedures by using properly calibrated instrumentation, standardized chemicals, and appropriate blanks and control samples, and a more precise manipulation. Indeterminate errors are more difficult to control and are, in some cases, unknown errors (Shargel, Wu-Pong et al. 2004). Examples are limitations of reading balances and electrical "noise" in instruments. In addition, when two or more samples or subjects in a group are measured, there is generally variation due to individual differences. For example, the weight of each volunteer in the in vivo study performed for this research was widely different.

For practical purposes, a few measurements of a given sample are usually performed, and the result averaged.

Common standard statistical calculations in pharmacokinetic studies include (Shargel, Wu-Pong et al. 2004):

1) The mean or average, which represents the sum of the observations divided by the number, $n$, of observations, and is a frequently used term in statistics to generalize the nature of the data and provide a measure of central tendency.

2) The mean \pm standard deviation (SD). The standard deviation gives an indication of the spread of data points around the mean. A standard deviation relative to the mean value is indicative of good consistency and reproducibility of the measurements. A large standard deviation indicates poor consistency and data fluctuations. 
3) The relative standard deviation (RSD), or coefficient of variation (CV) expressed as a percentage, represents the ratio of the standard deviation to the mean. It is a way of expressing variability on a percent basis and is useful for comparing the degree of variation from one set of measurements or calculations to another when the means are different.

4) Other descriptive terms used to give a measure of central tendency are the median, which is the middle value of the observations between the highest and lowest value, and the mode, which represents the most frequently occurring value.

5) The term range is used to describe the dispersion of the observations and is the difference between the highest and lowest values. For data that are distributed as a normal (or Gaussian) distribution (i.e., the bell-shaped curve which describes the distribution of the frequency of the measurements drawn randomly from a population when data tends to be around a central value) the mean, median, and mode have the same value.

6) Analysis of Variance (ANOVA) is a method used to test differences between two or more means. An ANOVA on two groups is similar to the $t$-test, which tests whether the means of two groups are statistically different from one another. ANOVA methods can estimate the variance among different subjects (intersubject variability), groups, or treatments. 


\section{$1.7 \quad$ In vitro-in vivo correlations}

\subsubsection{Importance of in vitro-in vivo in the development and optimization of dosage forms}

A key goal in the development of pharmaceutical dosage forms is a good prediction of their in vivo performance. Until the early 1960s, disintegration tests were the only official in vitro tests used by major pharmacopoeias all over the world as a means of predicting in vivo drug-release and behaviour. However, because of the critical importance of drug dissolution and release from a dosage form under physiological conditions, especially for drugs that are highly absorbed or for SR dosage forms, in vitro dissolution is more relevant than disintegration as a way to describe what will possibly happen in vivo. In the 1970s, a series of studies showed that the mean dissolution time resulting from IR tablets could be related to pharmacokinetic parameters such as the rate and extent of drug absorption, thus establishing a correlation between in vivo and in vitro performance (Johnson, Greer et al. 1973; Lindenbaum, Butler Jr et al. 1973; Shaw, Raymond et al. 1973). These results supported the incorporation of dissolution tests and specifications into the United States Pharmacopeia (USP) General Chapters and monographs on solid oral dosage forms, which substituted disintegration tests as the official methodology (Jorgensen and Bhagwat 1998). In the following years, dissolution equipment was improved and standardized, and calibration tests were instituted. This led to the inclusion of dissolution tests in all monographs for solid oral dosage form and their use as a good means of ensuring the uniformity of IR dosage forms (Cohen, Hubert et al. 1990). In the early 1980s, the USP addressed the dissolution of extended-release dosage forms ('sustained-release' herein). Since then, with the advancement of technology, progress in drug delivery research and a crescent emphasis on the predictability of in vivo behaviour of dosage forms by means of in vitro tests, dissolution tests have gained more popularity (Zahirul and Khan 1996). It was, therefore, necessary to further develop a technique to reliably correlate in vitro and in vivo drug-release data, known as in vitro-in vivo correlations (IVIVC).

The term correlation is employed in this context to express the relationship between appropriate in vitro release characteristics and in vivo bioavailability parameters. Various definitions of IVIVC have been proposed. The FDA has defined an IVIVC as "a predictive 
mathematical model describing the relationship between an in vitro property of a dosage form and a relevant in vivo response". Usually, the in vitro property is the rate or extent of drug dissolution or release while the in vivo response is the plasma drug concentration or amount of drug absorbed (Guidance for Industry. Dissolution Testing of Immediate Release Solid Oral Dosage Forms. FDA. (1997)). The United States Pharmacopoeia (USP) Subcommittee on Biopharmaceutics defines IVIVC as "the establishment of a rational relationship between a biological property, or a parameter derived from a biological property produced by a dosage form, and a physicochemical property or characteristic of the same dosage form" (US Pharmacopeial Convention, 2013). Typically the parameter derived from the biological property is the $\mathrm{AUC}_{0-\infty}$ or $C_{\max }$, while the physicochemical property is the in vitro dissolution profile.

IVIVC has different applications in the pharmaceutical industry, regulatory agencies and academia. The main objective of IVIVC is to serve as a surrogate for in vivo bioavailability data. Thus, it is used to support the request of waivers of in vivo bioavailability and bioequivalence studies (biowaivers) from regulatory authorities. Besides, IVIVC can be used in the early stages of the development of new dosage forms to reduce the number of studies in human volunteers during formulation development. Likewise, IVIVC are applied in certain scale-up and post-approval changes (SUPAC), for example, to optimize formulations or to change manufacturing processes or equipment, as these changes may also require human studies to prove that the new formulation is bioequivalent with the old one. Therefore, IVIVC can significantly reduce the time and cost associated with both development and optimization of formulations by avoiding lengthy and expensive clinical trials. Also, IVIVC can be employed to establish meaningful dissolution specifications and to support and/or validate the use of dissolution methods specifications. A validated in vitro test that is discriminative and correlated with the in vivo performance is usually used in quality control to assure that each batch of the same product will perform identically in vivo (Guidance for industry. Extended release oral dosage forms: development, evaluation and application of in vitro/in vivo correlations. FDA. (1997)).

The BCS has been referenced as a guideline for determining the conditions under which IVIVC are expected on the basis of the solubility and gastric permeability of drugs. According to the BCS, IVIVC is generally anticipated for IR products containing Class II 
drugs (low solubility and high permeability). It may also be possible for Class I drugs (high solubility and high permeability). However, in the case of Class I drugs, a correlation is only possible if the dissolution rate is slower than the gastric emptying rate (Amidon, Lennernas et al. 1995).

Compared to IR dosage forms, an IVIVC is, in general, more easily established for SR dosage forms where dissolution and not absorption is the rate-limiting phenomena in the absorption process (Demirturk and Öner 2003). Depending on the design of the SR product, the apparent absorption takes place in the small intestine, ascending colon and/or throughout the large intestine (Shameem, Katori et al. 1995). A longer absorption phase over a prolonged period of time makes it easier to develop Level A, B, C or Multiple Level C IVIVC models. However, the feasibility of an IVIVC for a SR dosage form depends on drug properties, delivery technology, and formulation design (Qiu 2009).

Because of the importance of IVIVC in the development of dosage forms, the ability to predict in vivo dissolution/absorption characteristics from dissolution data of SR dosage forms has become one of the most important aspects of developing SR products. The use of in vitro dissolution to evaluate and predict in vivo performance of SR drug products through IVIVC was supported by the publication of a guidance on IVIVC by the FDA in 1997 (referred to as 'modified release' in this document) (Guidance for industry. Extended release oral dosage forms: development, evaluation and application of in vitro/in vivo correlations. FDA. (1997)).

Regulatory flexibility for certain formulation and process changes regarding SR products is allowed when a direct correlation between the in vitro drug-release and in vivo absorption exists (Guidance for Industry SUPAC-MR: Modified Release Solid Oral Dosage Forms. FDA. (1997)).

Another link between in vitro drug product performance and in vivo drug product biopharmaceutical and pharmacokinetic performance reported in the literature is the in vitro-in vivo relationship (IVIVR). IVIVR is a more general term, which comprises the broad range of activities involved in relating in vitro dissolution to in vivo absorption and non-linear relationships. The most simple and appropriate relationships to consider first is the linear relationship, nonetheless non-linear correlations, even if uncommon, might be appropriate in some cases (Emami 2006). 
Generally, when the dissolution profile is not affected by factors such as $\mathrm{pH}$, surfactant, ionic strength, enzyme, osmotic pressure, agitation intensity, a set of dissolution data obtained from one formulation is correlated with the corresponding in vivo response in order to develop a correlation. When the dissolution rate depends on those factors, the dissolution profiles from formulations with different in vitro release rates as, for instance, slow, medium and fast (Figure 1.28 (A)), should be determined using a discriminating in vitro test methodology. The in vitro release rates, as measured by cumulative percentage of drug dissolved for each formulation studied, should differ adequately (e.g., by 10\%) (Guidance for industry. Extended release oral dosage forms: development, evaluation and application of in vitro/in vivo correlations. FDA. (1997)). The corresponding in vivo response can be plasma concentrations or, more frequently, the amount of drug released and/or absorbed in vivo. The latter is obtained from the observed plasma concentration-time curve by deconvolution. The resulting in vivo profiles should show a comparable difference, i.e., a $10 \%$ difference in the pharmacokinetic parameters of interest between each formulation (Guidance for industry. Extended release oral dosage forms: development, evaluation and application of in vitro/in vivo correlations. FDA. (1997)). These data may derive from studies at early or late stages of dosage form development, such as bioavailability studies conducted in the formulation screening stage or an in vivo study specifically designed to explore IVIVC (Mojaverian, Rosen et al. 1997; Dutta, Qiu et al. 2005).

\subsubsection{Correlation levels}

The publication in 1997 of the FDA's Guidance for Industry: Extended Release Oral Dosage Forms: Development and Application of In Vitro/In Vivo Correlations formalized the classification of the IVIVC levels into Levels A, B, C, and Multiple C, depending upon the type of data used to establish the relationship and the ability of the correlation to predict the entire plasma profile of a dosage form.

Level A correlation (Figure 1.28) is a point-to-point relationship between the in vitro release time course and the in vivo response time course (e.g., the time course of plasma drug concentration or amount of drug absorbed) and represents the highest category of correlation 
(US Pharmacopeial Convention, 2013). To develop such a correlation all of the dissolution and plasma concentration data available are used, and the mathematical description for the in vitro dissolution and in vivo input rate is the same. This direct relationship permits the determination of the in vivo drug-release rate of the dosage form using the measurement of in vitro dissolution rate alone. Therefore, an in vitro dissolution curve can serve as a surrogate for in vivo performance (Emami 2006).

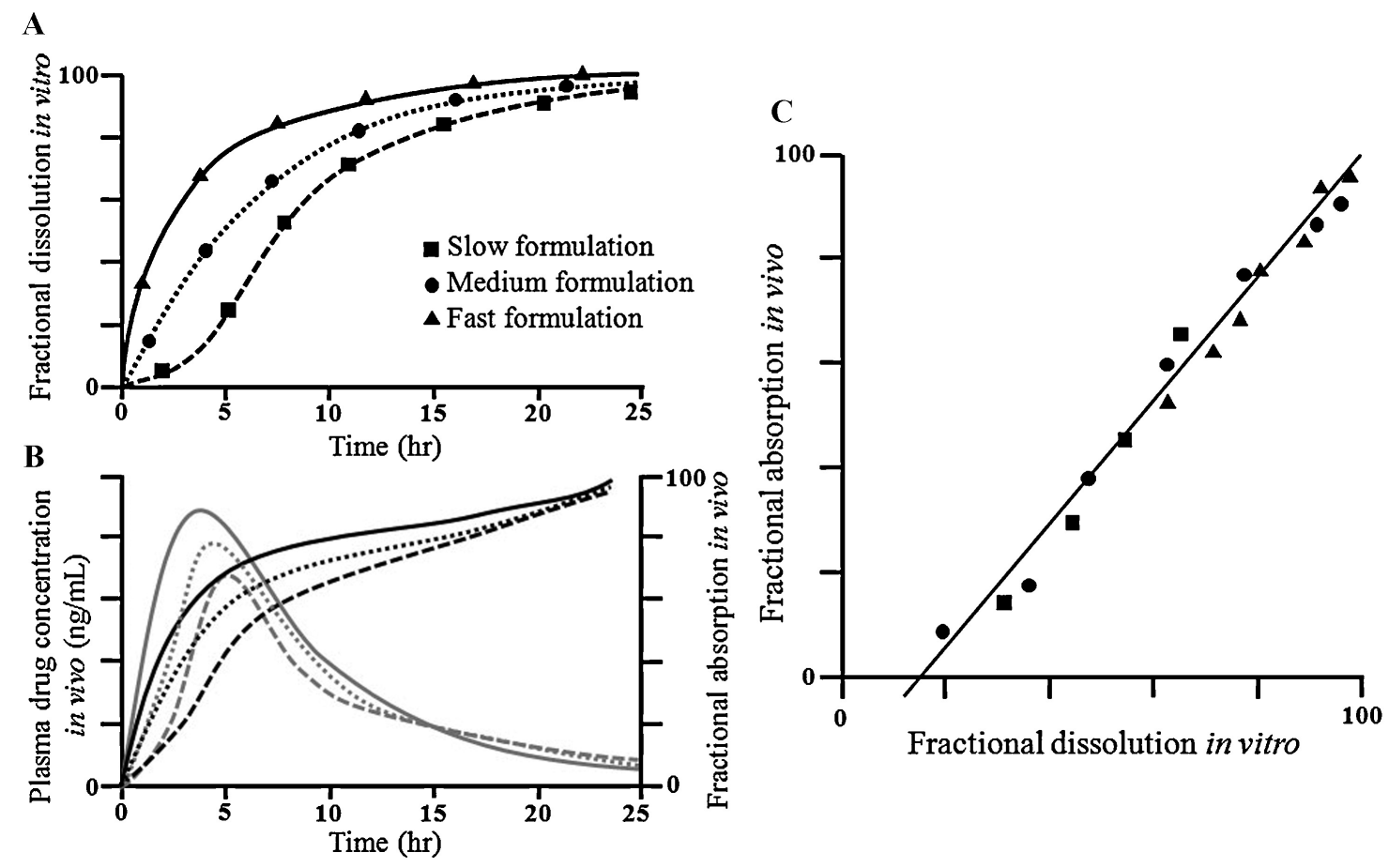

Figure 1.28 Example of Level A IVIVC. (A) In vitro dissolution profiles of slow ( $\mathbf{\square}:$ ),

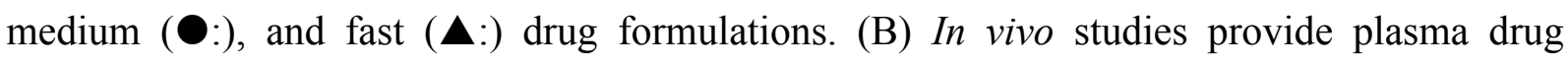
concentration of each formulation (gray lines), which can be converted to fractional absorption profile (black lines) by deconvolution. (C) Level A IVIVC can be derived from the fractional dissolution in vitro and the fractional absorption in vivo. Figure reproduced from ( $\mathrm{Lu}, \mathrm{Kim}$ et al. 2011) with permission of Elsevier Ltd. 
A Level A correlation is typically attempted for SR dosage forms (Long and Chen 2009). A validated Level A IVIVC can be used to obtain waivers of bioequivalence studies, set dissolution specifications, and form the basis for justifying scale-up and post-approval manufacturing changes (Long and Chen 2009). The percentage of drug absorbed may be calculated by means of model dependent techniques such as Wagner-Nelson procedure or Loo-Riegelman method or by model-independent numerical deconvolution.
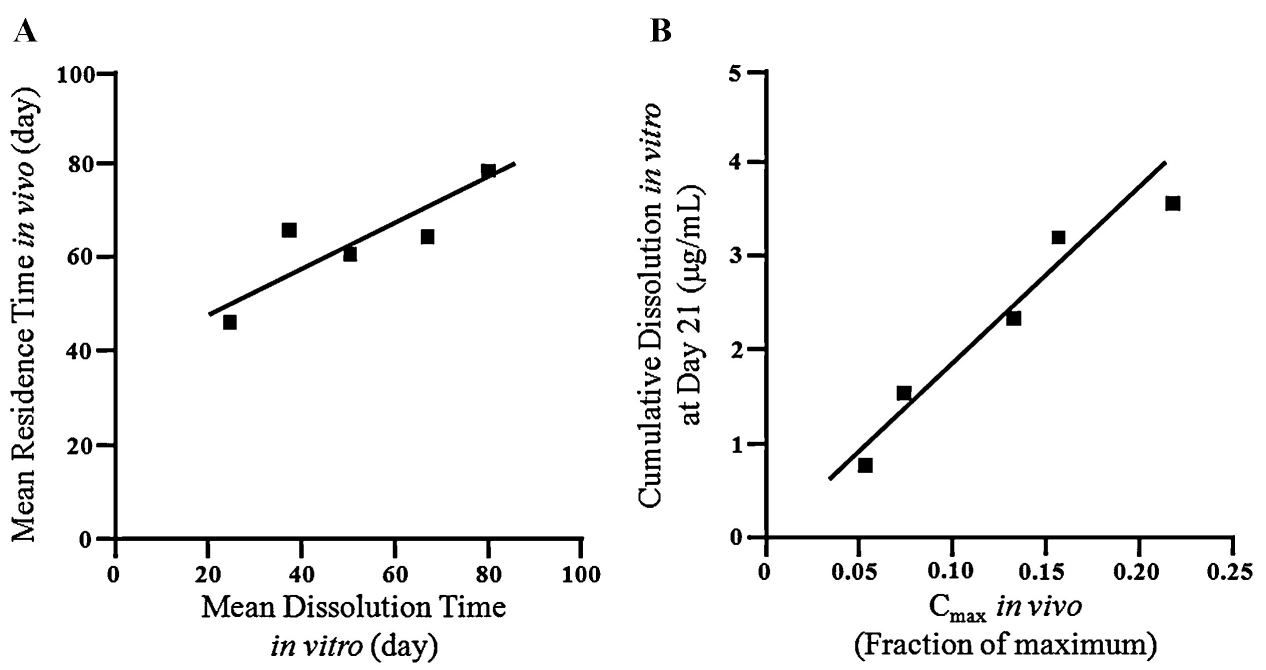

Figure 1.29 Example of Level B (A) and Level C (B) IVIVC. Figure reproduced from (Lu, Kim et al. 2011) with permission of Elsevier Ltd.

Level B correlation (Figure 1.29 (A)) is a relationship between the mean in vitro dissolution time $\left(M D T_{\text {vitro }}\right)$ of the product and the mean in vivo dissolution time $\left(M D T_{\text {vivo }}\right)$ or the mean in vivo residence time (MRT), each obtained by application of statistical moment analysis to the full release profile. Although a Level B correlation uses all of the in vitro and in vivo data it is not considered to be a point-to-point correlation because it does not reflect the actual in vivo plasma concentration curve and a number of different in vivo curves can generate similar $M R T$ values (Guidance for industry. Extended release oral dosage forms: development, evaluation and application of in vitro/in vivo correlations. FDA. (1997)). Therefore, it is not possible to rely only on a Level B correlation to justify modifications, such 
as formulation and excipient source. In addition, in vitro data from such a correlation could not be used to justify the extremes of quality control standards (US Pharmacopeial Convention, 2013).

Level C correlation (Figure 1.29 (B)) is a level for which a particular element of the dissolution profile (e.g., the cumulative dissolution in vitro at day 21, or the time required for dissolution of a certain amount, such as $t_{50 \%}$ ) is related to a single mean pharmacokinetic parameter (e.g., AUC, $T_{\max }$ or $C_{\max }$ ). Therefore, it does not reflect the entire shape of the plasma drug concentration curve, which is an essential factor to evaluate the performance of SR products. This is the weakest level of correlation as only partial relationship between absorption and dissolution is established. Consequently, the usefulness of a Level C correlation in predicting in vivo drug performance as well as formulation and manufacturing changes is limited. Biowaiver is generally not possible using this correlation level (Guidance for industry. Extended release oral dosage forms: development, evaluation and application of in vitro/in vivo correlations. FDA. (1997)). Besides, just as level B, this level of correlation is not useful in justifying the extremes of quality control standards (US Pharmacopeial Convention, 2013). However, Level C correlation can be useful in early stages of formulation development when pilot formulations are being selected (Qiu 2009). It can also be used for IR products, where the time course for absorption is of short duration, and obtaining a sufficient number of data points in the in vivo profile for a Level A IVIVC is more difficult (Long and Chen 2009).

The utility of a Level $\mathrm{C}$ correlation can be strengthened by using information from multiple time points, and creating a Multiple C correlation (Long and Chen 2009). This type of correlation relates the amount of drug dissolved at several time points of the dissolution profile to one or several pharmacokinetic parameters of interest $\left(C_{\max }\right.$, AUC, or any other suitable parameters). As opposed to level B and level C, a multiple point Level C correlation may be used to justify a biowaiver, provided that the correlation has been established over the entire dissolution profile with one or more pharmacokinetic parameters of interest (Emami 2006). A multiple $\mathrm{C}$ correlation should be based on at least three dissolution time points covering the early, middle, and late stages of the dissolution profile. If such a multiple Level C correlation is achievable, then the development of a Level A correlation may also be possible (Emami 2006). 


\subsubsection{Considerations in dissolution test method development for IVIVC}

\subsubsection{Importance of dissolution testing}

Dissolution testing is essential in all stages of drug product development. In the early stages of formulation design and optimization, in vitro dissolutions are performed to support the choices made between different formulation candidates. These choices are based on critical information on possible risks such as dose dumping, food effects, the interaction of drug substances with other formulation components, and other important parameters for formulation performance. At these stages, dissolution testing also helps to understand the drug-release mechanism. Evaluation and analysis of drug-release should not only include quantitative evaluations using HPLC or spectrophotometry but also visual observations of the performance of dosage forms. Mathematical models, such as the Peppas et al. model, can be used to indentify formulations design variables and dissolution method parameters which can significantly influence drug-release (Long and Chen 2009). The development process is then characterized by further cycles of formulation optimization and performance testing, both in vivo and in vitro, during which IVIVC are established. These cycles culminate in the determination of the final formulation. Since there is no other in vitro performance test with such a close link to in vivo performance, dissolution and drug-release studies are a regulatory requirement for the development, and final approval of all solid oral dosage forms (US Pharmacopeial Convention, 2013). After product approval, dissolution testing is used to assess

product quality and to evaluate formulation and manufacturing changes (Guidance for Industry SUPAC-MR: Modified Release Solid Oral Dosage Forms. FDA. (1997)).

Although dissolution tests have been successfully implemented on IR dosage forms by regulatory authorities, there have been great difficulties in standardizing dissolution test conditions and parameters for testing SR oral dosage forms. These difficulties are mainly due to the fact that these dosage forms are usually designed for prolonged residence time in the gastrointestinal tract and, therefore, the drug-release profile will be more susceptible to the variabilities in the in vivo conditions (e.g., presence and nature of food in the gastrointestinal tract or time of the day the dosage form is administered), which are, in turn, very difficult to simulate. However, the creative use of dissolution techniques can speed the early stages of 
formulation development, particularly in the case of SR products. Therefore, even dissolution methods which fail to meet compendial requirements may still provide advantages in product development (Jorgensen and Bhagwat 1998).

Through the use of dissolution tests it is possible to obtain a measure of the extent and rate of drug-release from a dosage form into an aqueous medium under a set of specific test conditions. The drug-release profile is the result of a combination of factors, in particular, the physicochemical properties of the drug, the formulation design and manufacturing process, and the chemical and mechanical conditions of the test method selected to investigate drugrelease. Therefore, the development of a dissolution test method must include an evaluation of the contribution and influence of these factors to drug-release (Long and Chen 2009). In the particular case of a new SR dosage form, the development of a test method should also involve an assessment of the physiological conditions the dosage form will encounter throughout its total residence time in the body, so that the release profile of the drug is tested in an environment closely related to the actual in vivo conditions. This assessment is particularly important for dosage forms that will have different release/absorption profiles at distinct physiological conditions of the gastrointestinal tract. The more information that is available, the easier it is to design an initial set of dissolution test conditions (Bowker 1996; Zahirul and Khan 1996; Zahirul and Khan 1996). However, while it is possible to have, nowadays, a certain control of the drug-release profiles of a dosage form in vitro, it is not always possible to obtain the desired drug-release and therapeutic plasma concentrations in vivo.

The key considerations in dissolution test method development are the apparatus type employed, along with its associated operating parameters, and the medium composition. A brief description of these aspects will follow.

\subsubsection{Dissolution apparatus selection}

The type of dosage form under investigation is the primary consideration in apparatus selection. The USP Chapter $<711>$ describes four types of dissolution apparatus used in dissolution studies (US Pharmacopeial Convention, 2013) and has been harmonized with the 
European and the Japanese Pharmacopoeias. These apparatuses are also recommended in the FDA Guidance for Industry, Dissolution Testing of Immediate Release Solid Oral Dosage Forms (Guidance for Industry. Dissolution Testing of Immediate Release Solid Oral Dosage Forms. FDA. (1997)). Guidance for the development and application of dissolution testing is also provided by the International Pharmaceutical Federation (FIP) (International Pharmaceutical Federation. FIP guidelines for dissolution testing of solid oral products, Final draft (1996)). These apparatuses will not be discussed in detail in this thesis. For detailed descriptions of the apparatuses, the reader is referred to a book by Dressman J. (Dressman 2005). Apparatus 1 (rotating basket) and Apparatus 2 (paddle assembly) (Figure 1.30) are the most widely used for dissolution testing of solid dosage forms (IR as well as SR products), mostly because they are simple, robust, and adequately standardized apparatus designs, and are supported by a wider experience of experimental use than the other types of apparatuses (Dressman 2005). Apparatus 3 (reciprocating cylinder) and 4 (flow-through cell) were added to the USP in 1990 for the convenience of European companies that were using them to evaluate MR products. Three other apparatus have been described. Apparatus 5 (paddle over disk), 6 (rotating cylinder) and 7 (reciprocating holder) have been used mainly for transdermal dosage forms. Apparatus 7 has also been used for oral MR dosage forms using osmotic pump technology (Long and Chen 2009). 


\section{USP Apparatus 1}

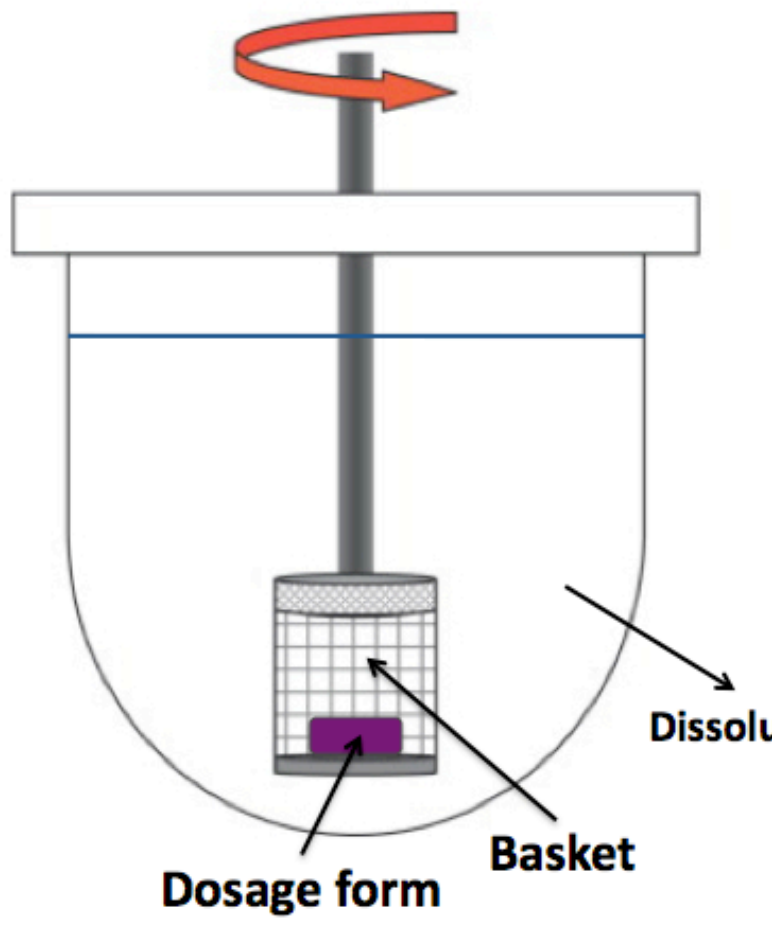

USP Apparatus 2

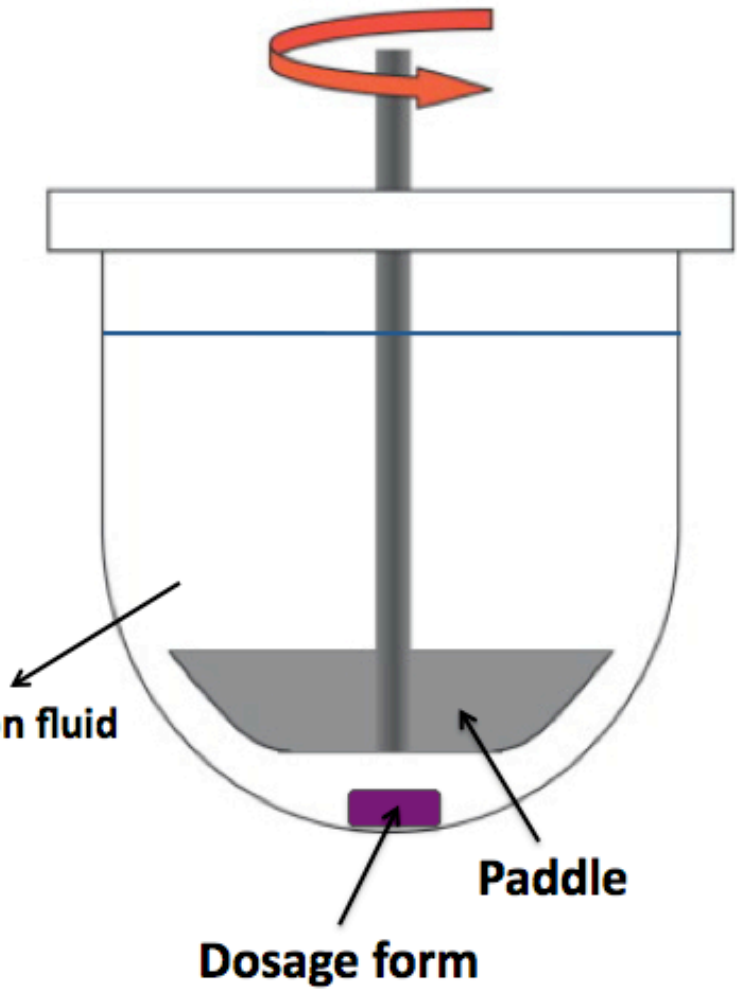

Figure 1.30 Schematic representation of Apparatus 1 (rotating basket) and Apparatus 2 (paddle assembly) dissolution vessels.

Both Apparatuses 1 and 2 use single hemispherical bottom vessels with a centred spindle. The vessels contain the dissolution medium, typically 500-1000 mL with $900 \mathrm{~mL}$ most frequently used. The vessels are fitted with covers containing sampling ports and immersed in a water bath kept at a constant temperature as a means of controlling the temperature of the media in the vessels. The major difference between the two Apparatuses relate to how the dosage form is placed inside the vessel. For Apparatus 1, the dosage form is placed in a basket attached to the end of the spindle, while for Apparatus 2 the dosage form is dropped directly into the vessel. Apparatus 1 induces agitation by rotating the basket and apparatus 2 by using a rotating paddle. When the dosage form floats (e.g., capsules), Apparatus 1 can be used. Alternatively, sinkers designed to enclose the dosage form can be used with Apparatus 2. However, for hydrophilic matrix systems that swell during dissolution, 
USP Apparatus 1 may not be appropriate because the matrix can obstruct the holes in the basket, disrupting the hydrodynamics and slowing dissolution. In addition, the basket may entrap the swelling matrix and damage its structure. The same trouble was demonstrated for dosage forms placed inside sinkers used in apparatus 2 (Soltero, Hoover et al. 1989).

Sampling from Apparatuses 1 and 2 can be accomplished either manually or automatically, with filtration to eliminate undissolved particles.

For any selected apparatus, concentration of each sample is measured by a suitable analytical method. The concentration of each sample is then converted to the amount of drug released using an algorithm, taking into account the sampling method and whether replacement of media occurred. The most common test methods use either UV/visible spectrophotometry or HPLC with UV detection (Long and Chen 2009). Measuring absorbance at UV or visible wavelengths requires minimal to no sample preparation, which is an advantage. However, when using spectrophotometry there is the possibility of assay interference by excipients that absorb in the same wavelength region or by any undissolved excipients that may scatter light and affect the results. These interferences can be eliminated by using a baseline normalization approach or by using second derivatives of the absorbance

profile (Perkampus 1992). This interference is less problematic when using HPLC because of its improved specificity resulting from the chromatographic separation. However, HPLC is a technique that requires large amounts of organic solvents, and thus it is more expensive and bad for the environment.

\subsubsection{Dissolution test media and methods}

Evaluation of the appropriate testing media begins by considering the in vivo transit of an oral dosage form. However, there is no in vitro test that can physically reproduce the in vivo release environment given the complex nature of the gastrointestinal tract, the factors that affect its activity and the diverse mechanisms employed to achieve sustained drug-release (Qiu, Garren et al. 2003). Nevertheless, it is possible to develop a predictive dissolution test when in vivo drug-release is the chief controlling factor in the rate of appearance of the drug in the blood (Qiu 2009), which is the case with SR dosage forms. 
Media and test conditions for SR products should be able to tackle at least some of the variables encountered in vivo. They should try to simulate 1) the $\mathrm{pH}$ range throughout the gastrointestinal tract; 2) the approximate time the SR dosage form remains at each $\mathrm{pH}$ in the digestive system; 3) food induced physiological changes, or at least in part, that occur in the gastrointestinal tract; and 4) the motility pattern and other mechanical forces encountered by the dosage form in the gastrointestinal tract. Therefore, an IVIVC for a SR product should be developed on a case-by-case basis (Qiu 2009). Contrariwise, for IR dosage forms, a lower $\mathrm{pH}$ medium simulating the conditions in the stomach is more appropriate because these products are designed to release drug rapidly, usually in less than an hour.

Water is not the most preferred medium because water has no buffering capacity. The use of buffered media is even more important when the dissolution of either the drug or excipients is $\mathrm{pH}$-sensitive. In unbuffered media, dissolution of ionizable species may create a $\mathrm{pH}$-microclimate where the $\mathrm{pH}$ at the surface of the particles or dosage form is different from the bulk solution $\mathrm{pH}$. In addition, the dissolution of an ionizable drug from a dosage form containing a high drug loading may create changes of the $\mathrm{pH}$ of the bulk medium throughout the duration of the test. In either case, these changes in $\mathrm{pH}$ add an uncontrolled variable to the events taking place during dissolution, and may complicate the interpretation of results (Long and Chen 2009). It has also been demonstrated that an improvement of the correlation can be attained when the in vitro test is done in a $\mathrm{pH}$ gradient instead of distilled water (Ranade 1991). The typical $\mathrm{pH}$ range for dissolution testing is from $\mathrm{pH} 1$ (representing gastric $\mathrm{pH}$ ) to pH 7.5 (representing intestinal pH) (Long and Chen 2009).

There have been many attempts to simulate food affects in vitro - for example, surfactants have been added to the dissolution media (Qiu, Cheskin et al. 1997; Dressman, Amidon et al. 1998; Galia, Nicolaides et al. 1998), dosage forms have been pre-soaked in peanut oil or placed in continuous oil contact to mimic the presence of a high fat meal $(\mathrm{Mu}$, Tobyn et al. 2003), or tested in different dissolution apparatuses under varied hydrodynamic, mixing conditions to examine the effects of mechanical destructive forces on drug-release from MR dosage forms (Shameem, Katori et al. 1995). These methods are, however, only capable of imitating one or two aspects of physiological conditions. A very complicated in vitro model has been described, which consists of a multi-compartment dynamic computer- 
controlled system designed to simulate the human stomach and small intestine (TIM-1), and large intestine (TIM-2), respectively, and intended for studying the behaviour of oral drug dosage forms under various physiological gastrointestinal conditions (Minekus, SmeetsPeeters et al. 1999; Blanquet, Zeijdner et al. 2004).

Surfactants in dissolution media have been used not only during early formulation development to simulate the intestinal state or for correlation with in vivo studies but also to increase drug solubility of poorly soluble drugs, because the solubility in buffer solutions may be insufficient to allow for the use of a practical volume of medium (Shah, Konecny et al. 1989; Abrahamsson, Johansson et al. 1994). This approach is not far from real physiological conditions, since bile salts and lecithin are natural surfactants present in intestinal fluids and bile, and are responsible for solubilizing poorly soluble nutrients before absorption (Hörter and Dressman 2001).

Sink conditions during a dissolution experiment are usually required to obtain in vitro dissolution curves representing the biopharmaceutical properties of the drug product under investigation with minimal effects due to the influence of solubility (Emami 2006). The term 'sink conditions' denotes a state in which the concentration of the drug in a solubilizing medium is very low compared with that of a saturated solution of the drug in the same medium (Pillay and Fassihi 1998). According to the USP, sink conditions are achieved when the saturated solubility of the drug in the dissolution medium is at least three times the concentration of a completely dissolved tablet in the volume of media used in the test. Thus, sink conditions are achieved depending on the solubility of the drug in the medium, the drug dose, and the selected volume.

Since the human body temperature is about $37^{\circ} \mathrm{C}$, standard dissolution testing is carried out at this temperature with an acceptable variation of $\pm 0.5^{\circ} \mathrm{C}$ in most pharmacopoeias.

Another important consideration is drug stability during the dissolution test. A typical dissolution test at $37^{\circ} \mathrm{C}$ for SR dosage forms runs $12-24 \mathrm{~h}$ or more, and quantification of the samples can take additional time. Therefore, the stability of the drug in the medium at $37^{\circ} \mathrm{C}$ and as a function of $\mathrm{pH}$ should be evaluated (Jorgensen and Bhagwat 1998). Susceptibility to light or oxidation should also be evaluated for additional precautions. 
When choosing the dissolution test conditions that are appropriate for a certain SR dosage form, it should also be taken into consideration that the release characteristics of many SR products show some $\mathrm{pH}$ dependence and may be affected by the physicochemical properties of the drug and by the excipients in the formulation to varying degrees depending upon the dissolution test conditions. For example, the swelling of polymers used as ratecontrolling agents can be affected by medium $\mathrm{pH}$, ionic strength, surfactants, and even counterion composition (Long and Chen 2009).

Although it is important that in vivo hydrodynamic conditions are simulated during dissolution testing, the variability in motility patterns of the gastrointestinal tract in fasted and fed objects complicates the task of setting a unique agitation condition during in vitro testing (Zahirul and Khan 1996). However, in the case of SR products, the release is typically controlled by polymers. Since polymer behaviour, such as swelling or entanglement, may be sensitive to $\mathrm{pH}$ or ionic strength, the medium composition will usually play a greater role than the hydrodynamics in influencing drug-release (Long and Chen 2009).

In conclusion, a dissolution methodology that is able to discriminate between the study formulations with different release patterns and best reflects the in vivo behaviour should be used to establish an IVIVC (Emami 2006). It is generally easier to obtain a good IVIVC with SR dosage forms that are essentially unaffected by variables such as $\mathrm{pH}$, agitation or ionic strength (Guidance for Industry SUPAC-MR: Modified Release Solid Oral Dosage Forms. FDA. (1997)).

In the development of a Level A IVIVC, the in vitro test conditions, such as the agitation rate, choice of apparatus and $\mathrm{pH}$ and temperature of the medium, may be modified in order to alter the dissolution profile and facilitate the establishment of a correlation. The shape of the in vivo profile can then be used as a target for the in vitro data to match. Statistics and the tools for profile comparisons, which will me discussed in the next section, facilitate efficient development (Qiu 2009). However, once a discriminating system is developed, dissolution conditions should be the same for all formulations tested in the study and should remain unchanged before further steps towards correlation evaluation are undertaken (Guidance for industry. Extended release oral dosage forms: development, evaluation and application of in vitro/in vivo correlations. FDA. (1997)). 


\subsubsection{Statistical comparison of dissolution profiles}

Model-dependent and model-independent methods can be used to quantitatively compare dissolution profiles in order to evaluate sameness (Sathe, Tsong et al. 1996; Pillay and Fassihi 1998; Yuksel, Kanık et al. 2000; Costa and Sousa Lobo 2001). The modeldependent methods rely on selecting the proper mathematical model and comparing the goodness of fit and changes in fitted parameters. The mathematical models are fitted to individual dissolution data using non-linear regression, resulting in the estimation of the model parameters with their standard errors and descriptive statistics of regression for each model. These estimations allow the determination of the suitable mathematical models to describe the dissolution profiles. Common statistical tools, such as $t$-tests or ANOVA, can be used to demonstrate sameness of the fitted parameters (Long and Chen 2009). Model-independent approaches also use the dissolution data in their native form. These methods provide a direct comparison of a test profile against a reference profile without any transformation to another mathematical expression. Calculations that can be made include the area under the curve of the percent released versus time profile or the amount released at a particular time point or time points (Tsong, Hammerstrom et al. 1996; Yuksel, Kanık et al. 2000). Although there measurements may demonstrate the equivalence of the amount of drug released, they will not necessarily indicate whether the shape of the profile is similar (Long and Chen 2009).

The most commonly used model-independent approach is the fit factors, which were introduced by Moore and Flanner (Moore and Flanner 1996) and consist of the difference factor, $f_{1}$, and the similarity factor, $f_{2}$. The $f_{2}$ is more frequently reported than the $f_{1}$. The $f_{2}$ is a logarithmic transformation of the average sum squared error and is given by the following equation:

$$
f_{2}=50 \times \log \left\{\left[1+\left(\frac{1}{n}\right) \sum_{t=1}^{n}\left(R_{t}-T_{t}\right)^{2}\right]^{-0.5} \times 100\right\}
$$

Equation 19

where $R_{t}$ and $T_{t}$ are the mean percent dissolved of the reference and test products at each time point $t$, and $n$ is the number of time points in the profile. 
Two dissolution profiles are similar if $f_{2}$ is between 50 and 100, according to the FDA and the European Agency for the Evaluation of Medical Products (EMEA). However, the closer to $100(\%) f_{2}$, the more similar the dissolution profiles are. If $f_{2}$ is 100 , the two profiles are identical. An $f_{2}$ of 50 indicates there is a $10 \%$ difference between profiles (Shah, Tsong et al. 1998).

The $f_{l}$ provides the mean percent difference between two curves and is calculated as:

$$
f_{1}=\left\{\left[\sum_{t=1}^{n}\left(R_{t}-T_{t}\right)\right] / \sum_{t=1}^{n} R_{t}\right\} \times 100
$$

Identical profiles will have an $f_{l}$ equal to zero and a value less that $15 \%$ is commonly taken to indicate sameness of profiles (Long and Chen 2009).

ANOVA-based methods have also been applied to compare dissolution profiles. These methods use the dissolution data in their native form or as simple transform and the analysis is capable of showing differences between profiles in level and shape. The latter characteristic is particularly important to identify differences in the dissolution mechanism (Yuksel, Kanık et al. 2000).

\subsubsection{Bioavailability studies for development of IVIVC}

Bioavailability is a measure of systemic availability of a drug and reflects the rate and extent at which the drug is absorbed from a drug product and becomes available to the site of action (Shargel, Wu-Pong et al. 2004). In the development of an IVIVC, a bioavailability study should be performed to characterize the plasma concentration versus time profile for each formulation (Uppoor 2001).

A bioavailability study protocol in humans consists of several elements. For example, the study design should include test and reference products, dosage regimen, blood sampling times, fasting/meals schedule and analytical methods. In addition, the information on volunteer selection includes inclusion/exclusion criteria and medical history as well as physical examination. For example, the study might exclude any volunteers who have known 
allergies to the drug, are overweight, or have taken any medication within a specific period (often one week) prior to the study. It is also important to refer in the protocol some ethical considerations, such as informed consent, side effect risks and physiological effects, and emergency procedures. A section relative to data analysis should also be part of the protocol, among others (Shargel, Wu-Pong et al. 2004).

The number of subjects in a typical bioavailability study will depend on the expected inter-subject and intra-subject variability (Shargel, Wu-Pong et al. 2004). However, according to the FDA guidance (Guidance for industry. Extended release oral dosage forms: development, evaluation and application of in vitro/in vivo correlations. FDA. (1997)), bioavailability studies for IVIVC development should be performed in humans with enough subjects to adequately characterize the absorption profiles of the drug products.

Both the parent drug and its major metabolites are generally measured using a selective, accurate and precise analytical method. Studies on the influence of food are generally carried out using meal conditions that are expected to provide the greatest effects on gastrointestinal physiology in order to induce the utmost effects on systemic drug bioavailability (Shargel, Wu-Pong et al. 2004).

Crossover studies, in which each volunteer receives all treatments that are being investigated in a randomized order and at different times, are preferred to establish a IVIVC, since they reduce inter-subject variability. However, parallel studies or cross-study analyses may also be acceptable. The reference product can be an intravenous solution, an aqueous oral solution or an IR product of the drug. In addition, IVIVC are usually developed in the fasted state. When a drug is not tolerated in the fasted state, studies may be conducted in the fed state (Guidance for industry. Extended release oral dosage forms: development, evaluation and application of in vitro/in vivo correlations. FDA. (1997)).

The in vivo release/absorption profile from plasma concentration-time data is obtained using an appropriate deconvolution technique. Subsequently, in the development of a Level A IVIVC, the calculated percentage absorbed or released in vivo is correlated with the percentage released in vitro using a basic linear model with intercept (a) and slope (b):

$$
(\% \text { absorbed })_{\text {in vivo }}=\mathrm{a} \times \mathrm{b}(\% \text { released })_{\text {in vitro }}
$$

Equation 21 
Ideally, the relationship should be linear.

The deconvolution technique can be model-dependent or model-independent. Two commonly used model-dependent deconvolution techniques for estimating the apparent in vivo drug absorption profiles following oral administration of a dosage form are Wagner-Nelson (Wagner and Nelson 1963; Wagner and Nelson 1964) and Loo-Riegelman (Loo and Riegelman 1968) methods. These approaches are based on mass balance. The Wagner-Nelson technique is derived from a one-compartment model while the Loo-Riegelman technique is used for a two-compartment model. In 1983, Wagner published an Exact Loo-Riegelman method for absorption analysis of one- to three-compartment models, which contrarily to the Wagner-Nelson method requires intravenous data for the calculation of absorption profiles (Wagner 1983). The model-independent techniques include convolution and deconvolution methods used in linear system analysis (Qiu 2009). For a detailed description of modeldependent and model-independent techniques and their respective equations, the reader is referred to a publication by Emami J. (Emami 2006). In actual applications, software products are frequently used to execute deconvolution and convolution procedures and IVIVC analysis.

\subsubsection{Evaluation of predictability of IVIVC}

Following the establishment of a Level A IVIVC model, it is necessary to demonstrate that predictability of in vivo performance of a drug from its in vitro dissolution characteristics is accurate and consistent, i.e., is maintained over a range of in vitro dissolution release rates and manufacturing changes. The FDA guidance suggests evaluating the goodness of fit by estimating the prediction error (PE), i.e., differences between observed and predicted values over a range of in vitro release rates. Depending on the intended application of the IVIVC and the therapeutic index of the drug, the approaches to validate the model may be internal and/or external. Internal validation is based on the initial in vitro release data used to define the IVIVC model, while external validation requires additional data sets that were not used in the development of the IVIVC (Guidance for industry. Extended release oral dosage forms: development, evaluation and application of in vitro/in vivo correlations. FDA. (1997)). The external validation demonstrates the robustness of the IVIVC. 


\subsection{References}

(Guidance for Industry SUPAC-MR: Modified Release Solid Oral Dosage Forms. FDA. (1997)). Scale-Up and Postapproval Changes: Chemistry, Manufacturing, and Controls; In Vitro Dissolution Testing and In Vivo Bioequivalence Documentation. US Department of Health and Human Services, Food and Drug Administration, Center for Drug Evaluation and Research (CDER).

(Guidance for Industry. Dissolution Testing of Immediate Release Solid Oral Dosage Forms. FDA. (1997)). US Department of Health, Food and Drug Administration, Center for Drug Evaluation and Research (CDER).

(Guidance for industry. Extended release oral dosage forms: development, evaluation and application of in vitro/in vivo correlations. FDA. (1997)). US Department of Health and Human Services, Food and Drug Administration, Center for Drug Evaluation and Research (CDER).

(International Pharmaceutical Federation. FIP guidelines for dissolution testing of solid oral products, Final draft (1996)). Drug Information Journal 30: 1071-1084.

(US Pharmacopeial Convention, 2013). The United States Pharmacopeia 36 - National Formulary 31 . Rockville, MD, USA.

A. Odeku, O. and K. M. Picker-Freyer (2010). "Freeze-dried pregelatinized Dio s c o re a starches as tablet matrix for sustained release." Journal of Excipients and Food Chemicals 1(2): 21-32.

Abrahamsson, B., D. Johansson, et al. (1994). "Evaluation of Solubilizers in the Drug Release Testing of Hydrophilic Matrix Extended-Release Tablets of Felodipine." Pharm Res 11(8): 1093-1097.

Aiache, J. M., S. Aiache, et al. (2001). Initiation à la connaissance du médicament. Paris, Masson.

Alderborn, G. (2002). Tablets and compaction. Pharmaceutics: The Science of Dosage Form Design. M. E. Aulton, Churchill Livingstone: 397-440.

Alderman, D. A. (1984). "A review of cellulose ethers in hydrophilic matrices for oral controlled release dosage forms." Int. J. Pharm. Tech. Prod. Mfr. 5(3): 1-9.

Amidon, G. L., H. Lennernas, et al. (1995). "A theoretical basis for a biopharmaceutic drug classification: the correlation of in vitro drug product dissolution and in vivo bioavailability." Pharm Res 12(3): 413-420.

Artursson, P., K. Palm, et al. (2001). "Caco-2 monolayers in experimental and theoretical predictions of drug transport." Advanced Drug Delivery Reviews 46(1-3): 27-43.

Ashford, M. (2002). Assessment of biopharmaceutical properties. Pharmaceutics: The Science of Dosage Form Design. M. E. Aulton, Churchill Livingstone: 253-274.

Ashford, M. (2002). The gastrointestinal tract - physiology and drug absorption. Pharmaceutics: The Science of Dosage Form Design. M. E. Aulton, Churchill Livingstone: $217-234$.

Ashford, M. (2002). Introduction to biopharmaceutics. Pharmaceutics: The Science of Dosage Form Design. M. E. Aulton, Churchill Livingstone: 213-216.

Atwell, W. A., L. F. Hood, et al. (1988). "The terminology and methodology associated with basic starch phenomena." Cereal Foods World 33(3): 306-112. 
Aulton, M. E. (2002). Dissolution and solubility. Pharmaceutics: The Science of Dosage Form Design. M. E. Aulton, Churchill Livingstone: 15-32.

Baille, W. E., C. Malveau, et al. (2002). "NMR imaging of high-amylose starch tablets. 1. Swelling and water uptake." Biomacromolecules 3(1): 214-218.

Bassi, P. and G. Kaur (2010). "pH modulation: a mechanism to obtain $\mathrm{pH}$-independent drug release." Expert Opin Drug Deliv 7(7): 845-857.

Baumgartner, S., J. Kristl, et al. (2002). "Network structure of cellulose ethers used in pharmaceutical applications during swelling and at equilibrium." Pharm Res 19(8): 1084-1090.

Baumgartner, S., M. Pavli, et al. (2008). "Effect of calcium ions on the gelling and drug release characteristics of xanthan matrix tablets." European Journal of Pharmaceutics and Biopharmaceutics 69(2): 698-707.

Bayne, W., V. Place, et al. (1982 ). "Kinetics of osmotically controlled indomethacin delivery systems after repeated dosing." Clinical Pharmacology \& Therapeutics 32(2): 270-276.

Baynes, R. E. and J. E. Riviere (2010). Chapter 37 - Absorption. Hayes' Handbook of Pesticide Toxicology (Third Edition). K. Robert. New York, Academic Press: 877892.

Benet, L. Z., T. Izumi, et al. (1999). "Intestinal MDR transport proteins and P-450 enzymes as barriers to oral drug delivery." Journal of Controlled Release 62(1-2): 25-31.

Bessems, J. G. and N. P. Vermeulen (2001). "Paracetamol (acetaminophen)-induced toxicity: molecular and biochemical mechanisms, analogues and protective approaches." Crit Rev Toxicol 31(1): 55-138.

Biliaderis, C. G. (1991). "The structure and interactions of starch with food constituents." Canadian Journal of Physiology and Pharmacology 69(1): 60-78.

Billany, M. (2002). Solutions. Pharmaceutics: The Science of Dosage Form Design. M. E. Aulton, Churchill Livingstone: 309-322.

Blanquet, S., E. Zeijdner, et al. (2004). "A dynamic artificial gastrointestinal system for studying the behavior of orally administered drug dosage forms under various physiological conditions." Pharm Res 21(4): 585-591.

Blanshard, J. M. V. (1987). Starch granule structure and function: a physicochemical approach. New York, John Wiley and Sons.

Blaschek, W. (1990). "[Cellulose, an interesting excipient for pharmaceutic use]." Pharm Unserer Zeit 19(2): 73-81.

Bluhm, T. L. and P. Zugenmaier (1981). "Detailed structure of the Vh-amylose-iodine complex: a linear polyiodine chain." Carbohydrate Research 89(1): 1-10.

Bodmeier, R. (1997). "Tableting of coated pellets." European Journal of Pharmaceutics and Biopharmaceutics 43(1): 1-8.

Bolhuis, G. K., H. V. Van Kamp, et al. (1986). "On the Similarity of Sodium Starch Glycolate from Different Sources." Drug Dev Ind Pharm 12(4): 621-630.

Bowker, M. J. (1996). "Analytical challenges in the development of modified-release oral solid dosage forms. A review." Analyst 121(10): 91R-100R.

Braggio, S., R. J. Barnaby, et al. (1996). "A strategy for validation of bioanalytical methods." Journal of Pharmaceutical and Biomedical Analysis 14(4): 375-388.

Brazel, C. S. and N. A. Peppas (2000). "Modeling of drug release from swellable polymers." Eur J Pharm Biopharm 49(1): 47-58. 
Brisson, J., H. Chanzy, et al. (1991). "The crystal and molecular structure of VH amylose by electron diffraction analysis." International Journal of Biological Macromolecules 13(1): 31-39.

Brouillet, F., B. Bataille, et al. (2010). High-amylose sodium carboxymethyl starch sustained release excipient and process for preparing the same. US Patent 20100113619 A1.

Brouillet, F., G. Baylac, et al. (2010). "High-amylose sodium carboxymethyl starch matrices for oral, sustained drug release: development of a spray-drying manufacturing process." Drug Dev Ind Pharm 36(7): 795-805.

Buléon, A., P. Colonna, et al. (1998). "Starch granules: structure and biosynthesis." International Journal of Biological Macromolecules 23(2): 85-112.

Buléon, A., M. M. Delage, et al. (1990). "Single crystals of V amylose complexed with isopropanol and acetone." International Journal of Biological Macromolecules 12(1): 25-33.

Buleon, A., F. Duprat, et al. (1984). "Single crystals of amylose with a low degree of polymerization." Carbohydrate Polymers 4(3): 161-173.

Buri, P. (1987). "Définition et classification des systèmes matriciels." S.T.P. Pharma 3: 193199.

Buri, P. and E. Doelker (1997). Mise à la disposition de l'organisme de principes actifs à partir des formes pharmaceutiques destinées à l'administration par voie orale. Traité de biopharmacie et pharmacocinétique. P.-P. Leblanc, J.-M. Aiache, J.-G. Besner, P. Buri and M. Lesne, Les Presses de l'Université de Montréal: 55-86.

Byers, J. P. and J. G. Sarver (2009). Chapter 10 - Pharmacokinetic Modeling. Pharmacology. H. Miles, M. William, W. M. Kenneth BachmannA2 - Miles Hacker and B. Kenneth. San Diego, Academic Press: 201-277.

Cairns, P., T. Y. Bogracheva, et al. (1997). "Determination of the polymorphic composition of smooth pea starch." Carbohydrate Polymers 32(3-4): 275-282.

Cao, Q. R., Y. W. Choi, et al. (2005). "Formulation, release characteristics and bioavailability of novel monolithic hydroxypropylmethylcellulose matrix tablets containing acetaminophen." J Control Release 108(2-3): 351-361.

Cartilier, L., M. A. Mateescu, et al. (1997). Cross-linked amylose as a binder/disintegrant in tablets, Labopharm, Inc. (Quebec, CA). US Patent 5616343 A.

Cartilier, L., I. Moussa, et al. (1999). Substitude amylose as a matrix for sustained drug release. US Patent 5879707.

Cartilier, L., M. Ungur, et al. (2005). Tablet formulation for sustained drug-release. CA Patent Application 2491665.

Caspary, W. F. (1992). "Physiology and pathophysiology of intestinal absorption." Am J Clin Nutr 55(1 Suppl): 299S-308S.

Chebli, C. and L. Cartilier (2000). "Effect of some physical parameters on the sustained drugrelease properties of substituted amylose matrices." International Journal of Pharmaceutics 193(2): 167-173.

Chebli, C., L. Cartilier, et al. (2001). "Substituted amylose as a matrix for sustained-drug release: a biodegradation study." International Journal of Pharmaceutics 222(2): 183189.

Chebli, C., I. Moussa, et al. (1999). "Substituted amylose as a matrix for sustained drug release." Pharmaceutical Research 16(9): 1436-1440. 
Chien, Y. W. (1982). Fundamentals of controlled drug administration. Novel Drug Delivery System. J. Swarbrick. New York, Marcel Dekker, Inc.: 465-574.

Chin, K.-V., I. Pastan, et al. (1992). Function and Regulation of the Human Multidrug Resistance Gene. Advances in Cancer Research. F. V. W. George and K. George, Academic Press. Volume 60: 157-180.

Code, C. F. and J. A. Marlett (1975). "The interdigestive myo-electric complex of the stomach and small bowel of dogs." J Physiol 246(2): 289-309.

Cohen, J., B. Hubert, et al. (1990). "The Development of USP Dissolution and Drug Release Standards." Pharm Res 7(10): 983-987.

Collett, J. and C. Moreton (2002). Modified-release peroral dosage forms. Pharmaceutics: The Science of Dosage Form Design. M. E. Aulton, Churchill Livingstone: 289-305.

Colombo, P., R. Bettini, et al. (1999). "Drug volume fraction profile in the gel phase and drug release kinetics in hydroxypropylmethyl cellulose matrices containing a soluble drug." European Journal of Pharmaceutical Sciences 9(1): 33-40.

Colombo, P., R. Bettini, et al. (1995). "Drug diffusion front movement is important in drug release control from swellable matrix tablets." Journal of Pharmaceutical Sciences 84(8): 991-997.

Colombo, P., R. Bettini, et al. (1999). "Observation of swelling process and diffusion front position during swelling in hydroxypropyl methyl cellulose (HPMC) matrices containing a soluble drug." Journal of Controlled Release 61(1-2): 83-91.

Colombo, P., R. Bettini, et al. (2000). "Swellable matrices for controlled drug delivery: gellayer behaviour, mechanisms and optimal performance." Pharmaceutical Science \& Technology Today 3(6): 198-204.

Colombo, P., P. Santi, et al. (2000). Drug release from swelling-controlled systems. Handbook of Pharmaceutical Controlled Release Technology. D. L. Wise, A. M. Klibanov, R. Langeret al. New York, Marcel Dekker, Inc.: 183-210.

Cooper, G. M. (2000). Structure of the Plasma Membrane.

Cordon-Cardo, C., J. P. O'Brien, et al. (1990). "Expression of the multidrug resistance gene product (P-glycoprotein) in human normal and tumor tissues." J Histochem Cytochem 38(9): 1277-1287.

Costa, P. and J. M. Sousa Lobo (2001). "Modeling and comparison of dissolution profiles." European Journal of Pharmaceutical Sciences 13(2): 123-133.

Court, M. H., S. X. Duan, et al. (2001). "Interindividual variability in acetaminophen glucuronidation by human liver microsomes: identification of relevant acetaminophen UDP-glucuronosyltransferase isoforms." J Pharmacol Exp Ther 299(3): 998-1006.

Dabbagh, M. A., J. L. Ford, et al. (1999). "Release of propranolol hydrochloride from matrix tablets containing sodium carboxymethylcellulose and hydroxypropylmethylcellulose." Pharm Dev Technol 4(3): 313-324.

Das, N. G. and S. K. Das (2003). "Controlled-Release of Oral Dosage Forms." Pharm.Technol. 27: 10-16.

Daugherty, A. L. and R. J. Mrsny (1999). "Transcellular uptake mechanisms of the intestinal epithelial barrier Part one." Pharmaceutical Science \& Technology Today 2(4): 144151.

Davis, S. S., J. G. Hardy, et al. (1986). "Transit of pharmaceutical dosage forms through the small intestine." Gut 27(8): 886-892. 
Davis, S. S., J. G. Hardy, et al. (1986). "Gastrointestinal transit of a controlled release naproxen tablet formulation." Int J Pharm 32(1): 85-90.

Demirturk, E. and L. Öner (2003). "In Vitro - In Vivo Correlations." Journal of Pharmaceutical Sciences 28: 215-224.

Derendorf, H., L. J. Lesko, et al. (2000). "Pharmacokinetic/pharmacodynamic modeling in drug research and development." J Clin Pharmacol 40(12 Pt 2): 1399-1418.

Devarajan, P. V., P. N. Sule, et al. (1999). "Comparative pharmacodynamic-pharmacokinetic correlation of oral sustained-release theophylline formulation in adult asthmatics." Drug Dev Ind Pharm 25(4): 529-534.

Doelker, E. (1985). Cinétique et mécanismes de libération contrôlée à partir des systèmes polymériques. Formes pharmaceutiques nouvelles, Techniques et Documentation. Paris Technique et Documentation - Lavoisier: 67-134.

Doelker, E. (1990). Swelling behavior of water-soluble cellulose derivatives. Absorbent Polymer Technology. L. Brannon-Peppas and R. Harland. New York, Elsevier: 125145.

Doelker, E. (1993). "Comparative compaction properties of various Microcrystalline Cellulose types and Generic Products." Drug Dev Ind Pharm 19(17-18): 2399-2471.

Doherty, C. and P. York (1989). "Microenvironmental pH control of drug dissolution." Int J Pharm 50(3): 223-232.

Dresse, A., M. A. Gerard, et al. (1978). "Human pharmacokinetics of two crystalline and galenic forms of diflunisal, a new analgesic." Pharm Acta Helv 53(6): 177-181.

Dressman, J. (2005). Pharmaceutical dissolution testing, Taylor \& Francis Group, LLC.

Dressman, J. B., G. L. Amidon, et al. (1998). "Dissolution testing as a prognostic tool for oral drug absorption: immediate release dosage forms." Pharm Res 15(1): 11-22.

Dumoulin, Y., S. Alex, et al. (1998). "Cross-linked amylose as matrix for drug controlled release. X-ray and FT-IR structural analysis." Carbohydrate Polymers 37(4): 361-370.

Dumoulin, Y., F. Carriere, et al. (1998). Manufacture of cross-linked amylose useful as a excipient for control release of active compounds Rougier Inc.

Dumoulin, Y., L. H. Cartilier, et al. (1999). "Cross-linked amylose tablets containing $\alpha-$ amylase: an enzymatically-controlled drug release system." Journal of Controlled Release 60(2-3): 161-167.

Durig, T. and R. Fassihi (2002). "Guar-based monolithic matrix systems: effect of ionizable and non-ionizable substances and excipients on gel dynamics and release kinetics." $\underline{J}$ Control Release 80(1-3): 45-56.

Dutta, S., Y. Qiu, et al. (2005). "Once-a-day extended-release dosage form of divalproex sodium III: development and validation of a Level A in vitro-in vivo correlation (IVIVC)." Journal of Pharmaceutical Sciences 94(9): 1949-1956.

Edge, S. and R. W. Miller (2005). Sodium starch glycolate. Handbook of pharmaceutical excipients. R. C. Rowe, P. J. Sheskey and S. C. Owen. London-Chicago, Pharmaceutical Press: 701-704.

Emami, J. (2006). "In vitro - in vivo correlation: from theory to applications." J Pharm Pharm Sci 9(2): 169-189.

Fang, J. M., P. A. Fowler, et al. (2004). "The chemical modification of a range of starches under aqueous reaction conditions." Carbohydrate Polymers 55(3): 283-289.

Ferrero, C., D. Massuelle, et al. (2010). "Towards elucidation of the drug release mechanism from compressed hydrophilic matrices made of cellulose ethers. II. Evaluation of a 
possible swelling-controlled drug release mechanism using dimensionless analysis." Journal of Controlled Release 141(2): 223-233.

Ferrero, R. C., N. Bruneau, et al. (2000). Hydrophilic cellulose derivatives as drug delivery carriers: the influence of substitution type on the properties of compressed matrix tablets. Handbook of Pharmaceutical Controlled Release Technology. D. L. Wise. New York, Marcel Dekker, Inc.: 1-30.

Ford, J. L., K. Mitchell, et al. (1991). "Hydroxypropylmethylcellulose matrix tablets containing propranolol hydrochloride and sodium dodecyl sulphate." Int J Pharm 71(3): 213-221.

Forrest, J. A., J. A. Clements, et al. (1982). "Clinical pharmacokinetics of paracetamol." Clin Pharmacokinet 7(2): 93-107.

French, D. (1984). Organization of starch granules. New York, Academy Press.

Fry, C. (2007). "Cell physiology I." Surgery (Oxford) 25(10): 401-406.

Gabr, K. E. (1992). "Effect of organic acids on the release patterns of weakly basic drugs from inert sustained release matrix tablets." European Journal of Pharmaceutics and Biopharmaceutics 38: 199-202.

Galia, E., E. Nicolaides, et al. (1998). "Evaluation of various dissolution media for predicting in vivo performance of class I and II drugs." Pharm Res 15(5): 698-705.

Gao, P., J. W. Skoug, et al. (1996). "Swelling of hydroxypropyl methylcellulose matrix tablets. 2. Mechanistic study of the influence of formulation variables on matrix performance and drug release." Journal of Pharmaceutical Sciences 85(7): 732-740.

Germino, F. J. and R. M. Valletta (1964). "Amylose V complexes from dimethyl sulfoxide solutions." Journal of Polymer Science Part A: General Papers 2(11): 4757-4763.

Gernat, C., S. Radosta, et al. (1990). "Supramolecular Structure of Legume Starches Revealed by X-Ray Scattering." Starch - Stärke 42(5): 175-178.

Gidley, M. J. (1989). "Molecular mechanisms underlying amylose aggregation and gelation." Macromolecules 22(1): 351-358.

Giunchedi, P., E. Gavini, et al. (2000 ). "Evaluation of alginate compressed matrices as prolonged drug delivery systems." AAPS PharmSciTech. 1(3): 31-36.

Gladden, L. F. (1994). "Nuclear magnetic resonance in chemical engineering: Principles and applications." Chemical Engineering Science 49(20): 3339-3408.

Glynn-Barnhart, A., M. Hill, et al. (1988). "Sustained release theophylline preparations. Practical recommendations for prescribing and therapeutic drug monitoring." Drugs 35(6): 711-726.

Godet, M. C., A. Buléon, et al. (1993). "Structural features of fatty acid-amylose complexes." Carbohydrate Polymers 21(2-3): 91-95.

Goldenheim, P. D., E. A. Conrad, et al. (1987). "Treatment of asthma by a controlled-release theophylline tablet formulation: a review of the North American experience with nocturnal dosing." Chronobiol Int 4(3): 397-408.

Goldin, B. R. (1990). "Intestinal microflora: metabolism of drugs and carcinogens." Ann Med 22(1): 43-48.

Grond, S. and A. Sablotzki (2004). "Clinical pharmacology of tramadol." Clin Pharmacokinet 43(13): 879-923.

Groschwitz, K. R. and S. P. Hogan (2009). "Intestinal barrier function: Molecular regulation and disease pathogenesis." Journal of Allergy and Clinical Immunology 124(1): 3-20.

Guilbault, A. and C. Mercier (1988). The Polysaccharides. New York, Academic Press. 
Gupta, P. K. and J. R. Robinson (1992). Oral Controlled-Release Delivery. Treatise on Controlled Drug Delivery: Fundamentals, Optimization, Applications. A. Kydonieus. New York, Marcel Dekker, Inc.: 255-314.

Guyot-Hermann, A. M. and D. J. Ringard (1981). "Disintegration Mechanisms of Tablets Containing Starches. Hypothesis About the Particle-Particle Repulsive Force." Drug Dev Ind Pharm 7(2): 155-177.

Hamidi, M., B. Sheikholeslami, et al. (2012). "Lack of evidence for involvement of pglycoprotein in brain uptake of the centrally acting analgesic, tramadol in the rat." J Pharm Pharm Sci 15(5): 606-615.

Hancock, B. C., G. T. Carlson, et al. (2001). "The powder flow and compact mechanical properties of two recently developed matrix-forming polymers." International Journal of Pharmaceutics 53(9): 1193-1199.

Helbert, W. and H. Chanzy (1994). "Single crystals of V amylose complexed with n-butanol or n-pentanol: structural features and properties." International Journal of Biological Macromolecules 16(4): 207-213.

Heller, J. (1980). "Controlled release of biologically active compounds from bioerodible polymers." Biomaterials 1(1): 51-57.

Heller, J. (1987). Use of polymers in controlled release of active agents. Controlled drug delivery: fundamentals and applications. J. R. Robinson and V. H. L. Lee. New York, Marcel Dekker, Inc. 29: 179-211.

Heller, J., R. W. Baker, et al. (1978). "Controlled drug release by polymer dissolution. I. Partial esters of maleic anhydride copolymers - properties and theory." Journal of Applied Polymer Science 22(7): 1991-2009.

Hennink, W. E., S. J. De Jong, et al. (2004). "Biodegradable dextran hydrogels crosslinked by stereocomplex formation for the controlled release of pharmaceutical proteins." Int J Pharm 277(1-2): 99-104.

Herman, J. and J. P. Remon (1989). "Modified starches as hydrophilic matrices for controlled oral delivery. II. In vitro drug release evaluation of thermally modified starches." International Journal of Pharmaceutics 56(1): 65-70.

Herman, J. and J. P. Remon (1990). "Modified starches as hydrophilic matrices for controlled oral delivery III. Evaluation of sustained-release theophylline formulations based on thermal modified starch matrices in dogs." International Journal of Pharmaceutics 63(3): 201-205.

Herman, J., J. P. Remon, et al. (1989,). "Modified starches as hydrophilic matrices for controlled oral delivery. I. Production and characterisation of thermally modified starches." International Journal of Pharmaceutics 56(1): 51-63.

Higuchi, T. (1963). "Mechanism of Sustained-Action Medication. Theoretical Analysis of Rate of Release of Solid Drugs Dispersed in Solid Matrices." Journal of Pharmaceutical Sciences 52: 1145-1149.

Higuchi, W. I., P. K. Lau, et al. (1963). "Polymorphism and drug availability. Solubility relationships in the methylprednisolone system." Journal of Pharmaceutical Sciences 52(2): 150-153.

Hillery, A. M., A. W. Lloyd, et al. (2001). Drug Delivery and Targeting: For Pharmacists and Pharmaceutical Scientists. London, Taylor \& Francis.

Hirtz, J. (1986). "Importance of analytical methods in pharmacokinetic and drug metabolism studies." Biopharm Drug Dispos 7(4): 315-326. 
Hizukuri, S., Y. Takeda, et al. (1981). "Multi-branched nature of amylose and the action of debranching enzymes." Carbohydrate Research 94(2): 205-213.

Ho, R. H. and R. B. Kim (2005). "Transporters and drug therapy: implications for drug disposition and disease." Clin Pharmacol Ther 78(3): 260-277.

Hoener, B.-a. and L. Z. Benet (2002). Factors Influencing Drug Absoption and Drug Availability. Modern Pharmaceutics. G. S. Banker and C. T. Rhodes. New York, Marcel Dekker, Inc.: 145-186.

Hörter, D. and J. B. Dressman (2001). "Influence of physicochemical properties on dissolution of drugs in the gastrointestinal tract." Advanced Drug Delivery Reviews 46(1-3): 75 87.

Hsien-Chih, H. W. and A. Sarko (1978). "The double-helical molecular structure of crystalline a-amylose." Carbohydrate Research 61(1): 27-40.

Huang, X. and C. S. Brazel (2001). "On the importance and mechanisms of burst release in matrix-controlled drug delivery systems." Journal of Controlled Release 73(2-3): 121136.

Hui, H.-W., J. R. Robinson, et al. (1987). Design and fabrication of oral controlled release drug delivery systems. Controlled drug delivery: fundamentals and applications. J. R. Robinson and V. H. L. Lee. New York, Marcel Dekker, Inc. 29: 373-431.

Hummel, T., S. Roscher, et al. (1996). "Assessment of analgesia in man: tramadol controlled release formula vs. tramadol standard formulation." Eur J Clin Pharmacol 51(1): 3138.

Imai, Y., K. Abe, et al. (1990). "Circadian blood pressure variations under different pathophysiological conditions." J Hypertens Suppl 8(7): S125-132.

Ing-Lorenzini, K. R., J. A. Desmeules, et al. (2009). "Two-dimensional liquid chromatography-ion trap mass spectrometry for the simultaneous determination of ketorolac enantiomers and paracetamol in human plasma: Application to a pharmacokinetic study." Journal of Chromatography A 1216(18): 3851-3856.

Ishibashi, T., K. Ikegami, et al. (1999). "Evaluation of colonic absorbability of drugs in dogs using a novel colon-targeted delivery capsule (CTDC)." J Control Release 59(3): 361376.

Jana, S., S. Mandlekar, et al. (2010). "Prodrug design to improve pharmacokinetic and drug delivery properties: challenges to the discovery scientists." Curr Med Chem 17(32): 3874-3908.

Jane, J. L. and J. F. Chen (1992 ). "Effect of Amylose Molecular Size and Amylopectin Branch Chain Length on Paste Properties of Starch." Cereal Chemistry 69(1): 60-65.

Jantzen, G. M. and J. R. Robinson (2002). Sustained- and Controlled-Release Drug Delivery Systems. Modern Pharmaceutics. G. S. Banker and C. T. Rhodes. New York, Marcel Dekker, Inc.: 747-789.

Jivraj, M., L. G. Martini, et al. (2000). "An overview of the different excipients useful for the direct compression of tablets." Pharmaceutical Science \& Technology Today 3(2): 5863.

Johnson, B., H. Greer, et al. (1973). " Rate of dissolution of digoxin tablets as a predictor of absorption." The Lancet 301(7818): 1473-1475.

Jorgensen, E. D. and D. Bhagwat (1998). "Development of dissolution tests for oral extendedrelease products." Pharmaceutical Science \& Technology Today 1(3): 128-135. 
Juliano, R. L. and V. Ling (1976). "A surface glycoprotein modulating drug permeability in Chinese hamster ovary cell mutants." Biochim Biophys Acta 455(1): 152-162.

Kadlec, G. J., L. T. Ha, et al. (1978). "Theophylline half-life in infants and young children." Annals of allergy 40(5): 303-310.

Kamel, S. (2008). "Pharmaceutical significance of cellulose: A review." Express Polymer Letters 2(11): 758-778.

Kanaan, M., Y. Daali, et al. (2009). "Uptake/efflux transport of tramadol enantiomers and Odesmethyl-tramadol: focus on P-glycoprotein." Basic Clin Pharmacol Toxicol 105(3): 199-206.

Katzhendler, I., A. Hoffman, et al. (1997). "Modeling of drug release from erodible tablets." Journal of Pharmaceutical Sciences 86(1): 110-115.

Kazarian, S. G. and J. van der Weerd (2008). "Simultaneous FTIR spectroscopic imaging and visible photography to monitor tablet dissolution and drug release." Pharm Res 25(4): 853-860.

Keating, G. M. (2006). "Tramadol sustained-release capsules." Drugs 66(2): 223-230.

Kellow, J. E., T. J. Borody, et al. (1986). "Human interdigestive motility: variations in patterns from esophagus to colon." Gastroenterology 91(2): 386-395.

Kelly, K. A. (1980). "Gastric emptying of liquids and solids: roles of proximal and distal stomach." Am J Physiol 239(2): G71-76.

Khan, G. M. and Z. Jiabi (1998). "Formulation and in vitro evaluation of ibuprofen-Carbopol 974P-NF controlled release matrix tablets. III: Influence of co-excipients on release rate of the drug." J Control Release 54(2): 185-190.

Khanvilkar, K., M. D. Donovan, et al. (2001). "Drug transfer through mucus." Advanced Drug Delivery Reviews 48(2-3): 173-193.

Khosla, R., L. C. Feely, et al. (1989). "Gastrointestinal transit of non-disintegrating tablets in fed subjects." Int J Pharm 53(2): 107-117.

Khullar, P., R. K. Khar, et al. (1998). "Evaluation of guar gum in the preparation of sustainedrelease matrix tablets." Drug Dev Ind Pharm 24(11): 1095-1099.

Kiang, T. K., M. H. Ensom, et al. (2005). "UDP-glucuronosyltransferases and clinical drugdrug interactions." Pharmacol Ther 106(1): 97-132.

Kokate, A., V. P. Marasanapalle, et al. (2005). Physiological and Biological Barriers to Drug Delivery. Design of Controlled Release Drug Delivery Systems. New York, USA, McGraw-Hill: 42-73.

Kolars, J. C., K. S. Lown, et al. (1994). "CYP3A gene expression in human gut epithelium." Pharmacogenetics 4(5): 247-259.

Kost, J. and S. Shefer (1990). "Chemically-modified polysaccharides for enzymaticallycontrolled oral drug delivery." Biomaterials 11(9): 695-698.

Kottke, M. J. and E. M. Rudnic (2002). Tablet Dosage Forms. Modern Pharmaceutics. G. S. Banker and C. T. Rhodes. New York, Marcel Dekker, Inc.: 437-511.

Kumar, K. P. S., D. Bhowmik, et al. (2010). "Innovations in Sustained Release Drug Delivery System and Its Market Opportunities." Journal of Chemical and Pharmaceutical Research 2(1): 349-360.

Langer, R. S. and D. L. Wise (1984). Medical applications of controlled release.

Le Bail, P., F. G. Morin, et al. (1999). "Characterization of a crosslinked high amylose starch excipient." International Journal of Biological Macromolecules 26(2-3): 193-200. 
Le Hir, A., J.-C. Chaumeil, et al. (2009). Pharmacie galénique: Bonnes pratiques de fabrication des médicaments. Paris.

Leblanc, P.-P., J.-M. Aiache, et al. (1997). Traité de biopharmacie et pharmacocinétique, Les Presses de l'Université de Montréal.

Lee, B.-J., S.-G. Ryu, et al. (1999). "Formulation and Release Characteristics of Hydroxypropyl Methylcellulose Matrix Tablet Containing Melatonin." Drug Dev Ind Pharm 25(4): 493-501.

Lee, P. I. and C.-J. Kim (1991). "Probing the mechanisms of drug release from hydrogels." Journal of Controlled Release 16(1-2): 229-236.

Lee, P. I. and N. A. Peppas (1987). "Prediction of polymer dissolution in swellable controlledrelease systems." Journal of Controlled Release 6(1): 207-215.

Lenaerts, V., Y. Dumoulin, et al. (1991). "Controlled release of theophylline from cross-linked amylose tablets." Journal of Controlled Release 15(1): 39-46.

Lenaerts, V., I. Moussa, et al. (1998). "Cross-linked high amylose starch for controlled release of drugs: recent advances." Journal of Controlled Release 53(1-3): 225-234.

Leppert, W. (2011). "CYP2D6 in the Metabolism of Opioids for Mild to Moderate Pain." Pharmacology 87(5-6): 274-285.

Lewis, K. S. and N. H. Han (1997). "Tramadol: a new centrally acting analgesic." Am J Health Syst Pharm 54(6): 643-652.

Li, V. H. K., J. R. Robinson, et al. (1987). Influence of drug properties and routes of drug administration on the design of sustained and controlled release systems. Controlled drug delivery: fundamentals and applications. J. R. Robinson and V. H. L. Lee. New York, Marcel Dekker, Inc. 29: 3-69.

Lindenbaum, J., V. Butler Jr, et al. (1973). " Correlation of dogoxin-tablet dissolution-rate with biological availability." The Lancet 301(7814): 1215-1217.

Lintz, W., H. Barth, et al. (1998). "Pharmacokinetics of tramadol and bioavailability of enteral tramadol formulations. 3rd Communication: suppositories." Arzneimittelforschung 48(9): 889-899.

Lintz, W., S. Erlacin, et al. (1981). "[Biotransformation of tramadol in man and animal (author's transl)]." Arzneimittelforschung 31(11): 1932-1943.

Long, M. and Y. Chen (2009). Chapter 14 - Dissolution Testing of Solid Products. Developing Solid Oral Dosage Forms. Q. Yihong, C. Yisheng, G. Z. Z. Geoffet al. San Diego, Academic Press: $319-340$.

Loo, J. C. and S. Riegelman (1968). "New method for calculating the intrinsic absorption rate of drugs." Journal of Pharmaceutical Sciences 57(6): 918-928.

Lotfipour, F., A. Nokhodchi, et al. (2004). "The effect of hydrophilic and lipophilic polymers and fillers on the release rate of atenolol from HPMC matrices." Il Farmaco 59(10): 819-825.

Lu, Y., S. Kim, et al. (2011). "In vitro-in vivo correlation: Perspectives on model development." Int J Pharm 418(1): 142-148.

Luner, P. E., S. R. Babu, et al. (1994). "The effects of bile salts and lipids on the physicochemical behavior of gemfibrozil." Pharm Res 11(12): 1755-1760.

MacFarlane, A. J. and P. J. Stover (2008). Gastrointestinal Disorders: Overview. International Encyclopedia of Public Health. H. Editor-in-Chief: Kris. Oxford, Academic Press: 919. 
Maderuelo, C., A. Zarzuelo, et al. (2011). "Critical factors in the release of drugs from sustained release hydrophilic matrices." Journal of Controlled Release 154(1): 2-19.

Majid Khan, G. and J.-B. Zhu (1999). "Studies on drug release kinetics from ibuprofencarbomer hydrophilic matrix tablets: influence of co-excipients on release rate of the drug." Journal of Controlled Release 57(2): 197-203.

Malmud, L. S., R. S. Fisher, et al. (1982). "Scintigraphic evaluation of gastric emptying." Seminars in Nuclear Medicine 12(2): 116-125.

Malonne, H., B. Sonet, et al. (2004). "Pharmacokinetic evaluation of a new oral sustained release dosage form of tramadol." Br J Clin Pharmacol 57(3): 270-278.

Manov, I., Y. Bashenko, et al. (2006). "Involvement of the multidrug resistance Pglycoprotein in acetaminophen-induced toxicity in hepatoma-derived HepG2 and Hep3B cells." Basic Clin Pharmacol Toxicol 99(3): 213-224.

Marieb, E. N. and K. Hoehn (2010). Anatomie et physiologie humaines.

Marvola, M., H. Aito, et al. (1987). "Gastrointestinal Transit and Concomitant Absorption of Verapamil from a Single-Unit Sustained-Release Tablet." Drug Dev Ind Pharm 13(9): 1593-1609.

Mattia, C. and F. Coluzzi (2005). "Tramadol. Focus on musculoskeletal and neuropathic pain." Minerva Anestesiol 71(10): 565-584.

Mattok, G. L. and I. J. McGilveray (1973). "Proceedings: The effect of food intake and sleep on the absorption of acetaminophen." Rev Can Biol 32: 77-84.

Mayersohn, M. (2002). Factors Influencing Drug Absoption and Drug Availability. Modern Pharmaceutics. G. S. Banker and C. T. Rhodes. New York, Marcel Dekker, Inc.: 2366.

McGrane, S. J., D. E. Mainwaring, et al. (2004). "The Role of Hydrogen Bonding in Amylose Gelation." Starch - Stärke 56(3-4): 122-131.

McKenna, A. and D. F. McCafferty (1982). "Effect on particle size on the compaction mechanism and tensile strength of tablets." J Pharm Pharmacol 34(6): 347-351.

McKinnon, R. A., W. M. Burgess, et al. (1995). "Characterisation of CYP3A gene subfamily expression in human gastrointestinal tissues." Gut 36(2): 259-267.

Meibohm, B. and H. Derendorf (1997). "Basic concepts of pharmacokinetic/pharmacodynamic (PK/PD) modelling." Int J Clin Pharmacol Ther 35(10): 401-413.

Melia, C. D. (1991). "Hydrophilic matrix sustained release systems based on polysaccharide carriers." Crit Rev Ther Drug Carrier Syst 8(4): 395-421.

Melia, C. D., N. Washington, et al. (1994). Multiparticulate Controlled Release Oral Dosage Forms: Technology and Biopharmaceutics. Edinburgh, Scottish Academic Press.

Mikac, U., J. Kristl, et al. (2011). "Using quantitative magnetic resonance methods to understand better the gel-layer formation on polymer-matrix tablets." Expert Opinion on Drug Delivery 8(5): 677-692.

Minekus, M., M. Smeets-Peeters, et al. (1999). "A computer-controlled system to simulate conditions of the large intestine with peristaltic mixing, water absorption and absorption of fermentation products." Appl Microbiol Biotechnol 53(1): 108-114.

Moes, A. J. (1993). "Gastroretentive dosage forms." Crit Rev Ther Drug Carrier Syst 10(2): 143-195.

Moghadam, S. H., H. W. Wang, et al. (2007). "Substituted amylose matrices for oral drug delivery." Biomed Mater 2(1): S71-77. 
Moghaddami, M., A. Cummins, et al. (1998). "Lymphocyte-filled villi: Comparison with other lymphoid aggregations in the mucosa of the human small intestine." Gastroenterology 115(6): 1414-1425.

Mojaverian, P., R. K. Ferguson, et al. (1985). "Estimation of gastric residence time of the Heidelberg capsule in humans: effect of varying food composition." Gastroenterology 89(2): 392-397.

Mojaverian, P., J. Rosen, et al. (1997). "In-vivo/in-vitro correlation of four extended release formulations of pseudoephedrine sulfate." Journal of Pharmaceutical and Biomedical Analysis 15(4): 439-445.

Moore, J. W. and H. H. Flanner (1996). "Mathematical comparison of dissolution profiles." Pharm. Tech. 20 (6): 64-74.

Morris, K. R., M. G. Fakes, et al. (1994). "An integrated approach to the selection of optimal salt form for a new drug candidate." Int J Pharm 105(3): 209-217.

Morris, M. E. and G. Levy (1984). "Renal clearance and serum protein binding of acetaminophen and its major conjugates in humans." Journal of Pharmaceutical Sciences 73(8): 1038-1041.

Moussa, I. S. and L. H. Cartilier (1996). "Characterization of moving fronts in cross-linked amylose matrices by image analysis." Journal of Controlled Release 42(1): 47-55.

Moussa, I. S. and L. H. Cartilier (1997). "Evaluation of cross-linked amylose press-coated tablets for sustained drug delivery." International Journal of Pharmaceutics 149(2): 139-149.

Moussa, I. S., V. Lenaerts, et al. (1998). "Image analysis studies of water transport and dimensional changes occurring in the early stages of hydration in cross-linked amylose matrices." Journal of Controlled Release 52(1-2): 63-70.

$\mathrm{Mu}, \mathrm{X} ., \mathrm{M}$. J. Tobyn, et al. (2003). "Development and evaluation of bio-dissolution systems capable of detecting the food effect on a polysaccharide-based matrix system." $\underline{\mathrm{J}}$ Control Release 93(3): 309-318.

Nabais, T., F. Brouillet, et al. (2007). "High-amylose carboxymethyl starch matrices for oral sustained drug-release: In vitro and in vivo evaluation." European Journal of Pharmaceutics and Biopharmaceutics 65(3): 371-378.

Nakano, M., N. Nakazono, et al. (1987). "Preparation and evaluation of sustained release tablets prepared with alpha-starch." Chem Pharm Bull 35(10): 4346-4350.

Nakano, M., N. Ohmori, et al. (1983). "Sustained release of theophylline from hydroxypropylcellulose tablets." Journal of Pharmaceutical Sciences 72(4): 378-380.

Narasimhan, B. and N. A. Peppas (1997). "Molecular analysis of drug delivery systems controlled by dissolution of the polymer carrier." Journal of Pharmaceutical Sciences 86(3): 297-304.

Newman, A. W., R. L. Mueller, et al. (2006). Starches and Starch Derivatives. Encyclopedia of Pharmaceutical Technology, Third Edition. J. Swarbrick: 3476-3482.

Niwa, T., N. Murayama, et al. (2009). "Oxidation of endobiotics mediated by xenobioticmetabolizing forms of human cytochrome." Curr Drug Metab 10(7): 700-712.

Nokhodchi, A., D. Hassan-Zadeh, et al. (2008). "Effect of various surfactants and their concentration on controlled release of captopril from polymeric matrices." Acta Pharm 58(2): 151-162.

Overholser, B. R. and D. R. Foster (2011). "Opioid pharmacokinetic drug-drug interactions." Am J Manag Care 17(11): S276-287. 
Palangio, M., D. W. Northfelt, et al. (2002). "Dose Conversion and Titration with a Novel, Once-Daily, OROS ${ }^{\circledR}$ Osmotic Technology, Extended-Release Hydromorphone Formulation in the Treatment of Chronic Malignant or Nonmalignant Pain." Journal of Pain and Symptom Management 23(5): 355-368.

Park, H. M., S. M. Chernish, et al. (1984). "Gastric emptying of enteric-coated tablets." Dig Dis Sci 29(3): 207-212.

Parker, R. and S. G. Ring (2001). "Aspects of the Physical Chemistry of Starch." Journal of Cereal Science 34(1): 1-17.

Patel, B. N., N. Sharma, et al. (2009). "An accurate, rapid and sensitive determination of tramadol and its active metabolite O-desmethyltramadol in human plasma by LCMS/MS." Journal of Pharmaceutical and Biomedical Analysis 49(2): 354-366.

Peppas, N. A. (1985). "Analysis of Fickian and non-Fickian drug release from polymers." Pharm Acta Helv 60(4): 110-111.

Peppas, N. A. and J. J. Sahlin (1989). "A simple equation for the description of solute release. III. Coupling of diffusion and relaxation." Int J Pharm 57(2): 169-172.

Pérez, S. and E. Bertoft (2010). "The molecular structures of starch components and their contribution to the architecture of starch granules: A comprehensive review." Starch Stärke 62(8): 389-420.

Perkampus, H. H. (1992). UV-Vis Spectroscopy and Its Applications. Berlin, Springer-Verlag.

Pillay, V. and R. Fassihi (1998). "Evaluation and comparison of dissolution data derived from different modified release dosage forms: an alternative method." Journal of Controlled Release 55(1): 45-55.

Pillay, V. and R. Fassihi (2000). "A novel approach for constant rate delivery of highly soluble bioactives from a simple monolithic system." Journal of Controlled Release 67(1): 6778.

Prajapati, V. D., G. K. Jani, et al. (2013). "Raft forming system—An upcoming approach of gastroretentive drug delivery system." Journal of Controlled Release 168(2): 151-165.

Proudfoot, S. (2002). Dosage regimens. Pharmaceutics: The Science of Dosage Form Design. M. E. Aulton, Churchill Livingstone: 275-288.

Qiu, Y. (2009). Chapter 17 - In Vitro-In Vivo Correlations: Fundamentals, Development Considerations, and Applications. Developing Solid Oral Dosage Forms. Q. Yihong, C. Yisheng, G. Z. Z. Geoffet al. San Diego, Academic Press: 379-406.

Qiu, Y., H. Cheskin, et al. (1997). "Sustained-release hydrophilic matrix tablets of zileuton: formulation and in vitro/in vivo studies." Journal of Controlled Release 45(3): 249256.

Qiu, Y., J. Garren, et al. (2003). "Once-a-day controlled-release dosage form of divalproex sodium II: development of a predictive in vitro drug release method." Journal of Pharmaceutical Sciences 92(11): 2317-2325.

Raber, M., H. U. Schulz, et al. (1999). "Pharmacokinetic properties of tramadol sustained release capsules. 3rd communication: investigation of relative bioavailability under steady state conditions." Arzneimittelforschung 49(7): 594-598.

Radbruch, L., S. Grond, et al. (1996). "A risk-benefit assessment of tramadol in the management of pain." Drug Saf 15(1): 8-29.

Raffa, R. B., E. Friderichs, et al. (1992). "Opioid and nonopioid components independently contribute to the mechanism of action of tramadol, an 'atypical' opioid analgesic." J Pharmacol Exp Ther 260(1): 275-285. 
Raffa, R. B., R. K. Nayak, et al. (1995). "The mechanism(s) of action and pharmacokinetics of tramadol hydrochloride." Rev Contemp Pharmacother 6: 485-497.

Raghavendra Rao, N. G., G. Sagar, et al. (2009). "Formulation and evaluation of sustained release matrix tablets of tramadol hydrochloride " International Journal of Pharmacy and Pharmaceutical Sciences 1(1 ): 60-70.

Rahmouni, M., V. Lenaerts, et al. (2002). "Influence of physical parameters and lubricants on the compaction properties of granulated and non-granulated cross-linked high amylose starch." Chem Pharm Bull 50(9): 1155-1162.

Rajabi-Siahboomi, A. R. (2003). "An overview of current oral modified release technologies." Business Briefing: Pharmatech 1: 181-184.

Rajan, A., V. S. Prasad, et al. (2006). "Enzymatic esterification of starch using recovered coconut oil." International Journal of Biological Macromolecules 39(4-5): 265-272.

Ranade, V. V. (1991). "Drug delivery systems 5A. Oral drug delivery." J Clin Pharmacol 31(1): 2-16.

Ranade, V. V. (1991). "Drug delivery systems 5A. Oral drug delivery." The Journal of Clinical Pharmacology 31(1): 2-16.

Read, N. W., M. N. Al-Janabi, et al. (1986). "Simultaneous measurement of gastric emptying, small bowel residence and colonic filling of a solid meal by the use of the gamma camera." Gut 27(3): 300-308.

Read, N. W., C. A. Miles, et al. (1980). "Transit of a meal through the stomach, small intestine, and colon in normal subjects and its role in the pathogenesis of diarrhea." Gastroenterology 79(6): 1276-1282.

Read, N. W. and K. Sugden (1988). "Gastrointestinal dynamics and pharmacology for the optimum design of controlled-release oral dosage forms." $\underline{\text { Crit Rev Ther Drug Carrier }}$ Syst 4(3): 221-263.

Rekhi, G. S., R. V. Nellore, et al. (1999). "Identification of critical formulation and processing variables for metoprolol tartrate extended-release (ER) matrix tablets." Journal of Controlled Release 59(3): 327-342.

Rencher, W. F., S. Babu, et al. (1995). Sustained release matrix system using hydroxyethyl cellulose and hydroxypropyl cellulose polymer blends. US Patent 5451409 A.

Richardson, J. C., R. W. Bowtell, et al. (2005). "Pharmaceutical applications of magnetic resonance imaging (MRI)." Advanced Drug Delivery Reviews 57(8): 1191-1209.

Roškar, R. and T. T. Lušin (2012). Analytical Methods for Quantification of Drug Metabolites in Biological Samples.

Sajeev, C. and R. N. Saha (2001). "Formulation and comparative evaluation of controlled release diclofenac tablets prepared by matrix-embedding technique, membrane barrier technique, and combination of the two." Drug Development Research 53(1): 1-8.

Sarna, S. K. (1985). "Cyclic motor activity; migrating motor complex: 1985." Gastroenterology 89(4): 894-913.

Saša, B., P. Odon, et al. (2006). "Analysis of surface properties of cellulose ethers and drug release from their matrix tablets." European Journal of Pharmaceutical Sciences 27(4): 375-383.

Sathe, P. M., Y. Tsong, et al. (1996). "In-vitro dissolution profile comparison: statistics and analysis, model dependent approach." Pharm Res 13(12): 1799-1803. 
Schuetz, E. G., W. T. Beck, et al. (1996). "Modulators and substrates of P-glycoprotein and cytochrome P4503A coordinately up-regulate these proteins in human colon carcinoma cells." Mol Pharmacol 49(2): 311-318.

Serajuddin, A. T. M. (2007). "Salt formation to improve drug solubility." Advanced Drug Delivery Reviews 59(7): 603-616.

Shah, U. and L. Augsburger (2002). "Multiple Sources of Sodium Starch Glycolate, NF: Evaluation of Functional Equivalence and Development of Standard Performance Tests." Pharmaceutical Development and Technology 7(3): 345-359.

Shah, V. P., J. J. Konecny, et al. (1989). "In vitro dissolution profile of water-insoluble drug dosage forms in the presence of surfactants." Pharm Res 6(7): 612-618.

Shah, V. P., Y. Tsong, et al. (1998). "In vitro dissolution profile comparison--statistics and analysis of the similarity factor, f2." Pharm Res 15(6): 889-896.

Shameem, M., N. Katori, et al. (1995). "Oral solid controlled release dosage forms: role of GImechanical destructive forces and colonic release in drug absorption under fasted and fed conditions in humans." Pharm Res 12(7): 1049-1054.

Shargel, L., S. Wu-Pong, et al. (2004). Bioavailability and bioequivalence Applied Biopharmaceutics \& Pharmacokinetics, McGraw-Hill Medical: 453-499.

Shargel, L., S. Wu-Pong, et al. (2004). Biopharmaceutical considerations in drug product design. Applied Biopharmaceutics \& Pharmacokinetics, McGraw-Hill Medical: 411452.

Shargel, L., S. Wu-Pong, et al. (2004). Introduction to biopharmaceutics and pharmacokinetics. Applied Biopharmaceutics \& Pharmacokinetics, McGraw-Hill Medical: 1-19.

Shargel, L., S. Wu-Pong, et al. (2004). Mathematical fundamentals in pharmacokinetics Applied Biopharmaceutics \& Pharmacokinetics, McGraw-Hill Medical: 21-50.

Shargel, L., S. Wu-Pong, et al. (2004). Multicompartment models: intravenous bolus administration. Applied Biopharmaceutics \& Pharmacokinetics, McGraw-Hill Medical: 73-105.

Shargel, L., S. Wu-Pong, et al. (2004). One-compartment open model: intravenous bolus administration. Applied Biopharmaceutics \& Pharmacokinetics, McGraw-Hill Medical: 51-72.

Shaw, T. R., K. Raymond, et al. (1973). "Therapeutic non-equivalence of digoxin tablets in the United Kingdom: correlation with tablet dissolution rate." Br Med J 4(5895): 763 766.

Shimada, T., H. Yamazaki, et al. (1994). "Interindividual variations in human liver cytochrome P-450 enzymes involved in the oxidation of drugs, carcinogens and toxic chemicals: studies with liver microsomes of 30 Japanese and 30 Caucasians." J Pharmacol Exp Ther 270(1): 414-423.

Short, R. W. P. and F. Verbanac (1971). Compressed tablets containing compacted starch as binder-disintegrant ingredient. US Patent $3622677 \mathrm{~A}$.

Siepe, S., B. Lueckel, et al. (2006). "Strategies for the design of hydrophilic matrix tablets with controlled microenvironmental pH." Int J Pharm 316(1-2): 14-20.

Siepe, S., B. Lueckel, et al. (2008). "Assessment of tailor-made HPMC-based matrix minitablets comprising a weakly basic drug compound." Drug Dev Ind Pharm 34(1): 46-52. 
Siepmann, J. and N. A. Peppas (2001). "Modeling of drug release from delivery systems based on hydroxypropyl methylcellulose (HPMC)." Advanced Drug Delivery Reviews 48(23): 139-157.

Simó, C., A. Cifuentes, et al. (2003). "Drug delivery systems: polymers and drugs monitored by capillary electromigration methods." Journal of Chromatography B 797(1-2): 37 49.

Slanar, O., M. Nobilis, et al. (2007) "Pharmacokinetics of tramadol is affected by MDR1 polymorphism C3435T", Eur J Clin Pharmacol. Apr;63(4):419-21. Epub 2007 Jan 30.

Sloan, J. W., C. L. Mehltretter, et al. (1962). "Carboxymethyl High-Amylose Starch." Journal of Chemical \& Engineering Data 7(1): 156-158.

Smith, D. A. and B. C. Jones (1992). "Speculations on the substrate structure-activity relationship (SSAR) of cytochrome P450 enzymes." Biochem Pharmacol 44(11): 2089-2098.

Smith, H. J. and M. Feldman (1986). "Influence of food and marker length on gastric emptying of indigestible radiopaque markers in healthy humans." Gastroenterology 91(6): 1452-1455.

Soltero, R. A., J. M. Hoover, et al. (1989). "Effects of sinker shapes on dissolution profiles." Journal of Pharmaceutical Sciences 78(1): 35-39.

Stella, V. J. and K. W. Nti-Addae (2007). "Prodrug strategies to overcome poor water solubility." Adv Drug Deliv Rev 59(7): 677-694.

Subrahmanyam, V., A. B. Renwick, et al. (2001). "Identification of cytochrome P-450 isoforms responsible for cis-tramadol metabolism in human liver microsomes." Drug Metab Dispos 29(8): 1146-1155.

Sumathi, S. and A. R. Ray (2002). "Release behaviour of drugs from tamarind seed polysaccharide tablets." Journal of Pharmacy \& Pharmaceutical Sciences 5(1): 12-18.

Susumu Hizukuri, Y. T., Michiko Yasuda (1981). "Multi-branched nature of amylose and the action of debranching enzymes." Carbohydrate Research 94(2): 205-213.

Svensson, E. and A.-C. Eliasson (1995). "Crystalline changes in native wheat and potato starches at intermediate water levels during gelatinization." Carbohydrate Polymers 26(3): 171-176.

Takeda, Y., N. Maruta, et al. (1992). "Examination of the structure of amylose by tritium labelling of the reducing terminal." Carbohydrate Research 227(0): 113-120.

Talukdar, M. M., A. Michoel, et al. (1996). "Comparative study on xanthan gum and hydroxypropylmethyl cellulose as matrices for controlled-release drug delivery I. Compaction and in vitro drug release behaviour." Int J Pharm 129(1-2): 233-241.

Talukdar, M. M. and J. Plaizier-Vercammen (1993). "Evaluation of xanthan gum as a hydrophillic matrix for controlled release dosage form preparations." Drug Dev Ind Pharm. 19(9): 1037-1046.

Te Wierik, G. H. P., J. Bergsma, et al. (1996). "A new generation of starch products as excipient in pharmaceutical tablets. I. Preparation and binding properties of high surface area potato starch products." International Journal of Pharmaceutics 134(1-2): 27-36.

Te Wierik, G. H. P., A. C. Eissens, et al. (1997). "A new generation of starch products as excipient in pharmaceutical tablets. II. High surface area retrograded pregelatinized potato starch products in sustained-release tablets." Journal of Controlled Release 45(1): 25-33. 
Te Wierik, G. H. P., A. C. Eissens, et al. (1993). "Preparation, characterization and pharmaceutical application of linear dextrins. I. Preparation and characterization of amylodextrin, metastable amylodextrins, and metastable amylose." Pharmaceutical Research 10(9): 1274-1279.

Te Wierik, G. H. P., A. C. Eissens, et al. (1994). "Preparation, characterization and pharmaceutical application of linear dextrins: V. Study on the binding properties of amylodextrin, metastable amylodextrin and metastable amylose." International Journal of Pharmaceutics 102(1-3): 81-90.

Te Wierik, G. H. P., J. Van der Veen, et al. (1993). "Preparation, characterization and application of linear dextrins. Part VI. General applicability and mechanism of programmed release from amylodextrin tablets." Journal of Controlled Release 27(1): 9-17.

Tester, R. F., J. Karkalas, et al. (2004). "Starch—composition, fine structure and architecture." Journal of Cereal Science 39(2): 151-165.

Thérien-Aubin, H., W. E. Baille, et al. (2005). "Imaging of High-Amylose Starch Tablets. 3. Initial Diffusion and Temperature Effects." Biomacromolecules 6(6): 3367-3372.

Thewlis, B. H. and S. Albans (1969). "Carboxymethyl Wheat Starch." Starch - Stärke 21(1): 21-24.

Thiebaut, F., T. Tsuruo, et al. (1987). "Cellular localization of the multidrug-resistance gene product P-glycoprotein in normal human tissues." Proceedings of the National Academy of Sciences 84(21): 7735-7738.

Thompson, D. G., D. L. Wingate, et al. (1980). "Normal patterns of human upper small bowel motor activity recorded by prolonged radiotelemetry." Gut 21(6): 500-506.

Thomson, A. B. and J. M. Dietschy (1977). "Derivation of the equations that describe the effects of unstirred water layers on the kinetic parameters of active transport processes in the intestine." J Theor Biol 64(2): 277-294.

Thummel, K. E., K. L. Kunze, et al. (1997). "Enzyme-catalyzed processes of first-pass hepatic and intestinal drug extraction." Advanced Drug Delivery Reviews 27(2-3): 99-127.

Tijsen, C. J., H. J. Kolk, et al. (2001). "An experimental study on the carboxymethylation of granular potato starch in non-aqueous media." Carbohydrate Polymers 45(3): 219-226.

Tijsen, C. J., H. J. Scherpenkate, et al. (1999). "Optimisation of the process conditions for the modification of starch." Chemical Engineering Science 54(13-14): 2765-2772.

Tijsen, C. J., R. M. Voncken, et al. (2001). "Design of a continuous process for the production of highly substituted granular carboxymethyl starch." Chemical Engineering Science 56(2): 411-418.

Tran, P. H., H. T. Tran, et al. (2008). "Modulation of microenvironmental pH and crystallinity of ionizable telmisartan using alkalizers in solid dispersions for controlled release." $\underline{\mathrm{J}}$ Control Release 129(1): 59-65.

Trubiano, P. C. (1983). Low swelling starches as tablet disintegrants. US Patent 4369308.

Tsong, Y., T. Hammerstrom, et al. (1996). "Statistical Assessment of Mean Differences between Two Dissolution Data Sets*." Drug Information Journal 30(4): 1105-1112.

$\mathrm{Tu}$, J., Y. Shen, et al. (2011). Polymers in Oral Modified Release Systems. Oral Controlled Release Formulation Design and Drug Delivery: Theory to Practice. H. Wen and K. Park, John Wiley \& Sons: 71-88.

Ungur, M., L. Cartilier, et al. (2004). Formulation de comprimé pour libération soutenue de principe actif. Maîtrise, Université de Montréal. 
Ungur, M., N. Yonis, et al. (2005). The evaluation of carboxymethylamylose for oral drug delivery systems: from laboratory to pilot scale. Montreal, Canada, Books of Abstracts of the ISAB2-2005 3rd International Symposium on Advanced Biomaterials/Biomechanics: 271.

Uppoor, V. R. S. (2001). "Regulatory perspectives on in vitro (dissolution)/in vivo (bioavailability) correlations." Journal of Controlled Release 72(1-3): 127-132.

Urso, R. and L. Aarons (1983). "Bioavailability of drugs with long elimination half-lives." Eur J Clin Pharmacol 25(5): 689-693.

Urso, R., P. Blardi, et al. (2002). "A short introduction to pharmacokinetics." European Review for Medical and Pharmacological Sciences 6: 33-44.

van Aerde, P. and J. P. Remon (1988). "In vitro evaluation of modified starches as matrices for sustained release dosage forms." International Journal of Pharmaceutics 45(1-2): 145152.

Varma, M. V., S. Khandavilli, et al. (2004). "Biopharmaceutic classification system: a scientific framework for pharmacokinetic optimization in drug research." Curr Drug Metab 5(5): 375-388.

Varshosaz, J., N. Tavakoli, et al. (2006). "Use of hydrophilic natural gums in formulation of sustained-release matrix tablets of tramadol hydrochloride." 7(1): E168-E174.

Vlase, L., S. E. Leucuta, et al. (2008). "Determination of tramadol and O-desmethyltramadol in human plasma by high-performance liquid chromatography with mass spectrometry detection." Talanta 75(4): 1104-1109.

Wacher, V. J., L. Salphati, et al. (2001). "Active secretion and enterocytic drug metabolism barriers to drug absorption." Advanced Drug Delivery Reviews 46(1-3): 89-102.

Wacher, V. J., C. Y. Wu, et al. (1995). "Overlapping substrate specificities and tissue distribution of cytochrome P450 3A and P-glycoprotein: implications for drug delivery and activity in cancer chemotherapy." Mol Carcinog 13(3): 129-134.

Wagner, D., H. Spahn-Langguth, et al. (2001). "Intestinal drug efflux: formulation and food effects." Advanced Drug Delivery Reviews 50, Supplement 1(0): S13-S31.

Wagner, J. G. (1983). "Pharmacokinetic absorption plots from oral data alone or oral/intravenous data and an exact loo-riegelman equation." Journal of Pharmaceutical Sciences 72(7): 838-842.

Wagner, J. G. and E. Nelson (1963). "Per cent absorbed time plots derived from blood level and/or urinary excretion data." Journal of Pharmaceutical Sciences 52: 610-611.

Wagner, J. G. and E. Nelson (1964). "Kinetic analysis of blood levels and urinary excretion in the absorptive phase after single doses of drug." Journal of Pharmaceutical Sciences 53(11): 1392-1403.

Wang, T. L., T. Y. Bogracheva, et al. (1998). "Starch: as simple as A, B, C?" Journal of Experimental Botany 49(320): 481-502.

Wang, Y. J., E. Assaad, et al. (2011). "NMR imaging of chitosan and carboxymethyl starch tablets: Swelling and hydration of the polyelectrolyte complex." Int J Pharm 419(1-2): 215-221.

Washington, N., C. Washington, et al. (2001). Physiological Pharmaceutics: Barriers to Drug Absorption, Taylor and Francis Inc. 
Watkins, P. B., S. A. Wrighton, et al. (1987). "Identification of glucocorticoid-inducible cytochromes P-450 in the intestinal mucosa of rats and man." J Clin Invest 80(4): 1029-1036.

Welling, P. G. and M. R. Dobrinska (1987). Dosing considerations and bioavailability assessment of controlled drug delivery systems. Controlled drug delivery: fundamentals and applications. J. R. Robinson and V. H. L. Lee. New York, Marcel Dekker, Inc. 29: 253-291.

Whittam, M. A., P. D. Orford, et al. (1989). "Aqueous dissolution of crystalline and amorphous amylose-alcohol complexes." International Journal of Biological Macromolecules 11(6): 339-344.

Wurzburg, O. B. (1972). Starch in the food industry. Handbook of Food Additives. T. E. Furia. Cleveland, CRC Press: 361-395.

Xu, C., C. Y. Li, et al. (2005). "Induction of phase I, II and III drug metabolism/transport by xenobiotics." Arch Pharm Res 28(3): 249-268.

York, P. (2002). The design of dosage forms. Pharmaceutics: The Science of Dosage Form Design. M. E. Aulton, Churchill Livingstone: 1-12.

Yuksel, N., A. E. Kanık, et al. (2000). "Comparison of in vitro dissolution profiles by ANOVA-based, model-dependent and -independent methods." Int J Pharm 209(1-2): 57-67.

Zaghloul, I. Y. and M. A. Radwan (1997). "High Performance Liquid Chromatographic Determination of Tramadol in Pharmaceutical Dosage Forms." Journal of Liquid Chromatography \& Related Technologies 20(5): 779-787.

Zahirul, M. and I. Khan (1996). "Dissolution testing for sustained or controlled release oral dosage forms and correlation with in vivo data: Challenges and opportunities." International Journal of Pharmaceutics 140(2): 131-143.

Zahirul, M. and I. Khan (1996). "Dissolution testing for sustained or controlled release oral dosage forms and correlation with in vivo data: Challenges and opportunities." Int J Pharm 140(2): 131-143.

Zhang, Y. and L. Z. Benet (2001). "The gut as a barrier to drug absorption: combined role of cytochrome P450 3A and P-glycoprotein." Clin Pharmacokinet 40(3): 159-168.

Zobel, H. F. (1988). "Molecules to Granules: A Comprehensive Starch Review." Starch Stärke 40(2): 44-50. 


\section{Chapter 2. Objectives}

\subsection{General objective}

Oral solid dosage forms that prolong the drug-release rate have proven to be advantageous in the delivery of certain drugs, improving their therapeutic efficacy while preventing the occurrence of toxic and/or subtherapeutic plasma drug concentrations. Biodegradable and abundant hydrophilic excipients, such as modified starches, are interesting excipients for sustained drug-release. SD HASCA was proposed as an innovating hydrophilic excipient for oral SR matrix tablets produced by direct compression. Previous studies involving this new excipient focused on the development and optimization of the production of SD HASCA and the study of formulation parameters. However, further studies aimed at the development and optimization of SD HASCA SR matrix tablets in view of their ultimate use as dosage forms for oral administration, were necessary to demonstrate the usefulness of this polymer for the production of efficient and advantageous sustained drug-release delivery systems.

The general objective of this thesis was to develop, optimize and evaluate in vitro and in vivo SR matrix tablets for oral administration using SD HASCA as the only sustained drugrelease excipient.

\subsection{Specific objectives}

In the first place, the drug-release properties of different formulations containing a compressed blend of acetaminophen, sodium chloride and SD HASCA were evaluated in a series of dissolution media simulating the $\mathrm{pH}$ gradient in the gastrointestinal and its variations in the presence of food. The research performed in this section aimed to: 
1) Select an optimized formulation taking into account the best SR profiles of the formulations tested and their physical integrity when immersed in the simulated gastrointestinal $\mathrm{pH}$ gradient.

2) Study the influence of simulated fasting and food-induced gastric $\mathrm{pH}$ values and residence times in each acidic $\mathrm{pH}$ on the drug-release characteristics of the optimized SD HASCA formulation.

Following the selection of the most appropriate SD HASCA SR formulation, an exploratory clinical study in healthy human volunteers was carried out. The objectives of this section of the study were to:

1) Explore the aptitude of this formulation to extend the absorption of drug after oral administration.

2) Evaluate the resistance of this formulation to mechanical stresses and hydrolysis by $\alpha$ amylase in the gastrointestinal tract.

This clinical study confirmed that SD HASCA matrix tablets are able to extend drugrelease and absorption and that the structure of the swollen matrices is strong enough for oral administration.

The following work focused on development of SD HASCA SR formulations with clinical relevance. The specific objectives of this part of the research were to:

1) Develop once-daily and twice-daily SD HASCA tablets containing two common dosages of tramadol hydrochloride (100 mg and $200 \mathrm{mg}$ ), a model drug with clinical relevance in sustained drug-release.

2) Assess the robustness and drug-release characteristics of one of the developed formulations in a $40 \%$ ethanol medium, since tramadol hydrochloride may lead to side effects if released as a bolus.

3) Investigate the influence of compression force (CF) on the in vitro drug-release rate. 
Subsequently, the research focused on studying in vitro the influence of the most pertinent physiological condition of the gastrointestinal tract taking into account the amylosebased nature of SD HASCA, i.e., biodegradation by $\alpha$-amylase enzymes, on the presystemic metabolism of SD HASCA SR matrix tablets. It was also hypothesized that that variations in gastric residence time of non-disintegrating SD HASCA SR tablets may affect their $\alpha$ amylase-catalyzed erosion. Thus, the final section of the research aimed to:

1) Investigate in vitro the influence of $\alpha$-amylase on the drug-release characteristics and mechanisms of SD HASCA SR formulations.

2) Evaluate in vitro the influence of the residence time in acidic medium on the $\alpha$ amylase-catalyzed erosion of SD HASCA SR formulations.

3) Investigate the contribution of the Fickian diffusion and the erosion mechanisms to the overall drug-release rate from SD HASCA SR formulations through the application of a two-component model, with and without $\alpha$-amylase. 


\section{Chapter 3. High-amylose starch matrices for oral sustained drug-release: in vitro and in vivo evaluation}

Domingues Nabais T. ${ }^{1}$, Brouillet F. ${ }^{1,2}$, Kyriacos S. ${ }^{3}$, Mroueh M. ${ }^{3}$, Amores da Silva P. ${ }^{4}$, Bataille B. ${ }^{2}$, Chebli C. ${ }^{1,5}$ and Cartilier L. ${ }^{1, *}$

${ }^{1}$ Faculty of Pharmacy, University of Montreal, Montreal (Quebec) Canada

${ }^{2}$ Faculty of Pharmacy UMR CIRAD 016, Université Montpellier 1, Montpellier, France

${ }^{3}$ School of Pharmacy, Lebanese American University, Byblos, Lebanon

${ }^{4}$ Health Sciences Department, Universitade Lusofona, Lisbon, Portugal

${ }^{5}$ Present address: Pharmascience Inc., Montreal (Quebec) Canada

* Corresponding author: Faculty of Pharmacy, University of Montreal, Montreal (Quebec)

Canada

Keywords: Drug delivery; sustained release; excipient; polymer; tablet; matrix; starch; amylose; in vitro; in vivo 


\subsection{Presentation of the article and contribution of the authors}

This article presents the results obtained during the development and optimization of a SD HASCA SR formulation containing acetaminophen as the model drug, and sodium chloride as the swollen matrix-stabilizing agent. The first part addresses the study of the influence of acidic $\mathrm{pH}$ value or acidic medium residence time on the in vitro drug-release from an optimized SD HASCA formulation. Thereafter, the results of the clinical study are reported. The citation for this article is:

Nabais, T., F. Brouillet, et al. (2007). "High-amylose carboxymethyl starch matrices for oral sustained drug-release: In vitro and in vivo evaluation." European Journal of Pharmaceutics and Biopharmaceutics 65(3): 371-378.

The contribution of each author to the publication was the following: Teresa Nabais - Execution of all dissolution testing; analysis of data resulting from dissolution testing; analysis of data resulting from the clinical study in healthy volunteers; writing of the article, except introduction.

Fabien Brouillet - Spray drying of the SD HASCA used to manufacture the tablets used in the study; technical support during the first experiments using the dissolution and other apparatuses.

Soula Kyriacos - Execution of clinical studies.

Mohammad Mroueh - Coordinator of the work of Soula Kyriacos.

Pedro Amores da Silva - Co-director of this thesis at the time of the publication; revision of the article.

Bernard Bataille - Project coordinator.

Chafic Chebli - Project coordinator.

Louis Cartilier - Main project coordinator and director at the time of the publication; writing of the introduction of the article; revision of the article. 


\subsection{Abstract}

High-amylose corn starch, that contains $70 \%$ of amylose chains and $30 \%$ of amylopectin, has been used to obtain substituted amylose (SA) polymers. Tablets have been prepared by direct compression, i.e. dry mixing of drug and SA, followed by compression, which is the easiest way to manufacture an oral dosage form. Until now, their controlledrelease properties have been assessed only by an in vitro dissolution test. Amylose-based polymers are normally subject to biodegradation by $\alpha$-amylase enzymes present in the gastrointestinal tract, but matrix systems show no significant degradation of tablets by $\alpha$ amylase in vitro.

High-amylose sodium carboxymethyl starch (HASCA) is an interesting excipient for sustained drug-release in solid oral dosage forms. In addition to the easy manufacturing of tablets by direct compression, the results show that in vitro drug-release from an optimized HASCA formulation is not affected by either acidic $\mathrm{pH}$ value or acidic medium residence time. In addition, a compressed blend of HASCA with an optimized quantity of sodium chloride provides a pharmaceutical SR tablet with improved integrity for oral administration. In vivo studies demonstrate extended drug absorption, showing that the matrix tablets do not disintegrate immediately and resist enzymatic attack well. Nevertheless, acetaminophen does not seem to be the most appropriate drug for this type of formulation.

\subsection{Introduction}

It is possible to chemically modify the hydroxyl groups of amylose by an etherification process, resulting in substituted amylose (SA) $[1,2]$. These polymers are referred to as SA,R- $n$, where $\mathrm{R}$ defines the substituent used, typically 1,2-epoxypropanol (or glycidol=G), and $n$ represents the degree of substitution (DS) expressed as the mole ratio of substituent $/ \mathrm{kg}$ of amylose. High-amylose corn starch, that contains $70 \%$ of amylose chains and $30 \%$ of amylopectin (Hylon VII ${ }^{\circledR}$, Eurylon), has been employed to obtain SA polymers. SA matrix tablets have been prepared by direct compression, i.e. dry mixing of drug and SA,G- $n$, 
followed by compression, which is the easiest way to manufacture an oral dosage form. Their controlled-release properties have been assessed by an in vitro dissolution test. Release times of $95 \%$ of the drug ranged from 9 to 20 hours for all DSs studied for 400 -mg matrices containing $10 \%$ of a soluble drug [2].

Drug transport analysis has revealed that diffusion, relaxation, molecular rearrangement and, in some cases, physical erosion, are simultaneously involved in the control of drug release. SA,G-2.7 matrices allow nearly constant drug-release [2-4]. Such a release profile is unusual for a hydrophilic matrix system where Fickian release, i.e. first-order kinetics, is expected. SA hydrophilic matrix tablets sequentially present a burst effect, typical of hydrophilic matrices, and near-constant release, typical of reservoir systems. After the burst effect, the surface pores disappear progressively by the molecular association of amylose chains; this allows the creation of a polymer layer acting as a diffusion barrier and explains the peculiar behaviour of SA polymers [5].

When testing their in vitro resistance to $\alpha$-amylase enzymatic degradation, SA,G-2.7 matrix systems and dry-coated tablets maintain their structure, and control the release of $\left[{ }^{186} \mathrm{Re}\right]$, showing no significant degradation of tablets by $\alpha$-amylase [6].

High-amylose sodium carboxymethyl starch (HASCA) has been recently proposed as a suitable material for oral matrix tablets [7,8]. These tablets can be advantageously improved by the addition of electrolytes. Such addition permits the integrity of the swollen matrix tablets to be maintained when they are immersed in a medium undergoing $\mathrm{pH}$ changes simulating the $\mathrm{pH}$ evolution of the environment surrounding an oral pharmaceutical dosage form transiting along the gastrointestinal tract while allowing controlled and sustained drug-release with a remarkable close-to-linear release profile [8].

Establishing a meaningful relationship between in vitro drug-release and in vivo absorption from an extended-release dosage form is an important part of the dosage form development process, the ultimate goal of in vitro-in vivo correlation (IVIVC) ideally being the application of in vitro data as a surrogate for in vivo evaluation [9]. However, it must be understood that "IVIVC is not only a drug-dependent characteristic; it is also a productdependent characteristic" [9]. Therefore, the Food and Drug Administration has strictly limited IVIVC conditions of use, thereby avoiding overenthusiastic applications [10]. 
The purpose of this study was: 1) to optimize the concentration of sodium chloride in a formulation that maintains the integrity of swollen HASCA matrix tablets; 2) to evaluate the in vitro drug-release characteristics of an optimized SR formulation containing HASCA, sodium chloride and acetaminophen, employing a $\mathrm{pH}$ gradient to simulate various $\mathrm{pH}$ conditions found in the gastrointestinal tract; 3) to demonstrate the extended-release properties of this new drug delivery system in vivo by comparing the pharmacokinetic parameters of a SA formulation and a commercially-available, immediate-release tablet in healthy volunteers under fasting conditions; and 4) to elaborate the relationship between in vitro release and in vivo absorption, if any, for this specific SR acetaminophen formulation among healthy volunteers under fasting conditions.

\subsection{Material and methods}

\subsubsection{Materials}

HASCA, where "HAS" means high-amylose substituted starch and "CA" defines the substituent used, herein chloroacetate, was obtained from Amylose Project Inc. (Beaconsfield, Quebec, Canada). Acetaminophen was purchased from Laboratoires Denis Giroux inc. (SteHyacinthe, Quebec, Canada), and sodium chloride (crystals, lab grade), from Anachemia Ltd. (Montreal, Quebec, Canada). Commercially-available, immediate-release Tylenol$^{\circ}$ regular- $^{-}$ strength tablets containing $325 \mathrm{mg}$ of acetaminophen, from McNeil (Guelph, Ontario, Canada), were chosen as the reference formulation. All chemicals were of reagent grade and were tested without further purification.

\subsubsection{Preparation of HASCA tablets}

Tablets were prepared by direct compression, i.e. dry mixing of acetaminophen, HASCA, and $\mathrm{NaCl}$, followed by compression. HASCA, acetaminophen and $\mathrm{NaCl}$ were mixed manually in a mortar. No lubricant was added to the formulation. Indeed, it has been 
demonstrated earlier that magnesium stearate, at standard levels, does not influence the in vitro release profile from HASCA matrix tablets containing $\mathrm{NaCl}$ as well as their integrity [8]. Tablets were prepared on an IR 30-ton press (C-30 Research \& Industrial Instruments Company, London, U.K.). To evaluate the $\mathrm{NaCl}$ percentage in the formulation that maintains the integrity of the swollen matrix, tablets of $600 \mathrm{mg}$ containing $40 \%$ of acetaminophen as a model drug and $17.5 \%, 22.5 \%$ or $27.5 \%$ of $\mathrm{NaCl}$, were prepared. On the other hand, $600-\mathrm{mg}$ tablets containing $40 \%$ of drug and $27.5 \%$ of $\mathrm{NaCl}$ were produced to investigate the influence of acidic $\mathrm{pH}$ value and residence time in the acidic medium on the release characteristics of HASCA tablets as well as to perform in vivo studies. All tablets were compressed at 2.5 tons $/ \mathrm{cm}^{2}$ for 20 seconds. The diameter of the tablets was $1.26 \mathrm{~cm}$.

\subsubsection{Drug-release studies}

The drug-release properties of some typical HASCA matrix tablets were assessed by an in vitro dissolution test. Since HASCA is an ionic polymer used for oral, sustained drugrelease, the in vitro release experiments were conducted in a $\mathrm{pH}$ gradient simulating the $\mathrm{pH}$ evolution of the gastrointestinal tract to examine the $\mathrm{pH}$-dependency of drug-release properties. The tablets were placed individually in $900 \mathrm{~mL}$ of a hydrochloric acid medium $(\mathrm{pH}$ 1.2) simulating gastric $\mathrm{pH}$, at $37^{\circ} \mathrm{C}$, in U.S.P. XXIII dissolution apparatus No. 2 equipped with a rotating paddle $(50 \mathrm{rpm})$. They were then transferred to a phosphate buffer medium ( $\mathrm{pH} \mathrm{6.8)}$

simulating jejunum $\mathrm{pH}$, then finally transferred to another phosphate buffer medium ( $\mathrm{pH} 7.4)$ simulating ileum $\mathrm{pH}$, until the end of the test. The dissolution apparatus and all other experimental conditions remained the same.

The standard $\mathrm{pH}$ gradient conditions (A) were: $\mathrm{pH} 1.2$ for 1 hour, $\mathrm{pH} 6.8$ for 3 hours, and $\mathrm{pH} 7.4$ until the end of the dissolution test ( 24 hours). The influence of $\mathrm{NaCl}$ percentage in the formulation on swollen matrix tablet integrity and drug-release was studied under standard conditions. To investigate the influence of $\mathrm{pH}$ of the acidic medium and residence time in the acidic medium on drug-release from HASCA matrices, different dissolution tests were performed, in which both $\mathrm{pH}$ of the acidic medium and residence time in the acidic medium were varied, as shown in Table 3.1. 
Table $3.1 \mathrm{pH}$ gradient conditions used to simulate various possibilities of gastric $\mathrm{pHs}$ and residence times encountered by HASCA matrices during their gastrointestinal transit

\begin{tabular}{|l|lllllc|}
\hline \multicolumn{1}{|c|}{ pH value } & \multicolumn{1}{|c}{$\mathbf{1 . 2}$} & $\mathbf{3 . 5}$ & $\mathbf{4 . 5}$ & $\mathbf{6 . 8}$ & $\mathbf{7 . 4}$ \\
\hline Conditions A (standard) & $1 \mathrm{hr}$ & - & - & $3 \mathrm{hrs}$ & Until 24 hrs \\
Conditions B & $10 \mathrm{~min}$ & - & - & $3 \mathrm{hrs}$ & Until 24 hrs \\
Conditions C & - & $3 \mathrm{hrs}$ & - & $3 \mathrm{hrs}$ & Until 24 hrs \\
Conditions D & - & - & $6 \mathrm{hrs}$ & $3 \mathrm{hrs}$ & Until 24 hrs \\
Conditions E & - & - & - & $3 \mathrm{hrs}$ & Until 24 hrs \\
\hline
\end{tabular}

The amount of acetaminophen released at predetermined time intervals was followed spectrophotometrically $(244 \mathrm{~nm})$. All formulations were tested in triplicate. The drug- release results are expressed as cumulative $\%$ in function of time (hours).

\subsubsection{Pharmacokinetics in healthy human volunteers}

The pharmacokinetic study, carried out at Notre-Dame de Secours Hospital, Byblos, Lebanon, was conducted in 5 healthy volunteers ( 3 males and 2 females) of mean age $22.6 \pm$ 1.8 years and mean weight $82.4 \pm 24.4 \mathrm{~kg}$. All subjects had a normal medical history, medication history, physical examination, and biochemical screening (haematology, blood urea nitrogen, serum creatinine, alkaline phosphatase, liver enzymes, and glucose). None of them had taken any enzyme-inducing agents for at least 30 days prior to the initiation of this study. They were also instructed to abstain from medications and alcohol for at least 1 week prior to and during the study period. Informed consent was obtained from all subjects after the nature and purpose of the study were explained to them, along with a brief description of the drug effect. 
A single-dose, non-blinded, balanced, 2-way crossover design was adopted using 2 acetaminophen dosing arms: a commercially-available, immediate-release (IR) dosage form (Tylenol ${ }^{\circ}$ containing $325 \mathrm{mg}$ of acetaminophen per tablet) and a SR (SR) formulation comprising $240 \mathrm{mg}$ of acetaminophen per tablet (Amylose Project Inc.). All subjects were fasted overnight, and in the morning, each of them was given a single oral dose of either a SR acetaminophen tablet or an IR Tylenol tablet, as a reference, with $240 \mathrm{~mL}$ of water. A standardized lunch or snack was served 4 hours after drug administration. A one week washout period was allowed before the next treatment to avoid potential carry-over effects. Blood samples $(9 \mathrm{~mL})$ were collected, in heparinised plastic tubes, at different time intervals for each of the 2 products: at 0 (blank), $0.25,0.5,1,1.5,2,3,4,6,8,12$ and 24 hours postdosing for Tylenol', and at 0 (blank), 0.25, 0.5, 1, 1.5, 2, 3, 4, 6, 8, 12, 17, 24 and 36 hours post-dosing for the SR dosage form. The blood samples were then centrifuged for 5 minutes at $10,000 \mathrm{rpm}$, and plasma samples stored in a freezer $\left(-70^{\circ} \mathrm{C}\right)$ until analysis.

\subsubsection{High-performance liquid chromatography (HPLC) analysis of plasma samples}

Acetaminophen plasma concentrations were analyzed by HPLC. The HPLC system consisted of a pump (LC-10AD, Shimadzu), a UV-VIS spectrophotometric detector (SPD10AVP, Shimadzu) operating at a wavelength of $254 \mathrm{~nm}$, a Rheodyne injector, a reverse-phase column $(\mathrm{C} 18,15 \mathrm{~cm} \times 4.6 \mathrm{~mm})$ and an integrator (C-R 8A Chromatopac, Shimadzu). The mobile phase, a mixture of water and acetonitrile $(94: 6 \mathrm{v} / \mathrm{v} \%)$, was pumped at a flow rate of $1.2 \mathrm{~mL} / \mathrm{min}$. The lower detection limit of the assay was $0.01 \mu \mathrm{g} / \mathrm{mL}$ of acetaminophen in plasma.

Frozen plasma samples were allowed to reach room temperature. A $200-\mu \mathrm{L}$ aliquot of plasma was placed into a test tube (Eppendorf), to which $70 \mu \mathrm{L}$ of internal standard (theophylline aqueous solution $50 \mu \mathrm{g} / \mathrm{mL}$ for high concentrations [>0.35 $\mu \mathrm{g} / \mathrm{mL}$ ] and $5 \mu \mathrm{g} / \mathrm{mL}$ for low concentrations $[\leq 0.35 \mu \mathrm{g} / \mathrm{mL}]$ ) and $430 \mu \mathrm{L}$ of $10 \%$ perchloric acid were added. The mixture was vigorously shaken for 5 minutes, then centrifuged for another 5 minutes at 10,000 
$\mathrm{rpm}$. The supernatant was finally collected, and $50 \mu \mathrm{L}$ of the filtrate was injected into the HPLC system to record either peak areas or heights.

\subsubsection{Pharmacokinetic data analysis}

Since the 2 formulations presented different doses of acetaminophen, the drug plasma concentrations obtained after Tylenol administration were dose-adjusted, i.e. divided by the acetaminophen dose in the Tylenol formulation ( $325 \mathrm{mg}$ ) and multiplied by the acetaminophen dose in the SR formulation $(240 \mathrm{mg}$ ), before calculation of the pharmacokinetic parameters. Further weight adjustment was performed before plotting the drug concentration-time curves to take into account the high weight variation of the volunteers enrolled in the study.

Maximum plasma concentration $\left(C_{\max }\right)$ and time to reach $C_{\max }\left(T_{\max }\right)$ after oral administration were directly determined from the plasma concentration values. The measured plasma concentrations served to calculate the area under the plasma concentration-time curve from time zero to the last concentration time point $\left(\mathrm{AUC}_{0-\mathrm{t}}\right)$ and the area under the plasma concentration-time curve from time zero to infinity $\left(\mathrm{AUC}_{0-\mathrm{x}}\right)$. $\mathrm{AUC}_{0-\mathrm{t}}$ was calculated according to the trapezoidal rule, and $\mathrm{AUC}_{0-\infty}$ was calculated by the sum of $\mathrm{AUC}_{0-\mathrm{t}}$ and the last measured concentration divided by the elimination constant $\left(C_{t} / k\right)$.

The Wagner-Nelson method [11] was adopted to calculate the percentages of acetaminophen absorbed in function of time for each volunteer enrolled in the study. The absorbed percentages were calculated by the following equation:

$\%$ Absorbed $=\left[\left(C_{\mathrm{t}}+k \times \mathrm{AUC}_{0-\mathrm{t}}\right) /\left(k \times \mathrm{AUC}_{0-\mathrm{-}}\right)\right] \times 100$

where $C_{\mathrm{t}}$ is the plasma concentration at time $\mathrm{t} ; k$ is the elimination rate constant; $\mathrm{AUC}_{0-\mathrm{t}}$ is the area under the plasma concentration-time curve from time zero to time $t$, and $\mathrm{AUC}_{0-x}$ is the area under the plasma concentration-time curve from time zero to infinity. 
All the pharmacokinetic parameters were determined for each volunteer, and the averages, standard deviations and coefficients of variation calculated.

No statistical analysis was performed on any of the pharmaceutical parameters because this exploratory study was conducted on a very small number of volunteers to evaluate the in vitro and in vivo potential of a new SR matrix formulation.

\subsubsection{IVIVC}

An IVIVC for this specific acetaminophen SR formulation, if any, was explored by plotting the point-to-point relationship of the percentage of drug absorbed in vivo vs. the percentage of drug released in vitro at the same time, up to the maximum amount of drug absorbed in vivo.

\subsection{Results and discussion}

\subsubsection{In vitro drug-release characteristics from HASCA matrix tablets}

Figure 3.1 shows the in vitro release profile of acetaminophen from HASCA SR matrix tablets containing $\mathrm{NaCl}$ in concentrations ranging from 17.5 to $27.5 \%$ w/w. Remarkably, all total release times were close to 24 hours for $600-\mathrm{mg}$ tablets containing such large amounts of soluble materials, i.e. around two-thirds w/w of each tablet were composed of acetaminophen and $\mathrm{NaCl}$. 


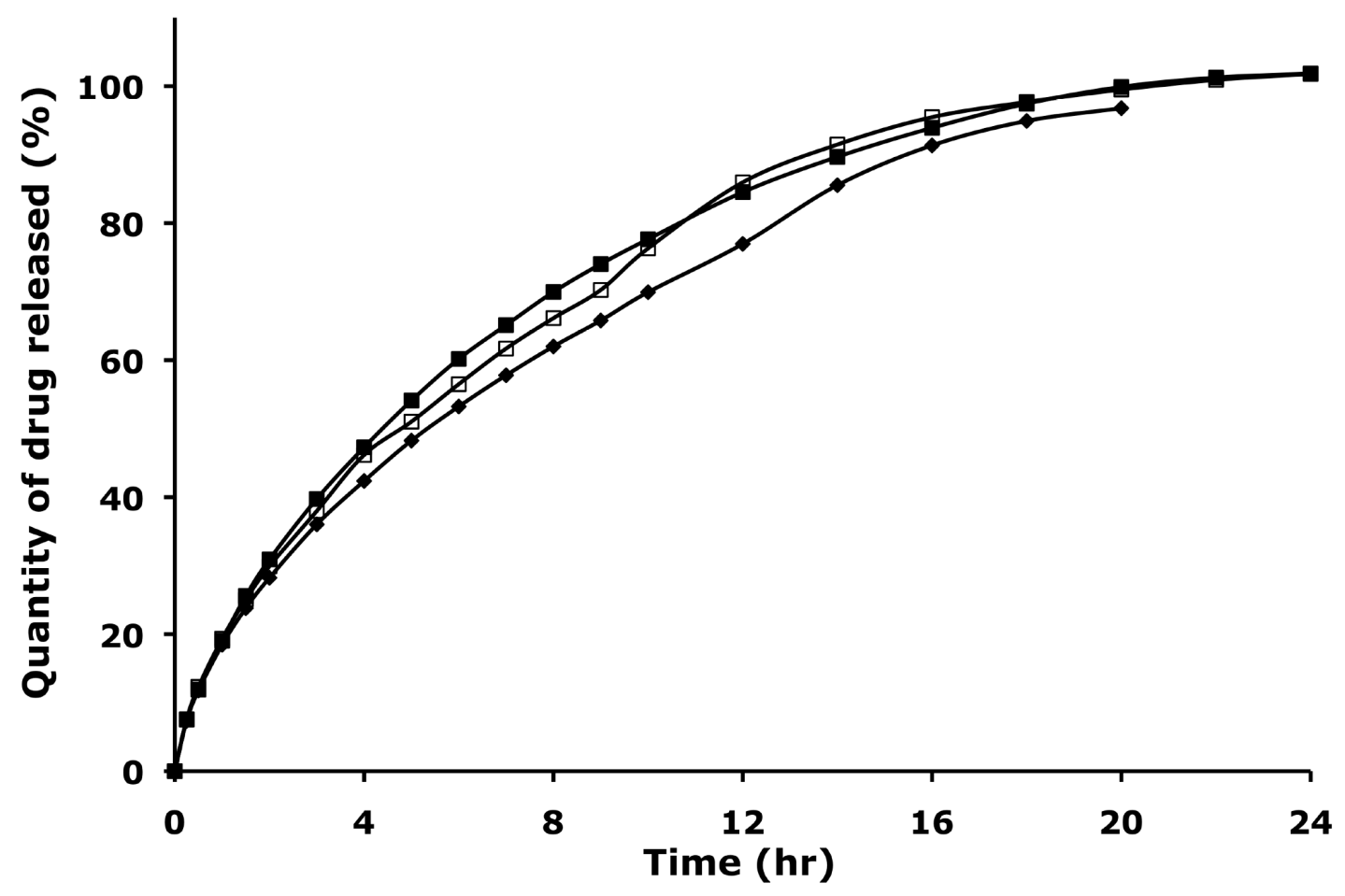

Figure 3.1 Cumulative percent of acetaminophen released in vitro from HASCA matrices containing $40 \%$ of drug and increasing concentrations of $\mathrm{NaCl}$ ( $: 17.5 \%$ of $\mathrm{NaCl}, \square: 22.5 \%$ of $\mathrm{NaCl}, \mathbf{\square}: 27.5 \%$ of $\mathrm{NaCl}$ ). The number of replicates was three.

Further, an increase in $\mathrm{NaCl}$ tablet content did not lead to an increment in the bursteffect. Only after 2 hours of dissolution could a slight rise in the drug-release rate be observed with escalating $\mathrm{NaCl}$ concentrations. However, 9 to 12 hours after the beginning of the dissolution process, some cracks appeared on the surface of the tablets containing 17.5 or $22.5 \% \mathrm{NaCl}$, leading, at the same time, to an increase in the drug-release rate. This can be explained by an augmentation in the tablet diffusion surface as well as by new drug particles being directly exposed to the medium for free dissolution, creating a kind of reduced-scale burst-effect. Even if these cracks did not lead to a dramatic change in the drug-release rate (Fig. 3.1), good mechanical resistance to stress is essential for normal therapeutic use. Indeed, when the stomach churns, thereby exerting significant physical force on the formulation, there is a strong risk of the tablet breaking apart, which could elicit a burst of drug-release. The optimal amount of $\mathrm{NaCl}$ found to maintain the integrity of the swollen tablets during the totality of the dissolution test was $27.5 \%$ for tablets containing $40 \%$ of acetaminophen. It has been previously reported that adding an optimal percentage of electrolyte to the formulation 
could maintain the integrity of swollen hydrophilic matrix tablets while allowing controlled and sustained drug-release [8].

The swelling of SA tablets can be described as moderate when compared to the usual hydrophilic matrices such as hydroxypropylmethylcellulose [5]. This is particularly true for HASCA tablets, which form a very rigid gel with surprisingly high mechanical strength in the swollen state $[7,8]$. As a consequence, it is possible to create tablets that do not show any disintegration, even if mechanical stresses occur, for example, after administration in the gastrointestinal tract. However, in the absence of an electrolyte, such swollen HASCA tablets show cracks and/or partial or complete separation of the tablet parts, which forbid their normal and safe therapeutic use. One possible explanation for the appearance of cracks on the surface of the tablets may be that the rigidity of the gel and its tight network hinder water penetration into the tablets but, more importantly, strongly decrease diffusion of the dissolved drug out of the matrix tablets, thereby tremendously increasing internal osmotic pressure, which in the end produces cracks and/or partial or complete separation of the tablet parts to relieve the tablets internal stress. Figure 3.1 shows that the addition of an electrolyte like $\mathrm{NaCl}$ to the HASCA formulation can provide complete stabilization of the swollen matrix structure or at least significantly delay apparition of the above-mentioned problems and/or decrease their intensity. It is believed that adding an appropriate amount of electrolytes maintains a delicate equilibrium between a) hydrogen bonds created, among others, through $-\mathrm{COOH}$ associations, which enhance gel strength and maintain matrix structure, and b) swelling of the polymer chains, increased by their repulsion due to the $-\mathrm{COO}^{-}$groups, which gives the matrix its necessary elasticity. The picture is further complicated by other factors, which combine their effects to produce complex behaviour: the effect of porosity after dissolution of the drug and the electrolyte on gel structure; the effect of $\mathrm{NaCl}$ on gel viscosity and, therefore, on drug diffusion; the effect of an intramatrix buffer system on gel structure and all other factors affecting it, such as the nature and concentration of the drug, electrolytes and other excipients, and the $\mathrm{pH}$ conditions of the external medium. These results were the opposite of what were expected as, indeed, adding an electrolyte to the formulation should pump more water and faster inside the tablet, thereby increasing internal osmotic pressure and making the tablet present cracks. 


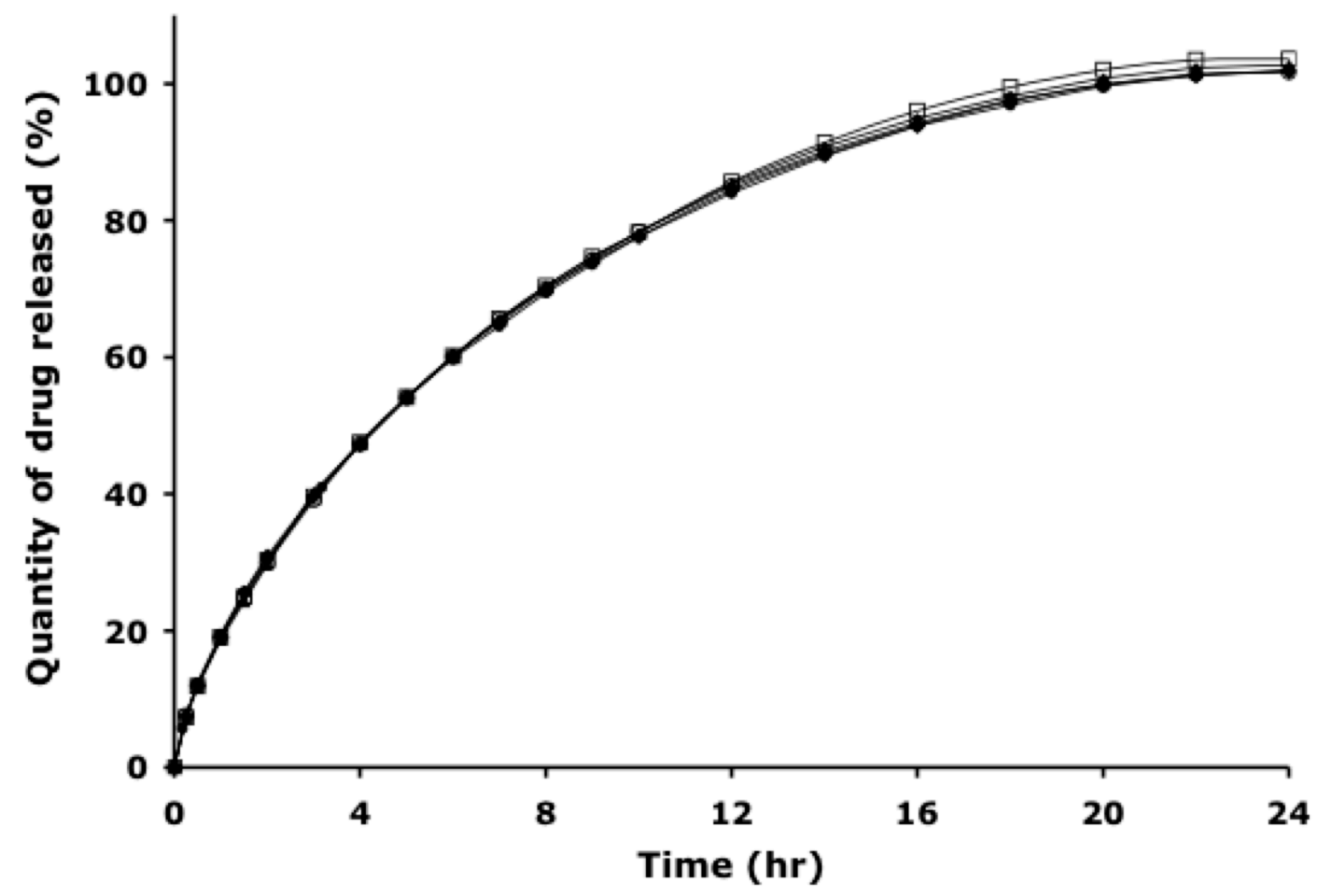

Figure 3.2 Cumulative percentage of acetaminophen released in vitro from optimized HASCA matrices $(32.5 \%$ of HASCA, $40 \%$ of acetaminophen, and $27.5 \%$ of $\mathrm{NaCl})$ in $\mathrm{pH}$ gradients with different acidic $\mathrm{pH}$ conditions $(\mathbf{O}: 10 \mathrm{~min}$ at $\mathrm{pH} 1.2, \diamond: 1 \mathrm{hr}$ at $\mathrm{pH} 1.2, \square: 3 \mathrm{hrs}$ at $\mathrm{pH} 3.5, \mathbf{\Delta}$ : $6 \mathrm{hrs}$ at $\mathrm{pH} 4.5$, O: no acidic medium). The number of replicates was three.

Since HASCA is an ionic polymer used for oral, sustained drug-release, the in vitro release experiments were conducted in a $\mathrm{pH}$ gradient simulating the $\mathrm{pH}$ evolution of the gastrointestinal tract to study the $\mathrm{pH}$-dependency of the release properties. Because of the ionic nature of the HASCA polymer, one would expect immersion in changing $\mathrm{pH}$ environments to result in differences in the behaviour of the formulation when in contact with the various $\mathrm{pH}$ mediums, which could lead to a $\mathrm{pH}$-dependent release rate. Fig. 3.2 shows the release profiles from HASCA SR matrix tablets containing $27.5 \%$ of $\mathrm{NaCl}$ in $\mathrm{pH}$ gradients where both acidic $\mathrm{pH}$ value and residence time in the acidic medium varied according to Table 3.1. No significant differences in the drug-release profiles were observed. The drug-release profile from such SR matrix tablets appears to be independent of both the acidic $\mathrm{pH}$ value and residence time in the acidic medium. Most probably, the combined presence of $\mathrm{NaCl}$ and the carboxylic groups of the polymer creates a buffered environment in the matrix, allowing it to 
be insensitive to $\mathrm{pH}$ changes of the surrounding medium. This is important considering that materials that possess $\mathrm{pH}$-independent release properties are preferable for oral extendedrelease formulations, since they are not affected by intra- and inter-subject variations of both gastric $\mathrm{pH}$ and gastrointestinal transit time [12]. It is expected that this observation will remain true as long as the ionization and solubility of other ingredients in the formulation are not significantly affected by $\mathrm{pH}$ changes in the gastrointestinal tract, as is the case with acetaminophen $\left(p K_{a} 9.5\right)[13]$.

\subsubsection{Pharmacokinetics in healthy human volunteers}

The model drug chosen was acetaminophen, a commonly used analgesic and antipyretic, which is well absorbed by passive diffusion with high permeability throughout the intestinal tract [14]. According to the Biopharmaceutics Classification System [15], acetaminophen is classified as a "class I" drug. Its solubility in water varies from sparingly soluble to soluble at $37^{\circ} \mathrm{C}$, with $\mathrm{pH}$-independent solubility at physiological values (from 1 to 8.5). Hence, this drug is not considered to ionize in the physiological $\mathrm{pH}$ range ( $\left.p K_{a} 9.5\right)$ [13]. The plasma elimination half-life $\left(t_{1 / 2}\right)$ of acetaminophen ranges from 1 to 4 hours [16]. Moreover, the drug is safe and non-toxic when doses ranging from 200 to $400 \mathrm{mg}$ are administered. Therefore, the chosen dose, i.e. $240 \mathrm{mg}$, did not represent any risk of toxicity to the subjects. In addition, acetaminophen is not subject to any significant enzymatic or bacterial degradation in the gastrointestinal lumen. Food reduces the rate of acetaminophen absorption but not its extent [17]. Finally, acetaminophen is easily detected, quantified and relatively inexpensive. Considering that this was an exploratory in vivo study to evaluate a new drugdelivery system, it was important to avoid the usual contraindications of a SR dosage form, i.e., the risk of dose dumping, as a consequence of tablet rupture or erosion, and accumulation in the body. Acetaminophen, at that dosage strength, was thus a safe choice as a model drug. Because of the above-mentioned characteristics, the controlled release of acetaminophen from numerous formulations has been widely investigated [18-20].

The mean drug plasma concentration-time profiles following oral administration of the HASCA SR formulation and the commercial IR tablet are illustrated in Figure 3.3. The 
absence of a burst-effect during the time range between 5 minutes and 2 hours after administration of the HASCA SR matrix tablets to fasted, healthy volunteers, which coincides with normal gastric residence times for a dosage form given in a fasted state [21], suggests that the gel was strong enough to resist the powerful peristaltic contractions normally occurring in the fasted state and which open the pylorus and clear the stomach of any residual material (sometimes called housekeeper waves). Therefore, the in vivo concentration-time profiles show that the tablets maintain their integrity while traversing the stomach. No bursteffect was also detected during the first 3 to 5 hours after administration of the formulation. Considering that residence time in the small intestine is about 3 hours [21], this was the period of time where the HASCA polymer was under attack by $\alpha$-amylase. This absence of bursteffect whilst the dosage form traverses the small intestine indicates that the HASCA polymer resisted $\alpha$-amylase biodegradation.

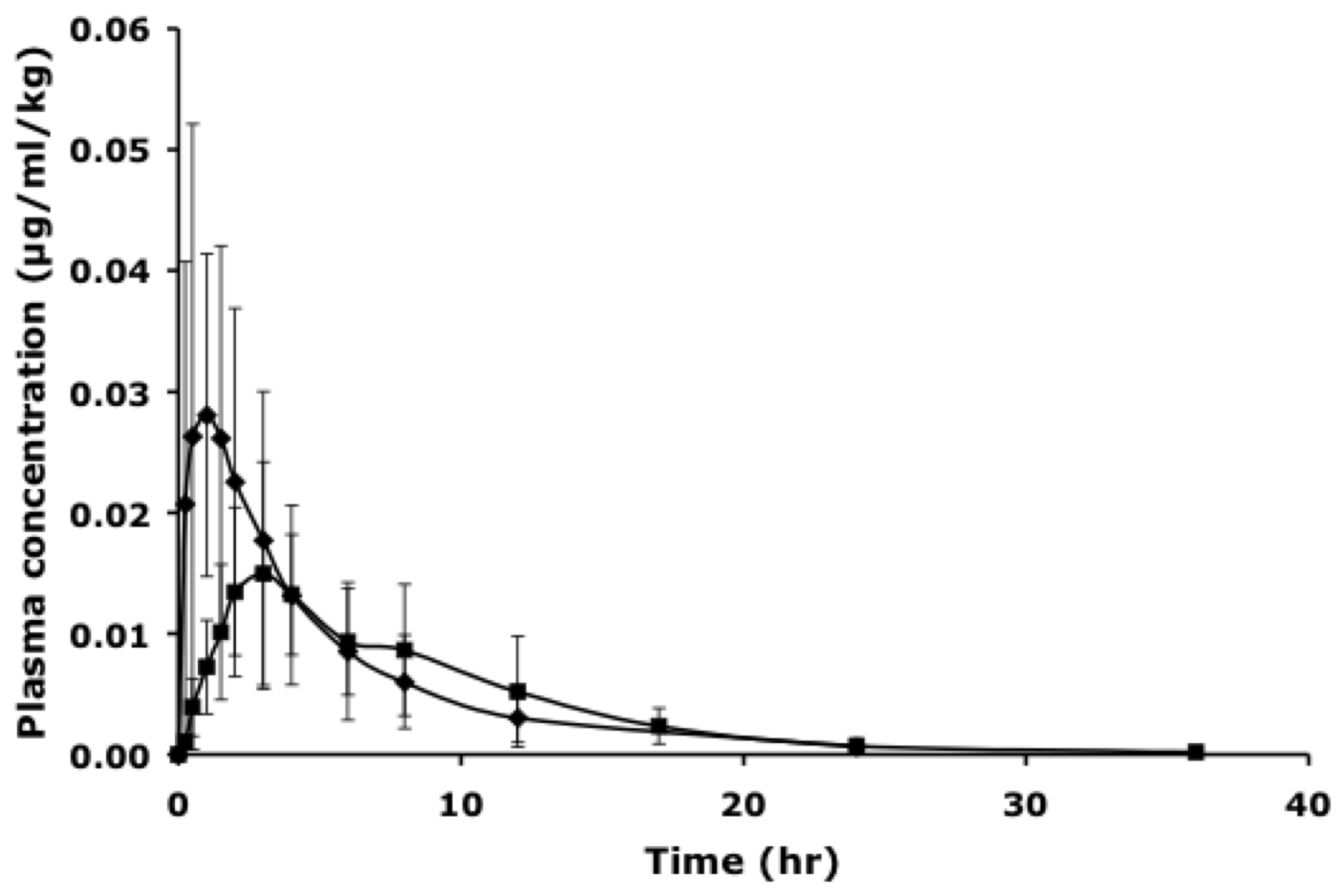

Figure 3.3 Acetaminophen plasma concentration (expressed in $\mu \mathrm{g} / \mathrm{mL} / \mathrm{kg}$ ) vs. time profiles (mean $\pm \mathrm{SD}$ ) following oral administration of the HASCA SR formulation (ם) and the commercial IR tablet $(\diamond)$. 
There were large differences in plasma concentrations between the IR and the SR dosage forms. Differences in the curves reflected different release rates of the 2 formulations. The first portion of the plasma concentration-time curves (Fig. 3.3) showed large differences, demonstrating IR for the Tylenol formulation and a SR profile for the HASCA formulation. The mean ( \pm standard deviation) pharmacokinetic parameters and their respective coefficients of variation $(\% \mathrm{CV})$ are listed in Table 3.2. The very rapid drug absorption from the IR tablets was confirmed by the $C_{\max }$ and $T_{\max }$ values, i.e. $2.90 \mu \mathrm{g} / \mathrm{mL}$ and $45 \mathrm{~min}$ respectively. The AUCs calculated for HASCA tablets and for Tylenol after dose-adjustment were similar, which suggests that the total amount of drug absorbed by the body after administration of the same dose was the same for both formulations, irrespective of the absorption rate.

Table 3.2 Pharmacokinetic parameters of acetaminophen (mean \pm SD and $\% \mathrm{CV}$ ) following oral administration of the HASCA SR formulation and the IR tablet.

\begin{tabular}{|l|cccc|}
\hline & $\begin{array}{c}\mathbf{A U C}_{\mathbf{0 - 2 4}} \mathbf{h r} \\
(\boldsymbol{\mu g} \times \mathbf{h r} \mathbf{m L})\end{array}$ & $\begin{array}{c}\mathbf{A U C}_{\mathbf{0}-\infty} \\
(\boldsymbol{\mu g} \times \mathbf{h r} / \mathbf{m L})\end{array}$ & $\begin{array}{c}\boldsymbol{C}_{\max } \\
(\boldsymbol{\mu} \mathbf{g} / \mathbf{m L})\end{array}$ & $\begin{array}{c}\boldsymbol{T}_{\max } \\
(\mathbf{h r})\end{array}$ \\
\hline$\underline{\text { Tylenol}}$ & & & & \\
Mean $\pm \mathrm{SD}$ & $11.59 \pm 3.13$ & $11.76 \pm 3.20$ & $2.90 \pm 0.97$ & $0.75 \pm 0.50$ \\
$\% \mathrm{CV}$ & 27.01 & 27.24 & 33.37 & 66.67 \\
\hline$\underline{\text { HASCA }}$ & & & & \\
$\underline{\text { matrix tablet }}$ & & & & \\
Mean $\pm \mathrm{SD}$ & $11.14 \pm 3.45$ & $11.24 \pm 3.46$ & $1.32 \pm 0.46$ & $4.40 \pm 2.51$ \\
$\% \mathrm{CV}$ & 30.96 & 30.82 & 34.95 & 57.05 \\
\hline
\end{tabular}

There was relatively high inter-subject variability of the estimated pharmacokinetic parameters, particularly for $T_{\max }$. A possible explanation for the relatively high inter-subject variation of the estimated pharmacokinetic parameters is given by the large differences in anatomic characteristics of the volunteers, like their weight, especially for such a small number of subjects. 
Figure 3.4 shows the average cumulative percentage of drug absorbed in function of time (hours), calculated by the Wagner-Nelson method. Since the $k$ value in the WagnerNelson calculation has to be obtained after bolus intravenous administration of the drug, we used a value from in the literature, i.e. $2.52 \mathrm{~h}^{-1}$ [22]. In vivo absorption appeared to be continuous, although its rate decreased strongly after 4 hours and became marginal after 12 hours. After 4-6 hours, the drug-release rate might well not be able to compensate for the fast elimination of acetaminophen, which could explain the decrease in drug plasma concentration for the SR curve in Figure 3.3. The high variations observed in Figure 3.4 might be explained, among others, by the same factors.

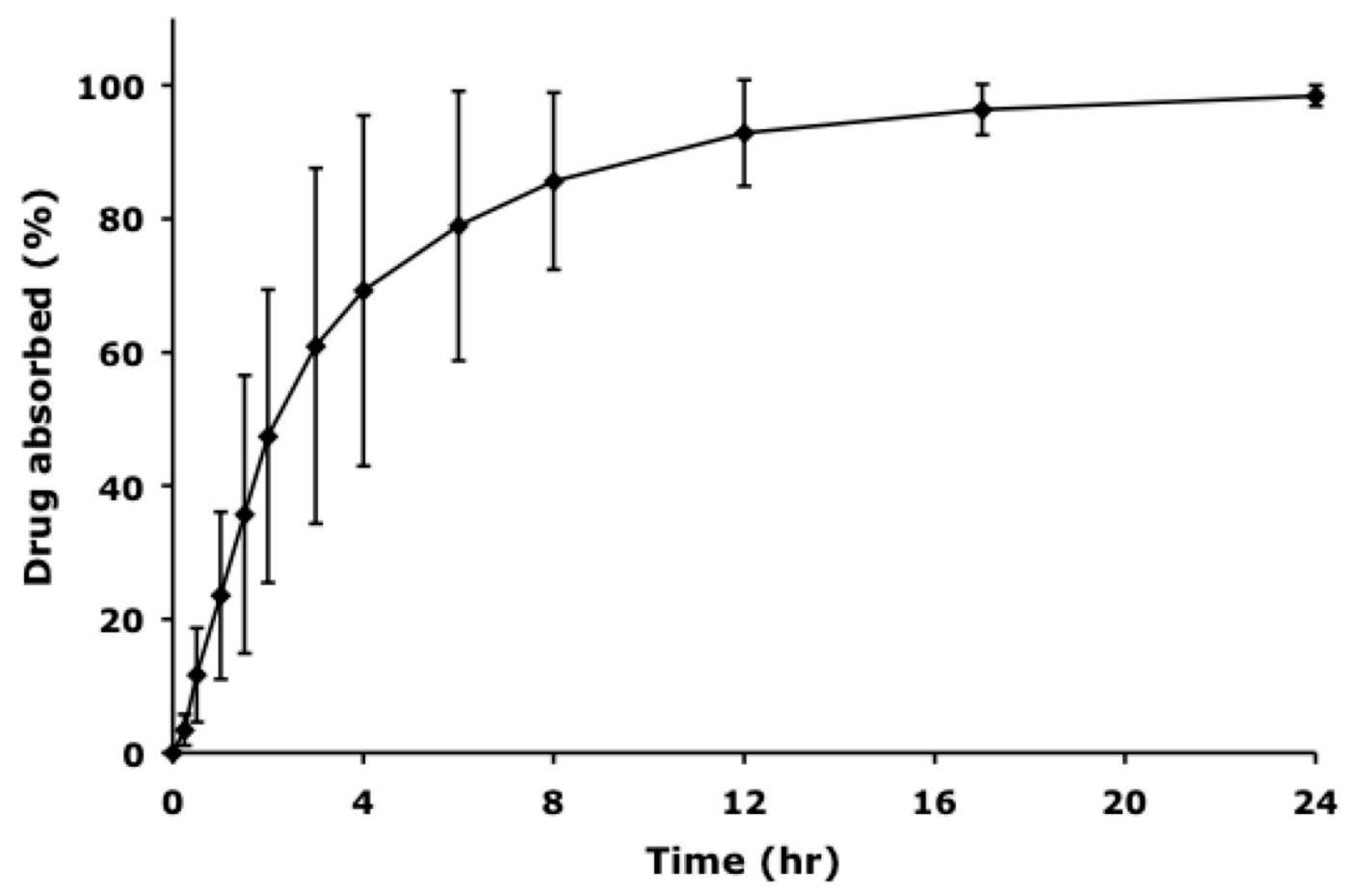

Figure 3.4 Cumulative percentage (mean $\pm \mathrm{SD}$ ) of acetaminophen absorbed following the oral administration of HASCA SR matrix tablets to 5 healthy volunteers. 
As suggested by the in vitro release profiles, the results of the in vivo study indicate that the HASCA formulation slowed acetaminophen absorption compared to the IR formulation, with significant drug-release for up to 12 hours. However, therapeutic blood levels were not maintained over a more extended time period due to the high $k$ of acetaminophen. Other limitations of this in vivo study include the small number of volunteers and, as mentioned above, the high inter-subject anatomic variability. However, our pilot study has clearly demonstrated the difference between the IR tablet and the HASCA SR tablet with respect to their release mechanisms. Further, the absence of immediate and fast disintegration of the HASCA tablets as well as their resistance to enzymatic degradation prove the potential of this new excipient as an efficient drug-delivery system. However, it must be remembered that ours was a non-blinded study where volunteers were not randomized, as the protocol was specific to each drug formulation.

\subsubsection{IVIVC}

To assess the relationship between in vivo acetaminophen absorption and in vitro acetaminophen release from the HASCA matrix tablet formulation, percent drug-release in vitro was compared to percent in vivo absorption at given time points. Figure 3.5 presents the results for the 5 volunteers and the average mean. Certain deviations from the ideal 1:1 relationship, which is represented by the straight line with slope $=1$ [23], were observed. While a linear relationship between the percentage released in vitro and the average percentage absorbed in vivo seems to be present for a portion of the curve, it appears that the release rate is faster in vivo. At this stage, we do not know if this is due to really faster release in vivo or to the fast elimination of acetaminophen, which renders application of the WagnerNelson method difficult and lacking in precision. Moreover, the intercept value should approximate zero for a "perfect" IVIVC, i.e. when the percentage absorbed varies in direct proportion to the percentage released in vitro. It is possible that in vivo, the matrix system takes a little more time to be hydrated enough to trigger burst-release. Finally, the variations seen from volunteer to volunteer are large, and volunteers seem to divide into 2 groups, but with such a small number of subjects, it is impossible to be sure. It is noteworthy that a poor 
IVIVC has already been reported for a SR matrix tablet containing similar doses of acetaminophen, although these results look more erratic and their dosage forms are more subject to $\mathrm{pH}$ variations than the present ones [23].

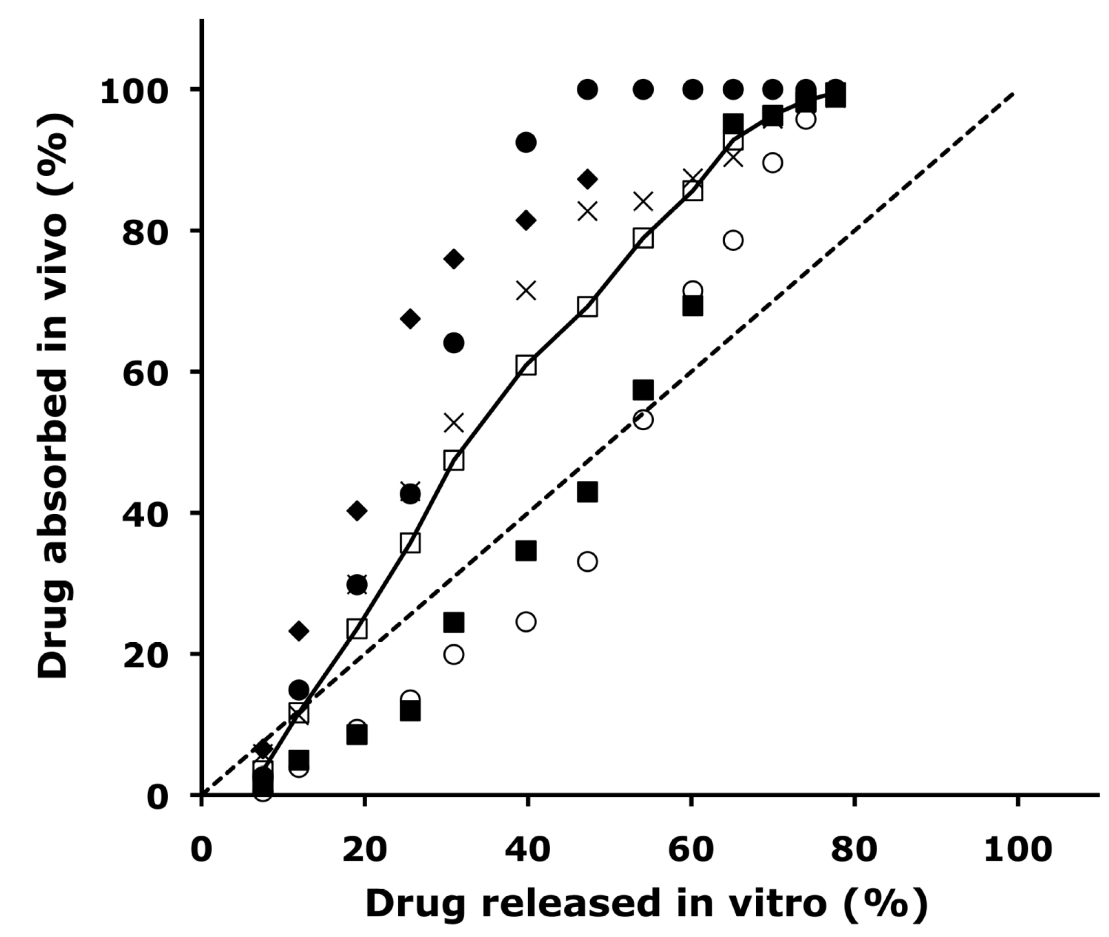

Figure 3.5 IVIVC plot for HASCA SR matrix tablets administered to 5 healthy volunteers volunteer $1, \mathrm{O}$ : volunteer $2, \mathrm{x}$ : volunteer 3 , @: volunteer 4 , $\mathbf{\square}$ : volunteer 5 , average, - - - -: linear).

However, it must be reiterated that the drug and the strength chosen, i.e. acetaminophen and $240 \mathrm{mg}$, were not the most appropriate for evaluating HASCA SR matrix tablets, since the drug's short half-life would require excessively large amounts of drug in each dosage unit to maintain sustained effects, forcing the dosage form itself to become unreasonably large. In addition, the elimination rate of this drug from the body was too high compared to the release rate from the proposed formulation, not allowing therapeutic blood levels to be maintained over an extended time period. Again, it must be emphasized that, as this was the first time that the new drug-delivery system was administered to human 
volunteers, their safety was the main concern when choosing the drug and its strength. Therefore, further investigations like increasing dosage strength and the release rate, are required to obtain a meaningful IVIVC for acetaminophen associated with this formulation.

\subsection{Conclusion}

HASCA is an interesting excipient for sustained drug-release in solid oral dosage forms. In addition to the easy manufacturing of tablets by direct compression, the results show that in vitro drug-release from an optimized HASCA formulation is not affected by either acidic $\mathrm{pH}$ value or acidic medium residence time. In addition, a compressed blend of HASCA with an optimized quantity of $\mathrm{NaCl}$ provides improved integrity to a pharmaceutical SR tablet for oral administration. The in vivo study demonstrates extended drug absorption, showing that the matrix tablets do not disintegrate immediately and resisted enzymatic attack well. Nevertheless, acetaminophen does not seem to be the most appropriate drug for this type of formulation.

\subsection{Acknowledgements}

Teresa D. Nabais gratefully acknowledges the scholarship support of Fundação para a Ciência e Tecnologia (Portugal). The help of Prof. M. Lesne is gratefully acknowledged.

\subsection{References}

[1] Bilariedis C.G., The structure and interactions of starch with food constituents, Can. J. Physiol. Pharmacol., 69, 60-78 (1991)

[2] Cartilier L., Moussa I., Chebli C. and Buczkowski S., Substituted Amylose as a Matrix for Sustained Drug Release, U.S. Patent No. 5,879,707 
[3] Chebli C., Moussa I., Buczkowski S. and Cartilier L., Substituted amylose as a matrix for sustained drug release, Pharm. Res., $\underline{16}(9), 1436-1440$ (1999)

[4] Chebli C. and Cartilier L., Effect of some physical parameters on the sustained-drug release properties of substituted amylose matrices, Int. J. Pharm., 193(02), 167-173 (2000)

[5] Cartilier L., Chebli C., Wang H.W., Moghadam S. and El-Leithy E., Substituted amylose matrices for oral drug delivery, Books of Abstracts of the ISAB $^{2}-20053^{\text {rd }}$ International Symposium on Advanced Biomaterials/Biomechanics, p 102, April 3-6, 2005, Montreal, Canada

[6] Chebli C., Cartilier L. and Hartman N., Substituted amylose as a matrix for sustained-drug release: a biodegradation study, Int. J. Pharm., 222(2), 183-189 (2001)

[7] Ungur M., Yonis N., Chebli C. and Cartilier L., The evaluation of carboxymethylamylose for oral drug delivery systems: from laboratory to pilot scale, Books of Abstracts of the ISAB $^{2}-20053^{\text {rd }}$ International Symposium on Advanced Biomaterials/Biomechanics, p 271, April 3-6, 2005, Montreal, Canada

[8] Cartilier L., Ungur M. and Chebli C., Tablet Formulation for Sustained Drug-release, Canadian Patent Application No. 2,491,665, December 24, 2004, PCT Application PCT/CA2005/001934, December 20, 2005

[9] Qureshi S., Tablet testing, in: Swarbrick J. (Ed.), Encyclopedia of Pharmaceutical Technology, 2004 Update Supplement, $2^{\text {nd }}$ edition, Marcel Dekker, New York, U.S.A., 2004, pp 481-490

[10] Food and Drug Administration, Extended Release Oral Dosage Forms: development, evaluation and application of in vitro-in vivo correlations, Center for Drug Evaluation and Research, Maryland, 1-24 (1997)

[11] Wagner J.G. and Nelson E., Kinetic analysis of blood levels and urinary excretion in the absorptive phase after single doses of drug, J. Pharm. Sci., 53(11), 1392-1403 (1964)

[12] Huang Y.B., Tsai Y.H., Yang W.C., Chang J.S., Wu P.C. and Takayama K., Once-daily extended-release tablet dosage form: formulation design and in vitro/in vivo investigation, Eur. J. Pharm. Biopharm., 58(3), 607-614 (2004)

[13] Forrest J.A., Clements J.A. and Prescott L.F., Clinical pharmacokinetics of paracetamol, Clin. Pharmacokinet., $\underline{7}(2)$, 93-107 (1982) 
[14] Gramatte T. and Richter K., Paracetamol absorption from different sites in the human small intestine, Br. J. Clin. Pharmacol., 37(6), 608-611 (1994)

[15] Yu L.X., Amidon G.L., Polli J.E., Zhao H., Mehta M., Conner D.P., Shah V.P., Lesko L.J., Chen M.L., Lee V.H.L. and Hussain A.S., Biopharmaceutics Classification System: the scientific basis for biowaiver extension, Pharm. Res., 19(7), 921-925 (2002)

[16] Cao Q.R., Choi Y.W., Cui J.H. and Lee B.J., Formulation, release characteristics and bioavailability of novel monolithic hydroxypropylmethylcellulose matrix tablets containing acetaminophen, J. Control. Release, 108(2-3), 351-361 (2005)

[17] Mattok G.L. and McGilveray I.J., The effect of food intake and sleep on the absorption of acetaminophen, Rev. Can. Biol., 32 (Suppl.), 77-84 (1973)

[18] Kawashima Y., Takeuchi H., Hino T., Niwa T., Lin T.L., Sekigawa F. and Ohya M., Preparation of prolonged-release matrix tablet of acetaminophen with pulverized lowsubstituted hydroxypropylcellulose via wet granulation, Int. J. Pharm., 99(2-3), 229-238 (1993)

[19] Hernandez J.I., Ghaly E.S., Malave A. and Marti A., Controlled-release matrix of acetaminophen-ethylcellulose solid dispersion, Drug Dev. Ind. Pharm.,_20(7), 1253-1265 (1994)

[20] Dalton J.T., Straughn A.B., Dickason D.A. and Grandolfi G.P., Predictive ability of level A in vitro-in vivo correlation for ringcap controlled-release acetaminophen tablets, Pharm. Res., 18(12), 1729-1734 (2001)

[21] Mayersohn M., Principles of drug absorption, in: Banker G. and Rhodes C. (Eds.), Modern Pharmaceutics, $4^{\text {th }}$ Edition, Marcel Dekker, New York, U.S.A., 2002, pp 23-66

[22] Perucca E. and Richens A., Paracetamol disposition in normal subjects and in patients treated with antiepileptic drugs, Br. J. Clin. Pharmacol., 7(2), 201-206 (1979)

[23] Parojcic J., Duric Z., Jovanovic M., Ibric S. and Jovanovic D., Influence of dissolution media composition on drug release and in-vitro/in-vivo correlation for paracetamol matrix tablets prepared with novel carbomer polymers, J. Pharm. Pharmacol., 56 (6), 735-741 (2004) 


\section{Chapter 4. High-amylose carboxymethyl starch matrices: development and characterization of tramadol hydrochloride sustained-release tablets for oral administration}

Teresa Nabais ${ }^{\mathrm{a}}$ and Grégoire Leclair ${ }^{\mathrm{a}, *}$

${ }^{a}$ Faculty of Pharmacy, University of Montreal, Montreal (Quebec) Canada;

* Corresponding author: Faculty of Pharmacy, University of Montreal, Montreal (Quebec) Canada

Keywords: Drug delivery; sustained-release; excipient; polymer; tablet; matrix; starch; amylose; in vitro; once-daily; twice-daily 


\subsection{Presentation of the article and contribution of the authors}

This article presents a formulation screening carried out in order to select once-daily and twice-daily SD HASCA matrix tablets with two common dosages of tramadol hydrochloride (100 $\mathrm{mg}$ and $200 \mathrm{mg}$ ). The results regarding dissolution studies in a $40 \%$ ethanol medium are also included. This article was submitted to ISRN Pharmaceutics on January 22, 2014.

The contribution of each author to the publication was:

Teresa Nabais - Execution of all dissolution testing; analysis of data resulting from dissolution testing; writing of the article.

Grégoire Leclair - Project coordinator and director at the time of the publication; revision of the article.

\subsection{Abstract}

Substituted amylose (SA) polymers were produced from high-amylose corn starch by etherification of its hydroxyl groups with chloroacetate. Amorphous high-amylose sodium carboxymethyl starch (HASCA), the resulting SA polymer, was spray dried to obtain an excipient (SD HASCA) with the better binding and sustained-release (SR) properties. Tablets containing different percentages of SD HASCA and tramadol hydrochloride were produced by direct compression and evaluated for dissolution. Once-daily and twice-daily SD HASCA tablets containing two common dosages of tramadol hydrochloride (100 mg and $200 \mathrm{mg}$ ), a freely water-soluble drug, were successfully developed. These SR formulations presented high crushing forces, which facilitate further tablet processing and handling. When exposed to both a $\mathrm{pH}$ gradient simulating the $\mathrm{pH}$ variations through the gastrointestinal tract and to a $40 \%$ ethanol medium, a very rigid gel formed progressively at the surface of the tablets providing controlled drug-release properties. These properties indicated that SD HASCA was a promising and robust excipient for oral, sustained drug-release, which may possibly minimize 
the likelihood of dose dumping and consequent adverse effects, even in the case of coadministration with alcohol.

\subsection{Introduction}

Starch is a naturally occurring and a biodegradable polymer that is metabolized by the human body. Besides being non-toxic, starch is an abundant, cost-effective and renewable material [1]. Due to these advantages, starches and modified starches have been widely and safely used in the food industry as thickeners, enhancers of organoleptic properties or texture modifiers, and in the pharmaceutical industry as fillers, binders, disintegrants [2] and, more recently, as hydrophilic excipients for sustained drug-release. Numerous starch-modification methods, such as chemical [3], physical (i.e. gelatinization) [4], enzymatic [5], or a combination thereof, have been employed to produce new starch products with specific properties. Starch is a good candidate for chemical reaction or transformation because it is composed of amylose and amylopectin, two glucose polymers presenting three hydroxyl groups accessible as chemically active functional entities. Some of the modifications commonly employed to prepare starch derivatives are carboxymethylation, ethoxylation and oxidation [6].

Substituted amylose (SA) has been introduced as a promising pharmaceutical excipient for sustained drug-release. SA matrix tablets have been prepared by direct compression, which is the easiest way to manufacture an oral dosage form and consists of dry mixing of drug and SA polymers followed by compression [7,8]. The first SA polymer showing good properties as an excipient for sustained-release (SR) was produced by an etherification process using high-amylose corn starch and glycidol as the substituent, and was referred to as SA,G-2.7, where $G$ defines glycidol and 2.7 represents the degree of substitution (DS), expressed as the ratio of mole of substituent per kilogram of amylose [7,8]. These properties included almost constant in vitro drug-release [7-9], no significant influence of compression forces (CF) ranging from 0.5 to 5.0 tons $/ \mathrm{cm}^{2}$ on the drug-release properties $[9,10]$, large ranges of use for drug-loading, drug-solubility, and tablet weight (TW) [7,8], and unusually high crushing force values [10]. 
The use of sodium chloroacetate or chloroacetic acid in place of nonionic substituents like glycidol has been subsequently proposed to produce an excipient more readily acceptable by regulatory agencies for its non-toxicity $[11,12]$. Sodium carboxymethyl starch with low amylose content was already a well-known disintegrant agent in immediate-release (IR) tablets [13]. High-amylose sodium carboxymethyl starch (HASCA, where "HAS" means highamylose substituted starch and "CA" defines the substituent used, herein chloroacetate), contrariwise, has been introduced as a promising pharmaceutical excipient for oral SR tablets prepared by direct compression $[11,12]$. Contramid ${ }^{\circledR}$, a cross-linked high amylose starch, has already been used in tablets as a retarding agent [14].

After being initially produced on laboratory scale, the polymer was obtained on an industrial pilot scale. However, these latter batches of polymer were unsuitable for tableting and sustained drug-release due to inadequate physical properties. For this reason, the material was spray dried (SD) to obtain a compressible excipient. This procedure, which has been described elsewhere [6], resulted in SD HASCA, the polymer used in the current study.

Studies on the in vitro release characteristics of acetaminophen from tablets composed of SD HASCA, drug and sodium chloride have demonstrated the suitability of this polymer as a sustained drug-release excipient. No burst-effect was observed, even in the presence of large amounts of soluble sodium chloride in the formulation [15-17]. Moreover, the release of acetaminophen from optimized SD HASCA formulations is not significantly influenced by the variations of $\mathrm{pH}$ and residence time in acidic media [17]. These prior results suggest that drugrelease from SD HASCA tablets may not be affected by intra- and inter-subject variations in gastric $\mathrm{pH}$ or gastric residence time. Acetaminophen (phenol, $p K_{a}$ 9.51) and tramadol hydrochloride (tertiary amine, $p K_{a} 9.41$ ) are completely non-ionized and completely ionized at physiological $\mathrm{pH}$, respectively. Moreover, a first in vivo study where the same optimized SD HASCA formulation was administered orally to healthy human subjects has demonstrated extended acetaminophen plasma levels, and that the gel formed by the polymer was strong enough to resist both the powerful peristaltic contractions normally occurring in the fasted state and biodegradation in the intestine [17]. Other features of SD HASCA that confirm the potential of this polymer for sustained drug-release include its excellent binding properties in the absence of binders, which resulted in very high crushing forces $[6,15,16]$. A strong 
dependence of drug-release on TW has also been found, corresponding a TW rise to an increased total release time [16].

Tramadol is a synthetic, centrally acting analgesic agent with a dual mechanism of action [18]. It has proven to be effective in the treatment of moderate to moderately severe pain without causing severe side effects and organ damages [19]. For the treatment of chronic pain, frequent oral administration of IR tramadol is required (at least four times daily) because of its mean elimination half-life, which can vary from 5 to $7 \mathrm{~h}$ in humans [20]. In adults and adolescents, the usual oral dosage regimen is 50 to $100 \mathrm{mg}$ every 4 to 6 hours, with a maximum dosage of $400 \mathrm{mg} /$ day [18]. Consequently, oral formulations of tramadol that provide a more gradual release of the drug have been developed with the aim of reducing the frequency of administration, minimizing acute adverse effects, and thus improving the patient compliance $[21,22]$. After oral administration, tramadol is rapidly and almost completely absorbed. Its bioavailability of $70 \%$ after a single administration can be attributed entirely to extensive first pass metabolism [23]. However, at steady state, the bioavailability of a SR formulation has been shown to be $100 \%$ relative to an IR formulation [24], which represents another advantage of SR formulations of tramadol. Bioequivalence after dose adjustment between IR and SR formulations has been demonstrated [20]. Tramadol hydrochloride is readily soluble in water and ethanol and hence judicious selection of release retarding excipients is necessary to achieve an extended in vivo input rate of the drug.

SR formulations, especially those designed for once-daily administration, contain amounts of drug that can be potentially risky if released as a burst, commonly called dose dumping. Tramadol hydrochloride may cause severe side effects if high plasma concentrations are reached quickly. Inadvertent or intentional misuse of a SR formulation, with consequent dose dumping, may include breaking, crushing tablets and simultaneous co-ingestion of the medication with high doses of ethanol. Therefore, the robustness of SD HASCA tablets in an ethanolic medium as well as their crushing strength values are important factors to take into account.

In this study, the vitro release characteristics from matrix tablets comprising tramadol hydrochloride as the model drug and SD HASCA as the only SR excipient were evaluated in a series of buffers simulating the $\mathrm{pH}$ of the fluids throughout the gastrointestinal tract. Tablets intended to twice-daily and once-daily administration with $100 \mathrm{mg}$ and $200 \mathrm{mg}$ of drug were 
developed and the dissolution profiles of these tablets were compared with those of marketed tramadol hydrochloride SR formulations of same drug content. The resistance of the gel formed by SD HASCA to an alcoholic medium was also investigated.

\subsection{Materials and methods}

\subsubsection{Materials}

Amorphous HASCA (Eurylon 6, batch 3910, ref. 799511) was provided by Roquette Frères (Lestrem, France) and contained approximately $60 \%$ of amylose and $40 \%$ of amylopectin. The DS was equal to 0.045 (number of moles of substituent/number of moles of anhydroglucose) [16]. Tramadol hydrochloride was purchased from Jubilant Organosys Ltd. (Nanjangud, Mysore, India). Commercially available tramadol tablets Tridural ${ }^{\circledR} 100 \mathrm{mg}$ and $200 \mathrm{mg}$, Ralivia ${ }^{\circledR} 200 \mathrm{mg}$, Zytram $^{\circledR}$ XL $200 \mathrm{mg}$, Topalgic ${ }^{\circledR}$ LP $100 \mathrm{mg}$ and $200 \mathrm{mg}$ as well as Contramal $^{\circledR}$ LP $200 \mathrm{mg}$ were purchased from pharmacies in Canada and in France. All chemicals were of reagent grade and were used without further purification.

\subsubsection{Preparation of HASCA tablets}

Amorphous HASCA, as provided by its manufacturer, did not possess the required binding and SR properties to be used as a suitable material for SR tablets prepared by direct compression. Therefore, this polymer was spray-dried as described by Brouillet et al. [6] by first preparing a suspension of HASCA in a mixture of water and ethanol (final water/ethanol ratio of 1.33, $4 \mathrm{~mL}$ of ethanol/g of HASCA), and then spray drying using a Büchi B-290 Mini Spray-Dryer (Flawill, Switzerland).

Tablets were prepared by direct compression using a 30-ton manual hydraulic press (referred herein as HP, C-30 Research \& Industrial Instruments Company, London, U.K.) equipped with round flat-faced tooling (diameter $12.6 \mathrm{~mm}$ ) or a single-stroke press machine (referred herein as SSP, Manesty F3 Machine, Manesty Machines Ltd., Liverpool, UK) also 
equipped with round flat-faced tooling (diameter $11.1 \mathrm{~mm}$ ). In the HP, compression force was adjusted to target a specific $\mathrm{CF}$, and in the SSP, compression force was adjusted to target specific thickness. SD HASCA tablets of different total weights containing different percentages of polymer and drug were produced as described in Table 4.1.

Table 4.1 SD HASCA tablets formulation, press used, target CF and thickness and selected twice-daily and once-daily tablets.

\begin{tabular}{|c|c|c|c|c|c|}
\hline $\begin{array}{l}\text { TW } \\
(\mathrm{mg})\end{array}$ & $\%$ of drug $(w / w)$ & $\begin{array}{l}\text { Tablet } \\
\text { press }\end{array}$ & $\begin{array}{l}\text { Target CF } \\
\left(\text { tons } / \mathrm{cm}^{2}\right), \mathrm{HP}\end{array}$ & $\begin{array}{l}\text { Target thickness } \\
(\mathrm{mm}), \text { SSP }\end{array}$ & $\begin{array}{l}\text { Selected twice- } \\
\text { daily and once- } \\
\text { daily tablets }\end{array}$ \\
\hline \multirow[t]{5}{*}{400} & 12.5 & HP & 2.5 & - & - \\
\hline & 25 & HP & 2.5 & - & - \\
\hline & 37.5 & HP & 2.5 & - & - \\
\hline & 50 & HP & 2.5 & - & - \\
\hline & 60 & HP & 2.5 & - & - \\
\hline 450 & 22.22 & $\mathrm{HP}$ & 2.5 & - & $\begin{array}{l}\text { Twice-daily (not } \\
\text { selected) }\end{array}$ \\
\hline \multirow[t]{5}{*}{500} & 20 & HP & 2.5 & - & - \\
\hline & 30 & HP & 2.5 & - & - \\
\hline & 40 & HP & 2.5 & - & $\begin{array}{l}\text { Twice-daily (not } \\
\text { selected) }\end{array}$ \\
\hline & 50 & HP & 2.5 & - & - \\
\hline & 60 & HP & 2.5 & - & - \\
\hline \multirow[t]{4}{*}{700} & 14.3 & HP & 1 & - & - \\
\hline & 14.3 & HP & 1.5 & - & - \\
\hline & 14.3 & HP & 2 & - & - \\
\hline & 14.3 & HP & 2.5 & - & $\begin{array}{l}\text { Once-daily (not } \\
\text { selected) }\end{array}$ \\
\hline \multirow[t]{5}{*}{800} & 12.5 & HP & 2.5 & - & - \\
\hline & 25 & HP & 2.5 & - & $\begin{array}{l}\text { Once-daily (not } \\
\text { selected) }\end{array}$ \\
\hline & 37.5 & HP & 2.5 & - & - \\
\hline & 50 & HP & 2.5 & - & - \\
\hline & 60 & HP & 2.5 & - & - \\
\hline 450 & $22.22(100 \mathrm{mg})$ & SSP & - & 4.63 & $\begin{array}{l}\text { Twice-daily } \\
\text { (Selected) }\end{array}$ \\
\hline 500 & $40(200 \mathrm{mg})$ & SSP & - & 5.15 & $\begin{array}{l}\text { Twice-daily } \\
\text { (Selected) }\end{array}$ \\
\hline 700 & $14.3(100 \mathrm{mg})$ & SSP & - & 6.98 & $\begin{array}{l}\text { Once-daily } \\
\text { (Selected) }\end{array}$ \\
\hline 800 & $25(200 \mathrm{mg})$ & SSP & - & 7.43 & $\begin{array}{l}\text { Once-daily } \\
\text { (Selected) }\end{array}$ \\
\hline
\end{tabular}




\subsubsection{Tablets characterization and in vitro drug-release evaluation}

The crushing forces (Newton) of SD HASCA tablets were measured using a hardness tester (Pharma Test Type PTB 301, Hainburg, Germany) and their thickness using an electronic caliper (Starrett, 799A Series).

The in vitro drug-release properties of SD HASCA SR tablets in simulated gastric fluid (stage I) and simulated intestinal fluids (stage II and stage III) were assessed using a USP apparatus $2\left(900 \mathrm{~mL}, 37^{\circ} \mathrm{C}, 50 \mathrm{rpm}\right)$ [25]. During stage I, the tablets were exposed to hydrochloric acid buffer (pH 1.2), simulating gastric $\mathrm{pH}$ (SGF) for $1 \mathrm{~h}$. During stage II, the tablets were exposed to phosphate buffer ( $\mathrm{pH}$ 6.8) simulating jejunum $\mathrm{pH}$ (SIF I) for an additional 3 h. Finally, the tablets were exposed to a second phosphate buffer ( $\mathrm{pH} 7.4$ ) simulating ileum pH (SIF II) until the end of the assay (total time of $24 \mathrm{~h}$ ). All standard buffer solutions were prepared according to the USP.

In addition, dissolutions tests in a $40 \%$ ethanol in water solution were performed to assess the release properties of a SD HASCA formulation and a commercial formulation with the same drug content and the same frequency of administration. This percentage of ethanol was chosen because it represents a standard strength of alcoholic spirits [26]. Tests in alcohol were carried out during $7 \mathrm{~h}$.

To avoid eventual floating to the surface of the medium or adhesion to the vessel, each tablet was placed inside a perforated cylindrical plastic cage (Fig. 4.1). The cage had enough internal space for drug-release to occur from all sides of the matrix and its perforations were big enough not to interfere with the circulation of the medium inside the dissolution vessel but, on the other hand, were small enough to keep the tablets inside. 


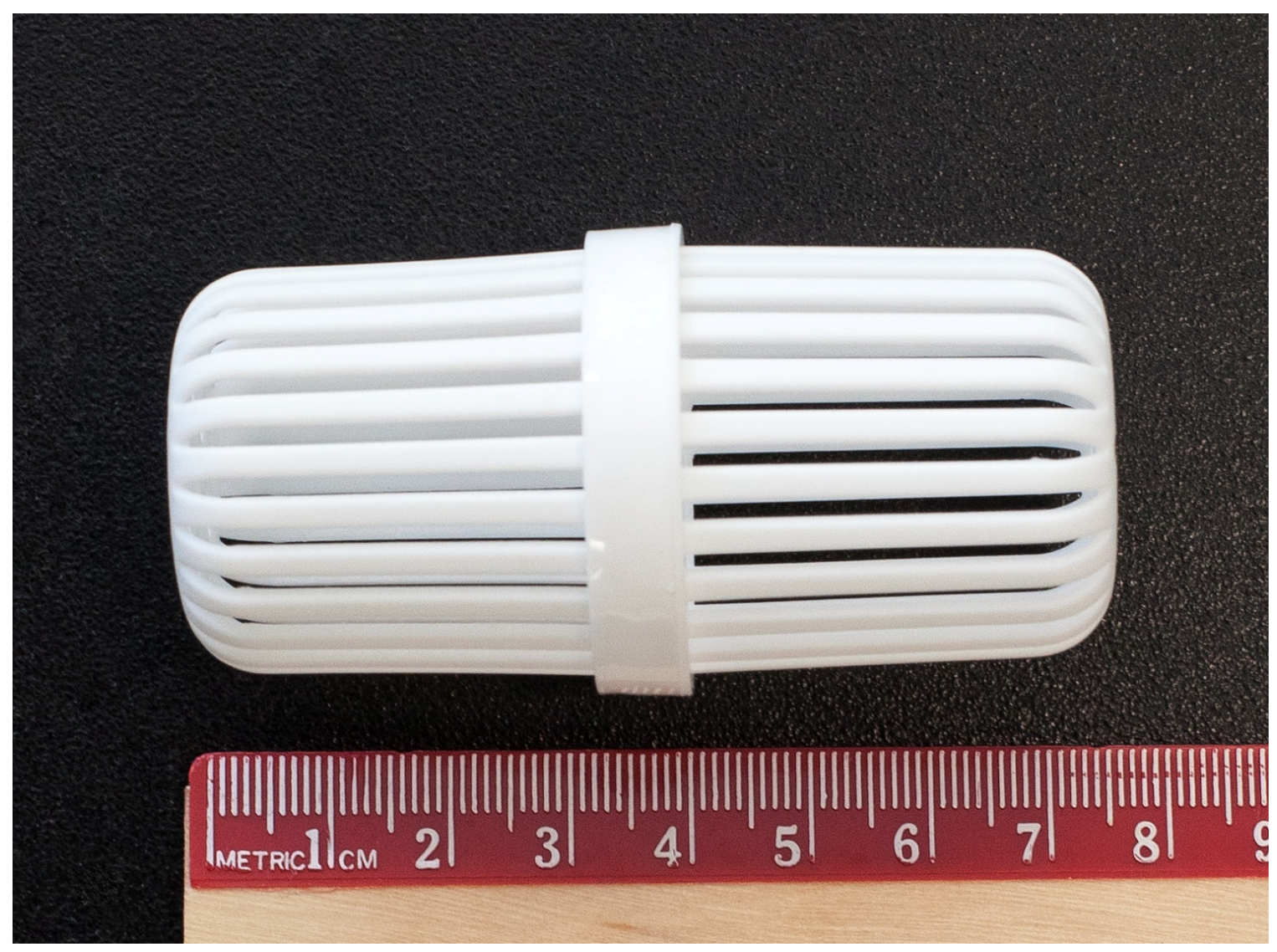

Figure 4.1 Plastic cage used in the dissolution experiments to avoid eventual adhesion to the vessel or floating.

Due to the presence of the cages at the bottom of the dissolution vessel, the paddle relative position in the vessel was positioned $5 \mathrm{~cm}$ above what it is settled by USP. After each sampling, an equivalent volume of fresh medium $(10 \mathrm{~mL})$ was added. Each experiment was performed in triplicate. The occurrence of macroscopic transformations in the matrix tablets and their eventual influence on the release profiles was registered. Samples were filtered through syringe filters $(0.45 \mathrm{~mm}$, nylon). Tramadol hydrochloride release was followed by UV spectrometry $(271 \mathrm{~nm})$. A calibration curve was prepared for each of the three mediums $(\mathrm{pH}$ 1.2, 6.8 and 7.4) as well as for the ethanolic medium, and before the measurement of the samples resulting from each dissolution test. The drug-release results are expressed as cumulative percentage $(\%)$ as a function of time $(\mathrm{h})$. 
The in vitro release properties of twice-daily and once-daily commercial formulations with $100 \mathrm{mg}$ or $200 \mathrm{mg}$ of tramadol hydrochloride were also evaluated using the same experimental conditions, so as to provide a direction regarding the desired release times. The twice-daily dosage forms tested were Topalgic ${ }^{\circledR}$ LP $100 \mathrm{mg}$, Topalgic ${ }^{\circledR}$ LP $200 \mathrm{mg}$ and Contramal $^{\circledR}$ LP $200 \mathrm{mg}$, and the once-daily dosage forms tested were Tridural ${ }^{\circledR} 100 \mathrm{mg}$, Tridural $^{\circledR} 200 \mathrm{mg}$, Ralivia ${ }^{\circledR} 200 \mathrm{mg}$ and Zytram $^{\circledR}$ XL $200 \mathrm{mg}$.

\subsubsection{Modifications to the United States Pharmacopeia (USP) method}

In the first in vitro studies on the release of tramadol hydrochloride from SD HASCA matrix tablets some formulations showed a propensity to float or to adhere to the bottom of the vessel. This propensity was higher as the concentration of tramadol hydrochloride increased. This situation makes the tablets very susceptible to large variations in hydrodynamic conditions that prevail in a dissolution vessel. In addition, the tablets floating at the surface of the dissolution medium or adhering to the glass surface had a lower surface area in contact with the medium. This situation can affect drug-release from the tablets, as the exposed surface area is a major factor determining release kinetics both in erosion-controlled as well as diffusion-controlled systems [27].

The use of perforated cylindrical plastic cages placed below the paddles in the dissolution vessels showed to be successful in preventing adhesion to the vessel and floating. This solution was chosen in detriment of the use of USP apparatus I (basket) as the use of this apparatus can interfere with the free swelling of the matrix. However, the paddle relative position had to be at a higher height compared to what is settled by the USP because of the size of the cage. Hence, the influence of the paddle position on drug-release was evaluated. The cumulative percentage of acetaminophen released in vitro from 700-mg matrix tablets comprising $40 \%$ of acetaminophen and $60 \%$ of HPMC when the paddle relative position in the dissolution vessel was as settled by USP or placed about $5 \mathrm{~cm}$ above what it is settled by USP was equivalent ( $f_{2}$ equal to 99.77$)$. 


\subsubsection{Statistical analysis and formulations comparison}

The arithmetic average and the standard deviation of the cumulative \% of drug released at predetermined time intervals were calculated.

Dissolution profiles were compared using the similarity factor $\left(f_{2}\right)$, according to the following equation proposed by Moore and Flanner [28]:

$$
f_{2}=50 \times \log \left\{\left[1+(1 / n) \sum_{t=1}^{n}\left(R_{t}-T_{t}\right)^{2}\right]^{-0.5} \times 100\right\}
$$

where $R_{t}$ and $T_{t}$ are the percent dissolved of the reference and test products at each time point $t$ and $n$ is the number of pool points.

Two dissolution profiles are similar if $f_{2}$ is between 50 and 100 according to the Food and Drug Administration (FDA) and the European Agency for the Evaluation of Medical Products (EMEA). However, the closer to $100(\%) f_{2}$, the more similar the dissolution profiles are. The dissolution profiles were also compared using the values of the time to release $50 \%$ (T50\%) and to release $90 \%$ of drug (T90\%).

\subsection{Results and discussion}

\subsubsection{Measurement of tablet crushing strengths in order to guarantee the reproducibility of the spray drying method}

The reproducibility of the method used to transform amorphous HASCA into SD HASCA was assessed through the evaluation of tablet crushing forces for different batches. SD HASCA tablets weighting $200 \mathrm{mg}$ were prepared by direct compression. The excipient was compressed at a CF of 2.5 tons $/ \mathrm{cm}^{2}$ using a HP using a dwell time of 20 seconds. The values of the crushing forces of 200-mg tablets produced from different batches of SD HASCA varied between $124.9 \pm 10.5$ and $148.5 \pm 3.2$ Newton (arithmetic averages \pm standard 
deviations). Therefore, the crushing forces were considered reproducible. As non-SD HASCA particles did not possess any binding properties and the SD procedure resulted in a decrease in particle size of the polymer, it has been hypothesized that these unusual high crushing strength values are due, in part, to the particle size reduction, which corresponds to a higher surface area of the particulate product and provides a higher number of binding points. In addition, it is believed that the combination of water and ethanol in the SD process has a plasticizer effect, causing partial melting of the polymer and particle re-arrangement under compression [6].

\subsubsection{Formulation screening and development of twice-daily and once-daily SD HASCA formulations with $100 \mathrm{mg}$ and $200 \mathrm{mg}$ of tramadol hydrochloride}

The values of the T50\% and T90\% of tramadol hydrochloride from tablets of different total weights and containing different percentages of polymer and tramadol hydrochloride (compressed with the HP) are summarized in Table 4.2. Increasing the percentage of tramadol hydrochloride in the tablet led to an increase in the drug-release rate for all TWs, which is a common observation for hydrophilic matrices. In addition, the total release time increased significantly as a function of TW. The T50\% was, though, less affected by TW than the T90\%.

When immersed in the dissolution mediums, a very rigid gel layer with high mechanical strength in the swollen state formed on the surface of the tablets. This strong gel layer, which is formed by hydrogen bonding between the hydroxyl groups of the soluble gelled starch chains, has been documented before for SD HASCA $[15,16]$. In addition, its formation has been related to the $\mathrm{pH}$-independent $\mathrm{SR}$ behaviour of high-amylose sodium carboxymethyl starch produced at low DS, as it is the case of SD HASCA [29]. The gel layer formed by SD HASCA showed a lower degree of swelling when compared to the gel layer formed by hydroxypropylmethylcellulose (HPMC) (results not shown). The most important aspect of the release mechanism from tablets constituted by hydrophilic polymers is known to be the formation of a gel layer around the dry core of the matrix in response to water penetration. This gel layer hinders fast inward medium penetration and outward drug 
diffusion. Phenomena that govern gel layer formation and, therefore, drug-release, are penetration of the dissolution medium into the matrix, resulting in polymer hydration and swelling (relaxation process), drug dissolution and diffusion through the layer of swollen polymer, and erosion on the surface of the matrix. The formation of the gel layer is caused by the polymer-state transition. The gel layer acts like a barrier against the fast release of drugs whilst controlling at the same time the penetration of aqueous medium and the diffusion of drug.

Table 4.2 Time to release 50\% (T50\%) and 90\% (T90\%) of drug from SD HASCA tablets with total weights between $400 \mathrm{mg}$ and $800 \mathrm{mg}$, and \% of drug (w/w) between $12.5 \%$ and $60 \%$.

\begin{tabular}{|c|c|c|c|c|c|c|c|c|c|c|c|}
\hline \multirow[b]{2}{*}{$\begin{array}{l}\text { TW } \\
\text { (mg) }\end{array}$} & \multirow[b]{2}{*}[\mathrm{T}50\%,\mathrm{T}90\%]{$(\mathrm{h})$} & \multicolumn{10}{|c|}{$\%$ of drug $(w / w)$} \\
\hline & & 12.5 & 14.3 & 20 & 22.22 & 25 & 30 & 37.5 & 40 & 50 & 60 \\
\hline \multirow[t]{2}{*}{400} & $\mathrm{~T} 50 \%$ & 1.8 & - & - & - & 1.5 & - & 1.4 & - & 1.3 & 1.1 \\
\hline & $\mathrm{T} 90 \%$ & 6.5 & - & - & - & 5.3 & - & 4.5 & - & 4.6 & 3.5 \\
\hline \multirow[t]{2}{*}{450} & $\mathrm{~T} 50 \%$ & - & - & - & 1.9 & - & - & - & - & - & - \\
\hline & Т90\% & - & - & - & 6.4 & - & - & - & - & - & - \\
\hline \multirow[t]{2}{*}{500} & $\mathrm{~T} 50 \%$ & - & - & 2.55 & - & - & 2.05 & - & 1.9 & 1.75 & 1.4 \\
\hline & $\mathrm{T} 90 \%$ & - & - & 8.75 & - & - & 7.6 & - & 6.7 & 5.9 & 4.65 \\
\hline \multirow[t]{2}{*}{700} & $\mathrm{~T} 50 \%$ & - & 4 & - & - & - & - & - & - & - & - \\
\hline & $\mathrm{T} 90 \%$ & - & 14.4 & - & - & - & - & - & - & - & - \\
\hline \multirow[t]{2}{*}{800} & $\mathrm{~T} 50 \%$ & 4.9 & - & - & - & 4.1 & - & 3.3 & - & 2.7 & 2.5 \\
\hline & Т90\% & 18.4 & - & - & - & 16.4 & - & 11.5 & - & 10.25 & 9.3 \\
\hline
\end{tabular}


As the size of the internal dry and/or partially hydrated core of the tablets increases as a function of TW for the same concentration of drug, if the core is considered as a drug reservoir, the larger is the internal reservoir the longer is the time to empty it, which explains the increase of the total release time as a function of TW. Besides, as demonstrated in a previous study, augmenting TW heightens the contribution of diffusion to the detriment of erosion as the main mechanism controlling drug-release, leading to a decline in the drugrelease rate [16]. Furthermore, a combination of two release mechanisms has been described as controlling the kinetics of drug-release from SD HASCA matrices: drug diffusion through the gel layer after drug dissolution (Fickian diffusion) and polymer disentanglement and erosion (Case-II relaxational release) [16]. Fickian diffusion release occurs by diffusion of drug molecules due to a concentration gradient. Case-II relaxational release is the mechanism associated with polymer state-transition from the glassy, a solid state (dry polymer), to the rubbery state, an expanded and flexible state, which is linked with the polymer swelling process [30]. Similar conclusions were reported for the transport of acetaminophen from nonionic SA matrices [8,9]. It is known that drug concentration and thickness of the gel layer are the factors governing drug-release [31]. At lower drug concentrations, that is, higher polymer concentrations, diffusion is the main transport mechanism, and thus the total release time was longer. However, as drug concentration increases the drug-release rates also increase. Tramadol hydrochloride is freely soluble in water, and thus dissolves quickly in aqueous medium. Its high solubility along with an increase in drug loading resulted in higher drug concentration in the gel. In addition, the high hydrophilicity of tramadol hydrochloride promotes the penetration of the dissolution medium into the matrices. Above a certain drugloading threshold, the quantity of absorbed water may be such that the interactions waterpolymer become superior to the interactions polymer-polymer, causing chain disentanglement and polymer dissolution [32]. As a result, progressive erosion appears on the surface of the tablets, leading to a decrease in the thickness of the gel layer and an increase in the drugrelease rate. Indeed, it has been reported that the gel strength is an essential factor in the matrix performance and is controlled not only by the viscosity and chemical structure of the rubbery polymer but also by its concentration [31]. Therefore, lower SD HASCA concentrations in the tablet led to lower gel strengths, and thus to higher release rates, owing to higher erosion. Besides, it can be hypothesized that at certain drug/polymer ratio the release 
process will become governed mainly by erosion. However, opposite results have been observed for tablets containing SD HASCA, acetaminophen and sodium chloride. In this case, an increase in drug loading corresponded to an increase in total release time, until a certain level where erosion occurred [16]. It is important to take into consideration the higher solubility of tramadol hydrochloride compared to acetaminophen. Indeed, when formulating SD HASCA matrices, not only the concentration of the components has to be taken into account but also their solubility, nature and interactions between them.

As the values of the time to release $25 \%$ of drug (T25\%) for different TWs were similar, especially for the 400-mg tablets, these values are not presented. The T25\% corresponds usually to the burst effect and depends on the amount of drug on the table surface available for immediate dissolution and release in the medium.

The formulation screening allowed the investigation of the most adequate total weights for four formulations with SD HASCA as the SR excipient: two twice-daily with $100 \mathrm{mg}$ and $200 \mathrm{mg}$ of tramadol hydrochloride, and two once-daily with $100 \mathrm{mg}$ and $200 \mathrm{mg}$ of tramadol hydrochloride. In order to select these formulations, the in vitro drug-release profiles from tablets with $100 \mathrm{mg}$ and $200 \mathrm{mg}$ of drug, which were produced using the HP to study the influence of drug content and TW on the dissolution rate, for the formulation screening described above, were compared to the in vitro drug-release profiles of commercially available twice-daily and once-daily tramadol hydrochloride formulations with the same drug content. The release profile from the tablets with a total weight of $700 \mathrm{mg}$, containing $100 \mathrm{mg}$ of drug and compressed with the HP at 2.5 tons $/ \mathrm{cm}^{2}$ for 20 seconds, which were prepared to study the influence of the $\mathrm{CF}$ on the release rate, was also compared to the release profile of commercial formulations. Figure 4.2 shows the drug-release profiles from the commercial once-daily dosage forms. 


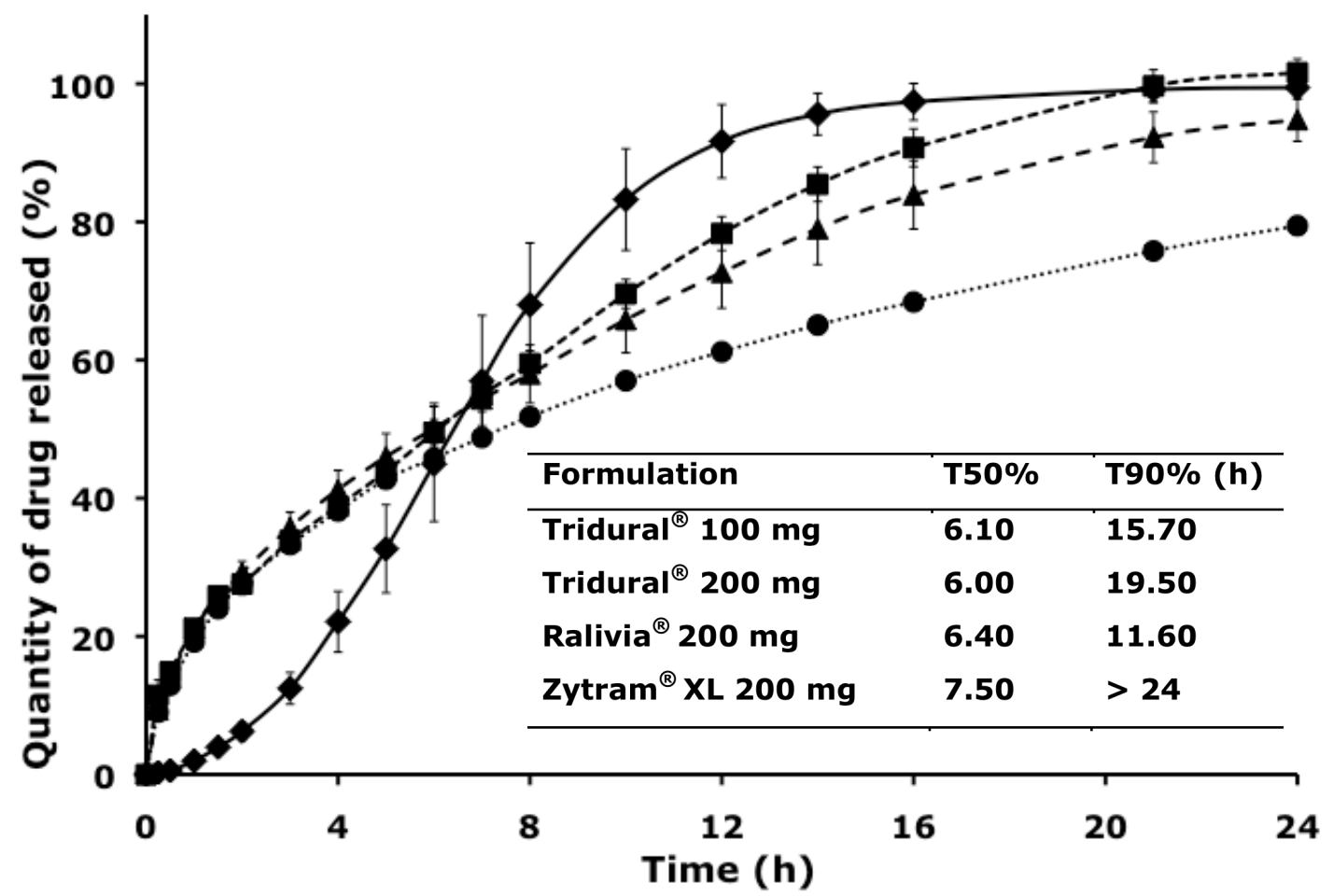

Figure 4.2 Cumulative \% of tramadol hydrochloride released in vitro from once-daily commercial formulations $\left(\boldsymbol{\square}\right.$ : Tridural ${ }^{\circledR} 100 \mathrm{mg}, \boldsymbol{\Delta}$ : Tridural $^{\circledR} 200 \mathrm{mg}, \diamond:$ Ralivia $^{\circledR} 200 \mathrm{mg}$, • : Zytram ${ }^{\circledR}$ XL $\left.200 \mathrm{mg}\right)$.

The values of $\mathrm{T} 50 \%$ and $\mathrm{T} 90 \%$ for the tablets weighting $500 \mathrm{mg}$ with $200 \mathrm{mg}$ of tramadol hydrochloride were 1.9 and 6.7 hours (Table 4.5), very similar to the values for Topalgic ${ }^{\circledR}$ LP $200 \mathrm{mg}, 2$ and 6.9 hours, respectively (Table 4.3). In addition, the $f_{2}$ resultant from the comparison of their profiles resulted in value of 86.54. Consequently, the 500-mg tablet with $200 \mathrm{mg}$ of drug was chosen as the twice-daily formulation with $200 \mathrm{mg}$ of drug.

An evaluation of the release characteristics of a formulation corresponding to a total weight of $450 \mathrm{mg}$ with 100 of drug was also performed to determine if its release characteristics approached better the ones of the 500-mg tablet with $200 \mathrm{mg}$. The T50\% and T90\% for this formulation were 1.9 and 6.4 hours (Table 4.5), very similar to values of the selected twice-daily SD HASCA formulation with $200 \mathrm{mg}$, and more similar than the T50\% and T90\% for the 400-mg and 500-mg tablets with $100 \mathrm{mg}$ of drug. 
Table 4.3 Time to release $50 \%$ and $90 \%$ of tramadol hydrochloride [T50\%, T90\%] from twice-daily (every 12 hours) and once-daily (every 24 hours) commercially SR formulations and SD HASCA formulations (produced with the SSP), with $100 \mathrm{mg}$ and $200 \mathrm{mg}$ of drug. Release-times are presented in hours.

\begin{tabular}{|c|c|c|c|c|c|c|}
\hline \multicolumn{7}{|c|}{ Frequency of administration: twice-daily } \\
\hline \multirow[t]{5}{*}{ Formulation } & \multicolumn{3}{|l|}{ Commercial } & & \multicolumn{2}{|c|}{ SD HASCA (SSP) } \\
\hline & Topalgic $^{(B)}$ & Topalgic $^{(B)}$ & Contramal $^{(R)}$ & & \multirow{4}{*}{$\begin{array}{l}100 \mathrm{mg} \text { of } \\
\text { drug (SSP) } \\
{[3.00,} \\
10.70]\end{array}$} & \multirow{4}{*}{$\begin{array}{l}200 \mathrm{mg} \text { of } \\
\text { drug (SSP) } \\
{[3.00,} \\
10.25]\end{array}$} \\
\hline & LP 100 mg & LP $200 \mathrm{mg}$ & LP $200 \mathrm{mg}$ & & & \\
\hline & {$[2.15,7.60]$} & {$[2.00,6.90]$} & {$[2.10,6.85]$} & & & \\
\hline & & & & & & \\
\hline \multicolumn{7}{|c|}{ Frequency of administration: Once-daily } \\
\hline \multirow[t]{5}{*}{ Formulation } & Commercial & & & & \multicolumn{2}{|c|}{ SD HASCA (SSP) } \\
\hline & Tridural $^{(B)}$ & Tridural $^{(B)}$ & Ralivia $^{(B)}$ & Zytram $^{B} \mathrm{XL}$ & $100 \mathrm{mg}$ of & $200 \mathrm{mg}$ of \\
\hline & $100 \mathrm{mg}$ & $200 \mathrm{mg}$ & $200 \mathrm{mg}$ & $200 \mathrm{mg}$ & drug (SSP) & drug (SSP) \\
\hline & {$[6.10$} & {$[6.00$} & {$[6.40$} & {$[7.50,>24]$} & {$[5.20$} & {$[5.20$} \\
\hline & $15.70]$ & $19.50]$ & $11.60]$ & & 20.40] & 19.10] \\
\hline
\end{tabular}

In addition, the $f_{2}$ results also showed that the $450-\mathrm{mg}$ tablet with $100 \mathrm{mg}$ of drug presented release characteristics more similar to the selected twice-daily formulation with 200 $\mathrm{mg}$ of tramadol hydrochloride $\left(f_{2}\right.$ equal to 89.23$)$ and to Topalgic ${ }^{\circledR}$ LP $100 \mathrm{mg}\left(f_{2}\right.$ equal to 72.71), which led to the selection of the formulation weighting $450 \mathrm{mg}$ as the twice-daily SD HASCA formulation with $100 \mathrm{mg}$. Close similarity between both the twice-daily and the oncedaily developed formulations with different contents of drug was desired.

Given that SD HASCA formulations were produced for oral administration, the size of the tablets had to be taken into consideration when producing once-daily formulations. Consequently, an increase in tablet size was not considered and the tablet weighting $800 \mathrm{mg}$ with $200 \mathrm{mg}$ of drug was chosen as the once-daily formulation with $200 \mathrm{mg}$ of tramadol hydrochloride, even considering that the release rate from this tablet was faster than the rate from Tridural ${ }^{\circledR} 200 \mathrm{mg}\left(f_{2}\right.$ equal to 55.83). In addition, even though the release from the tablets weighting $800 \mathrm{mg}$ with $100 \mathrm{mg}$ of tramadol hydrochloride was slower and more similar to Tridural ${ }^{\circledR} 100 \mathrm{mg}\left(f_{2}\right.$ equal to 68.1$)$ than the tablet weighting $700 \mathrm{mg}$ with the same drug- 
content $\left(f_{2}\right.$ equal to 53.09), the latter had a profile more similar to the tablet with a total weight of $800 \mathrm{mg}$ and $200 \mathrm{mg}$ of tramadol hydrochloride ( $f_{2}$ equal to 82.39), the selected once-daily SD HASCA formulation with $200 \mathrm{mg}$ of drug. Therefore, the 700-mg tablet with $100 \mathrm{mg}$ of drug was chosen as the optimal once-daily formulation with 100 of tramadol hydrochloride.

$\mathrm{A} \mathrm{pH}$ gradient was used instead of a unique buffer or distilled water because it provides a more accurate simulation of the environment that an oral dosage form encounters when transiting through the gastrointestinal tract. Besides, studies have shown that in vivo-in vitro correlations (IVIVC) are improved when the dissolution tests are carried out in a $\mathrm{pH}$ gradient rather than in distilled water [33].

Studies have demonstrated that the DS has a significant influence on the structural, physicochemical and drug-release properties of high-amylose carboxymethyl starch $[29,34]$. These studies have suggested that high-amylose sodium carboxymethyl starches produced at lower DS are preferably suitable for use as an excipient for SR formulations, showing extended-release in both acidic and alkaline dissolution media, while high-amylose sodium carboxymethyl starch at higher DS (referred to as CM-HAS) can be used as an excipient for delayed-release, since the drug-release rate in acidic medium is significantly lower than in alkaline medium $[29,34]$. As a result, the potential of this polymer produced at higher DS as an excipient for formulations with gastro-resistant properties of a variety of drugs, including bioactive agents, have been investigated $[35,36]$. The polymer used in this study was produced at a lower DS and, therefore, shows SR properties in both acid and alkaline environment.

\subsubsection{Decrease of the tablet surface area in order to increase the in vitro release time of tramadol hydrochloride from twice-daily and once-daily SD HASCA matrix tablets}

The comparison between the T50\% and T90\% from the once-daily SD HASCA formulations with $100 \mathrm{mg}$ ( 4 and $14.4 \mathrm{~h}$ ) and $200 \mathrm{mg}$ of tramadol hydrochloride (4.1 and 16.4 h), prepared using a HP (Table 4.5), and the T50\% and T90\% from Tridural ${ }^{\circledR} 100 \mathrm{mg}(6.1$ and $15.7 \mathrm{~h}$ ) and Tridural ${ }^{\circledR} 200 \mathrm{mg}(6$ and $19.5 \mathrm{~h}$ ), the once-daily commercial dosage forms (Table 
4.3), show that the release rates from the once-daily SD HASCA tablets were slightly higher than the release rates from the commercial tablets. Closer values were, however, found for the tablets intended for twice-daily administration.

The ultimate purpose of the developed SD HASCA SR formulations is oral administration in humans. Tablet size, shape and geometry are important factors to take into consideration when designing medication to be administered by this route, since they determine the level of patient comfort during oral administration, and thus compliance. Besides, it has been shown that tablet size, shape, and surface area may affect drug-release profiles [37,38] and can, therefore, be used for modulation of drug-release rate. A study on the influence of tablet surface area/volume (SA/V) ratio on drug-release from controlled-release matrix tablets containing HPMC indicated a direct relationship between SA/V ratios and drugrelease rate. Utility of $\mathrm{SA} / \mathrm{V}$ to influence drug-release was demonstrated by altering tablet shape to adjust SA/V [37]. The importance of this finding is related to the possibility of achieving optimal drug-release profiles without further modification of a formulation, by simply choosing an appropriate $\mathrm{SA} / \mathrm{V}$ ratio for a tablet. Based on the higher comfort for the patient when swallowing tablets with narrower diameters and the direct link found between surface area and drug-release rate, the dimensions of the selected SR SD HASCA formulations were changed so as to decrease their diameter, decreasing the tablet SA/V ratio. To achieve these changes, the tablets were compressed using a SSP machine instead of the 30-tons manual HP. This compressing machine produced tablets with an average diameter of $11.1 \mathrm{~mm}$ instead of $12.6 \mathrm{~mm}$ and, consequently, tablet heights were thicker.

When using the SSP, the lower plunger nuts was adjusted so as to achieve a target average thickness of $7 \mathrm{~mm}$ for the once-daily tablet with $100 \mathrm{mg}$ of drug (instead of $4.4 \mathrm{~mm}$ of the tablets produced with the HP), $7.4 \mathrm{~mm}$ for the once-daily with $200 \mathrm{mg}$ of drug (instead of $4.8 \mathrm{~mm}$ of the tablets produced with the HP), $4.6 \mathrm{~mm}$ for the twice-daily tablets with $100 \mathrm{mg}$ and $5.2 \mathrm{~mm}$ for the twice-daily tablets with $200 \mathrm{mg}$ (no thickness measurements had been made with the HP). As the number of sample tablets was small, it was not possible to present the values of the standard deviations of the tablet thickness.

The in vitro drug-release characteristics from the tablets produced with the SSP were then evaluated under the same $\mathrm{pH}$ gradient dissolution conditions. Even though the twice-daily SD HASCA formulations presented a release profile close to the profile of the commercial 
ones, the same changes in surface area were studied so as to develop all the four formulations under the same conditions and make them all more easily swallowable.

\subsubsection{Preliminary crushing strengths measurements}

The crushing strengths of 700-mg and 800-mg tablets with $100 \mathrm{mg}$ and $200 \mathrm{mg}$ of tramadol hydrochloride, respectively, compressed with the HP were measured. The designed procedure was to apply a CF starting at 1 tons $/ \mathrm{cm}^{2}$ and to increase it gradually up to 2.5 tons $/ \mathrm{cm}^{2}$. Not even the 700-mg and the 800-mg SD HASCA tablets with $100 \mathrm{mg}$ and $200 \mathrm{mg}$ of drug, compressed with the HP at 1 ton $/ \mathrm{cm}^{2}$ were breakable. Therefore, no further measurements were necessary. The very high crushing strengths found for these tablets support the theory, described above, of the occurrence of particle re-arrangement and partial melting of the polymer under compression, resulting in the densification of the matrices. The high crushing strength values are an advantage when considering industrial manufacture because they ensure batch reproducibility throughout the process.

\subsubsection{Influence of CF on tramadol hydrochloride release from SD HASCA tablets}

The influence of CF ranging between 1 and 2.5 tons $/ \mathrm{cm}^{2}$ on the drug-release properties from SD HASCA matrix tablets weighting 700-mg tablets with $100 \mathrm{mg}$ of tramadol hydrochloride, and compressed using a 30-tons manual HP, was also investigated. This range of CF was selected because it covers the normal range of CF employed at the industrial level. The $\mathrm{T} 50 \%$ and $\mathrm{T} 90 \%$ for the tested CF and the values of the $f_{2}$ between tablets compressed at different CF are represented in Table 4.4. Between 1 and 2.5 tons $/ \mathrm{cm}^{2}$, CF did not significantly influence drug-release from SD HASCA matrix tablets. The same results were found for 600 - and 400-mg tablets containing 32.5\% of SD HASCA (with 70\% of amylose and $30 \%$ of amylopectin, and spray-dried with a final ethanol/HASCA ratio w/w of 3.2), $40 \%$ of acetaminophen and $27.5 \%$ of sodium chloride [16]. 
Table 4.4 Time to release $50 \%$ and $90 \%$ of tramadol hydrochloride [T50\%, T90\%] for SD HASCA tablets compressed at different CF between 1 and 2.5 tons $/ \mathrm{cm}^{2}$ and similarity factors $\left(f_{2}\right)$ between tablets weighting 700-mg tablets with $100 \mathrm{mg}$ of tramadol hydrochloride, compressed with a 30-tons manual HP at different CF.

\begin{tabular}{|lll|}
\hline CF $\left(\right.$ tons $\left./ \mathrm{cm}^{2}\right)$ & {$[\mathrm{T} 50 \%, \mathrm{~T} 90 \%]$ (hours) } & $f_{2}$ Vs. 2.5 tons $/ \mathrm{cm}^{2}$ \\
\hline 1 & {$[3.90,14.25]$} & 90 \\
1.5 & {$[4.10,14.50]$} & 89 \\
2 & {$[4.25,15.40]$} & 84 \\
2.5 & {$[4.00,14.40]$} & \\
\hline
\end{tabular}

These consistent results show that the independence between drug-release from SD HASCA matrices and the CF used is not affected by the different composition of the polymer, the final ethanol/HASCA ratio and the substitution of sodium chloride for tramadol hydrochloride as the electrolyte in the formulation. Ungur et al. [11] have already noted that in the case of lab-scale HASCA, CF influenced microporosity but did not alter the drug-release rate. A study using formulations composed of SD HASCA, acetaminophen and sodium chloride, where the relationship between TW and CF versus TT was investigated to understand the good binding properties of SD HASCA, suggested that an intense densification of the matrices occurred and was the same for CF ranging from 1 to 2.5 tons $/ \mathrm{cm}^{2}$. In this case, it was also demonstrated that CF does not considerably influence TT [16]. This peculiar mechanism of densification, i.e., sintering by viscous flow and melting under compression, has been demonstrated by scanning electron microscopy (SEM) and porosimetry for matrices composed of SA,G-2.7 [10]. Such very low porosity might explain why CF does not influence the drug-release rate from tablets with SD HASCA or SA,G-2.7 as the SR excipient. As opposed to SA,G-2.7, no influence of CF on the amplitude of the burst effect as well as on the time-lag has also been observed for SD HASCA tablets. 
The fact that the CF applied did not significantly influence drug-release is important since it indicates that the unknown CF exerted by the SSP machine may not have a significant influence on the release of tramadol hydrochloride from SD HASCA tablets compressed with this machine. This finding and the very high crushing strengths further illustrate the utility of SD HASCA as a directly compressible excipient for oral sustained drug-release and assures the reliableness of the SSP.

4.5.3.3 In vitro release of tramadol hydrochloride from optimal twice-daily and once-daily SD HASCA matrix tablets

As shown in Figure 4.3, the 700-mg SD HASCA tablets (once-daily) with $100 \mathrm{mg}$ of drug compressed with the SSP machine had a slightly lower release than the once-daily commercial formulations after 10 hours of release. At this stage of experimentation, this does not represent a problem given that one does not know yet how these SD HASCA dosage forms will behave in vivo.

Decreasing the diffusion surface area using the SSP led to a decrease in the release rate and consequently to an improvement of the similarity of the 700-mg tablet with $100 \mathrm{mg}$ of drug to Tridural ${ }^{\circledR} 100 \mathrm{mg}$, when compared with the same formulation compressed with the HP ( $f_{2}$ equal to 70.66 for the SSP vs. 53.09 for the HP). In the same way, the $f_{2}$ for the $800-\mathrm{mg}$ tablet with $200 \mathrm{mg}$ of drug compressed with the SSP machine was relatively higher than the $f_{2}$ for the 800 -mg tablet with $200 \mathrm{mg}$ of drug compressed with the HP, when both formulation were compared to Tridural ${ }^{\circledR} 200 \mathrm{mg}\left(f_{2}\right.$ equal to 79.80 for the SSP vs. 55.83 for the HP). 


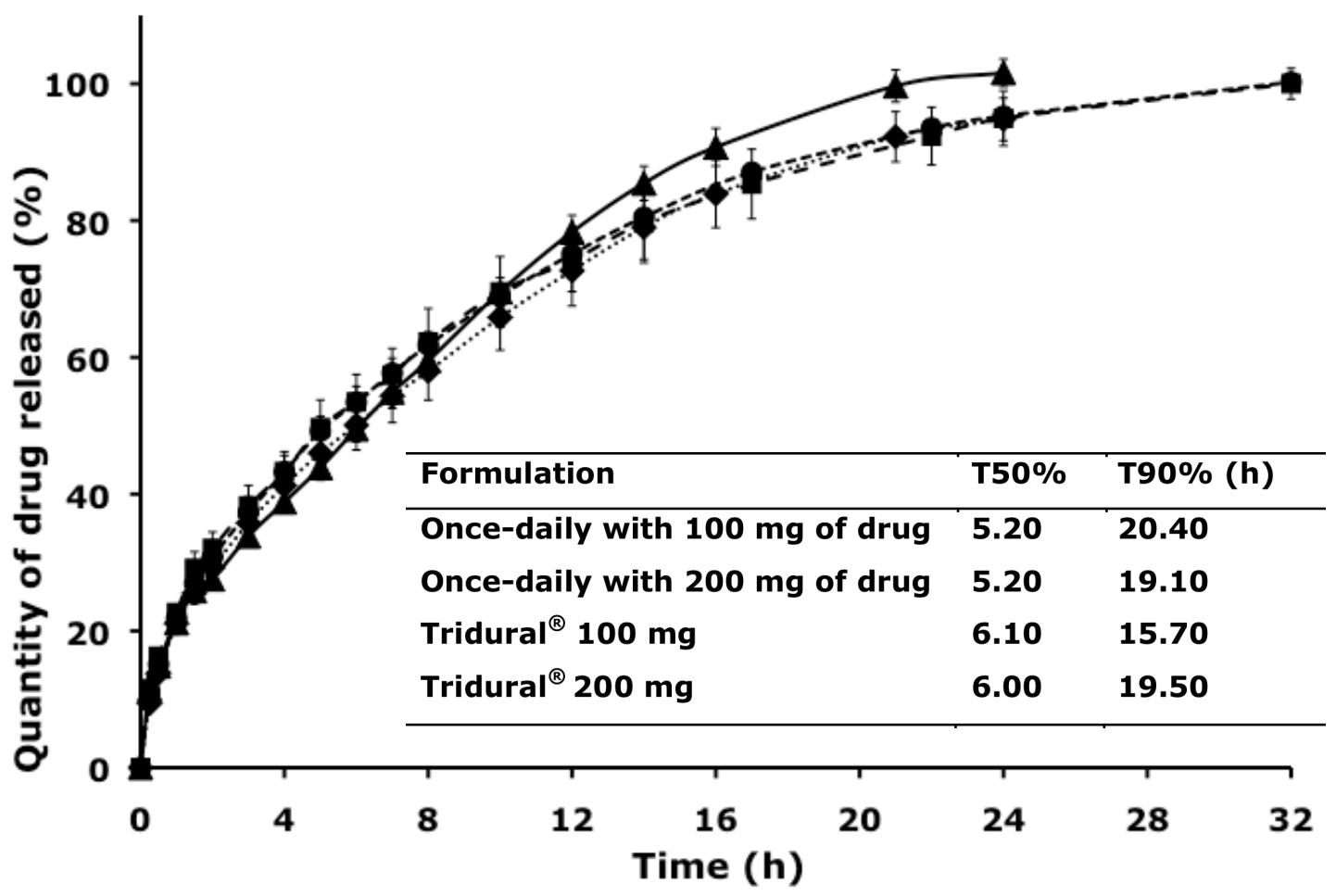

Figure 4.3 Cumulative percentage of tramadol hydrochloride released in vitro from once-daily SD HASCA formulations compressed using a single-stroke press machine (SSP), and oncedaily commercial formulations (ㅁ: 700-mg tablets with $100 \mathrm{mg}$ of drug, $\bullet$ : 800-mg tablets with $200 \mathrm{mg}$ of drug, $\boldsymbol{\Delta}$ : Tridural $^{\circledR} 100 \mathrm{mg}$, and $\diamond$ : Tridural ${ }^{\circledR} 200 \mathrm{mg}$ ). Tests were performed with the tablets placed inside a cage. $f_{2}$ values: 70.66 between $700-\mathrm{mg}$ tablets with $100 \mathrm{mg}$ and Tridural $^{\circledR} 100 \mathrm{mg}, 79.80$ between 800-mg tablets with $200 \mathrm{mg}$ and Tridural ${ }^{\circledR} 200 \mathrm{mg}, 93.14$ between 700-mg tablets with $100 \mathrm{mg}$ and 800-mg tablets with $200 \mathrm{mg}$.

The $f_{2}$ between the once-daily SD HASCA with $100 \mathrm{mg}$ and the once-daily SD HASCA with $200 \mathrm{mg}$ formulations compressed with the SSP was 93.14, much superior to the value between the commercial formulations, i.e., 69.67. Figure 4.4 shows that the release from both the 450-mg tablets with $100 \mathrm{mg}$ of drug and the 500-mg tablets with $200 \mathrm{mg}$ of drug, compressed with the SSP, was slower than the release from the commercial formulations with $100 \mathrm{mg}$ and $200 \mathrm{mg}$ ( $f_{2}$ equal to 54.52 for twice-daily SD HASCA $100 \mathrm{mg}$ and Topalgic ${ }^{\circledR}$ LP $100 \mathrm{mg}$; and equal to 52.36 for twice-daily SD HASCA $200 \mathrm{mg}$ and Topalgic ${ }^{\circledR}$ LP $200 \mathrm{mg}$ ). The $f_{2}$ regarding the similarity between the twice-daily SD HASCA formulations compressed with the SSP (88.01) and the $f_{2}$ regarding the similarity between commercial formulations (85.06) were nearly identical. The differences between the release profiles from the two types 
of twice-daily formulations, commercial and SD HASCA, were not an issue, since the main goal of the present work was essentially to prove the SR properties of the developed SD HASCA formulations and to develop once-daily and twice-daily SD HASCA formulations with similar release profiles for both dose strengths $(100 \mathrm{mg}$ and $200 \mathrm{mg})$ and type of formulation (once-daily and twice-daily). Besides, the commercial formulations were mainly a guide to remain within reliable release profiles for the 12 hours and the 24 hours release formulations. Therefore, a strict similarity was not required between SD HASCA and marketed formulations.

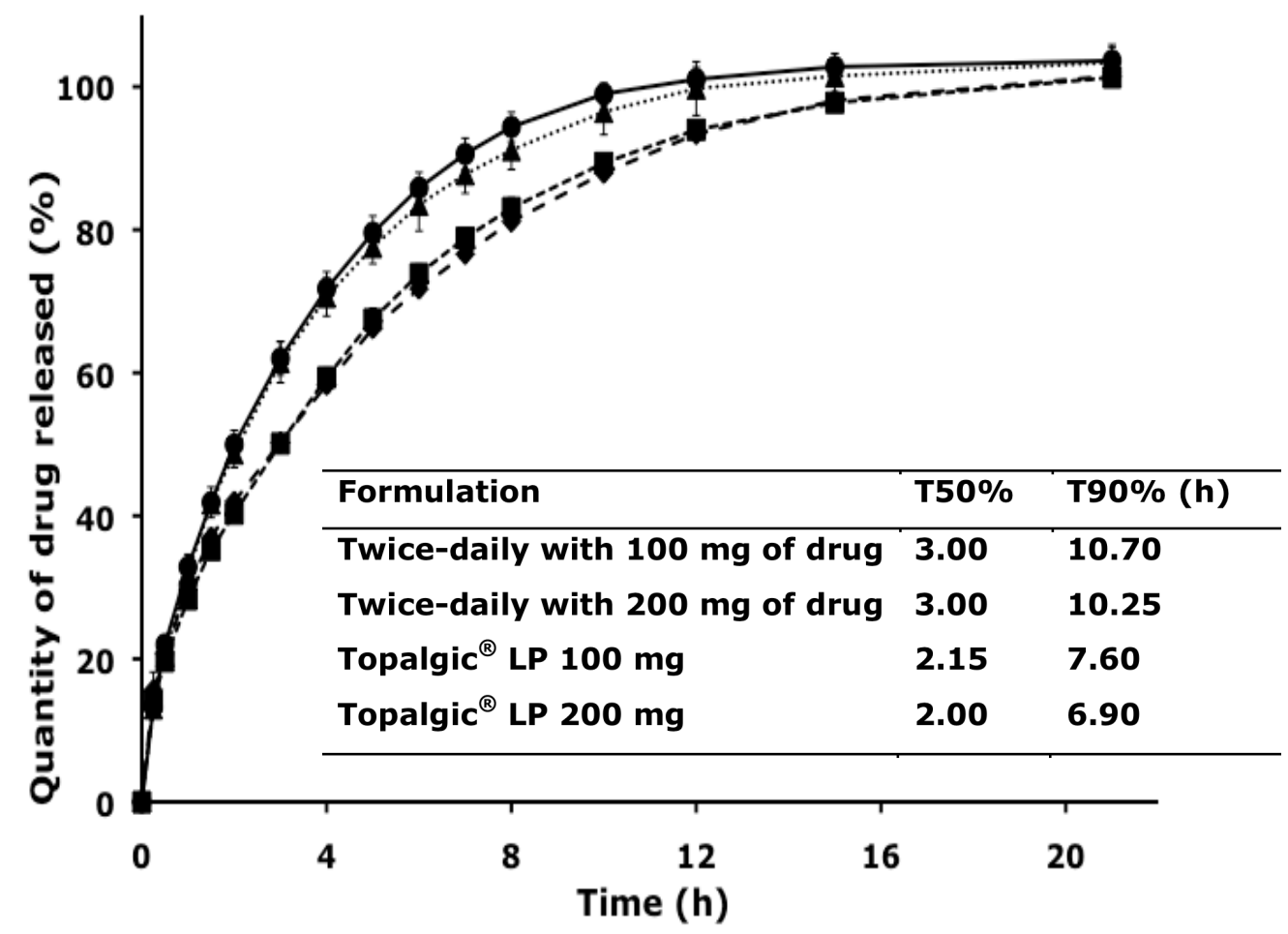

Figure 4.4 Cumulative percentage of tramadol hydrochloride released in vitro from twice-daily SD HASCA formulations, compressed with the single-stroke press machine, and twice-daily commercial formulations ( $\diamond$ : 450-mg tablets with $100 \mathrm{mg}$ of drug, $\mathbf{\square}: 500$-mg tablets with $200 \mathrm{mg}$ of drug, $\boldsymbol{\Delta}$ : Topalgic ${ }^{\circledR}$ LP $100 \mathrm{mg}$, and $\bullet$ : Topalgic ${ }^{\circledR}$ LP $200 \mathrm{mg}$ ). Tests were performed with the tablets placed inside a cage. $f_{2}$ values: 54.52 between $450-\mathrm{mg}$ tablets with $100 \mathrm{mg}$ and Topalgic ${ }^{\circledR}$ LP $100 \mathrm{mg}, 52.36$ between 500-mg tablets with $200 \mathrm{mg}$ of drug and Topalgic $^{\circledR}$ LP $200 \mathrm{mg}, 88.01$ between 450-mg tablets with $100 \mathrm{mg}$ and 500-mg tablets with $200 \mathrm{mg}$ of drug. 
As it can be observed in Figures 4.3 and 4.4, the release profiles from SD HASCA tablets can be divided into two stages, which are typical of hydrophilic matrices. First, a burst effect occurs. This burst of drug corresponds to the rapid dissolution and release of drug from the tablet surface, whilst the viscous gel layer forms around the dry core of the matrix tablet. Thereafter, the release rate decreases gradually until the end of the dissolution process. This decrease in release rate corresponds to an increase of the diffusion pathway that drug molecules have to traverse, owing to the progressively forming gel barrier. This release behaviour is characteristic of a mainly diffusion-controlled mechanism [39].

Table 4.5 shows a clear decrease in the rate of drug-release, given by the T50\% and T90\%, from twice-daily and once-daily SD HASCA tablets compressed using the SSP when compared to the tablets compressed with the HP. In the same way as it was observed for the tablets compressed with the HP, the T50\% was less affected by TW than the T90\% when the tablets were compressed using the SSP. The in vitro release rates from the optimized twicedaily and once-daily SD HASCA formulations were in agreement with documented preferred profiles [40].

Table 4.5 Time to release $50 \%$ and $90 \%$ (hours) of tramadol hydrochloride [T50\%, T90\%] from twice-daily and once-daily SD HASCA tablets with $100 \mathrm{mg}$ and $200 \mathrm{mg}$ of drug, compressed using a 30-tons manual hydraulic press at $2.5 \mathrm{tons} / \mathrm{cm}^{2}(\mathrm{HP})$ or a single-stroke press (SSP).

\begin{tabular}{|ccccc|}
\hline \multirow{2}{*}{ Frequency of } & \multicolumn{4}{c|}{ Formulation } \\
administration & $100 \mathrm{mg}$ of & $100 \mathrm{mg}$ of & $200 \mathrm{mg}$ of & $200 \mathrm{mg}$ of \\
& $\operatorname{drug}(\mathrm{HP})$ & $\operatorname{drug}(\mathrm{SSP})$ & $\operatorname{drug}(\mathrm{HP})$ & $\mathrm{drug}(\mathrm{SSP})$ \\
\cline { 2 - 5 } Twice-daily & {$[1.90,6.40]$} & {$[3.00,10.70]$} & {$[1.90,6.70]$} & {$[3.00,10.25]$} \\
$\underline{\text { Once-daily }}$ & {$[4.00,14.40]$} & {$[5.20,20.40]$} & {$[4.10,16.40]$} & {$[5.20,19.10]$} \\
\hline
\end{tabular}




\subsubsection{In vitro release of tramadol hydrochloride from SD HASCA tablets under ethanolic conditions}

The results of the in vitro release of tramadol hydrochloride from once-daily SD HASCA tablets with $100 \mathrm{mg}$ of tramadol hydrochloride and from Tridural ${ }^{\circledR} 100 \mathrm{mg}$ (oncedaily) in a hydro-alcoholic medium with $40 \%$ ethanol, performed to address safety issues related to simultaneous administration of SD HASCA tablets and ingestion of alcohol, are presented in Figure 4.5 The same figure shows the release profiles from the same formulations in a $\mathrm{pH}$ gradient. The rate of release of tramadol hydrochloride from SD HASCA tablets decreased under ethanolic conditions when compared to the rate of release in a $\mathrm{pH}$ gradient. SD HASCA formed a very hard gel in the hydro-alcoholic medium, which retained SR properties independently of the alcoholic environment. The same decline in the rate of release was observed for Tridural $^{\circledR} 100 \mathrm{mg}$, having SD HASCA and the commercial formulations remarkably similar profiles ( $f_{2}$ equal to 88.51$)$. 


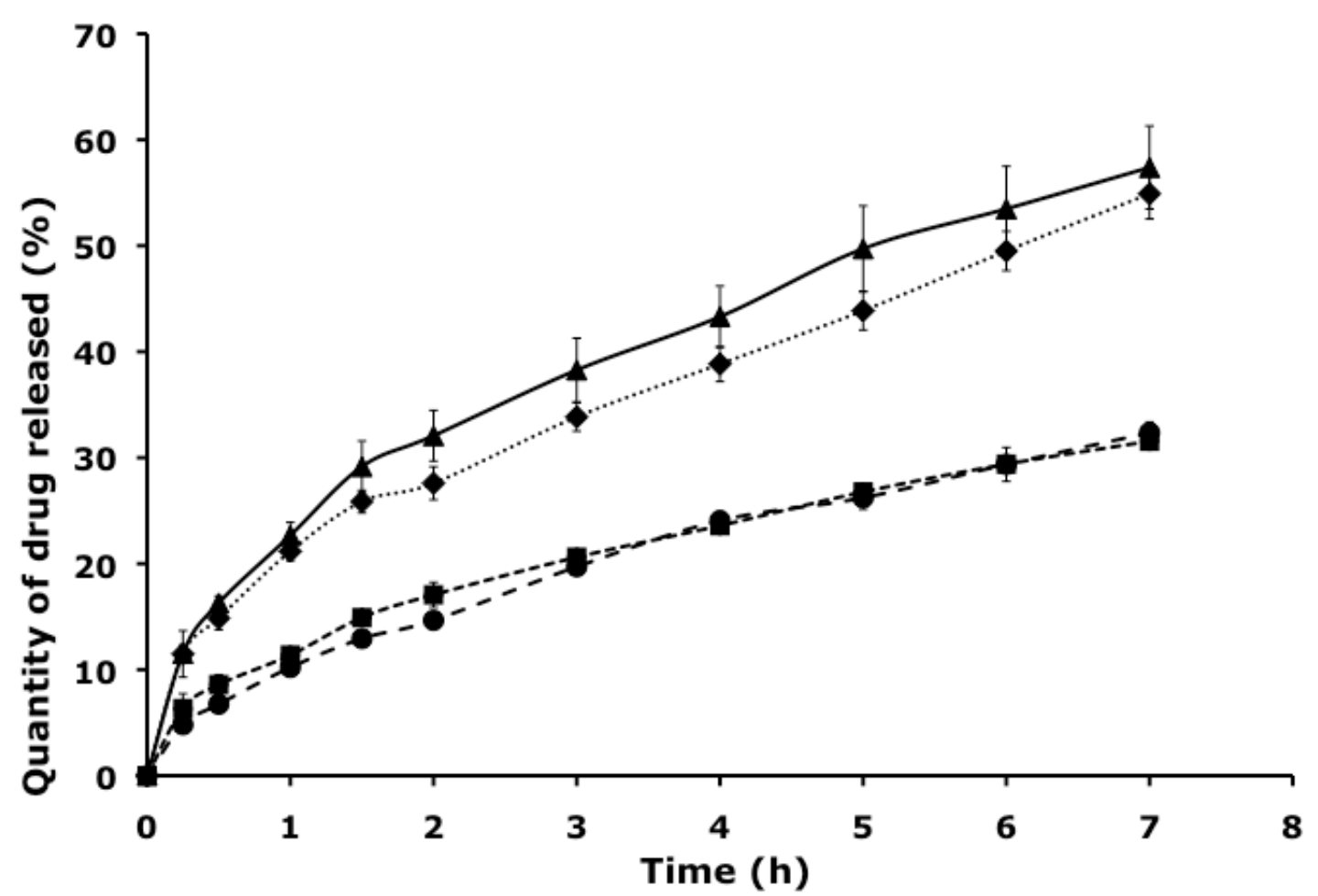

Figure 4.5 Cumulative percentage of tramadol hydrochloride released in vitro from once-daily SD HASCA tablets with $100 \mathrm{mg}$ of drug and once-daily commercial formulations with 100 $\mathrm{mg}$ of drug in a $\mathrm{pH}$ gradient versus a $40 \%$ ethanol hydro-alcoholic medium ( $\mathbf{\Delta}$ : SD HASCA with $100 \mathrm{mg}$ in a pH gradient, $\bullet$ : Tridural ${ }^{\circledR} 100 \mathrm{mg}$ in a pH gradient, $\bullet$ : SD HASCA with $100 \mathrm{mg}$ in a $40 \%$ ethanolic medium $\mathbf{\text { 口: }}$ Tridural ${ }^{\circledR} 100 \mathrm{mg}$ in a $40 \%$ ethanolic medium. $f_{2}$ values: 70.66 between 700 -mg tablets with $100 \mathrm{mg}$ of drug and Tridural ${ }^{\circledR} 100 \mathrm{mg}$ in $\mathrm{pH}$ gradient, 88.51 between $700-\mathrm{mg}$ tablets with $100 \mathrm{mg}$ of drug and Tridural ${ }^{\circledR} 100 \mathrm{mg}$ in a $40 \%$ ethanol hydro-alcoholic medium.

It has been reported that in vitro dissolution experiments in $40 \%$ ethanol hydroalcoholic mediums over a 2 hours period are expected to be representative of the most extreme alcoholic conditions likely to be encountered in vivo, because of to the dilution of the ethanol in the gastrointestinal fluids [26]. Yet, the dissolution tests in alcohol were carried out during a 7 hours period to better distinguish the different drug-release profiles between formulations and between dissolution mediums.

The decline in the release rate may be explained by a possible susceptibility of the solubility of SD HASCA, the SR agent in our tablets, and Contramid ${ }^{\circledR}$ (a cross-linked starch), 
the SR agent in Tridural ${ }^{\circledR}$, to high-alcohol concentrations. Indeed, it has been shown that in heated aqueous solutions containing some alcohols, including ethanol, amylose precipitates forming complexes known as Vh-amylose crystals [41]. However, in the same study where SD HASCA was designed, it was suggested that the presence of a Vh form of HASCA was unnecessary to obtain sustained drug-release and that its concentration did not influence the drug-release process, as long as it remained a minor component in a mainly amorphous matrix [6]. Further studies on the complex interactions between ethanol and the solubility of the SR excipients are required to explain the slower release rate. Although the solubility of tramadol hydrochloride is $\mathrm{pH}$-independent, a distinct solubility of tramadol hydrochloride in a $\mathrm{pH}$ gradient versus in the ethanolic medium, if any, will also influence its release from the formulations.

Tramadol hydrochloride is available from a number of companies as once-daily SR formulations in strengths up to $300 \mathrm{mg}$. The higher dose in SD HASCA formulations is 200 $\mathrm{mg}$. These amounts of drug can possibly be released as a bolus, known as dose dumping, if misuse of the tablets by bisection, breaking, crushing, chewing or simultaneous ingestion of alcohol occurs, which could lead to harmful adverse effects like nausea, dizziness, vomiting, headache and abdominal pain [42]. Besides, tramadol adverse affects are accentuated in the presence of alcohol.

The influence of ethanol on the in vitro release of some opioid drugs, including tramadol hydrochloride, from a number of SR formulations employing diverse release technologies has been investigated following the withdrawn of a once-daily formulation of an opioid drug from the US market. Studies had shown that simultaneous ingestion of ethanol modified its release characteristics and induced dose-dumping, which urged the FDA to consider the issue of potential and harmful changes of the release characteristics of new and marked extended-release formulations caused by ethanol ingestion [26].

The dissolution studies showed that SD HASCA tablets formed a hard gel, which maintained SR properties and was resistant to exposure to a simulation of the $\mathrm{pH}$ variations through the gastrointestinal tract as well as to ethanolic conditions. Both SD HASCA and commercial formulations formed a denser gel in ethanolic medium, although the diameter and appearance of the formulations after 7 hours release did not change much (Fig. 4.6). Moreover, the moderate cracks that appeared on the tablet surface during the dissolution tests 
in a $\mathrm{pH}$ gradient did not affect drug-release, which suggests that the risk of undesired burst release in vivo might be low.
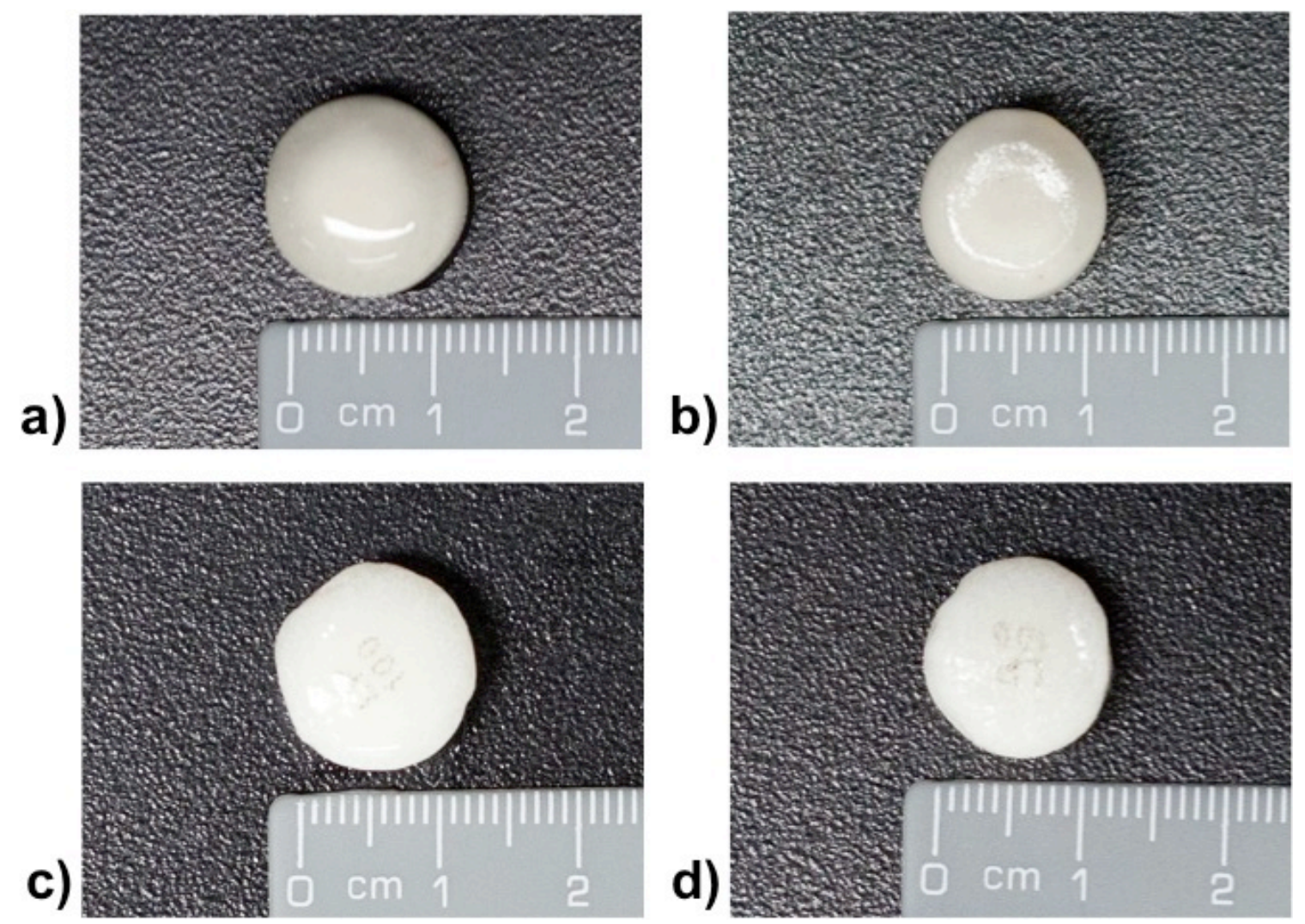

Figure 4.6 700-mg tablets with $100 \mathrm{mg}$ of tramadol hydrochloride in: (a) $\mathrm{pH}$ gradient and (b) $40 \%$ ethanol hydro-alcoholic medium (b); Tridural ${ }^{\circledR} 100 \mathrm{mg}$ in: (c) pH gradient and (d) $40 \%$ ethanol hydro-alcoholic medium.

In fact, an in vivo study where SD HASCA tablets containing acetaminophen and sodium chloride were administered to fasted, healthy human volunteers suggested that the formed gel is strong enough to maintain its integrity while traversing the stomach, resisting the vigorous peristaltic contractions which, in the fasted state, clear the stomach of any residual material (commonly knows as housekeeper waves) [17]. However, this in vivo study was carried out on a very small number of volunteers, and thus its results have to be considered carefully. Moreover, the very high crushing strengths observed for these tablets are likely to 
prevent possible accidental breaking and crushing. These findings support the value of SD HASCA as a robust excipient for oral sustained drug-release, which may reduce the likelihood of severe adverse affects that can occur in the case of an accidental dose-dumping due to misuse (breaking, crushing, bisection of the tablets or simultaneous alcohol ingestion) of the medication.

\subsection{Conclusions}

SD HASCA is an interesting excipient for sustained drug-release in solid oral dosage forms. It is biodegradable, inexpensive and allows facile manufacturing of tablets by direct compression. Tablets made from SD HASCA show independence of drug-release from CF, high crushing strengths, and are amenable to twice-daily and once-daily formulations. The mechanical strength of the SD HASCA gel formed when the tablets are exposed to either a $\mathrm{pH}$ gradient or to an ethanolic medium suggest that these tablets are likely to withstand the mechanical stresses that occur after oral administration as well as co-administration with alcohol, preventing undesired disintegration of the tablets in the gastrointestinal tract with consequent dose dumping. Moreover, the properties exhibited by SD HASCA tablets suggest this polymer is a promising and robust excipient for oral sustained drug-release, which may possibly minimize the likelihood of dose dumping and consequent adverse effects, if inadvertent or deliberate misuse of the medication such as simultaneous ingestion of alcohol, occurs.

\subsection{Acknowledgements}

This paper is dedicated to the living memory of Prof. Louis Cartilier. Teresa Nabais gratefully acknowledges the scholarship support of Fundação para a Ciência e Tecnologia (Portugal). 


\subsection{References}

[1] J. Kost, S. Shefer, Chemically-modified polysaccharides for enzymatically-controlled oral drug delivery, Biomaterials 11 (1990) 695-698.

[2] C.G. Biliaderis, The structure and interactions of starch with food constituents, Can. J. Physiol. Pharmacol. 69 (1991) 60-78.

[3] J.M. Fang, P.A. Fowler, C. Sayers, P.A. Williams, The chemical modification of a range of starches under aqueous reaction conditions, Carbohydr. Polym. 55 (2004) 283-289.

[4] E. Svensson, A.-C. Eliasson, Crystalline changes in native wheat and potato starches at intermediate water levels during gelatinization, Carbohydr. Polym. 26 (1995) 171-176.

[5] A. Rajan, V.S. Prasad, T. Emilia Abraham, Enzymatic esterification of starch using recovered coconut oil, Int. J. Biol. Macromol. 39 (2006) 265-272.

[6] F. Brouillet, G. Baylac, L. Cartilier, B. Bataille, High-amylose sodium carboxymethyl starch matrices for oral, sustained drug release: development of a spray drying manufacturing process, Drug Dev. Ind. Pharm. 36 (2010) 795-805.

[7] L. Cartilier, I. Moussa, C. Chebli, S. Buczkowski, Substitude amylose as a matrix for sustained drug release, U.S. Patent 5,879,707 (1999).

[8] C. Chebli, I. Moussa, S. Buczkowski, L. Cartilier, Substituted amylose as a matrix for sustained drug release, Pharm. Res. 16 (1999) 1436-1440.

[9] C. Chebli, L. Cartilier, Effect of some physical parameters on the sustained drug-release properties of substituted amylose matrices, Int. J. Pharm. 193 (2000) 167-173.

[10] S.H. Moghadam, H.W. Wang, E. Saddar El-Leithy, C. Chebli, L. Cartilier, Substituted amylose matrices for oral drug delivery, Biomed. Mater. 2 (2007) S71-77.

[11] M. Ungur, N. Yonis, C. Chebli, L. Cartilier, The evaluation of carboxymethylamylose for oral drug delivery systems: from laboratory to pilot scale, in: Books of Abstracts of the ISAB2-2005 3rd International Symposium on Advanced Biomaterials/Biomechanics, Montreal, Canada, 2005, pp. 271.

[12] L. Cartilier, M. Ungur, C. Chebli, Tablet Formulation for Sustained Drug-release, Canadian Patent Application 2491665 (2005). 
[13] S. Edge, R.W. Miller, Sodium starch glycolate, in: R.C. Rowe, P.J. Sheskey, S.C. Owen (Eds.), Handbook of pharmaceutical excipients, Pharmaceutical Press, London-Chicago, 2005, pp. 701-704.

[14] V. Lenaerts, I. Moussa, Y. Dumoulin, F. Mebsout, F. Chouinard, P. Szabo, M.A. Mateescu, L. Cartilier, R. Marchessault, Cross-linked high amylose starch for controlled release of drugs: recent advances, J. Control. Release 53 (1998) 225-234.

[15] F. Brouillet, B. Bataille, G. Baylac, L. Cartilier, High-amylose sodium carboxymethyl starch sustained release excipient and process for preparing the same, U.S. Patent 20100113619 A1 (2010).

[16] F. Brouillet, B. Bataille, L. Cartilier, High-amylose sodium carboxymethyl starch matrices for oral, sustained drug-release: Formulation aspects and in vitro drug-release evaluation, Int. J. Pharm. 356 (2008) 52-60.

[17] T. Nabais, F. Brouillet, S. Kyriacos, M. Mroueh, P. Amores da Silva, B. Bataille, C. Chebli, L. Cartilier, High-amylose carboxymethyl starch matrices for oral sustained drugrelease: In vitro and in vivo evaluation, Eur. J. Pharm. Biopharm. 65 (2007) 371-378.

[18] L.J. Scott, C.M. Perry, Tramadol: a review of its use in perioperative pain, Drugs 60 (2000) 139-176.

[19] S. Nossol, M. Schwarzbold, T. Stadler, Treatment of pain with sustained-release tramadol 100, 150, $200 \mathrm{mg}$ : results of a post-marketing surveillance study, Int. J. Clin. Pract. 52 (1998) 115-121.

[20] S. Grond, A. Sablotzki, Clinical pharmacology of tramadol, Clin. Pharmacokinet. 43 (2004) 879-923.

[21] R.L. Barkin, Extended-release Tramadol (ULTRAM ER): a pharmacotherapeutic, pharmacokinetic, and pharmacodynamic focus on effectiveness and safety in patients with chronic/persistent pain, Am. J. Ther. 15 (2008) 157-166.

[22] P.I. Hair, M.P. Curran, S.J. Keam, Tramadol extended-release tablets, Drugs 66 (2006) 2017-2027.

[23] H. Malonne, B. Sonet, B. Streel, S. Lebrun, S. De Niet, A. Sereno, F. Vanderbist, Pharmacokinetic evaluation of a new oral sustained release dosage form of tramadol, Br. J. Clin. Pharmacol. 57 (2004) 270-278. 
[24] M. Raber, H.U. Schulz, M. Schurer, S. Krupp, H. Momberger, Pharmacokinetic properties of tramadol sustained release capsules. 3rd communication: investigation of relative bioavailability under steady state conditions, Arzneimittelforschung 49 (1999) 594-598.

[25] USP 36 - NF 31, (United States Pharmacopeia 36 - National Formulary 31), U.S. Pharmacopeial Convention, Rockville, MD, 2013.

[26] M. Walden, F.A. Nicholls, K.J. Smith, G.T. Tucker, The effect of ethanol on the release of opioids from oral prolonged-release preparations, Drug Dev. Ind. Pharm. 33 (2007) 11011111.

[27] T. Durig, R. Fassihi, Evaluation of floating and sticking extended release delivery systems: an unconventional dissolution test, J. Control. Release 67 (2000) 37-44.

[28] J.W. Moore, H.H. Flanner, Mathematical comparison of dissolution profiles, Pharm. Tech. 20 (1996) 64-74.

[29] M. Lemieux, P. Gosselin, M.A. Mateescu, Carboxymethyl high amylose starch as excipient for controlled drug release: Mechanistic study and the influence of degree of substitution, Int. J. Pharm. 382 (2009) 172-182.

[30] N.A. Peppas, J.J. Sahlin, A simple equation for the description of solute release. III. Coupling of diffusion and relaxation, Int. J. Pharm. 57 (1989) 169-172.

[31] P. Colombo, R. Bettini, P. Santi, N.A. Peppas, Swellable matrices for controlled drug delivery: gel-layer behaviour, mechanisms and optimal performance, Pharm. Sci. Technol. Today 3 (2000) 198-204.

[32] B. Narasimhan, N.A. Peppas, Molecular analysis of drug delivery systems controlled by dissolution of the polymer carrier, J. Pharm. Sci. 86 (1997) 297-304.

[33] V.V. Ranade, Drug delivery systems 5A. Oral drug delivery, J. Clin. Pharmacol. 31 (1991) 2-16.

[34] M. Lemieux, P. Gosselin, M.A. Mateescu, Influence of drying procedure and of low degree of substitution on the structural and drug release properties of carboxymethyl starch, AAPS PharmSciTech. 11 (2010) 775-785.

[35] C. Calinescu, É. Nadeau, J. Mulhbacher, J.M. Fairbrother, M.-A. Mateescu, Carboxymethyl high amylose starch for F4 fimbriae gastro-resistant oral formulation, Int. J. Pharm. 343 (2007) 18-25. 
[36] L.P. Massicotte, W.E. Baille, M.A. Mateescu, Carboxylated high amylose starch as pharmaceutical excipients: Structural insights and formulation of pancreatic enzymes, Int. J. Pharm. 356 (2008) 212-223.

[37] T.D. Reynolds, S.A. Mitchell, K.M. Balwinski, Investigation of the Effect of Tablet Surface Area/Volume on Drug Release from Hydroxypropylmethylcellulose ControlledRelease Matrix Tablets, Drug Dev. Ind. Pharm. 28 (2002) 457-466.

[38] J. Siepmann, H. Kranz, N.A. Peppas, R. Bodmeier, Calculation of the required size and shape of hydroxypropyl methylcellulose matrices to achieve desired drug release profiles, Int. J. Pharm. 201 (2000) 151-164.

[39] Y.W. Chien, Fundamentals of controlled drug administration, in: J. Swarbrick (Ed.), Novel Drug Delivery System, Marcel Dekker, New York, 1982, pp. 465-574.

[40] B. Oshlack, H.-p. Huang, M. Chasin, P. Goldenheim, Stabilized sustained release tramadol formulations, U.S. Patent 6306438 B1 (2001).

[41] W. Helbert, H. Chanzy, Single crystals of V amylose complexed with n-butanol or npentanol: structural features and properties, Int. J. Biol. Macromol. 16 (1994) 207-213.

[42] I. Tagarro, J. Herrera, C. Barutell, M.C. Diez, M. Marin, D. Samper, C. Busquet, M.J. Rodriguez, Effect of a simple dose-escalation schedule on tramadol tolerability: assessment in the clinical setting, Clin. Drug Investig. 25 (2005) 23-31. 


\section{Chapter 5. Spray-dried high-amylose sodium carboxymethyl starch: impact of $\alpha$-amylase on drug- release profile}

Teresa Nabais ${ }^{a}$ and Grégoire Leclair ${ }^{\mathrm{a}, *}$

${ }^{a}$ Faculty of Pharmacy, University of Montreal, Montreal (Quebec) Canada;

* Corresponding author: Faculty of Pharmacy, University of Montreal, Montreal (Quebec)

Canada

Keywords: Drug delivery; Sustained release; Excipient; Polymer; Tablet; Matrix; Starch; Amylose; $\alpha$-Amylase; In vitro 


\subsection{Presentation of the article and contribution of the authors}

This article presents the results of the evaluation of influence of $\alpha$-amylase on the in vitro drug-release profiles and mechanisms from a once-daily acetaminophen formulation (Acetaminophen SR) and a once-daily tramadol hydrochloride formulation (Tramadol SR), both containing SD HASCA as the only SR excipient. In addition, the results of the study regarding the influence of the residence time in simulated gastric fluid on the $\alpha$-amylasecatalyzed erosion of both SD HASCA formulations are also described. This article was submitted to ISRN Pharmaceutics on January 22, 2014.

The contribution of each author to the publication was:

Teresa Nabais - Execution of all dissolution testing; analysis of data resulting from dissolution testing; writing of the article.

Grégoire Leclair - Project coordinator and director at the time of the publication; revision of the article.

\subsection{Abstract}

Spray-dried high-amylose sodium carboxymethyl starch (SD HASCA) is a promising pharmaceutical excipient for sustained-release (SR) matrix tablets produced by direct compression, allowing the development of twice-daily and once-daily formulations.

The presence of $\alpha$-amylase in the gastrointestinal tract and the variations of the gastric residence time of non-disintegrating dosage forms may affect the presystemic metabolism and the drug-release from SD HASCA tablets. In this study, the influence of $\alpha$-amylase on the in vitro drug-release profiles and mechanisms from a once-daily acetaminophen formulation (Acetaminophen SR) and a once-daily tramadol hydrochloride formulation (Tramadol SR), both containing SD HASCA as the only SR excipient, was evaluated. The influence of the residence time in acidic medium on the $\alpha$-amylase-catalyzed erosion of both SD HASCA formulations was also investigated. $\alpha$-Amylase concentrations ranging from $0 \mathrm{IU} / \mathrm{L}$ to 20000 
IU/L did not significantly affect the drug-release profiles of acetaminophen and tramadol hydrochloride from SD HASCA tablets. Moreover, the drug-release properties from both SD HASCA formulations were not significantly different when the residence time in acidic medium was $1 \mathrm{~h}$ or $3 \mathrm{~h}$. An increase in $\alpha$-amylase concentration led to an increase of polymer erosion over drug diffusion as the main mechanism controlling drug-release, for both formulations and both residence times in acidic medium. However, the increment in $\alpha-$ amylase-catalyzed erosion was more relevant for Tramadol SR and for a residence time in acidic medium of $1 \mathrm{~h}$. As such, $\alpha$-amylase did not significantly affect the drug-release profiles from both SD HASCA SR formulations, regardless of the residence time in acidic medium even if the mechanism of release itself may be affected.

\subsection{Introduction}

Starch is a natural polymer that can be submitted to various types of modifications, such as chemical [1], physical (i.e. gelatinization) [2], or enzymatic [3] modifications. Substituted amylose (SA), a chemically modified starch produced through the reaction of high-amylose corn starch with a substituent, has been introduced as a hydrophilic excipient for orally administered sustained-release (SR) drug delivery systems. Modified starches present advantages as hydrophilic polymers for sustained drug-release, such as a relatively low cost, accessibility, biocompatibility, biodegradability and good in vivo performances [4,5].

Spray-dried high-amylose sodium carboxymethyl starch (SD HASCA) has been proposed as a suitable and nontoxic excipient for oral SR matrix tablets prepared by direct compression [6,7]. SD HASCA, a chemically-modified starch has been produced by reacting sodium chloroacetate/chloroacetic acid with high-amylose corn starch. This polymer has shown excellent in vitro sustained drug-release properties with no increase in the burst-effect at the beginning of dissolution, when used as the only SR excipient in formulations containing a considerable quantity of soluble materials. This was previously demonstrated using acetaminophen and sodium chloride and a low quantity of polymer [4,5,8]. A first in vivo study where a formulation containing SD HASCA, acetaminophen and sodium chloride 
(referred to herein as Acetaminophen SR) was administered orally to healthy human volunteers has also demonstrated extended drug absorption, showing that the tablets maintained their integrity while traversing the stomach and resisted $\alpha$-amylase biodegradation in the small intestine [5]. The model drug chosen in this clinical study was acetaminophen, which has a plasma elimination half-life $\left(t_{1 / 2}\right)$ ranging from 1 to $4 \mathrm{~h}$ [9]. Following the positive results of the Acetaminophen SR study, further development was pursued using a clinically relevant drug. Tramadol hydrochloride, a centrally acting synthetic analgesic with a half-life between 5 and $7 \mathrm{~h}[10]$ was chosen as this second model drug.

Oral formulations intended for twice-daily and once-daily administration and containing only SD HASCA and tramadol hydrochloride, a readily soluble drug, have been successfully developed. In vitro drug-release studies have shown that these SR tablets formed a gel with high mechanical strength when exposed to a series of buffers simulating the $\mathrm{pH}$ variations through the gastrointestinal tract or to an ethanolic medium, showing excellent SR properties regardless of the medium used [11].

Further findings which have proven the potential of SD HASCA as an efficient SR excipient included a considerable increase in the total drug-release time when the tablet weight (TW) increased, tablets with high crushing strengths, facilitating their production at industrial level, and no significant influence of compression force (CF) on drug-release $[4,8,11]$.

After oral administration, starch and its derivatives are digested by $\alpha$-amylases present in certain regions of the gastrointestinal tract. In humans, the digestion of starch involves initially a partial digestion by salivary amylase resulting in the degradation of the polymeric substrate into shorter oligomers, followed by further hydrolysis by pancreatic $\alpha$-amylase into maltose, maltotriose and small maltooligosaccharides in the gut. $\alpha$-Amylase is an endospecific enzyme that catalyzes the hydrolysis of $\alpha(1 \rightarrow 4)$ glycosidic bonds of starch and other polysaccharides in a random manner, reducing the molecular size of starch [12]. This enzyme is present in bacteria, fungi, plants, and in the salivary and pancreatic glands of many animals [13]. $\alpha$-Amylase is also able to degrade chemically modified starches [14-16].

A previous dissolution study on tablets produced from substituted amylose polymers (SA,G-2.7) revealed a slightly faster drug-release in the presence of $\alpha$-amylase [14]. Moreover, glucose, maltose and maltotriose, all soluble oligosaccharides, have been identified 
as products of the hydrolysis of cross-linked high-amylose starch by $\alpha$-amylase the enzyme [15].

SR dosage forms, especially those designed for once-daily administration, contain amounts of drug that can potentially cause serious adverse effects if released too quickly in the gastrointestinal tract. This potential risk added to the fact that $\alpha$-amylase present in the gastrointestinal tract may potentially influence the hydrolysis of starch-based excipients for pharmaceutical tablets and, thereby, lead to drug-release rates different from the desired rates, makes the study of the influence of this enzyme on drug-release from SD HASCA formulations of essential importance.

In this study we propose to evaluate the impact of $\alpha$-amylase-catalyzed hydrolysis of tablets containing SD HASCA on the in vitro drug-release profiles of a once-daily acetaminophen formulation (Acetaminophen SR) and a once-daily tramadol hydrochloride formulation (Tramadol SR), as well as the influence of the residence time in acidic $\mathrm{pH}$ on the degree of hydrolysis by $\alpha$-amylase.

\subsection{Materials and methods}

\subsubsection{Materials}

Amorphous HASCA were provided in powder by Roquette Frères (Lestrem, France), and contained $65 \% \pm 5 \%$ of amylose and $35 \% \pm 5 \%$ of amylopectin. The degree of substitution (DS) of the polymers was equal to 0.045 (number of moles of substituent/number of moles of anhydroglucose) [4]. Acetaminophen was purchased from Laboratoires Denis Giroux inc. (St-Hyacinthe, Quebec, Canada), sodium chloride (crystals, lab grade) from Anachemia Ltd. (Montreal, Quebec, Canada) and tramadol hydrochloride from Jubilant Organosys Ltd. (Nanjangud, Mysore, India). All chemicals were of reagent grade and were used without further purification. $\alpha$-Amylase (EC 3.2.1.1) from Bacillus species (1260 IU/mg) was supplied by Sigma (St. Louis, MO, USA). 


\subsubsection{Preparation of SD HASCA formulations}

SD HASCA was prepared from amorphous HASCA as previously described [8]. Tablets were prepared by direct compression, i.e., blending the ingredients in a mortar using a pestle and then compression.

Acetaminophen SR tablets $(240 \mathrm{mg}$ ) were prepared from a blend of acetaminophen (40\%), sodium chloride (27.5\%) and SD HASCA (32.5\%). This blend was compressed to a target weight of $600 \mathrm{mg}$ at 2.5 tons $/ \mathrm{cm}^{2}$ for 20 seconds using a 30-ton manual hydraulic press (C-30 Research \& Industrial Instruments Company, London, U.K.) equipped with round flatfaced tooling (diameter $12.6 \mathrm{~mm}$ ). Tramadol SR tablets $(200 \mathrm{mg}$ ) were prepared from a blend of tramadol hydrochloride (25\%) and SD HASCA (75\%). This blend was compressed to a target weight of $800 \mathrm{mg}$ and a target thickness of $7.4 \mathrm{~mm}$ using a single-stroke press machine (Manesty F3 Machine, Manesty Machines Ltd., Liverpool, UK) equipped with round flatfaced tooling (diameter $11.1 \mathrm{~mm}$ ).

\subsubsection{Drug-release evaluation}

\subsubsection{Dissolution studies}

The dissolution profiles of SD HASCA SR matrix tablets in simulated gastric fluid (stage I) and simulated intestinal fluids (stage II and stage III) were evaluated using a USP apparatus $2\left(900 \mathrm{~mL}, 37^{\circ} \mathrm{C}, 50 \mathrm{rpm}\right)$ [17]. During stage I, the tablets were exposed to simulated gastric fluid conditions without enzymes (SGF, pH 1.2), for periods of 1 or $3 \mathrm{~h}$. During stage II the tablets were exposed to phosphate buffer ( $\mathrm{pH}$ 6.8) simulating the $\mathrm{pH}$ in the jejunum (SIF I) and containing $\alpha$-amylase (0, 5,000 or 20,000 IU/L), for an additional $3 \mathrm{~h}$. Levels of $\alpha$-amylase were based on a previous biodegradation study [15]. Finally, the tablets were exposed to a second phosphate buffer ( $\mathrm{pH}$ 7.4) with no enzymes, simulating the $\mathrm{pH}$ in the ileum (SIF II), until the end of the assay (total time of $24 \mathrm{~h}$ ). All standard buffer solutions were prepared according to the USP. 
Acetaminophen SR tablets were placed on stainless steel meshes positioned at the bottom of the dissolution vessels to avoid adhesion of the tablets to the vessel [5]. Similarly, Tramadol SR tablets were placed inside perforated cylindrical plastic cages to avoid eventual floating to the surface of the medium or adhesion to the vessel [11].

Each experiment was performed in triplicate. After each sampling, an equivalent volume of fresh medium was added. Samples were filtered through syringe filters $(0.45 \mathrm{~mm}$, nylon). Release was followed by UV spectrometry. The drug-release results are expressed as cumulative percentage $(\%)$ in function of time $(\mathrm{h})$.

\subsubsection{Statistical analysis and release profiles comparison}

The arithmetic mean and the standard deviation of the cumulative $\%$ of drug released at predetermined time intervals were calculated.

The dissolution profiles were compared using a similarity factor $f_{2}$ as proposed by Moore and Flanner [18]:

$$
f_{2}=50 \times \log \left\{\left[1+(1 / n) \sum_{t=1}^{n}\left(R_{t}-T_{t}\right)^{2}\right]^{-0.5} \times 100\right\}
$$

where $R_{t}$ and $T_{t}$ are the percent dissolved of the reference and test products at each time point $t$ and $n$ is the number of pool points. Two dissolution profiles are similar if $f_{2}$ is between 50 and 100 (according to the Food and Drug Administration and the European Agency for the Evaluation of Medical Products). However, the closer to 100, the more similar the dissolution profiles are. The dissolution profiles were also compared using the values of the time to release $25 \%$ (T25\%), 50\% (T50\%) and $90 \%$ of drug (T90\%). 


\subsubsection{Drug-release mechanism characterization}

Drug-release from SD HASCA tablets is controlled by a combination of Fickian diffusional release and relaxation/erosion of the polymer chains (case-II relaxational release), even in the absence of $\alpha$-amylase [4]. Moreover, in mediums containing $\alpha$-amylase, enzymecatalyzed polymer erosion might play a major role in the mechanism of drug-release. Peppas at al. [19] proposed a two-component model for this problem as detailed in Eq. (2), where the first term models the Fickian diffusion contribution $(F)$ and the second term models the relaxation/erosion $(\mathrm{R})$ contribution.

$$
M_{t} / M_{\infty}=k_{1} t^{m}+k_{2} t^{2 m}
$$

where $M_{t}$ is the cumulative amount of drug released at time $t, M_{\infty}$ is the total cumulative amount of drug released, $M_{t} / M_{\infty}$ is the fraction of drug released, $k_{1}$ is the Fickian kinetic constant and $k_{2}$ is the relaxational/erosion kinetic constant, and $m$ represents the purely Fickian diffusional exponent.

The exponent $m$ was determined from the aspect ratio, $2 a / l$ (where $2 a$ is the diameter and $l$ is the thickness ), of the tablets as described previously [19].

Eq. (2) can be used to analyze the first $60 \%$ of a release profile, regardless of geometric shape. Therefore, this equation was fitted to the experimental dissolution data, consisting of the fraction of drug released up to $M_{t} / M_{\infty}=0.6$. This fitting was calculated by nonlinear regression using the Wolfram Mathematica ${ }^{\circledR} 8$ software (Wolfram Research, Inc., Champaign, IL), in order to determine the values of the kinetic constants $k_{1}$ and $k_{2}$, and of the correlation coefficient, $R^{2}$, for each dissolution profile.

According to Peppas at al. [19] Eq. (3) leads to the ratio of erosion over Fickian contribution, as follows:

$$
R / F=\left(k_{2} / k_{1}\right) t^{m}
$$


where $R$ and $F$ are the percentage of drug released due to the relaxational/erosion and Fickian mechanism, respectively.

The ratio $k_{2} / k_{1}$ was, thereby, used to analyze the mechanism of drug-release and the quality of the fitting was given by $R^{2}$.

\subsection{Results and discussion}

\subsubsection{Influence of $\alpha$-amylase and of the residence time in SGF on the drug- release profiles from SD HASCA tablets}

5.5.1.1 $\alpha$-Amylase does not significantly affect the drug-release profiles

In view of the high doses of drug that SR dosage forms may contain and the possible increase in drug-release rate from SD HASCA tablets caused by $\alpha$-amylase present in the gastrointestinal tract, dissolution tests using SIF fluids with enzymes were essential to further address the potential of this new excipient. Indeed, dissolution tests with $\alpha$-amylase are regularly performed in the pharmaceutical industry.

Figures 5.1 (A) to 5.2 (B) show the effect of $\alpha$-amylase concentration on the in vitro drug-release from Acetaminophen SR and from Tramadol SR [5,11]. 


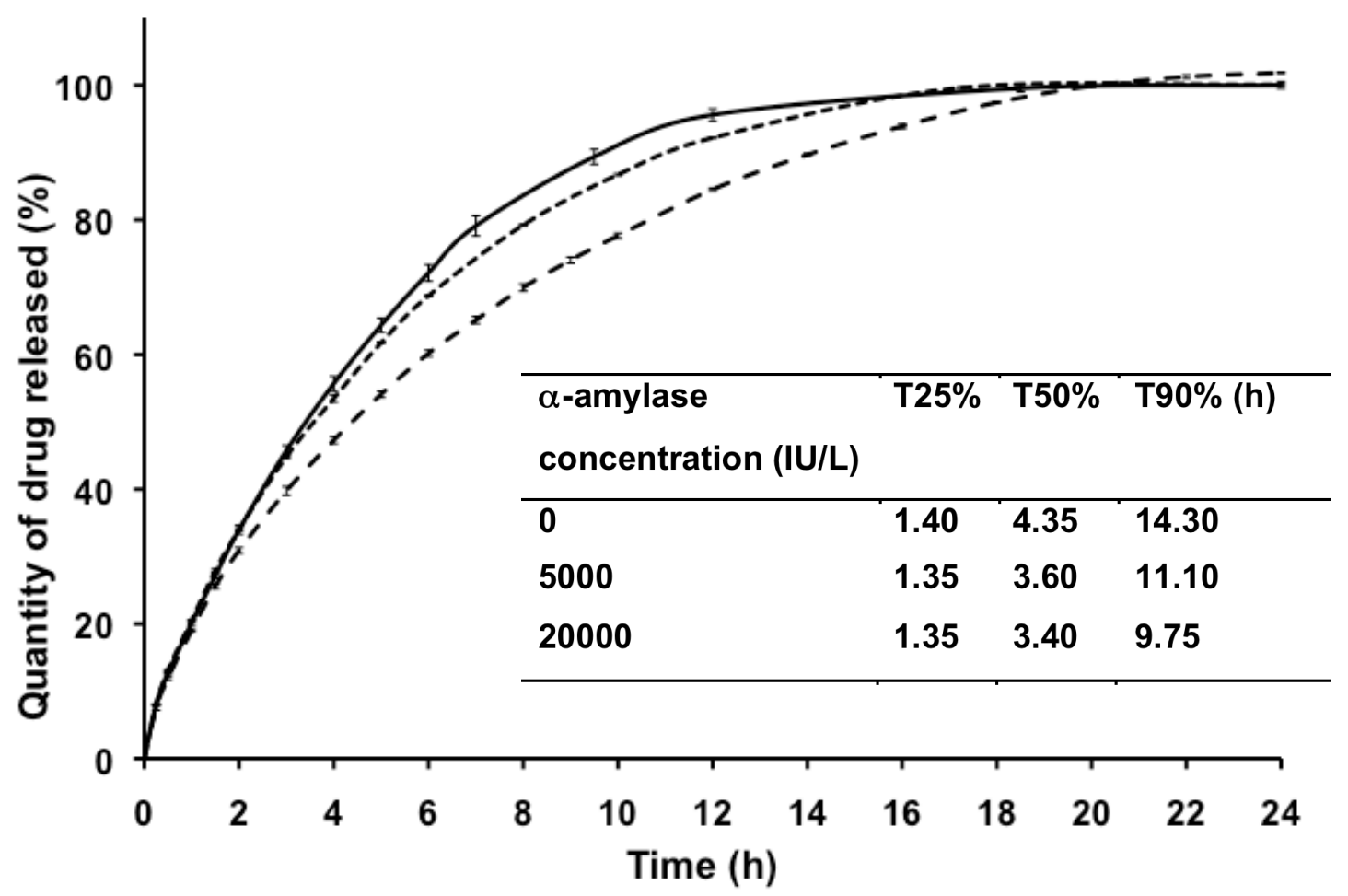

Figure 5.1 (A) Effect of $\alpha$-amylase concentration on the release of acetaminophen from Acetaminophen SR in a simulated gastrointestinal $\mathrm{pH}$, where the residence time in simulated gastric fluid ( $\mathrm{pH} 1.2$ ) was $1 \mathrm{~h}$, and the concentrations of $\alpha$-amylase in simulated intestinal fluid (pH 6.8) were: 0 IU/L (dashed line), 5000 IU/L (dotted line) and 20000 IU/L (continuous line). 


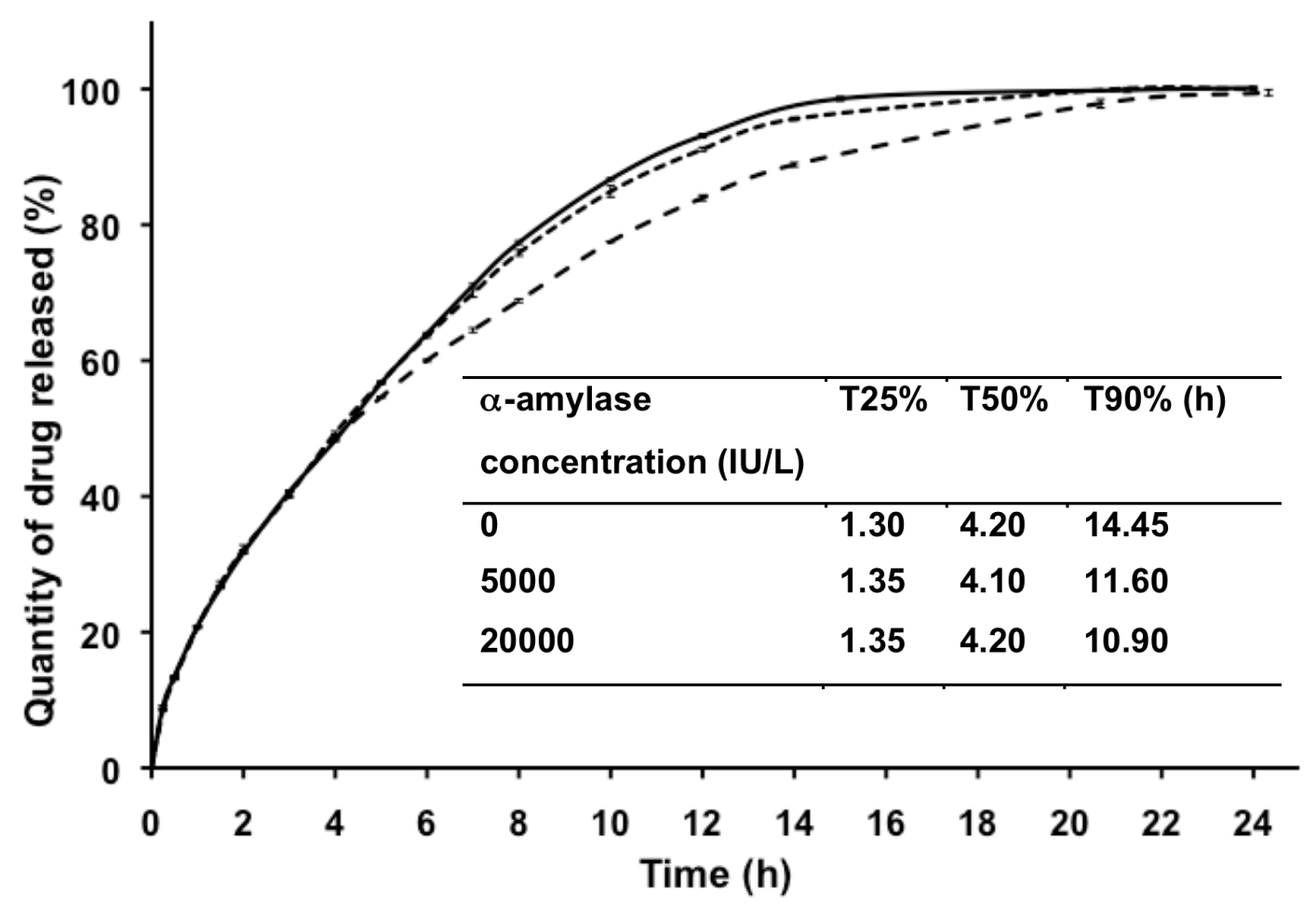

Figure 5.1 (B) Effect of $\alpha$-amylase concentration on the in vitro release of acetaminophen from Acetaminophen $\mathrm{SR}$ in a simulated gastrointestinal $\mathrm{pH}$, where the residence time in simulated gastric fluid ( $\mathrm{pH}$ 1.2) was $3 \mathrm{~h}$, and the concentrations of $\alpha$-amylase in simulated intestinal fluid (pH 6.8) were: 0 IU/L (dashed line), 5000 IU/L (dotted line) and $20000 \mathrm{IU} / \mathrm{L}$ (continuous line). 


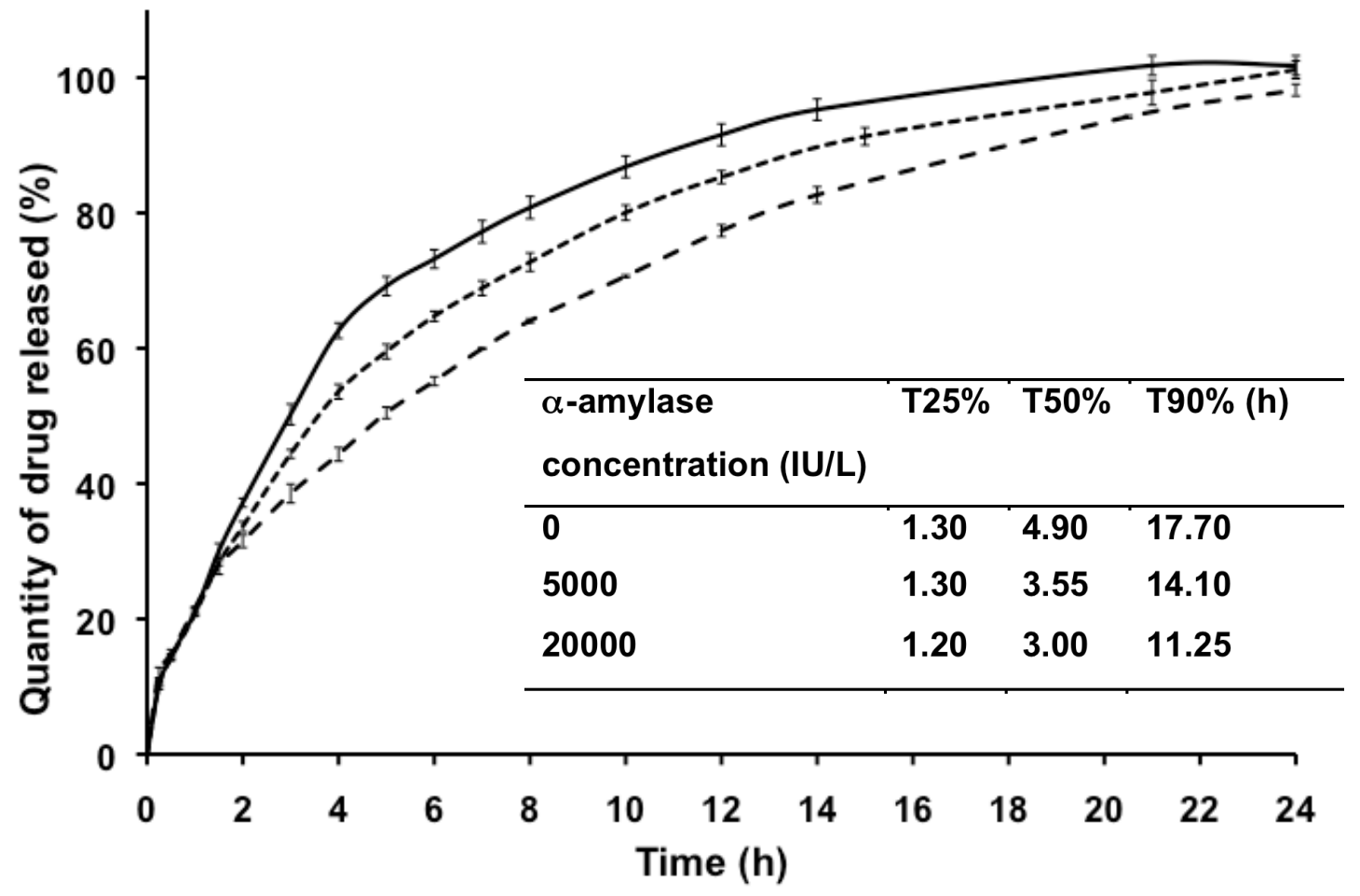

Figure 5.2 (A) Effect of $\alpha$-amylase concentration on the in vitro release of tramadol hydrochloride from Tramadol SR in a simulated gastrointestinal $\mathrm{pH}$, where the residence time in simulated gastric fluid ( $\mathrm{pH} 1.2$ ) was $1 \mathrm{~h}$, and the concentrations of $\alpha$-amylase in simulated intestinal fluid (pH 6.8) were: 0 IU/L (dashed line), 5000 IU/L (dotted line) and 20000 IU/L (continuous line). 


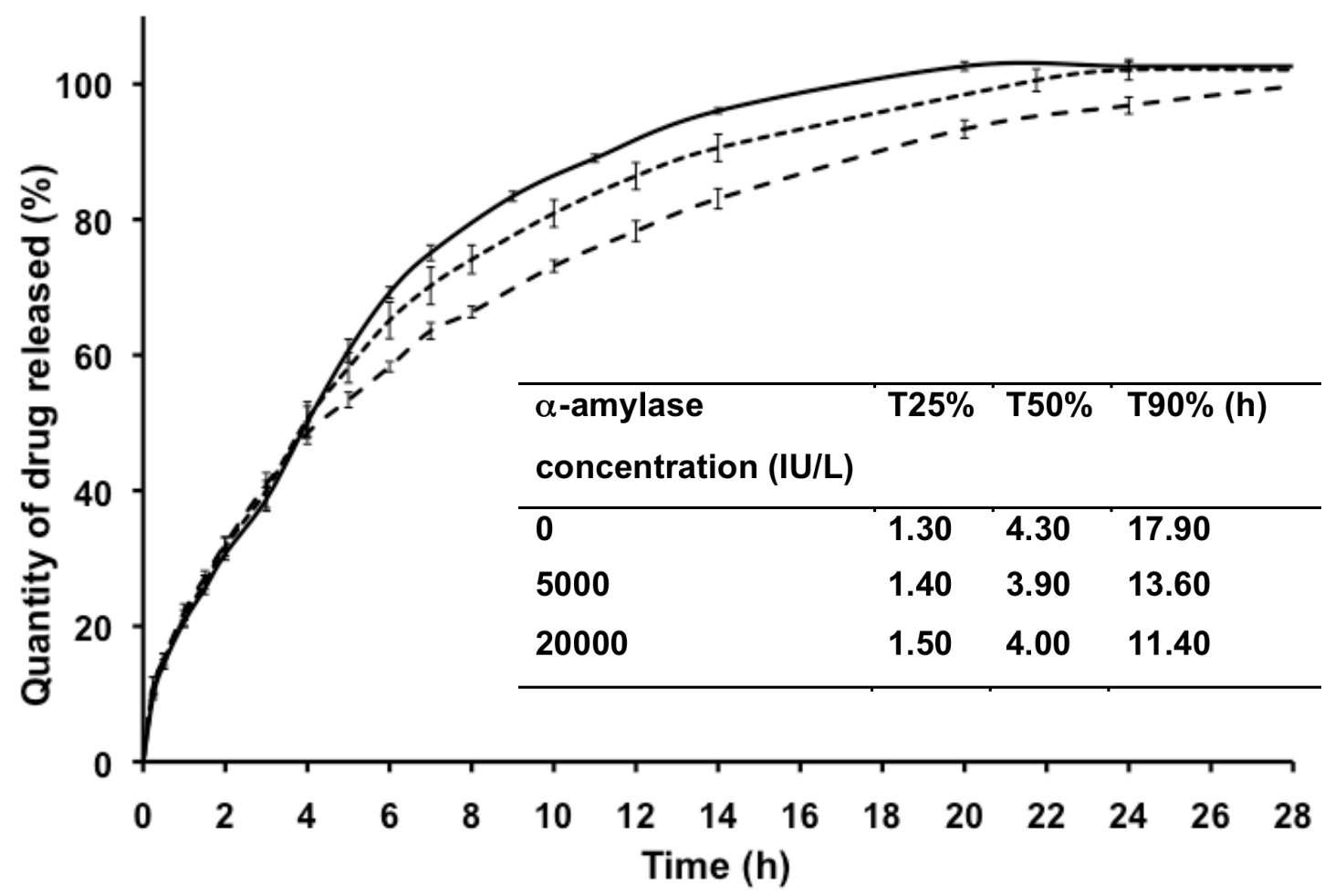

Figure 5.2 (B) Effect of $\alpha$-amylase concentration on the in vitro release of tramadol hydrochloride from Tramadol SR in a simulated gastrointestinal $\mathrm{pH}$, where the residence time in simulated gastric fluid ( $\mathrm{pH} 1.2$ ) was $3 \mathrm{~h}$, and the concentrations of $\alpha$-amylase in simulated intestinal fluid (pH 6.8) were: 0 IU/L (dashed line), 5000 IU/L (dotted line) and 20000 IU/L (continuous line).

The tablets were exposed for $3 \mathrm{~h}$ to SIF I (pH 6.8) with $0 \mathrm{IU} / \mathrm{L}, 5000 \mathrm{IU} / \mathrm{L}$ and 20000 IU/L of $\alpha$-amylase, before being transferred to SIF II ( $\mathrm{pH} 7.4)$ without $\alpha$-amylase. When immersed in SGF, a solid gel layer started forming quickly at the surface of both SD HASCA formulations. This layer developed towards the core as the series of media diffused into the tablet. Although the presence of $\alpha$-amylase in the SIF I led to a slight increase in the drugrelease rates from both SD HASCA formulations, the results, as indicated by $f_{2}$ values higher than 50 (Table 5.1), revealed that the differences between the drug-release profiles were not significant. As shown in Table 5.1, only the $f_{2}$ value between the release profiles from 
Tramadol SR at $0 \mathrm{IU} / \mathrm{L}$ and $20000 \mathrm{IU} / \mathrm{L}$ of $\alpha$-amylase, when the tablets were exposed to SGF for $1 \mathrm{~h}$, decrease to a value of 46.86. On the other hand, the highest $f_{2}$ values were observed between the release profiles from Acetaminophen SR, when the enzyme concentration rose from $5000 \mathrm{IU} / \mathrm{L}$ to $20000 \mathrm{IU} / \mathrm{L}$. A possible reason for this occurrence is the higher quantity of hydrophilic SD HASCA present in Tramadol SR $(600 \mathrm{mg})$ compared to Acetaminophen SR (195 mg), which make the first formulation more susceptible to the effect of $\alpha$-amylase.

Table 5.1 Similarity factors $\left(f_{2}\right)$ between the release profiles from Acetaminophen SR and Tramadol SR at different $\alpha$-amylase concentrations.

\begin{tabular}{|c|c|c|c|c|}
\hline \multirow[t]{3}{*}{ Formulation } & \multirow{3}{*}{$\begin{array}{l}\text { Time (h) in SGF } \\
(\mathrm{pH} 1.2)\end{array}$} & \multicolumn{3}{|c|}{$f_{2}$ between release profiles at different $\alpha$-amylase concentrations } \\
\hline & & 0 vs. 5000 & 0 vs. 20000 & 5000 vs. 20000 \\
\hline & & $\mathrm{IU} / \mathrm{L}$ & $\mathrm{IU} / \mathrm{L}$ & $\mathrm{IU} / \mathrm{L}$ \\
\hline \multirow[t]{2}{*}{ Acetaminophen SR } & 1 & 61.34 & 55.84 & 84.06 \\
\hline & 3 & 68.85 & 66.04 & 92.72 \\
\hline \multirow[t]{2}{*}{ Tramadol SR } & 1 & 59.77 & 46.86 & 61.62 \\
\hline & 3 & 64.72 & 58.63 & 78.21 \\
\hline
\end{tabular}

The high calculated $f_{2}$ values show that SD HASCA formulations performs well in the presence of $\alpha$-amylase, at least for the range of $\alpha$-amylase concentrations tested. It has been reported that the access to the $\alpha$-amylase active site involved in the amylolytic process is limited by the presence of the carboxymethyl groups, probably via a steric and/or an ionic interaction of carboxymethyl groups with the active site [20]. Consequently, the presence of the carboxymethyl groups of SD HASCA may have enhanced the resistance of the polymer to $\alpha$-amylase-catalyzed hydrolysis. Another explanations for the similarity between the release profiles may be given by the transformation of starch into a more crystalline form during hydrolysis [21], which is less easily degraded by the enzyme [22-24], and/or the inhibition of the enzymatic degradation by reaction by-products, such as maltose and maltotriose, which can reduce the rate of starch hydrolysis as the reaction progresses [22-25]. In addition, some 
studies have described a facilitated enzyme access to the site of amylolysis for high-amylose carboxymethyl starches produced with a higher DS due to their high hydrophilicity. The substitution of the starch hydroxyl groups with the highly hydrophilic carboxymethyl sodium groups induces an increase of the hydrophilicity and relative solubility of this modified starch, even in cold water, and leads to a higher drug-release rate $[20,26]$. A higher affinity of $\alpha-$ amylase (Bacillus species) for a soluble substrate than for a solid one has also been reported [25]. Since SD HASCA was produced with low DS, it possesses sufficient carboxymethyl groups to convey adequate resistance to $\alpha$-amylase but not enough groups to promote intense $\alpha$-amylase-catalyzed-hydrolysis caused by very high hydrophilicity. In addition, the higher the enzyme concentration was, the more significant the contribution of enzyme-catalyzed erosion was.

Although acetaminophen is less soluble than tramadol hydrochloride, Acetaminophen SR contained a high quantity of soluble materials $(240 \mathrm{mg}$ of acetaminophen and $165 \mathrm{mg}$ of sodium chloride) and a lower quantity of SD HASCA polymer than Tramadol SR. Nevertheless, the release profiles from Acetaminophen SR and Tramadol SR were similar, especially for the first half of the release, as indicated by the close values of T25\% and T50\% for all enzyme concentrations and both residence times in SGF, shown in Figures 5.1 (A) - 5.2 (B)), and by $f_{2}$ values between 66.48 and 78.96. The similarity between the release profiles from the two tested SD HASCA formulations showed that the model drug used and the other excipient present in the formulation did not significantly affect $\alpha$-amylase-catalyzed-erosion and, consequently, drug-release.

These results showed that the model drug used, the quantity of polymer and the type of excipients in the formulation did not significantly affect $\alpha$-amylase-catalyzed-erosion and, thus, drug-release.

5.5.1.2 Residence time in SGF does not significantly affect the drug-release profiles

As a result of the effect of food on gastric emptying, the gastric residence time of any non-disintegrating oral dosage form varies depending on whether it was administrated in the 
fasted or fed state. The unpredictable gastric residence time can influence drug-release from SR dosage forms and lead to variable drug bioavailability. Therefore, it was important to study the effect of varying the exposure time to SGF on drug-release from SD HASCA tablets in the presence of $\alpha$-amylase.

Table 5.1 shows that the $f_{2}$ values between the release profiles at different $\alpha$-amylase concentrations were slightly lower when the tablets were exposed to SGF for $1 \mathrm{~h}$ compared to $3 \mathrm{~h}$, for both formulations. Moreover, Table 5.2 shows that the $f_{2}$ values between the release profiles when the residence time in SGF was $1 \mathrm{~h}$ versus $3 \mathrm{~h}$ decreased as the concentration of enzyme increased, also for both formulations. An interpretation of these results can be given by the fact that when the tablets were exposed to SGF for $1 \mathrm{~h}$, the acidic buffer penetrated less deeply into their core, creating a narrower gel layer with an acidic environment. Thus, the SIF I with $\alpha$-amylase had to traverse a shorter acidic pathway when diffusing through the gel layer. Besides, $\alpha$-amylase is inactive in low $\mathrm{pH}$, and thus a narrower acidic gel layer possibly resulted in inferior enzyme inactivation. These phenomena resulted in a little more important contribution of $\alpha$-amylase-catalyzed erosion to the overall drug-release rate when the formulations were immersed in SGF for $1 \mathrm{~h}$, which led to a slightly higher release rate. The values of $\mathrm{T} 25 \%, \mathrm{~T} 50 \%$ and $\mathrm{T} 90 \%$ for Acetaminophen SR and Tramadol SR, seen in the Figures 5.1 (A) - 5.2 (B), also suggest a very slightly increase in the release rate when the residence time in SGF was $1 \mathrm{~h}$ and $\alpha$-amylase was present in SIF I, especially noticeable for the values of T50\%. However, as described above, these differences in drug-release rates were not significant.

A previous publication demonstrated that the in vitro release of acetaminophen from Acetaminophen SR was not influenced by variations of both the acidic $\mathrm{pH}$ value and residence time in the acidic medium, which simulated various possibilities of gastric $\mathrm{pHs}$ and residence times in the fasted and fed states [5]. The $f_{2}$ value of 91.02 (Table 5.2) found in the present study between the release profiles from Acetaminophen SR exposed to SGF for $1 \mathrm{~h}$ and $3 \mathrm{~h}$ at $0 \mathrm{IU} / 1$ of enzyme, and the relative proximity of the values of the $\mathrm{T} 25 \%$, T50\% and T90\% for the same release profiles (Figs. 5.1 (A) and 5.1 (B)) were in agreement with the results of that study. These findings are important because formulations that possess $\mathrm{pH}$-independent release 
properties are preferable for oral SR delivery, given that they are less likely to be affected by intra- and inter-subject variations of both gastric $\mathrm{pH}$ and gastric emptying time.

Table 5.2 Similarity factors $\left(f_{2}\right)$ between the release profiles from Acetaminophen SR and Tramadol SR at different residence times in simulated gastric fluid (pH 1.2).

\begin{tabular}{lll}
\hline Formulation & $\begin{array}{l}\alpha \text {-amylase } \\
\text { concentration } \\
(\text { IU/L })\end{array}$ & $\begin{array}{l}f_{2} \text { between release profiles at different residence times in SGF } \\
(\mathrm{pH} 1.2)\end{array}$ \\
\cline { 3 - 3 } & & 1 vs. 3 h \\
\hline Acetaminophen SR & 0 & 91.02 \\
& 5000 & 75.75 \\
Tramadol SR & 20000 & 65.14 \\
& 0 & 81.61 \\
& 5000 & 84.32 \\
& 20000 & 60.45 \\
\hline
\end{tabular}

\subsubsection{Contribution of $\alpha$-amylase-catalyzed erosion to the overall drug- release mechanism}

The determined $k_{2} / k_{1}$ ratios (Table 5.3) show that diffusion was the predominant drugrelease mechanism in most cases. The only exception was when Tramadol SR tablets were exposed to SGF for $1 \mathrm{~h}$ and to SIF I with $20000 \mathrm{IU} / \mathrm{L}$ of $\alpha$-amylase, since $k_{2} / k_{1}$ ratio was superior to 1, and thus erosion was the predominant mechanism. In the case of Acetaminophen $\mathrm{SR}$ in the same conditions the contribution of diffusion and erosion were equivalent, i.e., $k_{2} / k_{1}$ was equal to 1 . The contribution of the erosion mechanism to the overall drug-release rate increased as the concentration of enzyme increased for both formulations and both residence times in SGF, except for Acetaminophen SR tablets exposed to SGF for $3 \mathrm{~h}$ and to SIF I with $20000 \mathrm{IU} / \mathrm{L}$ of $\alpha$-amylase. Contrary to what was expected, in this case the $k_{2} / k_{1}$ ratio at 20000 $\mathrm{IU} / \mathrm{L}$ was equal to the ratio at $5000 \mathrm{IU} / \mathrm{L}$, which is in agreement with the small difference 
observed between the release profiles. The $k_{2} / k_{1}$ ratios also demonstrate that the contribution of the erosion mechanism was indeed more significant when the tablets were immersed in SGF for $1 \mathrm{~h}$, especially in the presence of enzyme and in the case of Tramadol SR.

Table 5.3 Values of $2 a / l$, Aspect Ratio (where $2 a$ is the diameter and $l$ is the thickness of the tablet); $m$, the estimated Fickian diffusion exponent; $k_{2} / k_{1}$, the erosion and diffusion kinetic constants ratio; and $R^{2}$, the correlation coefficient, for Acetaminophen SR and Tramadol SR, the two residence times in the acidic medium and the three $\alpha$-amylase concentrations tested.

\begin{tabular}{|c|c|c|c|c|c|c|}
\hline Formulation & $\begin{array}{l}\text { Residence time } \\
\text { in SGF (pH 1.2) }\end{array}$ & $\begin{array}{l}\alpha \text {-amylase } \\
\text { concentration } \\
(\mathrm{IU} / \mathrm{L})\end{array}$ & $2 a / l$ & $m$ & $k_{2} / k_{1}$ & $R^{2}$ \\
\hline \multirow{6}{*}{$\begin{array}{l}\text { Acetaminophen } \\
\text { SR }\end{array}$} & \multirow{3}{*}{1} & 0 & 4.040 & 0.45 & 0.47 & 0.9993 \\
\hline & & 5000 & & 0.45 & 0.71 & 0.9995 \\
\hline & & 20000 & & 0.45 & 1.01 & 0.9996 \\
\hline & \multirow{3}{*}{3} & 0 & 4.040 & 0.45 & 0.32 & 0.9995 \\
\hline & & 5000 & & 0.45 & 0.45 & 0.9997 \\
\hline & & 20000 & & 0.45 & 0.45 & 0.9999 \\
\hline \multirow{6}{*}{ Tramadol SR } & \multirow{3}{*}{1} & 0 & 1.499 & 0.43 & 0.15 & 0.9995 \\
\hline & & 5000 & & 0.43 & 0.65 & 0.9994 \\
\hline & & 20000 & & 0.43 & 1.79 & 0.9994 \\
\hline & \multirow{3}{*}{3} & 0 & 1.499 & 0.43 & 0.22 & 0.9995 \\
\hline & & 5000 & & 0.43 & 0.56 & 0.9988 \\
\hline & & 20000 & & 0.43 & 0.89 & 0.9975 \\
\hline
\end{tabular}

These results support the assumptions described above that a narrower acidic gel layer formed during $1 \mathrm{~h}$ of exposure to the acidic buffer and that a higher quantity of polymer favored $\alpha$-amylase action. For the same reason, the lower quantity of SD HASCA present in Acetaminophen SR and the larger acidic gel layer formed during $3 \mathrm{~h}$ of exposure to SGF explain why increasing $\alpha$-amylase concentration from $5000 \mathrm{IU} / \mathrm{L}$ to $20000 \mathrm{IU} / \mathrm{L}$ did not result 
in superior erosion in this case. The lowest value of $R^{2}$ was 0.9975 and, therefore, the quality of the least squares fitting of the Eq. 3 to the set of data points was excellent.

At $0 \mathrm{IU} / \mathrm{L}$ of $\alpha$-amylase in the SIF I, drug-release from SD HASCA tablets is controlled by a combination of drug diffusion through the gel layer and erosion at the surface of the matrix caused by the predominance of interactions water-polymer over interactions polymer-polymer [4]. The extent of the contribution of erosion to the overall drug-release rate can be negligible or become more important as the concentration of polymer in the tablet, and thus the gel-strength, decreases, as demonstrated in this study by a superior $k_{2} / k_{1}$ ratio for Acetaminophen SR at $0 \mathrm{IU} / \mathrm{L}$ of enzyme, as well as in a previous study [4]. On the other hand, in the presence of $\alpha$-amylase, erosion of the matrix due to the hydrolysis of $\alpha(1 \rightarrow 4)$ glycosidic bonds in the gel layer by $\alpha$-amylase plays a crucial role in the control of drugrelease, being that role more significant as the enzyme concentration increases.

Figure 5.3 illustrates the influence of $\alpha$-amylase concentration on the drug-release rate in function of release-time from Acetaminophen SR tablets immersed in SGF for $1 \mathrm{~h}$. When there was no $\alpha$-amylase in the medium, the drug-release profile can be divided into two stages. First, a burst effect occurred, which corresponded to the fast drug dissolution and release from the tablet surface before the gel layer was fully formed. Second, the drug-release rate decreased continuously until the end of the dissolution process. This decrease was due to the growth of the diffusion pathway that drug molecules have to traverse, as a result of the gel barrier that progressively forms as the medium penetrates into the core of the tablet. This release behavior is characteristic of a mainly diffusion-controlled mechanism [27]. When $\alpha-$ amylase was added to the SIF I, a minor increase in the drug-release rate was observed for the $3 \mathrm{~h}$ period during which the tablets were under the influence of $\alpha$-amylase. As expected, that rise was more important for a higher enzyme concentration. Figure 5.3 also suggests that the enzymes that penetrated deep into the matrices while the tablets were exposed to SIF I with $\alpha$ amylase kept their activity for about one to two hours after they were transferred to the SIF II with no enzymes, which happened $4 \mathrm{~h}$ after the beginning of drug-release. 


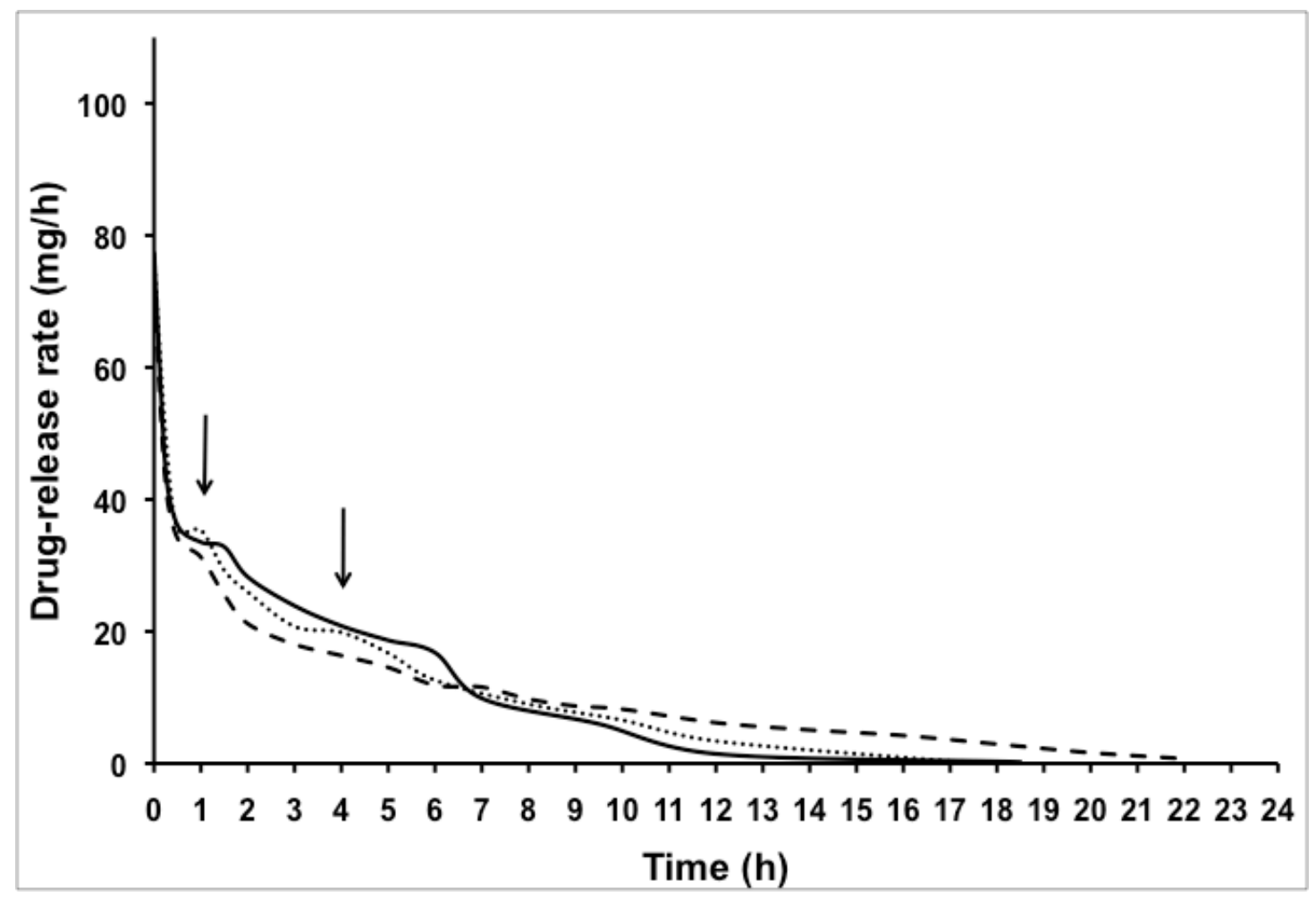

Figure 5.3 Acetaminophen-release rate from Acetaminophen SR tablets exposed $1 \mathrm{~h}$ to simulated gastric fluid $(\mathrm{pH} \mathrm{1.2)}$, and $3 \mathrm{~h}$ to simulated intestinal fluid $(\mathrm{pH} \mathrm{6.8)}$ with 0 IU/L (dashed line), $5000 \mathrm{IU} / \mathrm{L}$ (dotted line) and $20000 \mathrm{IU} / \mathrm{L}$ (continuous line) of $\alpha$-amylase. The arrows represent the time at which the tablets were placed $(1 \mathrm{~h})$ and were withdrawn $(4 \mathrm{~h})$ from the medium with $\alpha$-amylase.

Table 5.4 shows that drug-release from Tramadol SR was predominantly controlled by diffusion when there was no $\alpha$-amylase in the medium, since $R / F$ remained below 1 for all time points. In the case of drug-release from Acetaminophen SR, the contribution of erosion became significant $5.5 \mathrm{~h}$ or $12.5 \mathrm{~h}$ after the beginning of the release process, when the tablets were immersed $1 \mathrm{~h}$ or $3 \mathrm{~h}$ in SGF, respectively. However, the presence of $\alpha$-amylase in the medium seemed to have a greater impact on Tramadol SR, since the contribution of erosion for drug-release became significant quicker, especially for the higher enzyme concentration. 
Table 5.4 Time at which R/F became equal or superior to 1 , for the residence times in the simulated gastric fluid ( $\mathrm{pH} 1.2)$ and the $\alpha$-amylase concentrations tested.

\begin{tabular}{|c|c|c|c|}
\hline Formulation & Time in $\mathrm{pH} 1.2(\mathrm{~h})$ & $\begin{array}{l}\alpha \text {-amylase } \\
\text { concentration }(\mathrm{IU} / \mathrm{L})\end{array}$ & Time when $R / F \geq 1(h)$ \\
\hline \multirow[t]{6}{*}{ Acetaminophen SR } & 1 & 0 & 5.5 \\
\hline & & 5000 & 2 \\
\hline & & 20000 & 1 \\
\hline & 3 & 0 & 12.5 \\
\hline & & 5000 & 6 \\
\hline & & 20000 & 6 \\
\hline \multirow[t]{6}{*}{ Tramadol SR } & 1 & 0 & - \\
\hline & & 5000 & 3 \\
\hline & & 20000 & 0.25 \\
\hline & 3 & 0 & - \\
\hline & & 5000 & 3.8 \\
\hline & & 20000 & 1.3 \\
\hline
\end{tabular}

As explained before, at $0 \mathrm{IU} / \mathrm{L}$ of $\alpha$-amylase, the much larger quantity of SD HASCA present in Tramadol SR compared to Acetaminophen SR enhanced the resistance of the first formulation to erosion because of the strength of the hydrophilic gel formed, resulting in diffusion of drug molecules through the gel layer being the predominant drug-transport mechanism during the entire drug-release process. In contrast, the lower quantity of polymer and the high quantity of soluble materials present in Acetaminophen SR may have led to the creation of a more open porous network during the dissolution process, increasing the importance of the erosion mechanism in the absence of enzyme at a certain point, which happened earlier when the residence time in SGF was $1 \mathrm{~h}$ for the causes already described. On the other hand, when $\alpha$-amylase was present in SIF I, the higher content in wettable SD HASCA and the high solubility of tramadol hydrochloride of Tramadol SR, promoted $\alpha-$ 
amylase penetration and the susceptibility of this formulation to enzyme-catalyzed erosion. The higher the enzyme concentration was, the more significant the $R / F$ ratio was.

It could be expected that the addition of an electrolyte to Acetaminophen SR would also propel the fast penetration of SIF I with enzymes inside the tablet. However, a previous study has shown that this is not the case [5]. This phenomenon can be explained by the Donnan equilibrium [28]. According to this theory, in a gel network with immobile negative charge, in this case from the ionized carboxymethyl groups, the presence of solubilized sodium mobile counterions inside the gel network, resulting from the dissociation in alkaline medium, may reduce the osmotic pressure and, as a consequence, the penetration of medium into the tablets.

\subsection{Conclusions}

The present study shows that $\alpha$-amylase does not significantly affect the drug-release profiles from two different SD HASCA formulations, regardless of the residence time in acidic medium. These findings support the results of a clinical study, where Acetaminophen SR was administered to healthy human subjects, which showed that the tablets released the drug over an extended period of time and resisted $\alpha$-amylase biodegradation while transiting the gastrointestinal tract. The application of a two-component model that allows the investigation of the contribution of the diffusion and the erosion mechanisms to the overall drug-release rate shows that depending on the tablet composition and $\alpha$-amylase concentration, the mechanism controlling drug-release from SD HASCA tablets can switch from drug diffusion to polymer erosion. These results support the potential of SD HASCA as a promising excipient for oral SR matrix tablets, since they show that the presence of $\alpha$-amylase in the gastrointestinal tract and variations of the gastric emptying of SD HASCA tablets do not have a significant impact on their drug-release properties, at least for the residence times in acidic medium and the $\alpha$-amylase concentrations tested. 


\subsection{Acknowledgements}

This paper is dedicated to the living memory of Prof. Louis Cartilier. Teresa Nabais gratefully acknowledges the scholarship support of Fundação para a Ciência e Tecnologia (Portugal). Also, thank you Jeanne Leblond Chain and all the students and members of the Group Meeting Axe Formulation for proofreading this paper and for your helpful suggestions.

\subsection{References}

[1] J.M. Fang, P.A. Fowler, C. Sayers, P.A. Williams, The chemical modification of a range of starches under aqueous reaction conditions, Carbohydrate Polymers, 55 (2004) 283-289.

[2] E. Svensson, A.-C. Eliasson, Crystalline changes in native wheat and potato starches at intermediate water levels during gelatinization, Carbohydrate Polymers, 26 (1995) 171-176.

[3] A. Rajan, V.S. Prasad, T. Emilia Abraham, Enzymatic esterification of starch using recovered coconut oil, International Journal of Biological Macromolecules, 39 (2006) 265272.

[4] F. Brouillet, B. Bataille, L. Cartilier, High-amylose sodium carboxymethyl starch matrices for oral, sustained drug-release: Formulation aspects and in vitro drug-release evaluation, International journal of pharmaceutics, 356 (2008) 52-60.

[5] T. Nabais, F. Brouillet, S. Kyriacos, M. Mroueh, P. Amores da Silva, B. Bataille, C. Chebli, L. Cartilier, High-amylose carboxymethyl starch matrices for oral sustained drugrelease: In vitro and in vivo evaluation, European Journal of Pharmaceutics and Biopharmaceutics, 65 (2007) 371-378.

[6] L. Cartilier, M. Ungur, C. Chebli, Tablet Formulation for Sustained Drug-release, Canadian Patent Application Canada No 2491665 (2005).

[7] M. Ungur, N. Yonis, C. Chebli, L. Cartilier, The evaluation of carboxymethylamylose for oral drug delivery systems: from laboratory to pilot scale, in: Books of Abstracts of the ISAB2-2005 3rd International Symposium on Advanced Biomaterials/Biomechanics, Montreal, Canada, 2005, pp. 271. 
[8] F. Brouillet, G. Baylac, L. Cartilier, B. Bataille, High-amylose sodium carboxymethyl starch matrices for oral, sustained drug release: development of a spray drying manufacturing process, Drug development and industrial pharmacy, 36 (2010) 795-805.

[9] Q.R. Cao, Y.W. Choi, J.H. Cui, B.J. Lee, Formulation, release characteristics and bioavailability of novel monolithic hydroxypropylmethylcellulose matrix tablets containing acetaminophen, J Control Release, 108 (2005) 351-361.

[10] S. Grond, A. Sablotzki, Clinical pharmacology of tramadol, Clin Pharmacokinet, 43 (2004) 879-923.

[11] T. Nabais, G. Leclair, High-amylose carboxymethyl starch matrices: development and characterization of tramadol hydrochloride sustained-release tablets for oral administration (Unpublished results).

[12] G. Ferey-Roux, J. Perrier, E. Forest, G. Marchis-Mouren, A. Puigserver, M. Santimone, The human pancreatic alpha-amylase isoforms: isolation, structural studies and kinetics of inhibition by acarbose, Biochim Biophys Acta, 14 (1998) 10-20.

[13] D. Clem, J. Maidment, J.M. Ringham, A study into the measurement of alpha-amylase activity using phadebas, iodine and gel-diffusion procedures, Nutrition and food science, 31 (2001) 141-146.

[14] C. Chebli, L. Cartilier, N.G. Hartman, Substituted amylose as a matrix for sustained-drug release: a biodegradation study, International journal of pharmaceutics, 222 (2001) 183-189.

[15] M. Rahmouni, F. Chouinard, F. Nekka, V. Lenaerts, J.C. Leroux, Enzymatic degradation of cross-linked high amylose starch tablets and its effect on in vitro release of sodium diclofenac, Eur J Pharm Biopharm, 51 (2001) 191-198.

[16] Y. Dumoulin, L.H. Cartilier, M.A. Mateescu, Cross-linked amylose tablets containing $\alpha-$ amylase: an enzymatically-controlled drug release system, Journal of Controlled Release, 60 (1999) 161-167.

[17] USP 36 - NF 31, (United States Pharmacopeia 36 - National Formulary 31), U.S Pharmacopeial Convention, Rockville, MD, 2013.

[18] J.W. Moore, H.H. Flanner, Mathematical comparison of dissolution profiles, Pharm. Tech., 20 (1996) 64-74. 
[19] N.A. Peppas, J.J. Sahlin, A simple equation for the description of solute release. III. Coupling of diffusion and relaxation, International journal of pharmaceutics, 57 (1989) 169172.

[20] C. Calinescu, J. Mulhbacher, É. Nadeau, J.M. Fairbrother, M.A. Mateescu, Carboxymethyl high amylose starch (CM-HAS) as excipient for Escherichia coli oral formulations, European Journal of Pharmaceutics and Biopharmaceutics, 60 (2005) 53-60.

[21] P. Colonna, A. Buleon, F. Lemarie, Action of Bacillus subtilis alpha-amylase on native wheat starch, Biotechnol Bioeng, 31 (1988) 895-904.

[22] D.J. Gallant, B. Bouchet, A. Buleon, S. Perez, Physical characteristics of starch granules and susceptibility to enzymatic degradation, Eur J Clin Nutr, 46 (1992) S3-16.

[23] V.M. Leloup, P. Colonna, S.G. Ring, Physico - chemical aspects of resistant starch, Journal of Cereal Science, 16 (1992) 253-266.

[24] V. Planchot, P. Colonna, D.J. Gallant, B. Bouchet, Extensive degradation of native starch granules by alpha-amylase from aspergillus fumigatus, Journal of Cereal Science, 21 (1995) 163-171.

[25] V.M. Leloup, P. Colonna, S.G. Ring, alpha-Amylase adsorption on starch crystallites, Biotechnol Bioeng, 38 (1991) 127-134.

[26] M. Lemieux, P. Gosselin, M.A. Mateescu, Carboxymethyl high amylose starch as excipient for controlled drug release: Mechanistic study and the influence of degree of substitution, International journal of pharmaceutics, 382 (2009) 172-182.

[27] Y.W. Chien, Fundamentals of controlled drug administration, in: J. Swarbrick (Ed.) Novel Drug Delivery System, Marcel Dekker Inc., New York, 1982, pp. 465-574.

[28] A.R. Khare, N.A. Peppas, Swelling/deswelling of anionic copolymer gels, Biomaterials, 16 (1995) 559-567. 


\section{Chapter 6. General discussion, conclusions and perspectives}

\subsection{General discussion}

The main objective of this research was to further demonstrate the qualities of SD HASCA for future use as a safe and efficient excipient in the production of oral SR dosage forms, and thus provide additional arguments for the industrial development of this excipient. For this purpose, dissolution tests that simulated physiological conditions that may influence the performance of SD HASCA dosage forms in the gastrointestinal tract and an exploratory clinical study were carried out. In addition, two twice-daily and two once-daily SD HASCA formulations containing doses of tramadol hydrochloride commonly administered in SR dosage forms were developed. Further clinical studies, pharmacokinetic evaluations and the development of IVIVC involving these four formulations and a greater number of volunteers were part of the initial plan of this research. However, it was not possible to perform these studies.

\subsubsection{Advantages of SD HASCA}

Glycidol, the substituent used to produce the first generation of SA polymers with high-amylose starch (SA,G- $n$ ), has shown potential toxicity, including evidence of carcinogenic activity (US Department of Health and Human Services (2007)). Therefore, further in vitro dissolution experiments (Cartilier, Ungur et al. 2005; Ungur, Yonis et al. 2005) were carried out to validate a substitution of the substituent in order to produce a safer SA polymer, and thus facilitate the acceptance of the excipient by the regulatory agencies. The new substituent was sodium chloroacetate, which has already been applied in the production of a well-know disintegrant agent, i.e., sodium starch glycolate, a sodium carboxymethyl starch containing low amylose content (e.g., Explotab ${ }^{\circledR}$ or Glycolys ${ }^{\circledR}$ ) (Edge and Miller 2005). The improved safety and consequent easier approval of HASCA represent an important 
advantage of this new excipient. Subsequently, a process to produce the new excipient using spray drying was designed in order to prepare the scale-up and promote easier and economical industrial production of SD HASCA (Brouillet, Baylac et al. 2010).

SD HASCA matrix tablets are produced by direct compression, which is the easiest way to manufacture an oral dosage form. Besides, due to the excellent binding properties of SD HASCA there is no need to add a binder to the formulation. All formulations developed in this study presented high crushing strength values, independently of the model drug chosen, the presence of sodium chloride in the formulation, the concentrations of each of the ingredients in the tablet and the TW. This feature may potentially provide the means for simplifying formulation development and improving overall operational costs and time while preserving the quality of the product, since a single excipient is the solution for more than one feature. Moreover, these high crushing strengths reduce the likelihood of accidental misuse by the patients, such as breaking or crushing of tablets.

Two hypotheses were put forward to explain these good binding properties of SD HASCA. First, as the HASCA particles did not possess any binding properties before spray drying and this procedure resulted in a reduction of the particle size of the polymer, the particle size reduction and the consequent increase of the surface area of the particulate product may provide higher number of binding points. Secondly, it was suggested that the combination of water and ethanol in the spray-drying procedure has a plasticizer effect, leading to melting of the polymer under compression, which intensified the densification of the matrices (Brouillet, Baylac et al. 2010).

The lack of influence of the CF applied (within a range normally employed at the industrial level) to produce the tablets on the release profiles of tramadol hydrochloride from SD HASCA matrices (containing $14.3 \%$ of drug and $85.7 \%$ of polymer) indicated that the matrix porosity, if any, was very low and had no significant effect on drug-release. The same independence between $\mathrm{CF}$ and drug-release was also observed in a previous study (Brouillet, Bataille et al. 2008) for tablets containing SD HASCA with $70 \%$ of amylose instead of $60 \%$ and spray-dried with a slightly different final ethanol/HASCA ratio, acetaminophen as the model drug (40\%), and sodium chloride (27.5\%). These results show that the independence 
between drug-release and the applied CF was not affected by the different composition of the polymer, the final ethanol/HASCA ratio, the substitution of sodium chloride for tramadol hydrochloride as the electrolyte in the formulation and the model drug used, which emphasizes the versatility of this new polymer as an excipient for the production of SR dosage forms.

The network formed by the SD HASCA chains when in contact with an aqueous medium was strong enough to permit the matrices to keep their SR properties, even in the presence of high loadings of soluble drugs such as tramadol hydrochloride. This strong gel is also important because the mechanical strength of a SR dosage form and its capacity to resist the powerful peristaltic contractions occurring in the gastrointestinal tract in the fasted state is an essential requisite in the development of this type of dosage forms. A weak mechanical strength may lead to premature drug-release and, therefore, to the failure of the formulation with respect to its ability to extend drug-release.

Dissolution tests comparing the release profiles of a SD HASCA formulation and a HPMC formulation containing the same percentages of acetaminophen, sodium chloride and polymer showed that the drug-release from the HPMC formulation was slightly slower (Figure 6.1). However, the visual observation of the matrices during the dissolution test revealed that the degree of swelling of the gel formed by SD HASCA was moderate when compared to HPMC, a hydrophilic polymer commonly used in SR. In addition, the mechanical resistance of the gel formed by SD HASCA seemed to be superior compared the to gel formed by HPMC because HPMC showed more gradual polymer erosion during the dissolution tests. However, studies on the degree of erosion were not performed. This mechanical strength may represent an advantage of the SD HASCA polymer for SR applications for the reasons already described. 


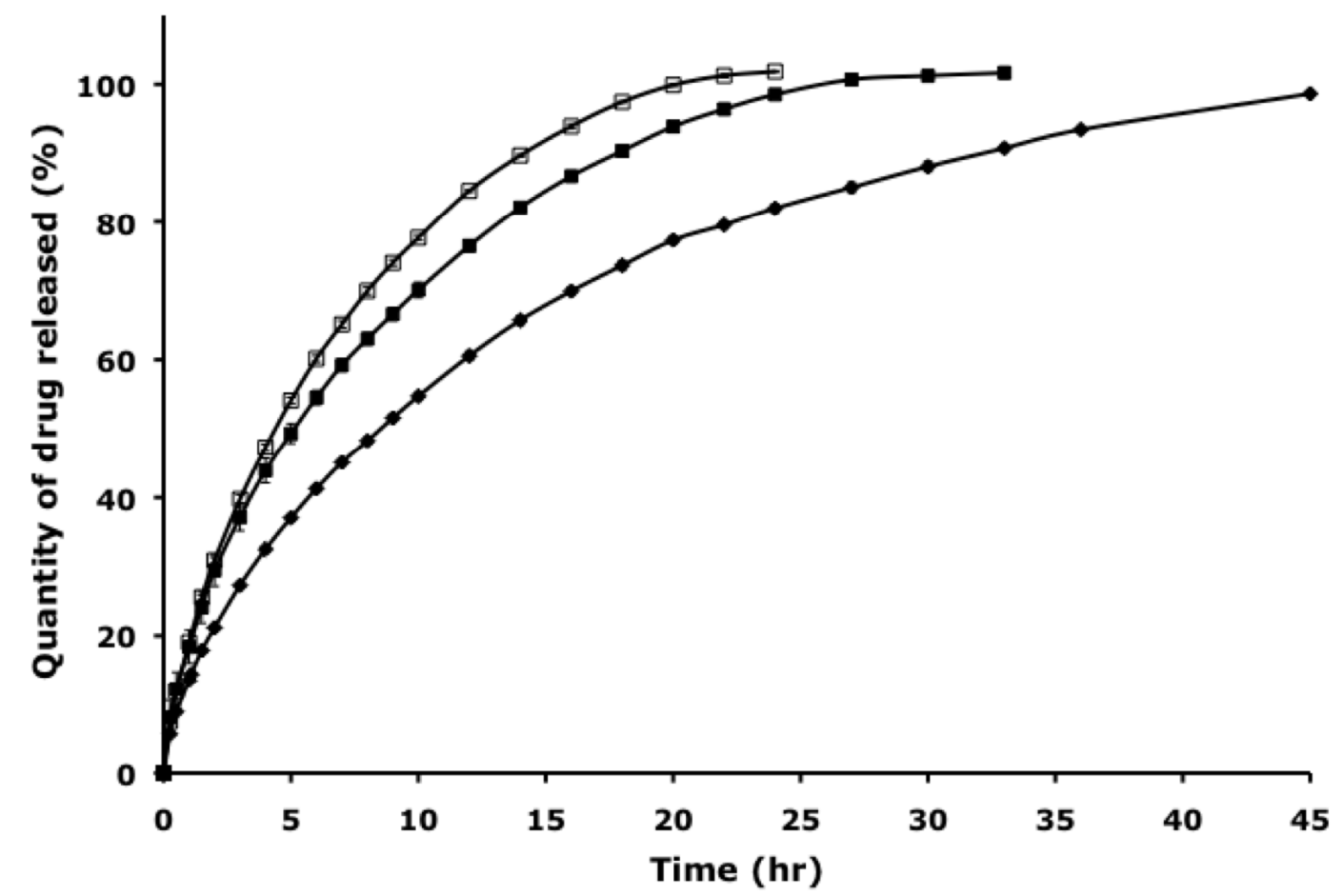

Figure 6.1 Cumulative percent of acetaminophen released in vitro from $\square$ : SD HASCA matrices containing $40 \%$ of acetaminophen, $27.5 \%$ of $\mathrm{NaCl}$ and $32.5 \%$ of polymer; $\mathbf{\square}$ : HPC matrices containing $40 \%$ of acetaminophen, $27.5 \%$ of $\mathrm{NaCl}$ and $32.5 \%$ of polymer; and from $\checkmark$ : HPMC matrices containing $40 \%$ of acetaminophen, $0 \%$ of $\mathrm{NaCl}$ and $60 \%$ of polymer. The number of replicates was three.

\subsubsection{Influence of $\mathrm{pH}$, gastric residence time, $\alpha$-amylase and an alcoholic medium on the drug-release from SD HASCA matrices}

Because of the prolonged residence time of SD HASCA tablets in the gastrointestinal tract, their in vivo performance is more susceptible to the environmental factors these formulations will encounter when transiting along the digestive system. As described in the section 1.7 of this thesis, these variables are very complex and difficult to simulate, and no dissolution test can reproduce precisely the in vivo release environment. However, the development of dissolution test methods for new SR dosage forms should involve an 
assessment of at least some of the physiological conditions the dosage form will come across in the lumen of the gastrointestinal tract. Thereby, some of these gastrointestinal physiological conditions were simulated in vitro in this research.

First, the in vitro performance of SD HASCA formulations was investigated using a series of dissolution media simulating the $\mathrm{pH}$ range along the gastrointestinal tract (SGF, SIF I and SIF II), the residence time in each $\mathrm{pH}$ and possible variations in the gastric $\mathrm{pH}$ and residence time caused by the ingestion of food. A SGF and residence time values in SGF simulating fasting conditions (10 min and $1 \mathrm{~h}$ in $\mathrm{pH} 1.2)$ as well as the effect of food on gastric $\mathrm{pH}$ and residence time $(3 \mathrm{~h}$ in $\mathrm{pH} 3.5$ and $6 \mathrm{~h}$ in $\mathrm{pH} 4.5)$ were employed in the case of the formulation containing acetaminophen in order to better foresee the effect of these physiological variants on the drug-release from SD HASCA matrices. These values were in accordance with values reported in the literature, although these physiological factors vary greatly. In addition, the drug-release profiles after 1 or $3 \mathrm{~h}$ in SGF, followed by SIF I without enzymes, were also compared for Acetaminophen SR and Tramadol SR. The drug-release profiles from SD HASCA tablets showed to be independent of both the SGF value and the residence time in SGF, regardless of the formulation and the model drug used. A possible explanation for this occurrence was given by the simultaneous presence of an electrolyte and the carboxylic groups of the polymer, creating a possible buffer environment in the matrix, which may have rendered the formulation insensitive to $\mathrm{pH}$ changes of the surrounding medium. The $\mathrm{pH}$-independence of the release kinetics of SD HASCA matrices represents an advantage of these formulations given that it greatly limits the influence of intra- and intersubject variability of these physiologic parameters on the release kinetics from SD HASCA matrices.

SD HASCA is a chemically modified starch product, and thus it is digested by salivary and pancreatic $\alpha$-amylases. The production of pancreatic $\alpha$-amylase is induced by food intake. Since SD HASCA tablets may contain amounts of drug that may lead to adverse effects if released too fast, it was important to investigate the influence of the hydrolysis of this starchbased excipient by $\alpha$-amylase on the rate and mechanisms of drug-release from SD HASCA 
formulations. This investigation involved both Acetaminophen SR and Tramadol SR. The effect of varying the exposure time to SGF on the $\alpha$-amylase-catalyzed erosion of these formulations was also evaluated because gastric residence time can be rather unpredictable as a result of the effect of food on gastric emptying.

The results of this part of the investigation showed that $\alpha$-amylase-catalyzed erosion of both formulations did not result in a significant increase in the drug-release rates, at least for the range of $\alpha$-amylase concentrations tested. Explanations found for this minor influence were given in part by the limited access to the $\alpha$-amylase active site caused by the presence of the carboxymethyl groups of SD HASCA (Calinescu, Mulhbacher et al. 2005). The transformation of starch into a less digestible form and/or inhibition of the enzymatic degradation by reaction by-products may also reduce the rate of starch hydrolysis as the reaction progresses (Leloup, Colonna et al. 1991; Gallant, Bouchet et al. 1992; Leloup, Colonna et al. 1992; Planchot, Colonna et al. 1995). In addition, high-amylose carboxymethyl starches produced with a higher DS have been associated with a more facilitated enzyme access to the site of amylolysis because of their higher hydrophilicity (Lemieux, Gosselin et al. 2009). SD HASCA has a low DS, and thus it may possess enough carboxymethyl groups to help hinder the access of the enzyme to the active site implied in the amylolytic process but not enough groups to promote intense $\alpha$-amylase-catalyzed-hydrolysis caused by very high hydrophilicity.

The drug-release profiles from Acetaminophen SR and Tramadol SR were similar for all enzyme concentrations and both residence times in SGF (except for a slight difference in one case) regardless of the different composition and polymer content of each formulation. These results showed that the model drug used, the quantity of polymer and the type of excipients in the formulation did not significantly affect $\alpha$-amylase-catalyzed-erosion and, thus, drug-release.

The results regarding the influence of the residence time in SGF on the $\alpha$-amylasecatalyzed erosion of SD HASCA tablets showed that the residence time did not significantly affect the enzyme activity and, consequently, the drug-release profiles from these formulations. There was, though, a slightly more important contribution of $\alpha$-amylasecatalyzed-erosion to the overall drug-release rate when the formulations were immersed in 
$\mathrm{SGF}$ for $1 \mathrm{~h}$ when compared to $3 \mathrm{~h}$. This effect increased as the enzyme concentration increased and led to a moderately higher drug-release rate. An explanation for this occurrence was given by the fact that after $1 \mathrm{~h}$ of exposure to SGF a narrower acidic gel layer was created. $\alpha$-Amylase becomes inactive in low $\mathrm{pH}$ and, therefore, a shorter acidic diffusion pathway may have resulted in inferior enzyme inactivation and in an earlier access of the SIF I with enzymes to the core of the tablet. However, the differences in the drug-release rates were not significant.

The resistance of the gel formed by SD HASCA to an alcoholic medium was assessed in order to assure the development of formulations that will withstand eventual coadministration with high doses of ethanol after oral administration and, thus, prevent the occurrence of undesirable outcomes. In 2005, the FDA warned of the possibility that the presence of alcohol in the gastrointestinal tract interacts with modified-release dosage forms, increasing the risk of alcohol-induced dose dumping, due to tablet rupture and erosion, and consequent accumulation in the body. A general regulatory approach to promote the design of modified-release dosage forms that are not sensitive to alcohol was outlined (Meyer and Hussain 2005). To achieve this goal, it is essential to evaluate the dose dumping potential in the early stages of product development so that only robust product designs are developed. Therefore, the importance of dissolution studies using a hydro-alcoholic medium.

The results of these studies showed that both SD HASCA and Contramid ${ }^{\circledR}$ (a crosslinked starch), the excipient of the commercial formulation used for comparison, formed a very viscous and strong gel in the hydro-alcoholic medium. The release rate from both formulations was significantly slower in the alcoholic environment than in a $\mathrm{pH}$ gradient, which suggests that it was more difficult for the drug to diffuse through the gel layer when the formulations were immersed in the hydro-alcoholic dissolution medium because of the higher strength of the gel. It was hypothesised that this decline in the drug-release rate was due to the eventual precipitation of amylose chains as Vh-amylose crystals. Further studies on the influence of ethanol on the solubility of SD HASCA are necessary to understand the decrease in release rate. Another explanation is a different solubility of tramadol hydrochloride in the $\mathrm{pH}$ gradient and in the ethanolic medium. 


\subsubsection{Factors influencing the gel layer formation and drug-release}

As described before, the formation of a gel layer around the dry core of the matrix in response to water penetration (Figure 6.2 a)) is the most important aspect of the release mechanism and kinetics from SR systems using hydrophilic polymers as the retarding excipient. This gel layer acts like a barrier against fast inward medium penetration and fast drug diffusion through and out of the matrix.

The dissolution profiles of a number of formulations with different total TW and different drug contents and containing only tramadol hydrochloride and SD HASCA were evaluated in a series of buffers simulating the $\mathrm{pH}$ throughout the gastrointestinal tract. As it is usually observed for hydrophilic matrices, the total release time from SD HASCA tablets increased significantly in function of the TW, and can thus be used to modulate the total release time from these formulations. A study suggested that the surface, thickness and structure of the gel layer may be nearly the same for each TW since the medium penetrates at the same rate to a certain depth of the tablet, regardless of its size (Varma, Kaushal et al. 2004). Therefore, if one considers the dry and/or partially hydrated core of the SD HASCA matrix as a drug reservoir (Figure $6.2 \mathrm{a}$ )), the increase in the total release time as a function of TW and size, may be, in part, explained by the larger size of this drug reservoir as the weight/size increases. However, studies using techniques such as image analysis should be performed to confirm this hypothesis.

In addition, as the TW decreases the formation of an external tight gel network capable of controlling water and drug diffusion becomes more difficult. Therefore, when tablets are very thin the matrix may become totally hydrated and gelled rapidly, likely resulting in a faster erosion of the matrix. Contrarily, increasing TW and TT heightens the contribution of the diffusion mechanism. This may explain why the tablets produced with a SSP machine led to slower drug-release rates, since they were thicker and slightly narrower. 

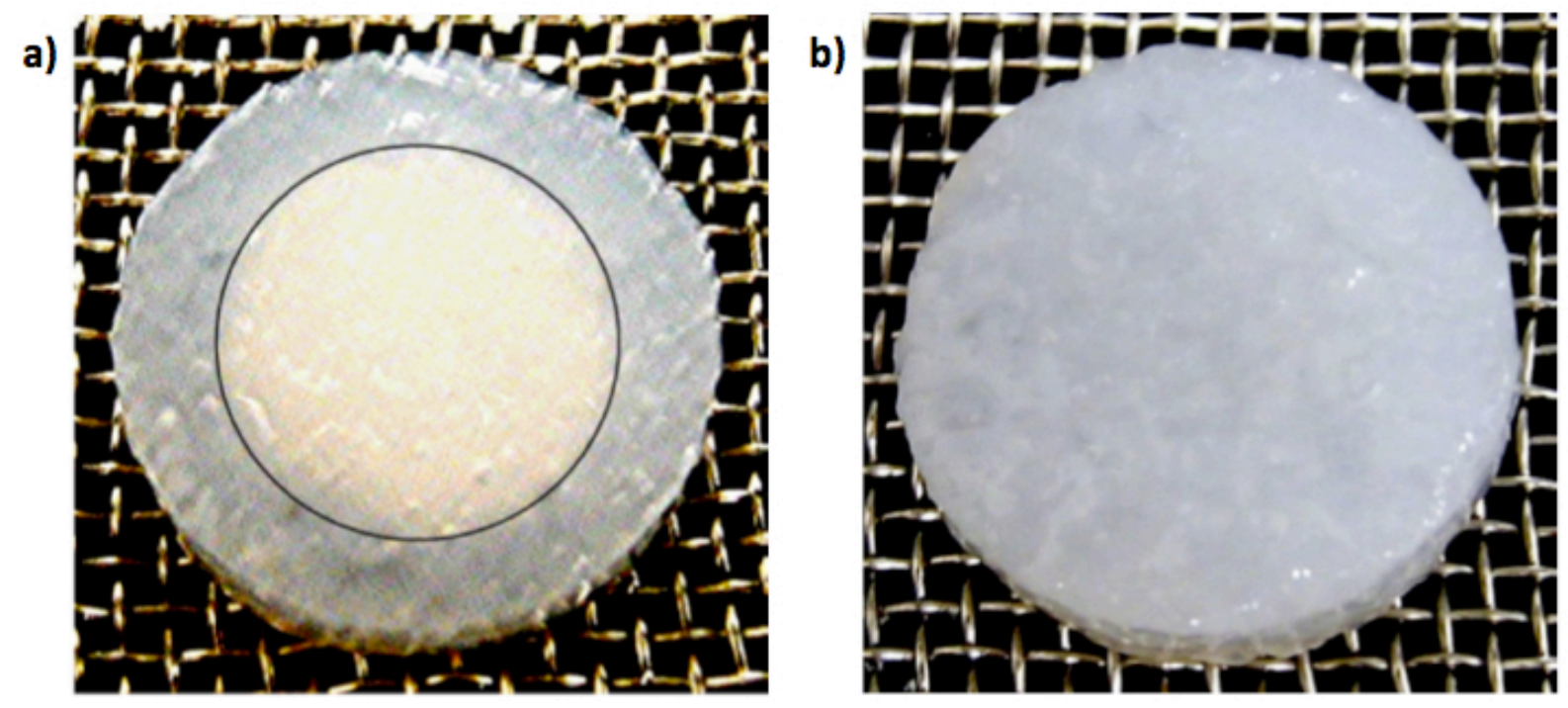

Figure 6.2 600-mg SD HASCA tablet matrices (40\% acetaminophen, $27.5 \% \mathrm{NaCl}, 32.5 \% \mathrm{SD}$ HASCA) after immersion in a $\mathrm{pH}$ gradient simulation the $\mathrm{pH}$ evolution of the gastrointestinal tract ( $\mathrm{pH} 1.2$ for $1 \mathrm{~h}, \mathrm{pH} 6.8$ for $3 \mathrm{~h}$, and $\mathrm{pH} 7.4$ until the end of the dissolution test): a) $16 \mathrm{~h}$ of immersion (gel layer formation), and b) $22 \mathrm{~h}$ of immersion (total hydration of the matrix). Figure reproduced from (Brouillet, Bataille et al. 2008) with permission of Elsevier Ltd.

This research also showed that another factor affecting the gel layer formation is the drug content and solubility. A more hydrophilic and, consequently, water soluble drug, like tramadol hydrochloride, together with a high drug content will increase the drug concentration in the gel and will promote the diffusion of the dissolution medium into the tablet. Above a certain threshold, the quantity of water may be such that the interactions between water and the polymer chains become superior to the interactions between polymer chains, resulting in progressive erosion of the gel layer. When polymer disentanglement is more rapid than water penetration the polymer gel layer will not grow and the drug-release rate will increase. 
In addition, the presence of $\alpha$-amylase in the medium seemed to hinder the continuous growth of the polymer gel layer due to erosion of the polymeric chains, and thus accelerates drug-release, being this effect higher as the concentration of enzyme rises.

An optimized amount of sodium chloride was added to the formulation containing acetaminophen to help maintain the integrity of the structure of the gel layer when the tablets were immersed in a $\mathrm{pH}$ gradient, as an increase in the drug-release rate was observed at the time of the appearance of cracks on the tablet surface. As tramadol hydrochloride is also an electrolyte, no extra electrolyte was added to the second type of formulations of this research. The small fissures that appear on the tablet surface of the formulations containing tramadol hydrochloride did not significantly affect the drug-release profiles. A possible explanation found for this occurrence was that the addition of an optimal amount of electrolyte may maintain an equilibrium between a) hydrogen bounds formed, among others, through the association of $-\mathrm{COOH}$ groups, which increase gel strength and help maintain matrix structure, and b) swelling of the polymer chains due to the repulsion between them caused by $-\mathrm{COO}^{-}$ groups, which provides the matrix with its necessary elasticity.

\subsubsection{In vivo studies and establishment of a Level A IVIVC}

An exploratory clinical study was performed in the early stages of development to assess the SR properties of a selected SD HASCA formulation containing acetaminophen in vivo. For this purpose, the pharmacokinetic parameters of the SD HASCA formulation were compared to those of a commercial IR formulation, after oral administration of these two formulations to healthy volunteers under fasting conditions. Furthermore, taking into account the importance of establishing a relevant correlation between in vitro drug-release and in vivo absorption in the early stages of the development of new dosage forms, a Level A IVIVC was attempted for the SD HASCA formulation. 
The comparison of the pharmacokinetic parameters estimated from the plasma concentration versus time curves resulting from the SD HASCA formulation and the commercial IR formulation demonstrated a prolonged absorption of acetaminophen after oral administration of SD HASCA matrix tablets. Also, it suggested that the total amounts of drug absorbed by the body (calculated after dose adjustment) were similar for the SR formulation and the IR formulation, irrespective of the absorption rate.

Furthermore, this study showed that there was no burst-effect while the tablets passed through the stomach and through the intestine. Therefore, the gel formed by SD HASCA was strong enough to resist the strong peristaltic contractions occurring in the fasted state as well as hydrolysis by $\alpha$-amylase in the intestine. These observations confirmed the hypothesis established while testing the formulations in vitro that it is possible to produce robust dosage forms using SD HASCA which do not show significant disintegration and/or hydrolysis throughout the gastrointestinal tract and which show $\mathrm{pH}$-independent release. However, the results of the in vivo study have to be interpreted cautiously because they were carried out using a limited number of volunteers.

The methodology used for the evaluation of IVIVC involved the in vitro drug-release characterization of a selected formulation, assessment of the hypothetical drug absorption profile using the Wagner-Nelson method and, finally, plotting of the percent of drug absorbed in vivo at specific time points against the percent dissolved in vitro at the same time points.

The relationship between the in vitro drug-release time course and the amount of drug absorbed from SD HASCA matrix tablets presented certain deviations from the ideal linear relationship and suggested a faster release rate in vivo. Some of the explanations for these results could be indeed a faster release in vivo or a possible high elimination rate of acetaminophen. It was, therefore, concluded that acetaminophen was not the most appropriate drug for this type of dosage form because of its high elimination rate, and that a new model drug with longer elimination $t_{1 / 2}$ compared to acetaminophen was needed to continue the development of SD HASCA as an excipient for SR. Since it is known that drugs with intermediate to short, but not very short $t_{1 / 2}$ values, provide the best candidates for SR products, the new drug chosen was tramadol hydrochloride, which has a $t_{1 / 2}$ between 5 and 7 hours. In addition, this clinical study also suggested that the Wagner-Nelson method was 
possibly not the most appropriate deconvolution method. A slightly deviation of the intercept value from zero was also observed, and was explained by a probable longer time for hydration of the tablets in vivo, leading to a delay in the begging of drug-release.

In conclusion, the first clinical study showed that new SD HASCA formulations with a new model drug, further dissolution studies evaluating these formulations and a new clinical study are required to continue the development of SD HASCA. An improved Level A IVIVC is necessary so that the set of dissolution conditions used to establish the correlation is able to provide a prediction of the in vivo behaviour of these formulations. A description of some of the dissolution experiments that can be performed to have a more accurate simulation of the conditions in the gastrointestinal tract, and thus an improved Level A IVIVC, are given in a following section.

\subsubsection{Mechanism controlling drug-release from SD HASCA matrices: Fickian diffusion versus relaxation/erosion}

It was hypothesized that Fickian diffusion of drug molecules through the gel layer is not the only transport mechanism controlling drug-release from SD HASCA matrices. Indeed, the results of this research showed that the polymer disentanglement and erosion while the matrix hydrates are also involved in the control of the kinetics of drug-release, even when there is no $\alpha$-amylase in the medium.

The determination of $k_{2} / k_{1}$ ratios (where $k_{1}$ is the Fickian kinetic constant and $k_{2}$ is the relaxational/erosion kinetic constant) permitted a better understanding and characterization of the mechanisms governing the release of drugs from matrix tablets produced with SD HASCA, either when these tablets were exposed to $\alpha$-amylase or when there was no enzyme in the medium. This ratio represents the contribution of the relaxation/erosion of chains compared to the contribution of Fickian diffusion. 
When $\alpha$-amylase was added to the SIF I, the determination of the $k_{2} / k_{1}$ ratios demonstrated that the contribution of erosion to the overall drug-release rate increased with the concentration of enzyme for both Acetaminophen SR and Tramadol SR and both residence times in SGF. The values of $k_{2} / k_{1}$ also confirmed a slightly more significant effect of erosion when the tablets were exposed $1 \mathrm{~h}$ to SGF and, therefore, sustained the hypothesis that a narrower acidic gel layer favoured $\alpha$-amylase action. In this case, erosion of the matrix caused by hydrolysis of $\alpha-(1 \rightarrow 4)$ glycosidic bonds in the gel layer by $\alpha$-amylase plays a role in the control of drug-release, increasing that role as the enzyme concentration increases. The higher content in hydrophilic polymer and the higher solubility of tramadol hydrochloride of Tramadol SR may have promoted the penetration of medium with enzyme and, therefore, the susceptibility of this formulation to enzyme-catalyzed-erosion relatively to Acetaminophen SR, especially for superior enzyme concentrations.

As described before, it was hypothesized that when there is no $\alpha$-amylase in the SIF I, drug-release from SD HASCA formulations is controlled mainly by diffusion but also by erosion on the surface of the matrices, which happens when the interactions water-polymer exceed the interactions polymer-polymer. Therefore, in this case, the contribution of erosion to the overall drug-release rate is related to the relative concentration of polymer in the formulation. When the polymer concentration is higher, diffusion is the major transport mechanism and the total release time is longer. This hypothesis was confirmed by a very low $k_{2} / k_{1}$ ratio and a $R / F$ below 1 for all time points in the case of for Tramadol SR, the formulation that contained a higher polymer concentration. In contrast, the lower quantity of polymer and the high quantity of soluble materials of Acetaminophen SR may have led to the formation of a more porous network during the dissolution process, resulting in a more important contribution of erosion. This was demonstrated by a superior $k_{2} / k_{1}$ ratio for Acetaminophen SR when compared to Tramadol SR. 


\subsection{Conclusions and perspectives}

This research demonstrated the potential of SD HASCA as a promising new polymer for the production of SR solid oral dosage forms. Besides safe for future use in patients, SD HASCA tablets presented excellent binding properties with no addition of a binder and drugrelease independence from the $\mathrm{CF}$ applied to produce them. The results of the clinical study confirmed the hypothesis formulated during the evaluation of SD HASCA formulations by dissolution testing, i.e., the gel formed by this polymer is able to extend the absorption of the drug after oral administration and is strong enough to resist both the peristaltic contractions and the biodegradation by $\alpha$-amylase occurring in the gastrointestinal tract. The gel formed by SD HASCA in a hydro-alcoholic medium allowed sustained drug-release and was also strong, which adds to the safety potential of SD HASCA. Dissolution testing experiments also showed that the drug-release from SD HASCA tablets was independent of both the $\mathrm{pH}$ of the SGF and the residence time in the SGF. However, variations in the residence time in the SGF slightly affected the $\alpha$-amylase-catalyzed erosion of SD HASCA formulations. The application of a two-component model that allows the investigation of the contribution of the diffusion and the erosion mechanisms to the overall drug-release rate confirmed the hypothesis that the kinetics of drug-release from SD HASCA tablets are the result of a mix of Fickian diffusion of drug molecules through the matrix and erosion of polymer chains, and that the contribution of one mechanism versus the other depends primarily on the composition of the formulation and the concentration of $\alpha$-amylase in the SIF.

However, future studies should be carried out to further characterize the polymer, evaluate its behaviour in vivo and support scale-up. Some possible prospective studies are described hereafter.

\subsubsection{Characterization of the gel layer formed by SD HASCA}

Prospective studies regarding the characterization of the gel layer formed by SD HASCA should be carried out. Examples of these studies are the evaluation of water uptake, the characterization of the moving fronts during the hydration of the matrices, the rate at 
which the gel formed by SD HASCA swells, the growth of the gel thickness in function of time and the characterization of solvent concentration gradients, i.e., solvent penetration, in SD HASCA matrices. These studies are important because the process of solvent transport into swellable polymer matrices and the corresponding dimensional changes that occur have a major influence on the profile of drug-release from these matrices. Therefore, the characterization and correlation of these phenomena is likely to allow a better predictability and an enhanced control over the process of drug-release from these matrices.

The characterization of the gel layer in a $\mathrm{pH}$ gradient as well as in a hydro-alcoholic medium should be considered.

Many approaches have been undertaken to quantify the swelling process and characterize moving fronts during the hydration of various pharmaceutical formulations. One of the methods that could be employed to characterize the gel layer is image analysis. This technique is simple and precise and permits the characterization of the changes occurring at the surface as well as inside the tablets during solvent absorption. This method has been used before to characterize swelling and solvent concentration gradients in large matrices (Moussa and Cartilier 1996; Moussa, Lenaerts et al. 1998). This technique allows the identification of several regions in the tablet, each color corresponding to the degree of hydration of a specific region. Image analysis also allows the recording of three-dimensional swelling and, therefore, the simultaneous evaluation of percentage of axial and radial swelling of the matrices (relatively to the original diameter or thickness of the tablet) in function of time, the calculation of bulk swelling using the formula of a cylinder, as well as the evolution of the gel layer thickness during hydration in function of time (Moussa and Cartilier 1996).

Nuclear magnetic resonance (NMR) imaging has also been used to investigate hydrophilic swellable polymers. For instance, the concentration changes due to solvent uptake and the evolution of the shape of the tablet at different time intervals were followed in crosslinked high-amylose starch tablets (Baille, Malveau et al. 2002). In the same way as image analysis, it allows the study of dimensional changes in both the gel layer and the core of swellable matrices (Rajabi-Siahboomi, Bowtell et al. 1994). In comparison with other techniques NMR has the advantage of being a noninvasive and nondestructive technique, as opposed to image analysis. It allows the observation of the interior of an object under 
investigation without experimental interruption, physical slicing, or other manipulations (Malveau, Baille et al. 2002). The technique can be easily used to monitor simultaneously different areas of interest in the tablet and qualitative/quantitative results can be obtained (Malveau, Baille et al. 2002). The effect of factors such as $\mathrm{pH}$ and ionic strength (Wang, Assaad et al. 2011), tablet size (Malveau, Baille et al. 2002), temperature (Therien-Aubin, Baille et al. 2005) and drug-loading on solvent uptake and tablet swelling, as well as on gel layer formation at the water/tablet interface (Therien-Aubin, Zhu et al. 2008) in the case of cross-linked high-amylose starch tablets has been evaluated using this technique.

The behaviour of the gel layer thickness in swellable matrices such as HPMC has also been evaluated using colorimetric techniques. For instance, a study used a calorimetric technique to investigate the swelling, diffusion and erosion front positions at different releasing times and the effect of drug loading on the front position and movement within the gel layer (Colombo, Bettini et al. 1999).

Assessment of water uptake by SD HASCA matrices could be carried out using gravimetric measurements. This method consists basically of weighting the tablets before incubation in a dissolution buffer, removing each tablet from the buffer at appropriate time intervals, carefully removing the excess of liquid from the surface of the tablets with filter papers, and immediate weighting. Water uptake is determined by comparison with the dry weight. New samples are weighed for every time interval.

Gravimetric measurements could be also performed for estimation of the percentage of enzymatic erosion after determined degradation periods. A method previous described for this purpose (Azevedo, Gama et al. 2003) involved the same steps described for the assessment of water uptake, except that in this case the buffer contained $\alpha$-amylase, followed by washing of the tablets with distilled water and drying in an oven, cooling until a constant weight was achieved and weighting for determination of weight loss. A control consisting of incubating the tablets in buffer alone was also used. The effect of factors such as varying the enzyme concentration, TW and the model drug on the percentage of enzyme degradation could be studied using this method.

In addition, SEM could be useful to analyze the surface morphology of SD HASCA tablets before and after enzymatic degradation. 


\subsubsection{In vivo studies and the establishment of a Level A IVIVC}

As mentioned above, further bioavailability studies in a larger number of volunteers and the establishment of a Level A IVIVC using the developed once-daily and twice-daily SD HASCA formulations as well as bioequivalence studies between these formulations and commercially available SR tramadol formulations were part of the planned experiments for this research. These clinical studies had as a goal to further substantiate the potential of SD HASCA as a new excipient for oral SR and support its industrial process scale-up.

Therefore, clinical studies using the developed SD HASCA formulations or new SD HASCA formulations with different drug candidates should be performed. These studies should involve a larger number of volunteers for a better statistical analysis of inter- and intraindividual variability. A different deconvolution approach, such as the Loo-Riegelman method (2-compartment model) or a model-independent method, could be applied to calculate the percentages of drug absorbed in function of time for each volunteer. Besides the evaluation of the bioavailability and pharmacokinetics of the formulations, the establishment of a Level A IVIVC, if possible, should be attempted. Bioequivalence studies comparing SD HASCA formulations with commercially available tramadol SR formulations could also be performed. Other possible investigation is the influence of food, and the associated delayed gastric emptying, on the drug-release characteristics of SD HASCA formulations using volunteers in the fed state, so as to ensure that the prolonged delivery of the drug is maintained, regardless of dietary status.

In the case of difficulty in achieving a point-to-point relationship between the in vitro release time course and the in vivo response time course, the subsequent development of new dissolution methods could be undertaken to help attain a Level A IVIVC. To develop an in vitro method that is directly correlated with in vivo absorption, statistically designed studies should be carried out to investigate the effects of various in vitro testing conditions on drugrelease using USP dissolution apparatuses (Qiu, Garren et al. 2003). In this research it was demonstrated that $\mathrm{pH}$ and $\alpha$-amylase (in the range of enzyme concentrations tested) do not significantly influence drug-release from SD HASCA matrices in vitro. These findings represent an advantage since it is generally easier to obtain a good IVIVC with SR dosage 
forms that are essentially unaffected by such factors. However, it is important to evaluate other variables, including agitation intensity, choice of apparatus, surfactant and ionic strength of the dissolution medium. These dissolution variables can be modified and adjusted in order to alter the dissolution profile and facilitate the establishment of a Level A IVIVC using the shape of the in vivo profile as a target for the in vitro data to match.

When dissolution rate depends on those variables, the dissolution profiles from formulations with varying in vitro release rates should be determined using a discriminatory in vitro test methodology. This dissolution methodology will better reflect the in vivo behaviour of the formulations and should be used to establish the IVIVC. In this case, a Level A IVIVC is based on the hypothesis that the same linear regression equation holds for all formulations (frequently three) with different release rates.

A validated Level A IVIVC can be used in the scale-up process of SD HASCA, for example, when changing equipment such as the spray drier used in the production of the polymer, in the optimization of SD HASCA formulations, or as a surrogate for human bioequivalence studies, which may reduce the number of bioequivalence studies performed during the initial approval process.

\subsubsection{Industrial feasibility of spray drying and the compressing methods used in this study}

\subsubsection{Spray drying}

In pharmaceutical technology, spray drying is a very important process used for obtaining dried substances with distinct properties. A typical application of spray drying in the production of excipients is spray-dried lactose, used to improve the compression properties of other powders (Sollohub and Cal 2010). Spray drying results in a product with better properties compared to other drying methods, since it produces homogenous powders (Sollohub and Cal 2010). 
The spray drier used in this research to produce SD HASCA (Büchi B-290 Mini SprayDryer, Flawill, Switzerland) is a mini device used at laboratory scale, which dries small quantities of aqueous solutions and suspensions. In order to scale-up production of SD HASCA, tests using an industrial spray drier have to be carried out. The resultant product should be analysed in order to investigate the eventual effect of changing scales on its characteristics and consequent modifications in the performance of SD HASCA as a SR excipient. For example, X-ray diffraction (XRD) can be used to characterize the crystalline or amorphous state of SD HASCA powder samples. This is important since the scale-up may alter some of the parameters employed in spray drying, such as inlet temperature and the internal moisture content of the chamber, which can affect the crystalline structure of the powder (Sollohub and Cal 2010). Scanning electron microscopy (SEM) can be used to study the morphology of the samples prepared with an industrial spray drier as well as generate information regarding the apparent particle density and porosity.

When carrying out the spray drying scale-up it should be kept in mind that the final ethanol/HASCA ration should limit the quantity of ethanol used in the process to produce the polymer as mush as possible for economical, environmental and safety reasons, while still allowing easy spray drying.

\subsubsection{Tablet compressing machines}

The 30-ton manual hydraulic press (C-30 Research \& Industrial Instruments Company, London, U.K.) used in this study is a manual press that only allows the compression of a tablet at a time, and thus it is only adequate for the production of small batches at laboratory scale for research purposes. The exact value of $\mathrm{CF}$ applied to compress the tablets using this machine is adjustable.

The single-stroke press machine (Manesty F3 Machine, Manesty Machines Ltd., Liverpool, UK) used to change the shape of the tablets is an automatic press with variable speed, which can produce 42 to 85 tablets/minute. However, as it was used in this work to produce very small batches it was hand-operated. Only one pair of punching die can be erected on this press. This machine is capable of exerting a maximum CF of 4 tons $/ \mathrm{cm}^{2}$. It has a scale 
to adjust the pressure applied to produce the tablets. The crushing strengths of the tablets must be measured and then readjusted if necessary. This press has industrial applications. However, modern rotary tablet presses holding a high number of punches can produce much larger quantities of tablets/minute and, thus, are a more adequate type of tablet presses to be used in large manufacturing of pharmaceutical products. A major study should be performed regarding the flow and compaction properties of the SD HASCA powder in order to allow the production of SD HASCA matrix tablets using industrial presses.

\subsubsection{Other possible studies}

SEM and porosimetry studies should be employed to examine the melting process observed for SA,G-2.7 in the case of SD HASCA tablets. These further studies are important since, as mention before, it was hypothesised that the increased crushing forces of the SD HASCA tablets were obtained, partly, by an intense densification of the matrices and melting under compression.

Moreover, although the results of this research showed that the influence of $\alpha$-amylase on the drug-release profiles from SD HASCA formulations was not significant, further studies using formulations with materials that can help hinder the action of the enzyme, such as maltose, a degradation product of the $\alpha$-amylase-catalyzed hydrolysis of SD HASCA, could be performed.

\subsection{References}

(US Department of Health and Human Services (2007)). "Toxicology and carcinogenesis study of glycidol (CAS No. 556-52-5) in genetically modified haploinsufficient p16(Ink4a)/p19(Arf) mice (gavage study)." Natl Toxicol Program Genet Modif Model Rep 13: 1-81.

Azevedo, H. S., F. M. Gama, et al. (2003). "In vitro assessment of the enzymatic degradation of several starch based biomaterials." Biomacromolecules 4(6): 1703-1712. 
Baille, W. E., C. Malveau, et al. (2002). "NMR imaging of high-amylose starch tablets. 1. Swelling and water uptake." Biomacromolecules 3(1): 214-218.

Brouillet, F., B. Bataille, et al. (2008). "High-amylose sodium carboxymethyl starch matrices for oral, sustained drug-release: Formulation aspects and in vitro drug-release evaluation." International Journal of Pharmaceutics 356(1-2): 52-60.

Brouillet, F., G. Baylac, et al. (2010). "High-amylose sodium carboxymethyl starch matrices for oral, sustained drug release: development of a spray-drying manufacturing process." Drug Dev Ind Pharm 36(7): 795-805.

Calinescu, C., J. Mulhbacher, et al. (2005). "Carboxymethyl high amylose starch (CM-HAS) as excipient for Escherichia coli oral formulations." European Journal of Pharmaceutics and Biopharmaceutics 60(1): 53-60.

Cartilier, L., M. Ungur, et al. (2005). Tablet Formulation for Sustained Drug-release. Canadian Patent Application 2,491,665.

Colombo, P., R. Bettini, et al. (1999). "Observation of swelling process and diffusion front position during swelling in hydroxypropyl methyl cellulose (HPMC) matrices containing a soluble drug." Journal of Controlled Release 61(1-2): 83-91.

Edge, S. and R. W. Miller (2005). Sodium starch glycolate. Handbook of pharmaceutical excipients. R. C. Rowe, P. J. Sheskey and S. C. Owen. London-Chicago, Pharmaceutical Press: 701-704.

Gallant, D. J., B. Bouchet, et al. (1992). "Physical characteristics of starch granules and susceptibility to enzymatic degradation." Eur J Clin Nutr 46(2): S3-16.

Leloup, V. M., P. Colonna, et al. (1991). "alpha-Amylase adsorption on starch crystallites." Biotechnol Bioeng 38(2): 127-134.

Leloup, V. M., P. Colonna, et al. (1992). "Physico-chemical aspects of resistant starch." Journal of Cereal Science 16(3): 253-266.

Lemieux, M., P. Gosselin, et al. (2009). "Carboxymethyl high amylose starch as excipient for controlled drug release: Mechanistic study and the influence of degree of substitution." International Journal of Pharmaceutics 382(1-2): 172-182.

Malveau, C., W. E. Baille, et al. (2002). "NMR imaging of high-amylose starch tablets. 2. Effect of tablet size." Biomacromolecules 3(6): 1249-1254.

Meyer, R. J. and A. S. Hussain (2005). FDA's ACPS Meeting, October 2005, Awareness topic: Mitigating the risks of ethanol induced dose dumping from oral sustained/controlled release dosage forms, Office of New Drugs and Office of Pharmaceutical Science, Center for Drug Evaluation and Research, FDA.

Moussa, I. S. and L. H. Cartilier (1996). "Characterization of moving fronts in cross-linked amylose matrices by image analysis." Journal of Controlled Release 42(1): 47-55.

Moussa, I. S., V. Lenaerts, et al. (1998). "Image analysis studies of water transport and dimensional changes occurring in the early stages of hydration in cross-linked amylose matrices." Journal of Controlled Release 52(1-2): 63-70.

Planchot, V., P. Colonna, et al. (1995). "Extensive degradation of native starch granules by alpha-amylase from aspergillus fumigatus." Journal of Cereal Science 21(2): 163-171.

Qiu, Y., J. Garren, et al. (2003). "Once-a-day controlled-release dosage form of divalproex sodium II: development of a predictive in vitro drug release method." Journal of Pharmaceutical Sciences 92(11): 2317-2325.

Rajabi-Siahboomi, A. R., R. W. Bowtell, et al. (1994). "Structure and behaviour in hydrophilic matrix sustained release dosage forms: 2. NMR-imaging studies of dimensional 
changes in the gel layer and core of HPMC tablets undergoing hydration." Journal of Controlled Release 31(2): 121-128.

Sollohub, K. and K. Cal (2010). "Spray drying technique: II. Current applications in pharmaceutical technology." Journal of Pharmaceutical Sciences 99(2): 587-597.

Therien-Aubin, H., W. E. Baille, et al. (2005). "Imaging of high-amylose starch tablets. 3. Initial diffusion and temperature effects." Biomacromolecules 6(6): 3367-3372.

Therien-Aubin, H., X. X. Zhu, et al. (2008). "Membrane formation and drug loading effects in high amylose starch tablets studied by NMR imaging." Biomacromolecules 9(4): 12481254.

Ungur, M., N. Yonis, et al. (2005). The evaluation of carboxymethylamylose for oral drug delivery systems: from laboratory to pilot scale. Montreal, Canada, Books of Abstracts of the ISAB2-2005 3rd International Symposium on Advanced Biomaterials/Biomechanics: 271.

Varma, M. S., A. Kaushal, et al. (2004). "Factors affecting mechanism and kinetics of drug release from matrix-based oral controlled drug delivery systems." American Journal of Drug Delivery 2(1): 43-57.

Wang, Y. J., E. Assaad, et al. (2011). "NMR imaging of chitosan and carboxymethyl starch tablets: Swelling and hydration of the polyelectrolyte complex." Int J Pharm 419(1-2): 215-221. 